\title{
Brain morphology, survival and patient-reported outcomes after cranial radiotherapy
}

Steven Nagtegaal 
Printing: Ridderprint, www.ridderprint.nl

Layout and design: Birgit Vredenburg, persoonlijkproefschrift.nl

Image cover credit: René Magritte, L'empire des lumières (1953-54) (C) 2018 C. Herscovici, London/Artists Rights Society (ARS), New York

ISBN: 978-94-64|6-99|-I

Copyright 2021 @ Steven Nagtegaal

The Netherlands. All rights reserved. No parts of this thesis may be reproduced, stored in a retrieval system or transmitted in any form or by any means without permission of the author.

Publication of this thesis was financially supported by Stichting STOPhersentumoren and the UMC Utrecht Brain Center 


\title{
Brain morphology, survival and patient-reported outcomes after cranial radiotherapy
}

\author{
Breinmorfologie, overleving en patiëntgerapporteerde \\ uitkomsten na radiotherapie voor hersentumoren \\ (met een samenvatting in het Nederlands)
}

\section{Proefschrift}

ter verkrijging van de graad van doctor aan de Universiteit Utrecht op gezag van de rector magnificus, prof.dr. H.R.B.M. Kummeling, ingevolge het besluit van het college voor promoties in het openbaar te verdedigen op donderdag 27 januari 2022 des ochtends te 10.15 uur

door

Steven Hermanus Johannes Nagtegaal

geboren op 5 februari 1993 te Nieuwegein 


\section{Promotor:}

Prof. dr. H.M. Verkooijen

\section{Copromotoren:}

Dr. J.J.C. Verhoeff

Dr. T.J. Snijders 


\section{Contents}

I. General introduction and thesis outline 7

\section{Part I: Morphological Changes in the brain after Radiotherapy}

2. Changes in cortical thickness and volume after cranial radiation treatment: a systematic review

3. Effect of radiation therapy on cerebral cortical thickness in glioma patients: treatment-induced thinning of the healthy cortex

4. Dose-dependent volume loss in subcortical deep grey matter structures after cranial radiotherapy

5. Morphological changes after cranial fractionated photon radiotherapy: localized loss of white matter and grey matter volume with increasing dose

\section{PART II: SURVIVAL AND PATIENT-REPORTED OUTCOMES IN BRAIN METASTASES}

6. Comparing survival predicted by the diagnosis-specific Graded Prognostic 103 Assessment (DS-GPA) to actual survival in patients with $1-10$ brain metastases treated with stereotactic radiosurgery

7. Age, pathology and CA-125 are prognostic factors for survival in patients with brain metastases from gynaecological tumours

8. Socioeconomic status is not associated with survival in patients with brain metastases treated with stereotactic radiotherapy

9. Clinical outcomes, imaging and quality of life in patients with brain 155 metastases treated with radiotherapy: design and first results of the COIMBRA prospective cohort

10. General discussion

APPENDICES

Dutch summary

Dankwoord

List of publications

Curriculum Vitae Auctoris 


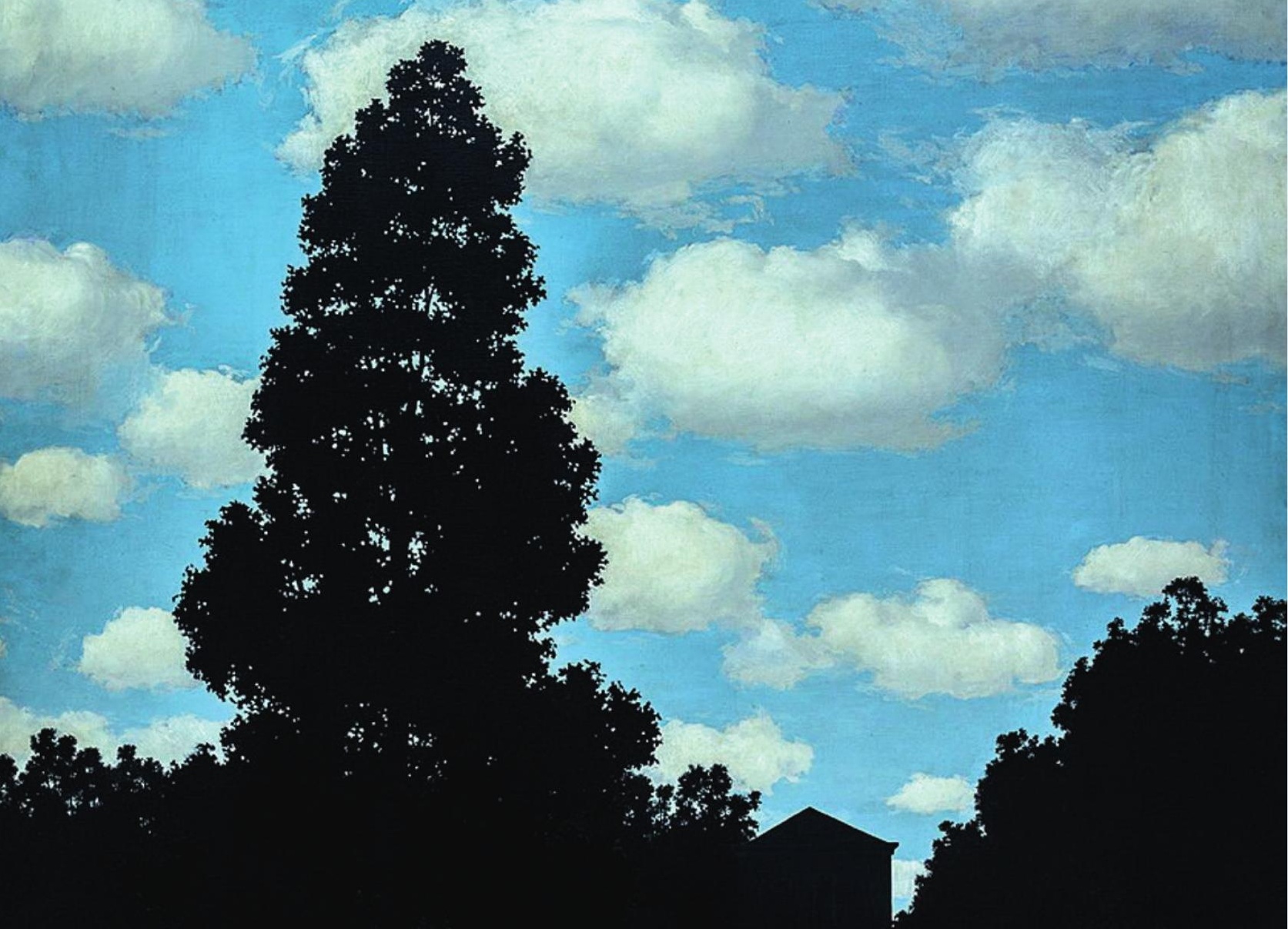

4t

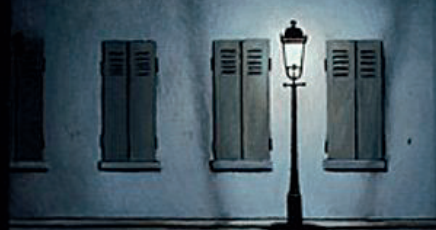




\section{Chapter I}

General introduction and thesis outline 
Malignant brain tumours can be divided into primary and secondary tumours. Primary tumours originate in the brain, being neoplastic growths from brain cells. Secondary brain tumours on the other hand have primary origins elsewhere in the body. They metastasize to the brain, and are therefore called brain metastases. Both primary and secondary tumours are commonly treated with external beam radiotherapy (EBRT, or simply RT), a noninvasive technique in which ionizing radiation is delivered to the location of the tumour. The aim is to damage the DNA within the neoplastic cells, causing disturbance of the mitotic cycle and ultimately leading to cell death. It is often given in fractions, meaning that the dose is delivered is separate treatment sessions over the course of several days or weeks. This allows the healthy tissue surrounding the tumour to repair radiation damage, while irreparably destructing tumour cells. Currently, malignant brain tumours are incurable. With increasing effectiveness of novel therapies, the focus of both medical care and research shift towards improving patients' quality of life. This includes an appreciation of the long-term adverse effects of treatment, which were not recognized in times when survival was limited.

This thesis focuses on radiotherapy for both primary brain tumours and brain metastases, and attempts to answer questions in relation to improving quality of life for both types. For primary brain tumours, I describe to what extent morphological changes occur after radiotherapy, with the hypothesis that this is the cause for cognitive decline commonly seen in long-term survivors of brain tumours. For brain metastases, I discuss the survival after radiation treatment, and whether this can be estimated with baseline factors. Additionally, I present the design and preliminary results of a cohort study of patients that undergo radiotherapy for brain metastases. The aim of this study is to gather information on quality of life, and to facilitate future inclusion in randomized clinical trials.

\section{PART I: MORPHOLOGICAL CHANGES IN THE BRAIN AFTER RADIOTHERAPY}

\section{Glioma epidemiology and treatment}

In the Netherlands, I,300 patients are diagnosed with primary brain tumours annually.' Of them, the vast majority originate from glial cells, and are therefore called glioma. Historically, these were classified into four grades based on histopathological characteristics, according to guidelines from the World Health Organization (WHO) published in 2007.2 Gliomas of WHO grade I and II, which include pilocytic and diffuse astrocytoma and oligodendroglioma, are also referred to as "low-grade". Grades III (anaplastic astrocytoma and anaplastic oligodendroglioma) and IV (glioblastoma) in turn were named high-grade. In an updated grading system published in 2016, molecular markers were added to account for recent insights into the effects of oncogenetics on the carcinogenesis of glioma. ${ }^{3}$ Mutations in isocitrate dehydrogenase (IDH), classified as IDH-mutant or IDH-wildtype, have been 
added to the grading system, as has the presence or absence of Ip/19q codeletion. Next to differences in tumour origin and growth, these markers also have prognostic consequences, adding to the clinical relevance of histopathological classification. The estimated median survival after diagnosis ranges from 8 - 15 years for IDH-mutant grade II and III astrocytoma and low-grade oligodendroglioma; it is lower for high-grade oligodendroglioma with 5-9 years. $^{4-8}$ IDH-wildtype diffuse and anaplastic astrocytoma have a prognosis of 1.5 to 3 years. Finally, glioblastoma has the poorest prognosis, with a median observed survival of 10-12 months. 910

Currently, treatment options for glioma are surgery, radiotherapy and chemotherapy, typically given in combination. The exact treatment regimen is dependent on various factors, including tumour grade, location, resectability, size, desirability of tissue for histopathological examination, and the patient's condition and preference." In the case of low-grade glioma, one option is "watchful waiting", also called "wait and scan": withholding treatment in favour of regular radiological follow-up. When treatment in decided on, resection is followed by radiotherapy (50.4 Gy in 28 fractions) and chemotherapy. The latter is composed of either a combination of procarbazine, lomustine, and vincristine (PCV) in case of a Ip/I9q-codeletion, or temozolomide when no codeletion is present. Patients with Grade III gliomas are always advised to undergo maximum safe resection, followed by radiotherapy (59.4 Gy in 33 fractions) with or without PCV, again dependent on Ip/19q codeletion. For those without a codeletion, but with an IDH-mutation, temozolomide is advised. ${ }^{12}$ Since the landmark publication by Stupp et al., the standard treatment for glioblastoma is neurosurgical excision followed by radiotherapy (60 Gy in 30 fractions) with concomitant and adjuvant chemotherapy with temozolomide. ${ }^{13}$ For frail, mostly elderly, patients, a less intensive treatment of either chemotherapy or short-course radiation is often applied..$^{14}$

\section{Radiotherapy and morphological changes}

As mentioned above, the aim of radiotherapy is to cause damage in the DNA of tumour cells but not in the surrounding tissue. This means a delicate balance exists in which the tumour needs to be adequately treated to improve survival, while limiting the amount of damaging radiation to healthy tissues. Several factors dictate whether this balance can be achieved. One is the limits of the radiotherapy technique and physics themselves. Especially in the magnitudes used to treat gliomas, it is impossible for radiotherapy dose to change from high to no dose at the edge of the tumour; there is always a gradient outside the tumour. Therefore, maintaining a high dose within the desired area means a significant amount of radiation must be received by the surrounding tissue. Secondly, safety margins are used to guarantee adequate tumour coverage. In radiotherapy for glioma, several treatment volumes are defined to determine the total volume to be irradiated (Figure I). ${ }^{15}$ Firstly, the gross tumour volume (GTV) is, as the name implies, the total volume of 
the neoplasm as seen on imaging. An extra margin is added on top of the GTV to account for microscopic spread of the tumour not visible on imaging. In the local guidelines of the UMC Utrecht, this margin is $1 \mathrm{~cm}$ for low-grade and $2 \mathrm{~cm}$ for high-grade glioma. The total volume of GTV plus margin is called the clinical target volume (CTV), and is the volume that needs to be treated in order to achieve the desired therapeutic effect. A final margin is added to account for uncertainties in target planning and dose delivery and to make sure the CTV is properly irradiated. This final volume is called the planning treatment volume (PTV), and in our department is created by adding a margin of $0.2 \mathrm{~cm}$ to the PTV. All these extra margins can result in substantial increase in total irradiated volume, especially in large tumours. This means that more healthy brain tissue receives radiation, and the edges of the gradient between high and low dose shifts outward.

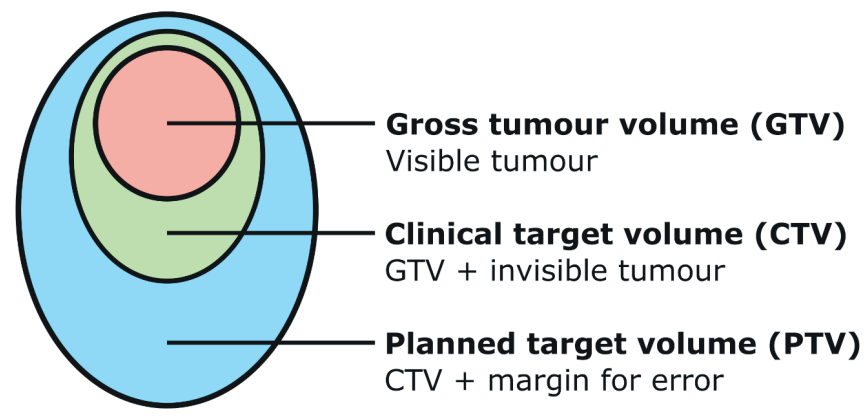

Figure I Treatment volumes in radiotherapy

This dose in healthy tissue can lead to adverse effects. Cognitive impairments are a common phenomenon after cranial radiotherapy. It is estimated that $50-90 \%$ of patients receiving radiotherapy to the brain experience progressive cognitive disability. ${ }^{16,17}$ The severity varies from mild impairment to symptoms similar to progressive dementia. The exact aetiology of this disabling consequence of tumour treatment remains unknown, but there are multiple hypotheses, including neuronal dysfunction, vascular damage and disturbed neurogenesis. The true cause is most likely multifactorial. ${ }^{16-18}$ A challenge in discovering the cause of cognitive decline after treatment is the fact that the tumour itself is can also lead to cognitive symptoms. ${ }^{19}$

In order to elucidate the origin of radiation-induced cognitive decline, brain imaging has been examined to see if changes in brain anatomy (or morphology) are observed after radiotherapy. Different parts of the brain have been examined (Figure 2), which I will discuss below. 


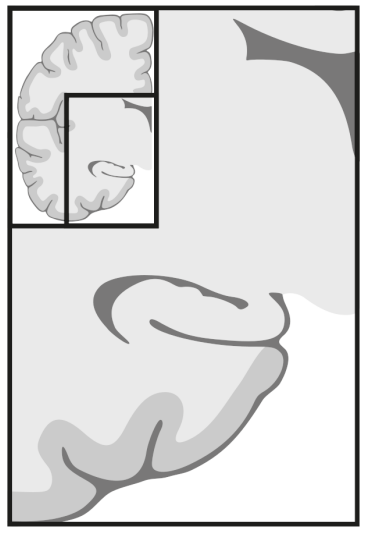

Before

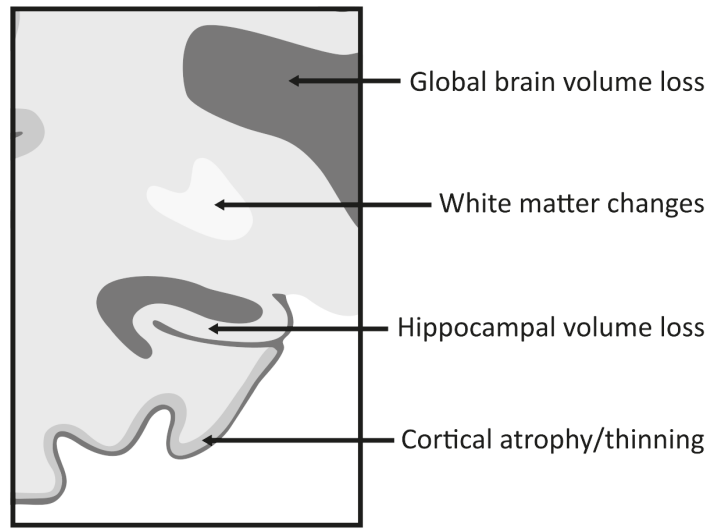

After

Figure 2 Changes in the brain after radiotherapy, as seen on MRI. Image from Nagtegaal et al. ${ }^{20}$

\section{Cortex}

The cerebral cortex contains a vast number of neurons, and has a thickness between I and $4.5 \mathrm{~mm}$ in vivo, depending on location. ${ }^{21}$ The neurons are interconnected in an immensely complex network and form the tissue which is essential for the execution of all cognitive processes. ${ }^{22}$ It is comprised of both neuronal cell bodies and fibres, together with glial cells and blood vessels. ${ }^{23}$ Both damage to neurons and vasculature can lead to disruption in the cortical network, and have been linked to changes in cognitive abilities. ${ }^{17,24}$ Before the era of digital image analysis, macroscopic cortical atrophy as seen on routine CT or MRI had already been associated with radiotherapy and cognitive function. ${ }^{25-28}$ Although decrease in cortical thickness is part of the normal aging process, ${ }^{29}$ accelerated decrease has been linked with several diseases, including Alzheimer's disease ${ }^{30,31}$, Parkinson's disease ${ }^{32}$ and depression. ${ }^{33,34}$ Furthermore, it has also been associated with cognitive impairments, ${ }^{35,36}$ which leads to the hypothesis that cortical thinning is a contributing factor to the cognitive impairments seen after radiotherapy. Several studies have already been performed on the effect of radiotherapy on cortical thickness. Karunamuni et al. was the first to show dose-dependent cortical thinning after RT in 15 glioma patients. ${ }^{37}$ Seibert et al. went on to examine brain regions associated with cognitive function, and found these to be susceptible to radiation-induced damage. ${ }^{38}$ However, these studies examined the brain per lobe or cortical region, which may overlook smaller areas of susceptibility.

\section{Deep grey matter}

Two grey matter structures, the hippocampus and the amygdala, show susceptibility to radiation damage, again with greater volume loss with increasing dose..$^{39,40}$ Furthermore, the dose to the hippocampus has been shown to negatively affect neurocognitive outcome after RT. ${ }^{41}$ 
Less is known about the susceptibility to radiation damage of other subcortical grey matter structures, such as the nucleus accumbens, caudate nucleus, globus pallidus, putamen, and thalamus. These structures are vital links in complex networks involved in a variety of cognitive functions. For example, the amygdala is part of a system for emotional control, and the hippocampus contributes to normal memory functions. ${ }^{42}$ And like the cerebral cortex, atrophy of these deep GM structures is associated with impaired cognitive function in patients with degenerative brain diseases as well as healthy ageing. ${ }^{43-45}$ This relation is most pronounced in Alzheimer's disease, with the volume of all mentioned structures, with the exception of globus pallidus, being associated with cognitive impairment. ${ }^{46-48}$ Globus pallidus volume in its turn is associated with cognitive outcomes in Huntington's disease and age-related cognitive impairments. ${ }^{49,50}$

\section{White matter}

Since the advent of MR imaging, necrosis has been observed in the post-RT white matter. ${ }^{51}$ This has been further studied after the development of diffusion tensor imaging (DTI), which is the most sensitive technique to visualize white matter tracts, and detect white matter damage. When this imaging modality was used to study the effects of radiation on the brain, otherwise normal-appearing white matter showed signs of demyelination, structural degradation of axonal fibres, and changes in diffusivity parameters. ${ }^{52,53}$ The observed damage increased with higher applied dose, suggesting a relation to the administered radiotherapy.

\section{New organs at risk?}

Because of known radiation damage, several structures have been branded as "organs at risk", each with their own dose limits. These include the brain stem, optic chiasm, cochlea, and pituitary gland. ${ }^{54}$ Should a relation be found between dose-related damage in healthy grey or white matter and cognitive decline, these brain areas could be added to this list. In order to do that, however, the exact relation between radiotherapy and morphometric changes in healthy brain tissue needs to be examined first. Luckily, several image processing techniques have been developed to automatically segment and analyse changes in grey and white matter volume and cortical thickness. Originally these tools were developed to study changes related to healthy aging and degenerative brain disease. Applying them to a group of patients irradiated for glioma forms the first step toward the identification of areas that are susceptible to radiation-induced dose-dependent damage leading to cognitive impairments. In part I of this thesis, I describe four studies on this subject. 


\section{PART II: SURVIVAL AND PATIENT-REPORTED OUTCOMES IN BRAIN METASTASES}

\section{Epidemiology and treatment}

Brain metastases occur in $20-40 \%$ of all cancer patients, with $8-10 \%$ having intracranial metastasis at initial presentation. ${ }^{55-59}$ Every year, around 7,000 patients develop brain metastases in the Netherlands. ${ }^{60,61}$ This incidence is expected to increase with more effective treatments for the primary tumours, thereby improving survival and thus increasing the time for possible dissemination of tumour to the brain. ${ }^{57,62}$ Brain metastases most frequently originate from lung cancer, breast cancer and melanoma, but in up to $14 \%$ of patients, the metastases are of unknown origin. ${ }^{57,62-65}$

As with primary brain tumours, treatment for brain tumours can consist of surgery, radiotherapy and systemic therapy. The final decision should always be made after consultation in a multidisciplinary tumour board, and available treatment options should also be discussed with the patient. Although every patient is different, general guidelines exist to facilitate clinical decision making. ${ }^{66}$

Radiotherapy is an important option for all patients opting to undergo treatment for brain metastases. In general, two options exist for radiotherapy of brain metastases: stereotactic radiosurgery (SRS) and whole-brain radiotherapy (WBRT) (Figure 3). SRS is a viable option when the number of lesions is limited to 10 , with a total volume below $30 \mathrm{~cm}^{3}{ }^{3}$. When using a thermoplastic immobilization mask, only I mm is added to the CTV, the latter being equal to the GTV. The radiation is delivered in a single fraction, with a dose usually between 18 and $24 \mathrm{~Gy}$, dependent on the size of the lesion. WBRT is advised when SRS is not indicated, but a wish for treatment exists. As the name implies, it is delivered to the entire brain, in either 5 fractions of 4 Gy or in 10 fractions of 3 Gy. Before the advent of SRS, WBRT was the standard radiotherapeutic treatment for brain metastases; currently SRS has become a common treatment in patient with a limited number of lesions. ${ }^{68}$ 


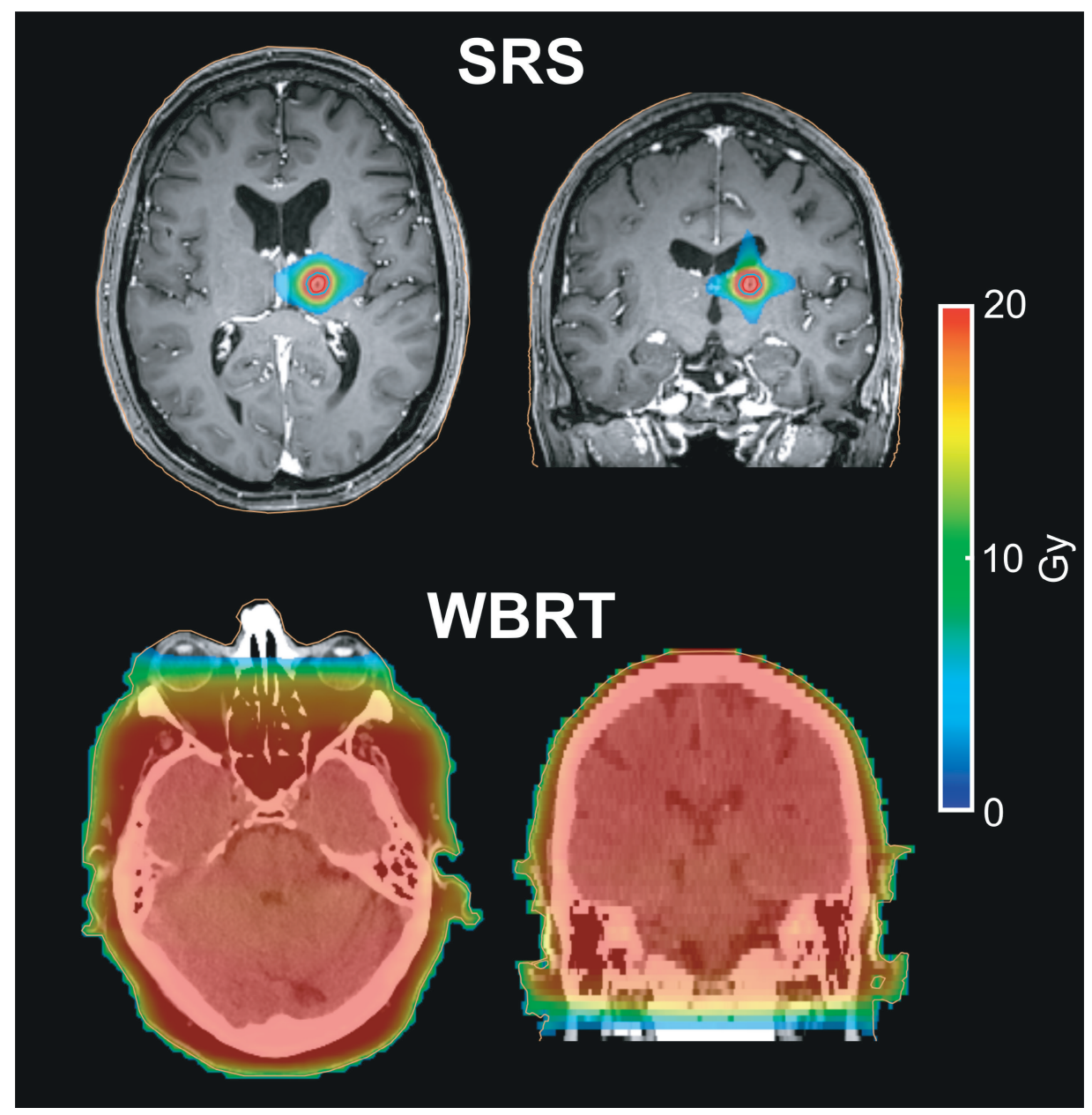

Figure 3 Dose distributions in stereotactic radiosurgery (SRS), and whole-brain radiotherapy (WBRT) as seen on planning MRI.

Patients can be considered for surgical excision if they have a solitary metastasis larger than $2.5 \mathrm{~cm}$ in diameter, and have a favourable prognosis. Local radiotherapy after surgery, even after complete resection, increases local control and decreases the risk of local recurrence; ${ }^{69}$ whole-brain radiotherapy after resection is not associated with clinical benefit. ${ }^{70}$

Another option that is usually considered is best supportive care. Especially when the expected survival is limited, the desirability of continuing tumour-directed treatment should be discussed with the patient. To facilitate this process, several scores have been developed to predict survival of patients with brain metastases. 


\section{Prognostic scores for brain metastases}

The most widely used score is the Recursive Partitioning Analysis (RPA), first published by Gaspar et al. in 1997 7l. It uses a patient's Karnofsky Performance Score (KPS), age, presence of extracranial metastases, and control of the primary tumour to assign them one of three grades, each with an estimated median survival. The most favourable, grade I, is given to patients with a KPS $\geq 70$, age $<65$, controlled primary tumour and no other systemic metastases. Patients not satisfying those criteria are determined as being grade II if they have an KPS $\geq 70$. The remaining patients are grade III.

The RPA, however, has its limitations. Firstly, the data used to create the RPA were taken from patients enrolled in clinical trials, meaning the generalizability to all patients with brain metastases is limited. Secondly, the criterion of "controlled primary" is ill-defined, leading to possible inconsistencies between physicians and patients. Finally, the number of brain metastases had proven to be of prognostic value, but was not incorporated in the RPA. ${ }^{55}$

To account for this the Graded Prognostic Assessment (GPA) was created by Sperduto et al. in 2008. ${ }^{72}$ In this model, a score of 0.5 or I is assigned for certain values of each prognostic factor: age, KPS, number of brain metastases and presence of extracranial metastases. Later, separate models were made for the five most common primary tumours: lung cancer, breast cancer, melanoma, renal cell carcinoma and gastro-intestinal cancer. ${ }^{73}$ This disease-specific GPA (DS-GPA) features separate scoring methods for each primary tumour. For each total score an estimated median survival is given, which can be used to inform physicians and patients on the expected prognosis. The predictive models are constantly being updated with the newest findings, using large samples of multi-institutional clinical patient data. The scores for lung cancer and melanoma now feature the molecular subtypes of the tumour, and the model for RCC has been updated to include haemoglobin count $(\mathrm{Hb})$ at baseline. ${ }^{74-76}$ The most recent models are freely available on brainmetgpa.com. ${ }^{77}$

\section{Challenges in the enrolment of patients with brain metastases in ran- domized trials}

New treatments are continuously being developed for brain metastases, all of which warrant multiple trials to assess safety, determine the efficacy, and optimize protocols. The gold standard of clinical trials is the randomised controlled trial (RCT). However, RCTs assessing novel oncological treatments are faced with recurrent problems, such as failure to reach target inclusion, highly selective study populations and low generalizability of results. To help address these issues, the concept of trials within cohorts (TwiCs) was developed. ${ }^{78,79}$ 


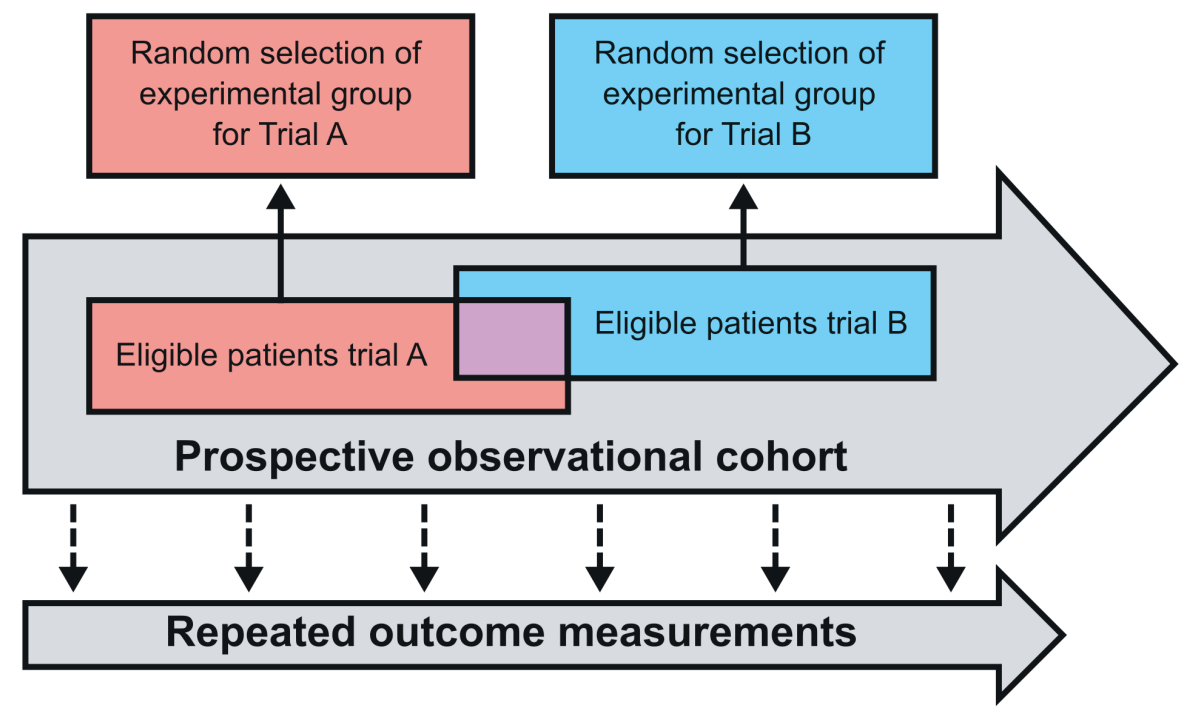

Figure 4 Graphical overview of the TwiCs design. Participants' clinical and self-reported outcomes are regularly measured. For each randomized trial a sub-cohort of eligible patient is identified. Patients from the sub-cohort are randomly selected to undergo the intervention, and their outcomes are then compared to the other patients in the sub-cohort who undergo standard care. This process repeats for each new TwiC that is conducted; patients may be eligible for multiple trials. Adapted from Relton et al. ${ }^{78}$

The basis of the TwiCs approach (also known as the cohort multiple Randomised Controlled Trial, cmRCT) is creating an observational prospective cohort, consisting of patients with the same condition of interest undergoing standard treatment. The patient characteristics are gathered at baseline, and clinical and patient-reported outcomes (PROs) are collected at fixed time points after inclusion. When a new intervention is ready for formal evaluation, eligible patients within the observational cohort are identified (Figure 4). From this socalled sub-cohort, patients will be randomly selected, and asked for consent to undergo the new intervention. The other eligible patients will not be approached and undergo the standard treatment. Outcomes of the selected patients receiving the new intervention will be compared to the outcomes of the non-selected patients receiving the standard treatment. Within this cohort, the same process can be simultaneously performed for other interventions. Advantages of the TwiCs design include on-going information on the natural history of the condition and standard treatment, the ability to facilitate multiple simultaneous randomised evaluations, the improved comparability between trials and the patient-centred informed consent procedure. ${ }^{78,79}$ 


\section{THESIS OUTLINE}

\section{Part I}

The first part of this thesis will address the effect of radiotherapy on the morphology of healthy brain tissue. The main goal is to assess whether the volume or thickness of different tissue types and brain regions changes after radiotherapy, and whether the rate of morphological change is correlated with the administered dose.

Chapter 2 summarizes the available literature on the effect of radiotherapy on the thickness and volume of the cerebral cortex.

Chapter 3 examines the effect of radiotherapy on cortical thickness in glioma patients, and assesses whether the rate of thickness change is related to the applied dose.

Chapter 4 evaluates the changes in volume of subcortical deep grey matter structures after radiotherapy, and correlates this to the administered dose.

Chapter 5 uses deformation-based and vortex-based morphometry to measure the impact of radiotherapy dose on volumetric changes in the entire brain, as well as total volumes of grey matter, white matter and cerebrospinal fluid.

\section{Part II}

The second part of this thesis focusses on prognostic factors for survival in brain metastases, and describes the COIMBRA cohort. The main goals are to identify ways to predict survival for patients with brain metastases, and to explain how the COIMBRA cohort contributes to the future of brain metastasis research.

Chapter 6 evaluates the performance of the DS-GPA prognostic model in patients with brain metastases, and compares the predicted survival to the actual survival.

Chapter 7 identifies prognostic factors for survival in patients with brain metastases originating from gynaecological tumours.

Chapter 8 assesses the impact of the socioeconomic status on survival in patients with brain metastases, corrected for the relevant clinical factors.

Chapter 9 describes the protocol of the COIMRBA cohort, and summarizes the preliminary findings at baseline. 


\section{REFERENCES}

I. Netherlands Comprehensive Cancer Organization. Netherlands Cancer Registry. Www. cijfersoverkanker.nl. Published 2020.

2. Louis DN, Ohgaki H, Wiestler OD, et al. The 2007 WHO Classification of Tumours of the Central Nervous System. Acta Neuropathol. 2007;1 I4(2):97-109. doi:I0.1007/s00401-007-0243-4

3. Louis DN, Perry A, Reifenberger G, et al. The 2016 World Health Organization Classification of Tumors of the Central Nervous System: a summary. Acta Neuropathol. 2016;131(6):803-820. doi:10.1007/s00401-016-1545-I

4. Eckel-Passow JE, Lachance DH, Molinaro AM, et al. Glioma Groups Based on Ip/I9q, IDH, and TERT Promoter Mutations in Tumors. N Engl J Med. 20I5;372(26):2499-2508. doi:I0.1056/NEJMoal407279

5. Hartmann C, Hentschel B, Wick W, et al. Patients with IDHI wild type anaplastic astrocytomas exhibit worse prognosis than IDHI-mutated glioblastomas, and IDHI mutation status accounts for the unfavorable prognostic effect of higher age: implications for classification of gliomas. Acta Neuropathol. 20I0;120(6):707-7I8. doi:I0.1007/s0040I-0I0-078I-z

6. van den Bent MJ, Weller M, Wen PY, Kros JM, Aldape K, Chang S. A clinical perspective on the 2016 WHO brain tumor classification and routine molecular diagnostics. Neuro Oncol. 2017;19(5):614-624. doi:10.1093/neuonc/now277

7. Ohgaki H, Kleihues P. Population-Based Studies on Incidence, Survival Rates, and Genetic Alterations in Astrocytic and Oligodendroglial Gliomas. J Neuropathol Exp Neurol. 2005;64(6):479-489. doi: I0.1093/jnen/64.6.479

8. Lassman AB, Iwamoto FM, Cloughesy TF, et al. International retrospective study of over 1000 adults with anaplastic oligodendroglial tumors. Neuro Oncol. 20 II;13(6):649-659. doi:I0.1093/neuonc/nor040

9. Ostrom QT, Gittleman H, Xu J, et al. CBTRUS Statistical Report: Primary Brain and Other Central Nervous System Tumors Diagnosed in the United States in 2009-2013. Neuro Oncol. 2016;18(suppl_5):vl-v75.doi:I0.1093/neuonc/now207

10. Korja M, Raj R, Seppä K, et al. Glioblastoma survival is improving despite increasing incidence rates: a nationwide study between 2000 and 2013 in Finland. Neuro Oncol. 2019;21 (3):370-379. doi:10.1093/ neuonc/noyl64

II. Landelijke werkgroep neuro-oncologie. Richtlijn gliomen, versie 4.0. Federatie Medisch Specialisten. https://richtlijnendatabase.nl/richtlijn/gliomen/gliomen_-_startpagina.html. Published 2019.

12. van den Bent MJ, Baumert B, Erridge SC, et al. Interim results from the CATNON trial (EORTC study 26053-22054) of treatment with concurrent and adjuvant temozolomide for Ip/I9q non-co-deleted anaplastic glioma: a phase 3, randomised, open-label intergroup study. Lancet. 2017;390(I0103):16451653. doi:10.1016/S0|40-6736(17)31442-3

13. Stupp R, Hegi ME, Mason WP, et al. Effects of radiotherapy with concomitant and adjuvant temozolomide versus radiotherapy alone on survival in glioblastoma in a randomised phase III study: 5-year analysis of the EORTC-NCIC trial. Lancet Oncol. 2009;10(5):459-466. doi:10.1016/ SI470-2045(09)70025-7

14. Wick W, Platten M, Meisner C, et al. Temozolomide chemotherapy alone versus radiotherapy alone for malignant astrocytoma in the elderly: the NOA-08 randomised, phase 3 trial. Lancet Oncol. 2012;13(7):707-7I5. doi:10.1016/SI470-2045(I2)70164-X

15. Burnet NG. Defining the tumour and target volumes for radiotherapy. Cancer Imaging. 2004;4(2): 153|6I. doi:I0.1 I02//470-7330.2004.0054

16. Makale MT, McDonald CR, Hattangadi-Gluth JA, Kesari S. Mechanisms of radiotherapy-associated cognitive disability in patients with brain tumours. Nat Rev Neurol. 2017;13(1):52-64. doi:10.1038/ nrneurol.2016.185

17. Greene-Schloesser D, Robbins ME. Radiation-induced cognitive impairment-from bench to bedside. Neuro Oncol. 2012;14(SUPPL.4):iv37-iv44. doi:10.1093/neuonc/nos196

18. Lee YW, Cho HJ, Lee WH, Sonntag WE. Whole brain radiation-induced cognitive impairment: pathophysiological mechanisms and therapeutic targets. Biomol Ther (Seoul). 2012;20(4):357-370. doi:10.4062/biomolther.2012.20.4.357 
19. van Kessel E, Baumfalk AE, van Zandvoort MJE, Robe PA, Snijders TJ. Tumor-related neurocognitive dysfunction in patients with diffuse glioma: a systematic review of neurocognitive functioning prior to anti-tumor treatment. J Neurooncol. 2017;134(I):9-18. doi:10.1007/s II060-017-2503-z

20. Nagtegaal SHJ, David S, Snijders TJ, Philippens MEP, Leemans A, Verhoeff JJC. Effect of radiation therapy on cerebral cortical thickness in glioma patients: treatment-induced thinning of the healthy cortex. Neuro-Oncology Adv. 2020;2(I). doi:I0.1093/noajnl/vdaa060

21. Fischl B, Dale AM. Measuring the thickness of the human cerebral cortex from magnetic resonance images. Proc Natl Acad Sci U S A. 2000;97(20):I 1050-1 I055. doi:10.1073/pnas.200033797

22. Kandel ER, Schwartz JH, eds. Principles of Neural Science, Fifth Edition. New York: McGraw-Hill; 2013.

23. Mancall EL, Brock DG, eds. Gray's Clinical Neuroanatomy. Philadelphia: Elsevier Saunders; 2010.

24. Bosma I, Douw L, Bartolomei F, et al. Synchronized brain activity and neurocognitive function in patients with low-grade glioma: a magnetoencephalography study. Neuro Oncol. 2008;10(5):734-744. doi:10.1215/15228517-2008-034

25. Valk PE, Dillon WP. Radiation injury of the brain. AJNR Am J Neuroradiol. 1991;12(I):45-62. http://www. ncbi.nlm.nih.gov/pubmed/7502957.

26. Postma TJ, Klein M, Verstappen CCP, et al. Radiotherapy-induced cerebral abnormalities in patients with low-grade glioma. Neurology. 2002;59(I):I21-123.

27. Rabin BM, Meyer JR, Berlin JW, et al. Radiation-induced changes in the central nervous system and head and neck. Radiographics. 1996; I6(5): I055-1072. doi:I0.1/48/radiographics. 16.5.8888390

28. Harder $\mathrm{H}$, Holtel $\mathrm{H}$, Bromberg JEC, et al. Cognitive status and quality of life after treatment for primary CNS lymphoma. Neurology. 2004;62(4):544-547. http://www.embase.com/search/ results?subaction=viewrecord\&from=export\&id=L38252793.

29. Salat DH. Thinning of the Cerebral Cortex in Aging. Cereb Cortex. 2004;14(7):721-730. doi:10.1093/ cercor/bhh032

30. Du A-T, Schuff N, Kramer JH, et al. Different regional patterns of cortical thinning in Alzheimer's disease and frontotemporal dementia. Brain. 2007;130(4):I 159-I 166. doi:10.1093/brain/awm0I6

31. Yin C, Li S, Zhao W, Feng J. Brain imaging of mild cognitive impairment and Alzheimer's disease. Neural Regen Res. 2013;8(5):435-444. doi:10.3969/j.issn.1673-5374.2013.05.007

32. Pereira JB, Ibarretxe-Bilbao N, Marti M-J, et al. Assessment of cortical degeneration in patients with Parkinson's disease by voxel-based morphometry, cortical folding, and cortical thickness. Hum Brain Mapp. 2012;33(II):252I-2534. doi:I0.1002/hbm.21378

33. Lim HK, Jung WS, Ahn KJ, et al. Regional cortical thickness and subcortical volume changes are associated with cognitive impairments in the drug-naive patients with late-onset depression. Neuropsychopharmacology. 2012;37(3):838-849. doi:10.1038/npp.2011.264

34. Peterson BS, Warner $\mathrm{V}$, Bansal R, et al. Cortical thinning in persons at increased familial risk for major depression. Proc Natl Acad Sci. 2009;106(15):6273-6278. doi:I0.1073/pnas.0805311106

35. Seo SW, Im K, Lee J-M, et al. Cortical thickness in single- versus multiple-domain amnestic mild cognitive impairment. Neuroimage. 2007;36(2):289-297. doi:10.1016/j.neuroimage.2007.02.042

36. Garcia-Diaz Al, Segura B, Baggio HC, et al. Cortical thinning correlates of changes in visuospatial and visuoperceptual performance in Parkinson's disease: A 4-year follow-up. Parkinsonism Relat Disord. 2018;46:62-68. doi:10.1016/j.parkreldis.2017.11.003

37. Karunamuni R, Bartsch H, White NS, et al. Dose-Dependent Cortical Thinning After Partial Brain Irradiation in High-Grade Glioma. Int J Radiat Oncol Biol Phys. 2016;94(2):297-304. doi:I0.1016/j. ijrobp.2015.10.026

38. Seibert TM, Karunamuni R, Kaifi S, et al. Cerebral Cortex Regions Selectively Vulnerable to Radiation Dose-Dependent Atrophy. Int J Radiat Oncol Biol Phys. 2017;97(5):910-918. doi:10.1016/j. ijrobp.2017.01.005

39. Huynh-Le M-P, Karunamuni R, Moiseenko V, et al. Dose-dependent atrophy of the amygdala after radiotherapy. Radiother Oncol. 2019;136:44-49. doi:I0.1016/j.radonc.2019.03.024

40. Seibert TM, Karunamuni R, Bartsch H, et al. Radiation Dose-Dependent Hippocampal Atrophy Detected With Longitudinal Volumetric Magnetic Resonance Imaging. Int J Radiat Oncol. 2017;97(2):263-269. doi:10.1016/j.ijrobp.2016.10.035 
41. Gondi V, Hermann BP, Mehta MP, Tomé WA, Tome WA. Hippocampal dosimetry predicts neurocognitive function impairment after fractionated stereotactic radiotherapy for benign or low-grade adult brain tumors. Int J Radiat Oncol Biol Phys. 2013;83(4):e487-93. doi:10.1016/j. ijrobp.2011.10.021

42. Vanderah T, Gould D. Nolte's the Human Brain: An Introduction to Its Functional Anatomy. 7th ed. Philadelphia, PA: Elsevier; 2015.

43. Pagnozzi AM, Fripp J, Rose SE. Quantifying deep grey matter atrophy using automated segmentation approaches: A systematic review of structural MRI studies. Neuroimage. 2019;201:1/6018. doi:10.1016/j. neuroimage.2019.116018

44. Hughes EJ, Bond J, Svrckova P, et al. Regional changes in thalamic shape and volume with increasing age. Neuroimage. 2012;63(3):1 134-1|42. doi:10.1016/j.neuroimage.2012.07.043

45. Zanchi D, Giannakopoulos P, Borgwardt S, Rodriguez C, Haller S. Hippocampal and Amygdala Gray Matter Loss in Elderly Controls with Subtle Cognitive Decline. Front Aging Neurosci. 2017;9. doi:I0.3389/fnagi.2017.00050

46. de Jong LW, van der Hiele K, Veer IM, et al. Strongly reduced volumes of putamen and thalamus in Alzheimer's disease: an MRI study. Brain. 2008;13I(Pt 12):3277-3285. doi:10.1093/brain/awn278

47. Nie X, Sun Y, Wan S, et al. Subregional Structural Alterations in Hippocampus and Nucleus Accumbens Correlate with the Clinical Impairment in Patients with Alzheimer's Disease Clinical Spectrum: Parallel Combining Volume and Vertex-Based Approach. Front Neurol. 2017;8:399. doi:I0.3389/fneur.2017.00399

48. Yi H-A, Möller C, Dieleman N, et al. Relation between subcortical grey matter atrophy and conversion from mild cognitive impairment to Alzheimer's disease. J Neurol Neurosurg Psychiatry. 2016;87(4):425432. doi:10.1136/jnnp-2014-309105

49. Aylward EH, Harrington DL, Mills JA, et al. Regional atrophy associated with cognitive and motor function in prodromal Huntington disease. J Huntingtons Dis. 2013;2(4):477-489. doi:I0.3233/JHD-I30076

50. Valdés Hernández MC, Clark R, Wang S-H, et al. The striatum, the hippocampus, and short-term memory binding: Volumetric analysis of the subcortical grey matter's role in mild cognitive impairment. Neurolmage Clin. 2020;25:102158. doi:10.1016/j.nicl.2019.102158

51. Kumar AJ, Leeds NE, Fuller GN, et al. Malignant Gliomas: MR Imaging Spectrum of Radiation Therapyand Chemotherapy-induced Necrosis of the Brain after Treatment. Radiology. 2000;217(2):377-384. doi:10.1 148/radiology.217.2.r00nv36377

52. Nagesh V, Tsien Cl, Chenevert TL, et al. Radiation-Induced Changes in Normal-Appearing White Matter in Patients With Cerebral Tumors: A Diffusion Tensor Imaging Study. Int J Radiat Oncol. 2008;70(4):1002-1010. doi:10.1016/j.ijrobp.2007.08.020

53. Zhu T, Chapman CH, Tsien C, et al. Effect of the Maximum Dose on White Matter Fiber Bundles Using Longitudinal Diffusion Tensor Imaging. Int J Radiat Oncol. 2016;96(3):696-705. doi:I0.1016/j. ijrobp.2016.07.010

54. Scoccianti S, Detti B, Gadda D, et al. Organs at risk in the brain and their dose-constraints in adults and in children: A radiation oncologist's guide for delineation in everyday practice. Radiother Oncol. 2015;1।4(2):230-238. doi:10.1016/j.radonc.2015.01.016

55. Andrews DW, Scott CB, Sperduto PW, et al. Whole brain radiation therapy with or without stereotactic radiosurgery boost for patients with one to three brain metastases: phase III results of the RTOG 9508 randomised trial. Lancet (London, England). 2004;363(9422): 1665-1672. doi:10.1016/ Sol40-6736(04)16250-8

56. Hasegawa T, Kondziolka D, Flickinger JC, Germanwala A, Lunsford LD. Brain Metastases Treated with Radiosurgery Alone: An Alternative to Whole Brain Radiotherapy? Neurosurgery. 2003;52(6):13181326. doi:I0.1227/0I.NEU.0000064569.189|4.DE

57. Nayak L, Lee EQ, Wen PY. Epidemiology of Brain Metastases. Curr Oncol Rep. 2012;14(I):48-54. doi:I0.1007/s| |912-011-0203-y

58. Berghoff AS, Schur S, Füreder LM, et al. Descriptive statistical analysis of a real life cohort of 2419 patients with brain metastases of solid cancers. ESMO open. 2016;1(2):e000024. doi:10.1136/ esmoopen-2015-000024

59. Cagney DN, Martin AM, Catalano PJ, et al. Incidence and prognosis of patients with brain metastases at diagnosis of systemic malignancy: a population-based study. Neuro Oncol. 2017;19(| I):I5II-I52|. doi:10.1093/neuonc/nox077 
60. Stalpers LJA, Dieleman EMT, van Westing BRH, Postma T], van Furth WR. [Diagnosis and treatment of brain tumours]. Ned Tijdschr Tandheelkd. 2009;/I6(4):202-207. http://www.ncbi.nlm.nih.gov/ pubmed/19438077.

61. Landelijke werkgroep neuro-oncologie. Landelijke richtlijn hersenmetastasen, versie 3.0. Oncoline. https://www.oncoline.nl/hersenmetastasen. Published 201l.

62. Schouten LJ, Rutten J, Huveneers HAM, Twijnstra A. Incidence of brain metastases in a cohort of patients with carcinoma of the breast, colon, kidney, and lung and melanoma. Cancer. 2002;94(I0):2698-2705. http://www.ncbi.nlm.nih.gov/pubmed/I2173339.

63. Nussbaum ES, Djalilian HR, Cho KH, Hall WA. Brain metastases. Histology, multiplicity, surgery, and survival. Cancer. 1996;78(8):I78I-I788. http://www.ncbi.nlm.nih.gov/pubmed/8859192.

64. Counsell CE, Collie DA, Grant R. Incidence of intracranial tumours in the Lothian region of Scotland, 1989-90. J Neurol Neurosurg Psychiatry. 1996;6I(2):I43-I50. http://www.ncbi.nlm.nih.gov/ pubmed/8708681.

65. Lagerwaard FJ, Levendag PC, Nowak PJ, Eijkenboom WM, Hanssens PE, Schmitz PI. Identification of prognostic factors in patients with brain metastases: a review of 1292 patients. Int J Radiat Oncol Biol Phys. 1999;43(4):795-803. http://www.ncbi.nlm.nih.gov/pubmed/l0098435.

66. Federatie Medisch Specialisten. Richtlijn hersenmetastasen. https://richtlijnendatabase.nl/richtlijn/ hersenmetastasen/startpagina_-_hersenmetastasen.html. Published 2020.

67. Yamamoto M, Serizawa T, Shuto $T$, et al. Stereotactic radiosurgery for patients with multiple brain metastases (JLGK090I): a multi-institutional prospective observational study. Lancet Oncol. 2014;15(4):387-395. doi:10.1016/SI470-2045(14)7006I-0

68. Soliman H, Das S, Larson DA, Sahgal A. Stereotactic radiosurgery (SRS) in the modern management of patients with brain metastases. Oncotarget. 2016;7(II):12318-12330. doi:10.18632/oncotarget.7I31

69. Mahajan A, Ahmed S, McAleer MF, et al. Post-operative stereotactic radiosurgery versus observation for completely resected brain metastases: a single-centre, randomised, controlled, phase 3 trial. Lancet Oncol. 2017;18(8):1040-1048. doi:I0.1016/SI470-2045(I7)304I4-X

70. Kocher M, Soffietti R, Abacioglu U, et al. Adjuvant whole-brain radiotherapy versus observation after radiosurgery or surgical resection of one to three cerebral metastases: results of the EORTC 22952-2600I study. J Clin Oncol. 2011;29(2):134-I4I. doi:I0.1200/JCO.2010.30.1655

71. Gaspar L, Scott C, Rotman M, et al. Recursive partitioning analysis (RPA) of prognostic factors in three radiation therapy oncology group (RTOG) brain metastases trials. Int J Radiat Oncol. 1997;37(4):74575I. doi:10.1016/S0360-3016(96)00619-0

72. Sperduto PW, Berkey B, Gaspar LE, Mehta M, Curran W. A New Prognostic Index and Comparison to Three Other Indices for Patients With Brain Metastases: An Analysis of I,960 Patients in the RTOG Database. Int J Radiat Oncol. 2008;70(2):5I0-5I4. doi:I0.1016/j.ijrobp.2007.06.074

73. Sperduto PW, Kased N, Roberge D, et al. Summary Report on the Graded Prognostic Assessment: An Accurate and Facile Diagnosis-Specific Tool to Estimate Survival for Patients With Brain Metastases. J Clin Oncol. 2012;30(4):419-425. doi:I0.1200/JCO.2011.38.0527

74. Sperduto PW, Deegan BJ, Li J, et al. Estimating survival for renal cell carcinoma patients with brain metastases: an update of the Renal Graded Prognostic Assessment tool. Neuro Oncol. July 2018. doi:10.1093/neuonc/noy099

75. Sperduto PW, Yang TJ, Beal K, et al. Estimating Survival in Patients With Lung Cancer and Brain Metastases: An Update of the Graded Prognostic Assessment for Lung Cancer Using Molecular Markers (Lung-molGPA). JAMA Oncol. 2017;3(6):827-831. doi:10.100I/jamaoncol.2016.3834

76. Sperduto PW, Jiang W, Brown PD, et al. Estimating Survival in Melanoma Patients With Brain Metastases: An Update of the Graded Prognostic Assessment for Melanoma Using Molecular Markers (Melanoma-molGPA). Int J Radiat Oncol. 2017;99(4):8I2-816. doi:10.1016/j.jijrobp.2017.06.2454

77. Sperduto PW. GPA Index. www.brainmetgpa.com. Published 2018.

78. Relton C, Torgerson D, O'Cathain A, Nicholl J. Rethinking pragmatic randomised controlled trials: introducing the "cohort multiple randomised controlled trial" design. BMJ. 2010;340:cl066. doi:10.1136/bmj.cl066

79. van der Velden JM, Verkooijen HM, Young-Afat DA, et al. The cohort multiple randomized controlled trial design: a valid and efficient alternative to pragmatic trials? Int J Epidemiol. 2017:46(I):96-I02. doi:10.1093/ije/dyw050 


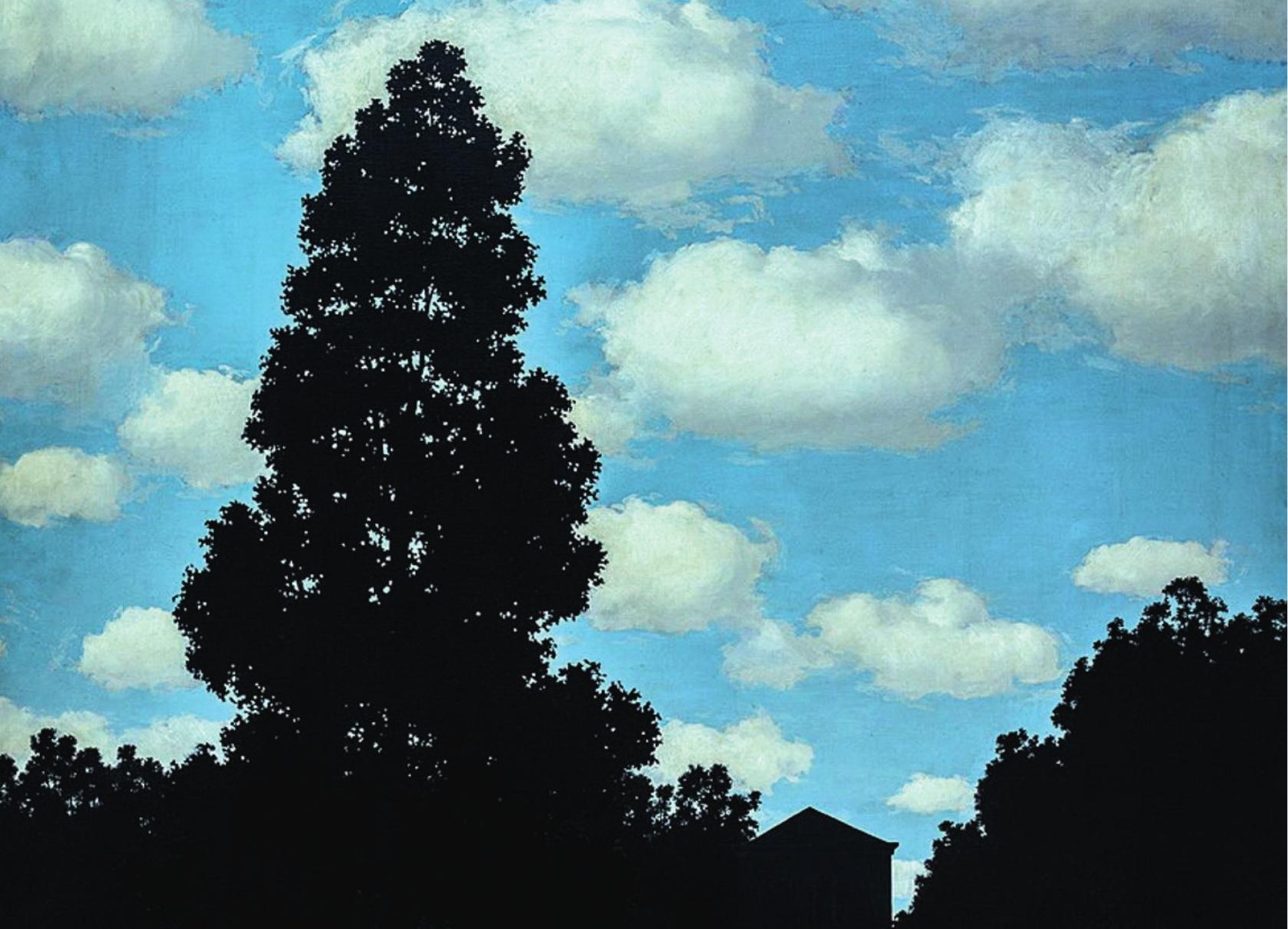

4t

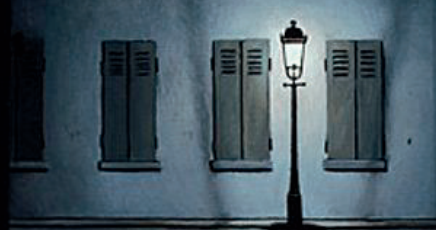




\section{Chapter 2}

Changes in cortical thickness and volume after cranial radiation treatment: a systematic review

S.H.J. Nagtegaal, S. David, A.T.J. van der Boog, A. Leemans, J.J.C. Verhoeff 


\section{ABSTRACT}

Cognitive decline has a clear impact on quality of life in patients who have received cranial radiation treatment. The pathophysiological process is most likely multifactorial, with a possible role for decreased cortical thickness and volume. As radiotherapy treatment systems are becoming more sophisticated, precise sparing of vulnerable regions and tissue is possible. This allows radiation oncologists to make treatment more patient-tailored. A systematic search was performed to collect and review all available evidence regarding the effect of cranial radiation treatment on cortical thickness and volume. We searched the Pubmed, Embase and Cochrane databases, with an additional reference check in the Scopus database. Studies that examined cortical changes on MRI within patients as well as between treated and non-treated patients were included. The quality of the studies was assessed with a checklist especially designed for this review. No meta-analysis was performed due to the lack of randomised trials. Out of 1915 publications twenty-one papers were selected, of which fifteen observed cortical changes after radiation therapy. Two papers reported radiation-dependent decrease in cortical thickness within patients one year after radiation treatment, suggesting a clear relation between the two. However, study quality was considered mostly suboptimal, and there was great inhomogeneity between the included studies. This means that, although there has been increasing interest in the effects of radiation treatment on cortex morphology, no reliable conclusion can be drawn based on the currently available evidence. This calls for more research, preferable with a sufficiently large patient population, and adequate methodology. 


\section{INTRODUCTION}

It is estimated that $50-90 \%$ of patients receiving radiotherapy (RT) to the brain experience progressive cognitive disability. ${ }^{1,2}$ The severity varies from mild impairment to symptoms similar to progressive dementia. The exact aetiology of this disabling consequence of tumour treatment remains unknown, but there are multiple hypotheses, including neuronal dysfunction, vascular damage and disturbed neurogenesis. The true cause is most likely multifactorial. ${ }^{1,3,4}$

Recent advances in imaging modalities and analyses have made testing of some of these hypotheses possible. ${ }^{5}$ Although most imaging studies focus on the relationship between radiation-induced cognitive defects and white matter (WM) $)^{6,7}$ or the hippocampus ${ }^{8,9}$, increasing attention has been given to the changes in the cerebral (neo)cortex. The cerebral cortex contains a vast number of neurons, and has a thickness between I and $4.5 \mathrm{~mm}$ in vivo, depending on location..$^{10}$ The neurons are interconnected in an immensely complex network and form the tissue which is essential for the execution of all cognitive processes. ${ }^{\prime \prime}$ It is comprised of both neuronal cell bodies and fibres, together with glial cells and blood vessels. ${ }^{12}$ Both damage to neurons and vasculature can lead to disruption in the cortical network, and have been linked to changes in cognitive abilities., ${ }^{4,13}$

Before the era of digital image analysis, macroscopic cortical atrophy as seen on routine CT or MRI had already been associated with RT and cognitive function. ${ }^{14-17}$ But now, the exact thickness of the cerebral cortex throughout the brain can be accurately computed from MR-images using specific software.10,18-24 There are several ways to perform the morphological measurements, including deformation-based, voxel-based and surfacebased morphometry (DBM, VBM and SBM), with the latter allowing for measurement of the cortical thickness. ${ }^{25}$ In this technique, the surface of the brain is recreated as a fine mesh, and the distance between the surface and the border between the cortex and the underlying WM is calculated for each point in this mesh. The thickness is measured on a sub-millimetre level, which allows researchers to detect even microscopic changes between multiple scans. Although decrease in cortical thickness is part of the normal aging process, ${ }^{26}$ it has also been linked with several diseases, including Alzheimer's disease ${ }^{5,27}$, Parkinson's disease $^{28}$ and depression. ${ }^{29,30}$ Furthermore, it has also been associated with cognitive impairments, ${ }^{31,32}$ which leads to the hypothesis that cortical thinning is a contributing factor to the impairments seen after radiation therapy. This is further supported by the fact that animal models have shown that irradiation during the pre-natal developmental period can also contribute to cortical abnormalities, including the thickness and volume of the cortex. ${ }^{33-35}$ 
Aside from cortical thickness, cortical volume can also be derived from MR-images using the VBM method mentioned above. It is a more straightforward technique than the more complex SBM, which allows for quicker analysis. It classifies the voxels of an MRI-scan according to tissue types (grey matter, white matter or cerebrospinal fluid), quantifying the amount of each tissue type in each voxel. These values are added up to get the volume of each tissue. While the two measures are related, as the volume of the cortex changes along with its thickness, it is still recommended that both metrics should be interpreted separately due to their different genetic origins. ${ }^{36}$

To gather all available current evidence regarding the effect of cranial RT on the cerebral cortex, we have conducted a systematic review of the literature. In this review we attempt to answer the following question: what evidence exists that changes in cortical thickness and volume occur in patients who have received cranial irradiation?

\section{METHODS}

\section{Search strategy}

The systematic review was conducted according to the PRISMA (Preferred Reporting Items for Systematic Reviews and Meta-Analyses) Statement. ${ }^{37}$ A search was performed in the Pubmed, Embase and Cochrane databases on the $21^{\text {st }}$ of March 2018, and was constructed with help of a research librarian at Utrecht University, specialised in medical systematic reviews. The full search strings for each database are found in Appendix I. In short, we searched with terms relating to radiation therapy and cortical thickness or volume. The references of the ultimately selected studies were checked for additional studies in the Scopus database.

\section{Study selection}

After duplicate removal, all titles and abstracts of the search results were independently screened by 2 reviewers (SN and AvdB), and selected based on the inclusion criteria presented in Figure I. We were primarily interested in studies comparing cortical changes after RT within the same patients. However, in order to get a complete overview of the effect of RT, we also selected studies that performed a cross-sectional comparison between patients who received RT and non-RT controls. Therefore, both study designs were part of the inclusion criteria. The screening of the studies was facilitated by Covidence systematic review software (Veritas Health Innovation, Melbourne, Australia). Conflicts in screening were resolved during a consensus meeting. After screening, the full texts of the remaining manuscripts were read and the final selection of studies was made, again independently by the two screening authors, followed by another consensus meeting to resolve conflicts. 


\section{Data extraction}

Data was extracted by the first author using a standard data collection form. We collected any analyses of changes or differences in either cortical thickness or volume after RT. If present, the relation between RT dose and cortical changes was also collected. Additionally, data on population, sample size, type of RT and length of follow-up were collected. When only part of the population of a study received $\mathrm{RT}$, and no RT-subgroup analysis was reported, data from the entire population were extracted. Finally, if a study analysed the volume of the entire grey matter instead of the cerebral cortex, this difference in grey matter volume was extracted.

\section{Data quality and relevancy}

A checklist was constructed to assess data quality and relevancy of the papers. The domains of this checklist included case selection, study design and analysis quality. A complete overview of the assessment checklist, and the criteria that needed to be met for a positive score, are shown in Table I.

The criteria in the domain "case selection" were mainly chosen to reflect on the risk of selection bias in the studies. The description of the way cases are selected, whether a random or consecutive sample was used, and a description of excluded and nonparticipating subjects allow us to verify that all eligible patients were included in the study. A comprehensive case description allows readers to see how the patients and controls relate to each other, and whether or not other treatments have been given to one or all groups.

The study design was evaluated by determining whether a priori brain regions were analysed. The use of these regions of interest (ROl's) means that the researchers identified brain regions where cortical changes are expected to occur. They were selected because these areas are related to certain cognitive functions or these areas have been shown to be vulnerable in earlier research. Using a limited number of a priori brain regions also negates the problem of multiple comparisons, whereby a significant result is more easily obtained when analysing many different brain regions simultaneously. Dividing the cortex into regions for analysis, as opposed to complete brain or lobes, means smaller areas of cortical thickness and volume change can be found. Similarly, only an analysis of the complete brain results in finding all possible regions of cortical change. Studying the relation between cortical change and dose shows that the observed changes are more likely to be related to radiation therapy, and are less likely to be caused by other factors, like aging, which is well described. ${ }^{26}$

Finally, the quality of the imaging and processing procedures and the quality of analysis and its description gives an indication of the methodological and statistical validity, and thereby the risk of bias by confounding or multiple comparisons. 


\section{RESULTS}

After duplicate removal, the search yielded a total of 1915 unique studies. After screening of title and abstract, 63 studies were selected for full text evaluation. After reading the full text, 21 studies were included for this review. A reference check in Scopus revealed no additional studies. The flow-chart of study selection in shown in Figure I.

Of the selected studies, 7 reported on cortical thickness after radiation therapy. Thirteen measured either cortical or grey matter volume after RT, and one studied both thickness and volume. An overview of the studies is found in Table $\mathbf{2}$ and Table 3, and the assessment of bias and relevancy are found in Table 4. As none of the found studies are randomized controlled trials, meta-analysis of the study results is not advised. ${ }^{38}$ Therefore, the findings of the found references will be presented in a narrative manner.

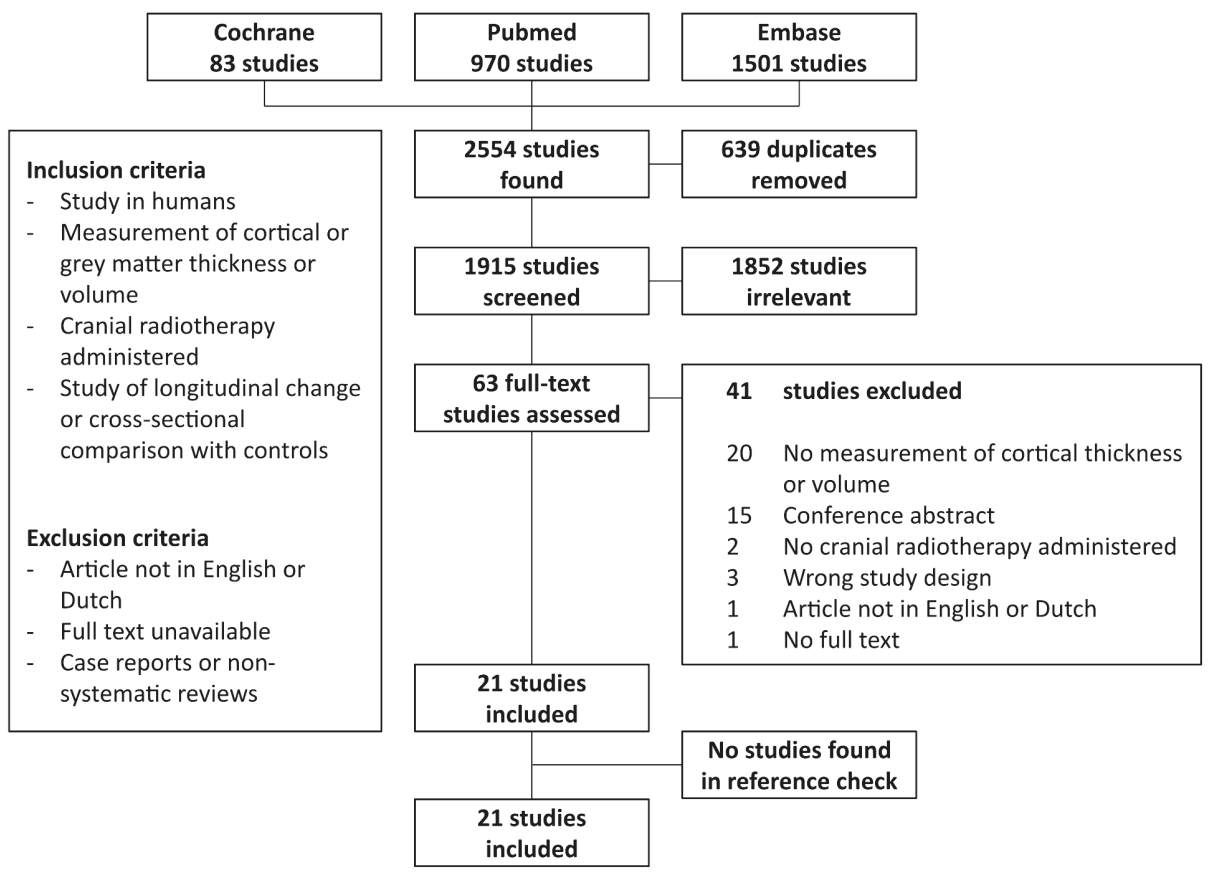

Figure I Flow-chart of article selection, and overview of inclusion and exclusion criteria 
Table I Criteria for assessing risk of bias and study relevancy

\begin{tabular}{|c|c|c|}
\hline & Yes & No \\
\hline $\begin{array}{l}\text { Case selection } \\
\text { described }\end{array}$ & $\begin{array}{l}\text { Setting, time frame and eligibility } \\
\text { criteria for recruitment provided }\end{array}$ & $\begin{array}{l}\text { Data on setting, method of } \\
\text { selection and eligibility criteria for } \\
\text { recruitment incomplete }\end{array}$ \\
\hline $\begin{array}{l}\text { Random or } \\
\text { consecutive } \\
\text { sample }\end{array}$ & $\begin{array}{l}\text { Consecutive or random participant } \\
\text { sampling }\end{array}$ & $\begin{array}{l}\text { No consecutive or random } \\
\text { participant sampling or unclearly } \\
\text { described }\end{array}$ \\
\hline $\begin{array}{l}\text { Non- } \\
\text { participants }\end{array}$ & $\begin{array}{l}\text { Data on non-participating (excluded } \\
\text { patients in case of retrospective } \\
\text { study, eligible but non-participating in } \\
\text { case of prospective study) subjects } \\
\text { given, including reason for non- } \\
\text { participating. }\end{array}$ & $\begin{array}{l}\text { Data on non-participating subjects } \\
\text { not reported or incomplete }\end{array}$ \\
\hline $\begin{array}{l}\text { Case } \\
\text { description }\end{array}$ & $\begin{array}{l}\text { Information on participants complete } \\
\text { (demographics, primary disease, } \\
\text { received treatments) }\end{array}$ & Data on participants incomplete \\
\hline $\begin{array}{l}\text { A priori brain } \\
\text { regions }\end{array}$ & $\begin{array}{l}\text { Pre-defined brain regions of interest } \\
\text { studied }\end{array}$ & $\begin{array}{l}\text { No pre-defined brain regions of } \\
\text { interest studied }\end{array}$ \\
\hline $\begin{array}{l}\text { Discrete RT } \\
\text { patient group }\end{array}$ & $\begin{array}{l}\text { Results are available of a group of } \\
\text { patients that all received RT }\end{array}$ & $\begin{array}{l}\text { Data available only of combined } \\
\text { treatment group }\end{array}$ \\
\hline $\begin{array}{l}\text { Changes in } \\
\text { cortex regions }\end{array}$ & $\begin{array}{l}\text { Changes in cerebral cortical regions } \\
\text { measured and reported }\end{array}$ & $\begin{array}{l}\text { Changes in total cerebral lobes, } \\
\text { total cortex or total grey matter } \\
\text { measured }\end{array}$ \\
\hline $\begin{array}{l}\text { Relation to } \\
\text { dose }\end{array}$ & $\begin{array}{l}\text { Changes in cortex are relation to RT } \\
\text { dose and location }\end{array}$ & $\begin{array}{l}\text { Dose and location of } R T \text { is not } \\
\text { related to cortical changes }\end{array}$ \\
\hline $\begin{array}{l}\text { Compete brain } \\
\text { analysed }\end{array}$ & $\begin{array}{l}\text { Cortical changes measured in entire } \\
\text { brain, or entire brain with tumour } \\
\text { regions censored }\end{array}$ & $\begin{array}{l}\text { Cortical changes not measured in } \\
\text { entire brain, e.g. in one cerebral } \\
\text { lobe }\end{array}$ \\
\hline $\begin{array}{l}\text { Analysis } \\
\text { described }\end{array}$ & $\begin{array}{l}\text { Methods of image analysis and } \\
\text { computation adequately described, } \\
\text { including imaging parameters and } \\
\text { software used }\end{array}$ & $\begin{array}{l}\text { Methods unclearly or incompletely } \\
\text { described }\end{array}$ \\
\hline $\begin{array}{l}\text { Analysis } \\
\text { quality }\end{array}$ & $\begin{array}{l}\text { Use of correction for multiple } \\
\text { comparisons, nonparametric } \\
\text { statistical inference and controlling } \\
\text { for possible confounding factors }\end{array}$ & $\begin{array}{l}\text { One or more items missing; or the } \\
\text { description is unclear }\end{array}$ \\
\hline
\end{tabular}




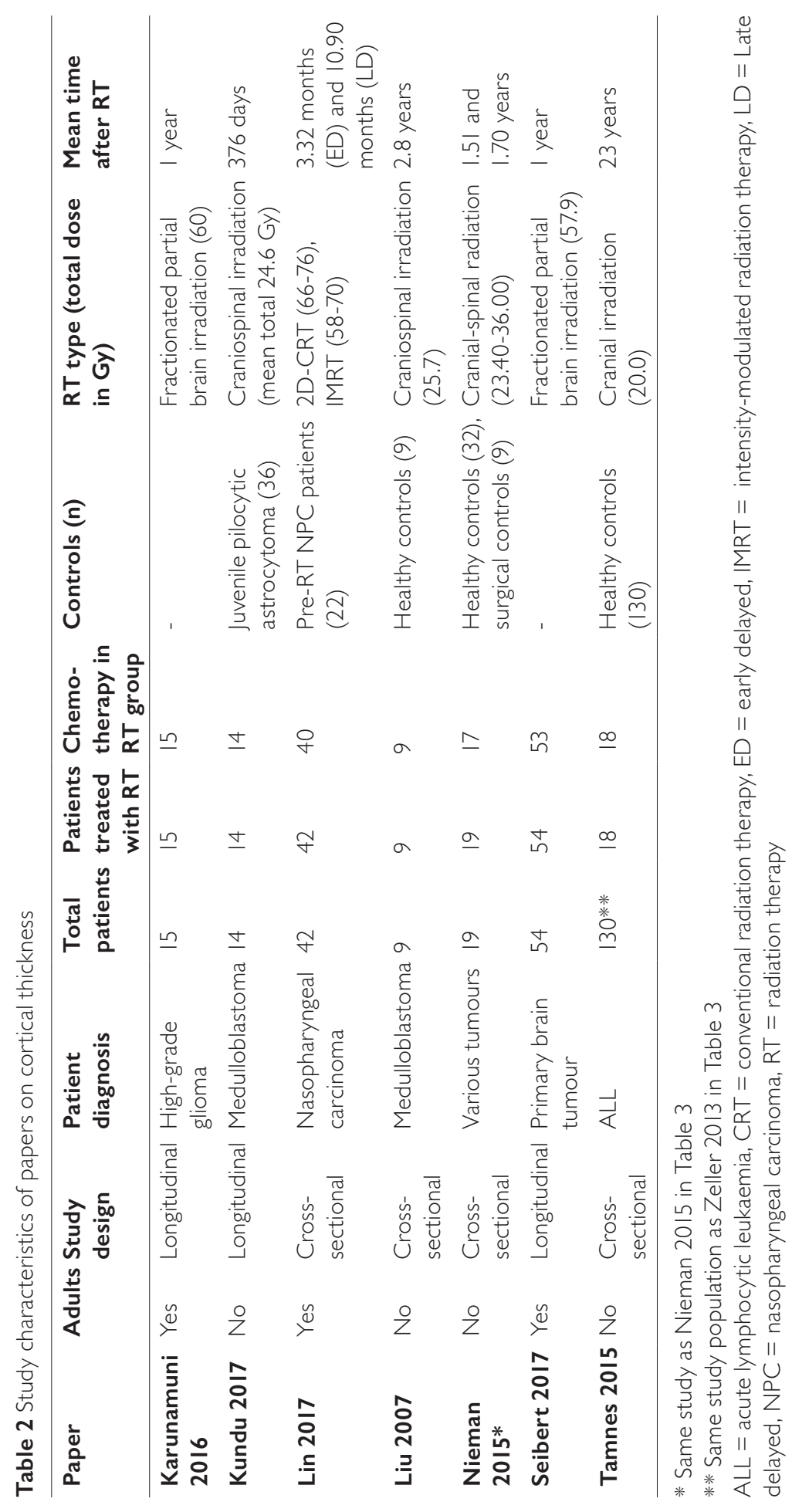




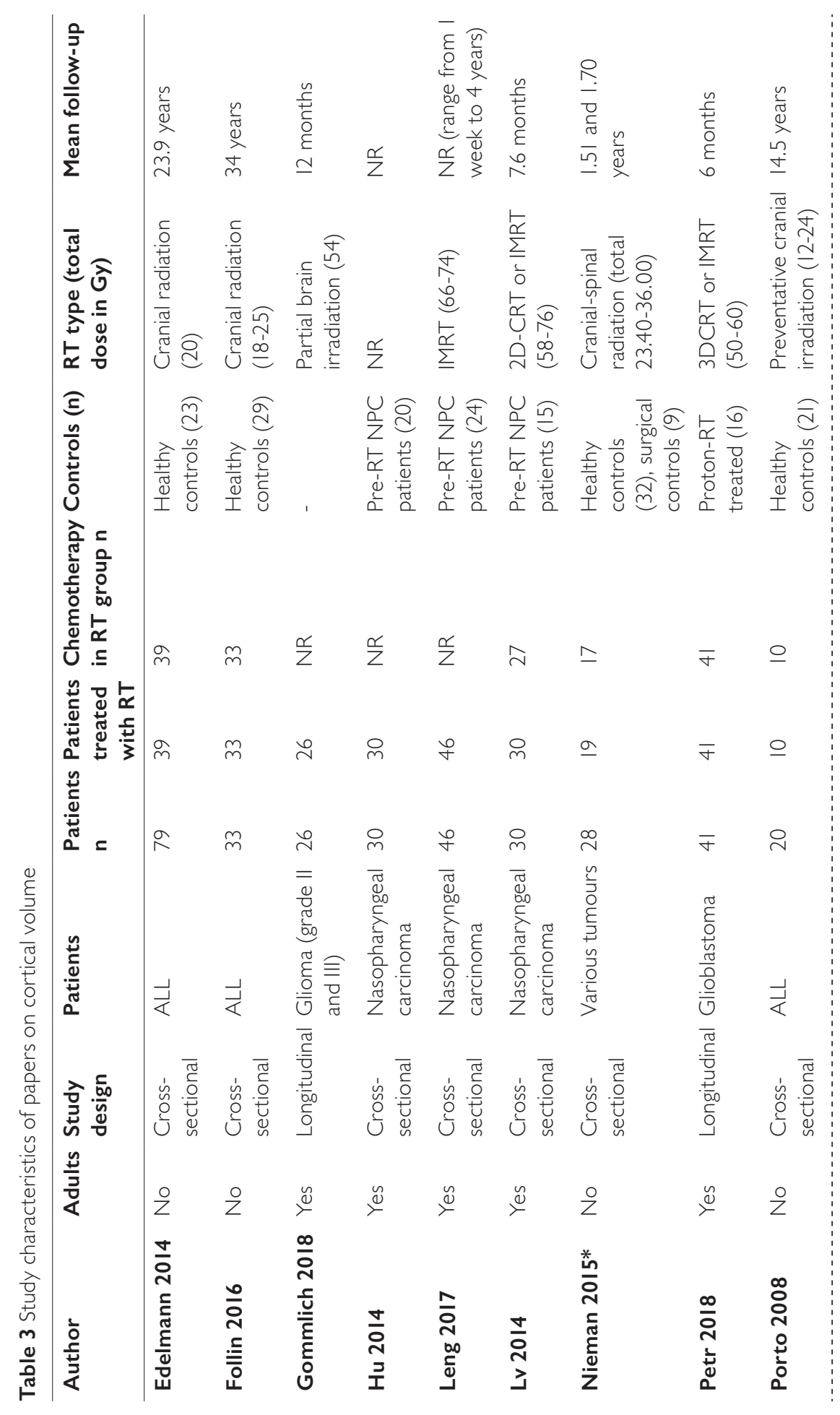




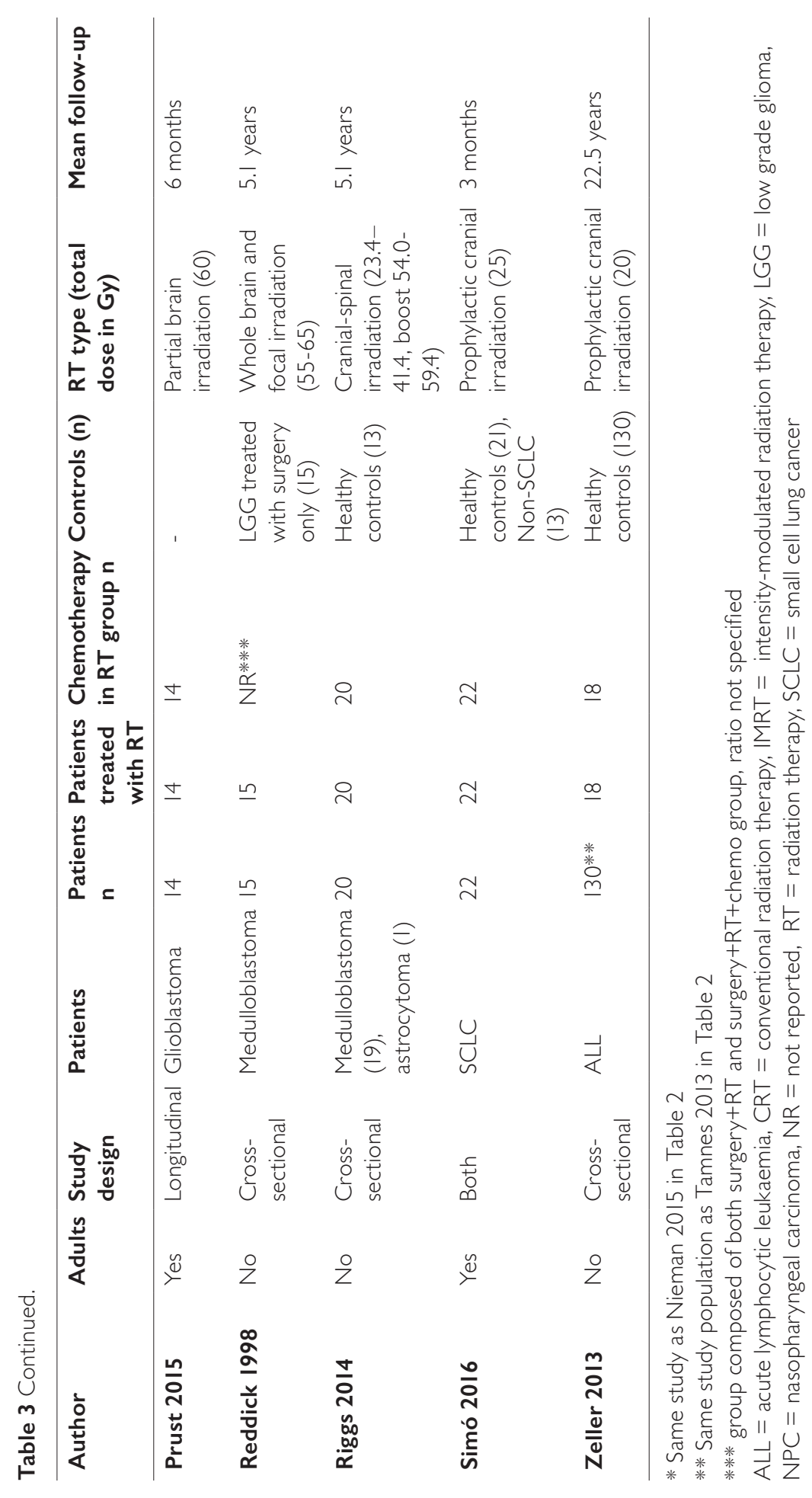




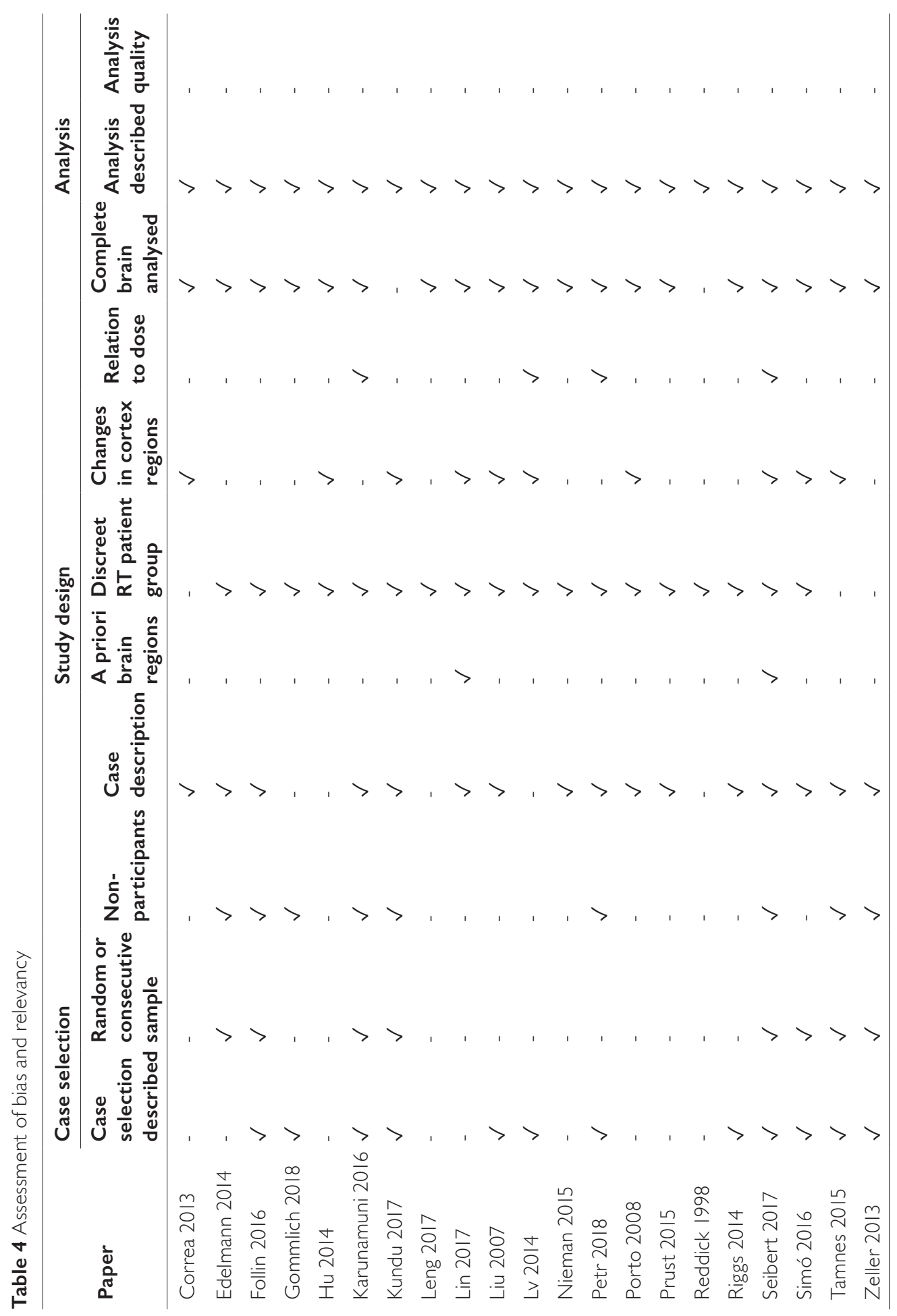




\section{Cortical thickness}

Eight studies presented data on the effect of radiation therapy on cortical thickness. Of those, three studies registered longitudinal changes within patients, four cross-sectional studies compared RT patients with non-RT controls (either matched healthy controls or patients who received other cancer treatments), and one study combined a longitudinal and a cross-sectional design.

\section{Longitudinal studies}

Two studies scored highest in our assessment tool and both investigated dose-dependent cortical thinning in a longitudinal design by comparing pre-RT MR images with those made one year after treatment.

Karunamuni et al retrospectively studied 15 high grade glioma patients who underwent fractionated partial brain RT and chemotherapy. ${ }^{39}$ They concluded that total cortical thickness decreased more with increasing doses of administered RT. With every additional Gy the cortex showed thinning of $1.3 \mu \mathrm{m}$, up to a total dose of $34.6 \mathrm{~Gy}$. Above this dose, thinning increased to $7.2 \mu \mathrm{m} / \mathrm{G} y$. They further analysed the cortical thickness per lobe, and found that the parietal, limbic and temporal lobes were most susceptible to cortical thinning after RT, the latter being the most vulnerable.

Seibert et al conducted a similar study, but used a pre-specified $\mathrm{ROI}$ analysis. ${ }^{40}$ They selected 54 patients treated with RT for primary brain tumours, and looked at the effect of regional dose on cortical thickness. They focussed their attention on cortical regions associated with higher cognitive functions: the inferior parietal cortex and the entorhinal cortex (see Figure 2). These two ROl's were compared with two regions representing the primary cortex: the pericalcarine cortex and the paracentral lobule. They found a significant correlation between radiation dose and the amount of thinning in their two regions of interest, whereas no such correlation was found in the two reference regions. When assessing whether cortical reduction appears in areas receiving either low (<20 Gy) or high (>40 Gy) mean dose, they found that the two ROl's combined showed significant loss of thickness of 0.19 $\mathrm{mm}(6 \%)$ after receiving the high dose. Again, this was not found in the primary cortex. Additionally, they used a linear mixed-effects model to test the effect of regional radiation dose to cortical thinning for each of the 34 cortical regions of the Desikan-Killiany brain atlas. This showed radiation vulnerability in 9 brain regions, with cortical thinning ranging from $1.8 \mu \mathrm{m}$ to $6.5 \mu \mathrm{m}$ per Gy. 


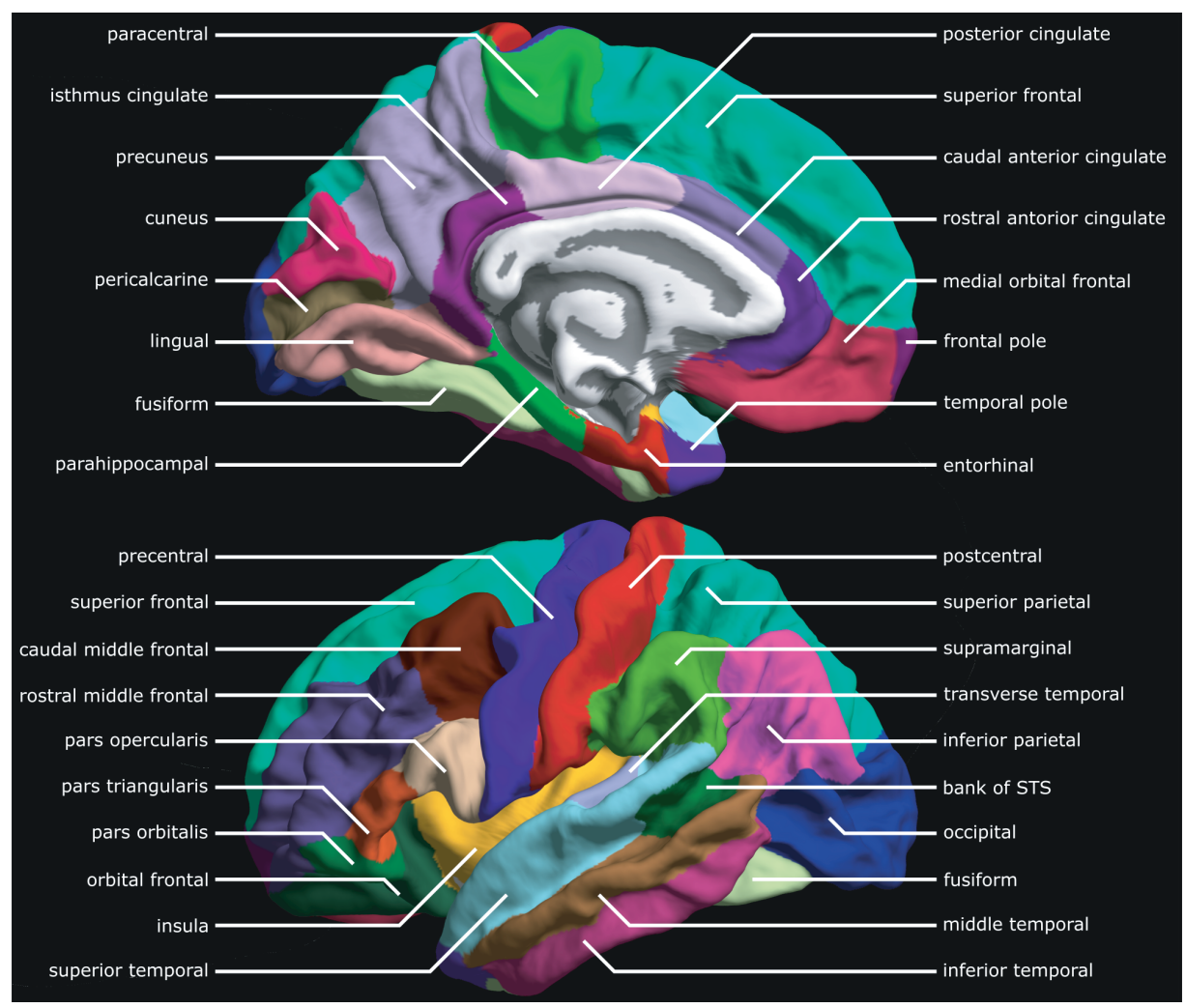

Figure 2 Cortical regions from the Desikan-Killiany ${ }^{85}$ atlas presented on the central surface of the template mesh-brain via the Computational Anatomy Toolbox (CATI2) $)^{18,86}$.

Two other studies exploring longitudinal change in cortical thickness were performed by Correa et al. and Kundu et al. ${ }^{41,42}$ They both had a paediatric study population, with hematopoietic stem cell transplantation recipients and medulloblastoma patients respectively. Kundu et al. found an increase in temporal cortical thickness I year after RT. They compared this to juvenile pilocytic astrocytoma patients who received only surgical treatment, and who showed cortical thinning normal for their age. Correa et al. saw a significant cortical thinning in the left and right middle frontal gyrus in their subjects. However, they used a study population that was largely comprised of patients who received only chemotherapy, with only some receiving chemoradiation. There was not enough statistical power to analyse the effect of RT alone. Analysing the chemotherapy group revealed decrease of the left middle frontal gyrus, but no significant changes in the right middle frontal gyrus. 


\section{Cross-sectional studies}

Of the cross-sectional studies, the one by Lin et al. measured post-RT cortical thickness in adult patients. ${ }^{43}$ They divided a cohort of nasopharyngeal carcinoma patients into three: pre-RT, I-6 months post-RT (named ED: early delayed) and 7-18 months post-RT (LD: late delayed). They compared cortical thickness between these groups in both vertex-wise and ROI-wise analyses. The latter was done by dividing the brain into I48 ROI's according to the Destrieux brain atlas. Comparing the pre-RT group to the post-RT-ED groups revealed one vertex cluster of thinner cortex located in the left precentral gyrus of the treated patients. However, when comparing the cortical thickness of the pre-RT to the post-RT-LD, they found a thicker cortex in the latter group in 5 vertex clusters and 22 ROl's, spread across all cerebral lobes and both hemispheres. The cortex in these ROl's showed a relative thickness difference between $4.4 \%$ and $12.8 \%$. The dose and location of radiation was not taken into account in these analyses, so it is not certain whether or not the areas showing cortical differences were subjected to irradiation. Although age and gender were included as covariates in the $\mathrm{ROI}$-wise analysis, pre-existing cortical differences between the two patient groups can be the explanation for some or all found differences. Additionally, patients were treated with different radiation techniques (2D-CRT or IMRT), adding to the inhomogeneity of the study population.

The three remaining cross-sectional studies all described a paediatric population who received both RT and chemotherapy. Two studies by Tamnes et al. and Liu et al. compared survivors of childhood malignancies to healthy controls, and found I and 5 brain regions with a significantly thinner cortex, respectively. ${ }^{44,45}$ However, the former study only had 18 patients treated with RT in their 130-patient study population, and the latter included 9 patients only, all treated with both RT and chemotherapy. Nieman et al. found a thicker cortex in the frontal, occipital and temporal lobes in their $>7$ year-old group. ${ }^{46}$ Finally, Correa et al. performed cross-sectional analysis in addition to their longitudinal one, but found no significant differences. ${ }^{41}$

\section{Cortical volume}

Of the papers on cortical volume changes after radiotherapy, three had a longitudinal design, ten were cross-sectional, and one did both.

\section{Longitudinal studies}

The best level of evidence, according to our assessment tool, was found in the paper by Simó et al. They studied the changes in grey matter volume in 22 small cell lung cancer (SCLC) patients receiving prophylactic cranial irradiation. ${ }^{47}$ They found a significant volume reduction of the bilateral insular cortex, right parahippocampal gyrus and superior and temporal middle gyrus three months post-radiation. These longitudinal changes were not 
found in either healthy controls or non-small cell lung cancer (NSCLC) patients who did not undergo radiation therapy. This difference is unlikely to be caused by systemic therapy, as both SCLC and NSCLC groups received comparable chemotherapy regimens. Additionally, they performed neuropsychological tests on these three groups, and found that the SCLC patients performed worse in several domains, including verbal fluency and processing speed. No attempts were made to correlate these cognitive deficits to radiation dose or cortical volume reduction. In a previous study by the same research group, similar grey matter (GM) changes were observed I month after starting platinum-based chemotherapy, before cranial irradiation was started. ${ }^{48}$ As the SCLC patients who received cranial irradiation also underwent this chemotherapy regimen, the authors suggest the GM change is most likely the result of chemotherapy. However, as stated above, no longitudinal changes were observed in the NSCLC patients, who underwent chemotherapy only. Furthermore, group by time analysis of cortical volumes between the two groups found significant differences in GM volume change in several brain areas.

In a study by Petr et al., the effects of radiotherapy on brain matter volumes of the healthy hemisphere was compared between patients receiving photon and proton therapy for glioblastoma. ${ }^{49}$ In the photon group, which included 4 I patients, a loss of total GM volume was seen after 3 and 6 months. Furthermore, they found that GM volume loss increased with mean radiation dose, with areas receiving $>10$ Gy showing more volume loss when compared to those receiving $<10$ Gy $(-2.4 \%$ and $-1.6 \%$, respectively). Further stratification of dose revealed highest volume loss in the regions that received 30-60 Gy, namely -3.1\%. In multivariate linear regression mean received dose had a significant effect on GM volume of $0.9 \% / / 0 G y$. Prust et al. also found a loss of GM volume after chemoradiation for glioblastoma. ${ }^{50}$ This study only included 8 patients who were eligible for image analysis, and data at last follow-up (35 weeks) was available for only 3 participants. Also note that these last two studies measured GM volume as a whole, which not only includes cerebral cortex, but also the thalamus and basal ganglia.

The final longitudinal study, by Gommlich et al., found no significant change in 14 glioblastoma patients who received radiation therapy. ${ }^{51}$

\section{Cross-sectional studies}

Of the cross-sectional studies, three had an adult study population, all treated with radiation therapy for nasopharyngeal carcinoma (NPC). Lv et al. found a smaller cortical volume in several areas in the temporal and frontal lobes in treated NPC patients compared to pretreatment patients. ${ }^{52}$ They also found a negative correlation between the mean dose to the temporal lobes and cortical volume of select areas therein. Similarly, Leng et al. found numerous areas of smaller volume in all follow-up groups (<6 months, 6-12 months and 
$>12$ months after RT). ${ }^{53} \mathrm{Hu}$ et al. found a smaller volume of the right paracentral lobule in patients treated in the last 6 months compared to pre-treatment controls. ${ }^{54}$ However, in their delayed reaction group (7-24 months) they observed a bigger volume of this area compared to the early treatment group.

Edelmann et al. found a smaller GM volume of the parietal and temporal lobes in acute lymphoblastic leukaemia (ALL) survivors who received cranial RT in childhood compared to those who underwent chemotherapy only. ${ }^{55}$ Follin et al. and Zeller et al. both found smaller total GM volume and total cortical volume, respectively, in ALL patients compared to healthy controls. ${ }^{56,57}$ However, when correcting for total intracranial volume, Follin et al.'s result lost significance. Zeller et al. used the same study population as Tamnes et al. which, as mentioned above, only had a small fraction of RT patients in their patient group. When analysing the effect of treatment variables on GM volume, they found no significant correlation. They also subjected the participants to neuropsychological testing, and found a correlation between cortical GM volume and processing speed as well as executive function. This correlation lost its significance when corrected for multiple comparisons.

Finally, Nieman et al., Porto et al., Reddick et al. and Riggs et al. found no significant differences in their RT population compared to controls. ${ }^{46,58-60}$

\section{DISCUSSION}

In this systematic review we set out to find all available evidence regarding the effects of radiation therapy on cortical thickness and volume. From 1915 manuscripts we included 21 studies, of which 7 studied cortical thickness, 13 studied cortical or grey matter volume after RT, and I studied both.

Fifteen papers seemed to show at least some degree of cortical change, with Karunamuni et al. and Seibert et al. providing the most reliable evidence as scored by our checklist (Tables I and 4). They correlated longitudinal cortical change to radiation dose, and found that cortical thickness decreases with every additional Gy delivered to healthy brain tissue in select brain regions. Furthermore, Karunamuni et al. found a threshold of 34.6 Gy above which cortical thinning increases. Similarly, Petr et al. found an increase in GM volume loss with higher total doses of RT. These findings are in line with cortical atrophy and other gross morphological changes seen in the cortex in older imaging studies ${ }^{14-17}$ and studies of pre-natal radiation exposure in animals. ${ }^{33,34,61}$ The exact biological process that leads to this visible loss of cortical thickness and volume remains unknown. There is evidence that, as part of a complex inflammatory reaction, phagocytosis of healthy and damaged neurons occurs after radiation though activated microglia. ${ }^{62}$ This atrophy of neural cells is 
likely to be a contributor to loss the diminished cortex, along with the loss of glial cells. ${ }^{63}$ Another histopathological change often seen in the cortex after radiation therapy is vascular damage, ${ }^{1,64}$ a potential consequence of which is demyelination of the cortex. Demyelination is a known effect of vascular damage in WM, ${ }^{1,4}$ and although myelin is most abundant in WM, the cortex also contains myelinated axons. ${ }^{65}$ Loss of myelination in the cortex could lead to a decrease of cortical volume, and thereby also affect thickness. Demyelination, as well as glial atrophy, have already been observed in radiation damage in mice and rats. ${ }^{66}$ Several of our selected papers examined additional outcomes that reflect these structural changes, like those by Petr et al., who looked at grey matter perfusion and Edelman et al., who performed diffusion tensor imaging (DTI).

In contrast, Lin et al. found select regions of a thicker cortex in their late delayed (7-18 months post-RT) patient group compared to the pre-RT group. This difference could simply be explained due to pre-existent differences between the two patient groups, but an effect of RT is also possible. The authors suggest that the thicker cortex post-RT could be caused by astrogliosis, which is a known long-term effect of brain irradiation. ${ }^{67}$

Another hypothesis for changes in cortical morphology we would like to pose, and which we have not seen discussed before, has to do with the border between GM and WM. As radiation leads to demyelination and myelin is the main component of WM, we hypothesise that the boundary between white and grey matter could become less defined. As the WM contains more myelin than GM, loss of demyelination could affect the WM more than the cortex, which might cause the perceived GM/WM border to shift toward the white matter. The method of cortical thickness estimation starts with segmentation into WM and GM, so when the border shifts, results of cortical measurement change accordingly. As the changes are mostly measured on sub-millimetre level, even the smallest shifts in the GM/ WM border can lead to significant changes in perceived morphology. Supportive of this hypothesis is the fact that demyelinated WM appears hypointense on TI weighted MRI, ${ }^{68}$ which is the same imaging sequence that is used for cortical morphology measurements.

Even the papers that scored highest in our assessment tool did not meet all our criteria for a methodologically sound paper. In fact, none of the found papers used the statistical methods which would meet the requirements of modern neuroimaging standards: multiple comparison correction, nonparametric statistical inference and controlling for possible confounding factors. For instance, analysing the change in cortical thickness on a voxelwise or vertex-wise basis means that for each patient more than 100,000 data points are used for analysis. For each of these data points a statistical test is performed, and using the usual 0.05 threshold for $p$-values results in $5 \%$ of the 100,000 tests giving in a false positive result, even if the null hypothesis is really true. The resulting 5000 false positive voxels are 
large enough to interpret as meaningful finding, while they are actually a mere consequence of the testing setup. In other words, when not correcting for these multiple comparisons, this may lead to a false significance of the observed effect of radiation on the cerebral cortex. Examples of multiple comparison corrections are the false discovery rate (FDR) ${ }^{69}$ or the control of the voxel-level family-wise error rate (FWE)..$^{70}$ Other studies used the Desikan-Killiany or Destrieux brain atlases, which are comprised of 34 and 74 brain areas respectively. This vast difference in data quantity means the power of the study can increase based on the method used, while keeping the same number of patients. This problem can be overcome with adequate statistical methods, but these have been largely unused in the studies we found. And even when the significance of an observed change is legitimate, it remains unclear which effect size has any meaningful consequences. A change of several micrometres may be significant, but its effect on cognitive symptoms may be negligible. So the question remains how clinically relevant these results are.

Aside from these statistical objections, the way cases were selected and reported was also found lacking in fifteen papers according to our assessment tool. Using a non-consecutive or non-random patient sample is accompanied by the risk of selection bias. This might be a particularly important factor in papers that use a retrospective cohort and are unclear in their method of patient selection.

The biggest limitation to this systematic review is the great inhomogeneity between the selected papers, in both study design and quality. No two papers had similar study designs, patient population and follow-up time. Other major differences are sample size (ranging from 5 to 130), RT type and dose, and type of controls in the cross-sectional studies. These differences make comparing papers difficult, and an attempt at meta-analysis unjustifiable.

There are several additional methodological issues with the selected studies. Eight papers discussed cortical changes after treatment during childhood, with follow-up times varying between 3 months and 34 years. During normal brain maturation and development, changes are seen in the thickness of the cerebral cortex. Whether the cortex decreases from birth or undergoes a period of thickening before it reduces is still an on-going debate, in which no consensus had been reached. ${ }^{7 l}$ Either way, the effect of this normal biological process cannot be easily separated from the effect of radiation therapy, which also means the results of these studies cannot be reliably be interpreted, and certainly cannot be extrapolated to an adult population.

More than half of the included studies did not measure differences in cerebral cortex between pre- and post-RT scans of the same patients, but instead applied a cross-sectional design. They compared the cortical thickness or volume of RT patients with either healthy 
controls or non-RT patients. Most of these controls were age- and gender matched, eliminating the influence these two factors have on the cerebral cortex. ${ }^{26,72}$ However, other factors such as handedness, genetics, pregnancy and even socio-economic status have also been related to differences in the cerebral cortex. ${ }^{73-76}$ The precise biological processes underlying these differences is not known, but the effect of sex and pregnancy suggests a hormonal component. ${ }^{76}$ As these factors are usually not taken into account when using matched controls, pre-existent differences between patients may explain some or all of the results found in the cross-sectional papers. In a study measuring the cortical thickness in 30 healthy patients, a mean standard deviation of $0.54 \mathrm{~mm}$ was found..$^{10}$ This means that, with an average thickness of $2.7 \mathrm{~mm}$, the cortex can be expected to differ by more than $20 \%$ in around one third of cases. The interpersonal difference in cortical thickness is even greater in the association areas, namely the prefrontal and temporal cortices. ${ }^{10}$

Another important factor in these studies is the administration of chemotherapy. This treatment has also been linked to changes in the cerebral cortex.77,78 As mentioned before, not all papers incorporated this confounding factor in their analyses, which may influence the reliability of their results. Furthermore, some studies had a "treated" and an "untreated" group, whereby only a fraction of patients in the treated group received RT, and most received only chemotherapy. This means that no conclusion about the effect of radiation therapy can be drawn.

Finally, also publication bias by 'file drawer effect' might play a role in studies on the effect of RT.79 As it is not customary to publish a research protocol, or to otherwise make it known this effect is being studied, we do not know how many studies have not been reported due to inconclusive or negative results. It is possible more research groups did similar tests, but found no difference before and after RT, and therefore decide did not publish their findings. We urge future researchers performing a high-quality study and finding a negative result to still publish their findings, because absence of a relation between RT and cortical damage is also valuable knowledge to have. We are unable to create a funnel plot to test for publication bias, for the same reasons a meta-analysis could not be performed.

All these limitations, especially the great inhomogeneity and consequential unjustifiability of meta-analysis, mean that we cannot reliably claim that RT does indeed affect the thickness or volume of the cerebral cortex based on evidence that is currently available. Similarly, we are unable to point to cortical regions that are especially susceptible to radiation damage.

Suggestions for future research are clear: there is a need for larger studies, with sound methodology. Ideally, studies should also try to correlate radiation dose, longitudinal cortical change and cognitive deficits within the same patient group. The recommendation of this 
review is to test this association, which may lead to the identification of cortical areas especially vulnerable to radiation-induced cognitive decline. If such areas do indeed exist, we may avoid them during radiation therapy, the same way we now are able to do for the hippocampus. ${ }^{80}$ Identification of these areas at risk are especially useful for newer, more precise radiation therapy techniques like the MR-Linac, which combines a 1.5T MRI scanner with a linear accelerator. ${ }^{81}$ With this combination, it becomes possible to correct for patient movements, and to adapt treatment planning to morphological changes in the patient's brain. There are already several studies that have evaluated the techniques and feasibility of cortical sparing, which show a significant dose reduction to the cortex, while maintaining adequate target coverage. ${ }^{82-84}$ Should a clear indication arise that radiotherapy delivered to certain cortical regions causes cognitive decline, a decision needs to made whether to favour sparing of the hippocampus, or sparing of the cerebral cortex. A controlled trial comparing the effect of cortical sparing versus hippocampal sparing on cognitive outcome may provide an answer to this question.

\section{CONCLUSION}

Thinning of the cerebral cortex is associated with cognitive decline. Thickness and volume of the cerebral cortex can be easily measured from TI MRI's that are acquired as part of the normal clinical routine. There seems to be a relation between radiation therapy and changes in cortical thickness and volume. Furthermore, higher doses may lead to more thinning and sharper cognitive decline. However, despite the amount of studies found, the present studies are too inhomogeneous and lacking in quality to safely make this conclusion or make suggestions for changes in clinical practice. Therefore, we recommend that multiple larger, longitudinal studies need to be performed that addresses the methodological problems stated in this review, in order to identify vulnerable cortical areas. If such areas do indeed exist, we may avoid them during radiation therapy, the same way we now are able to do for the hippocampus. Newest radiotherapy planning software and linear accelerator hardware would enable precise sparing of these cortical structures. With this, we can treat brain tumours optimally while preserving quality of life of our patients after radiotherapy.

\section{ACKNOWLEDGEMENTS}

Special thanks to Felix Weijdema MSc (Subject Librarian Medicine, Utrecht University, Utrecht, the Netherlands) for helping set up and conducting the search. 


\section{REFERENCES}

I. Makale MT, McDonald CR, Hattangadi-Gluth JA, Kesari S. Mechanisms of radiotherapy-associated cognitive disability in patients with brain tumours. Nature Reviews Neurology. 2017;13(I):52-64. doi:10.1038/nrneurol.2016.185

2. Greene-Schloesser D, Robbins ME. Radiation-induced cognitive impairment-from bench to bedside. Neuro-Oncology. 2012;14(SUPPL.4):iv37-iv44. doi:I0.1093/neuonc/nos196

3. Lee YW, Cho HJ, Lee WH, Sonntag WE. Whole brain radiation-induced cognitive impairment: pathophysiological mechanisms and therapeutic targets. Biomolecules \& therapeutics. 20I2;20(4):357370. doi:I0.4062/biomolther.2012.20.4.357

4. Greene-Schloesser D, Robbins ME. Radiation-induced cognitive impairment-from bench to bedside. Neuro-Oncology. 2012;14(SUPPL.4):iv37-iv44. doi:I0.1093/neuonc/nos196

5. Yin C, Li S, Zhao W, Feng J. Brain imaging of mild cognitive impairment and Alzheimer's disease. Neural regeneration research. 2013;8(5):435-444. doi:I0.3969/j.issn.1673-5374.2013.05.007

6. Pääkkö E, Harila-Saari A, Vanionpää L, Himanen S, Pyhtinen J, Lanning M. White matter changes on MRI during treatment in children with acute lymphoblastic leukemia: correlation with neuropsychological findings. Medical and pediatric oncology. 2000;35(5):456-46I. doi:10.1002/10969l|X(2000II0I)35:5<456::AID-MPO3>3.0.CO;2-I

7. Fujii $O$, Tsujino K, Soejima T, Yoden E, Ichimiya Y, Sugimura K. White matter changes on magnetic resonance imaging following whole-brain radiotherapy for brain metastases. Radiation Medicine. 2006;24(5):345-350. doi:10.1007/s |1604-006-0039-9

8. Gondi V, Hermann BP, Mehta MP, Tome WA. Hippocampal dosimetry predicts neurocognitive function impairment after fractionated stereotactic radiotherapy for benign or low-grade adult brain tumors. International journal of radiation oncology, biology, physics. 2013;83(4):e487-93. doi:10.1016/j. ijrobp.2011.10.021

9. Gondi V, Tomé WA, Mehta MP. Why avoid the hippocampus? A comprehensive review. Radiotherapy and Oncology. 2010;97(3):370-376. doi:10.1016/j.radonc.2010.09.013

10. Fischl B, Dale AM. Measuring the thickness of the human cerebral cortex from magnetic resonance images. Proceedings of the National Academy of Sciences of the United States of America. 2000;97(20):II050-II055. doi:I0.1073/pnas.200033797

II. Kandel ER, Schwartz JH, eds. Principles of Neural Science, Fifth Edition. New York: McGraw-Hill; 2013.

12. Mancall EL, Brock DG, eds. Gray's Clinical Neuroanatomy. Philadelphia: Elsevier Saunders; 2010.

13. Bosma I, Douw L, Bartolomei F, et al. Synchronized brain activity and neurocognitive function in patients with low-grade glioma: a magnetoencephalography study. Neuro-oncology. 2008;10(5):734744. doi:10.1215/15228517-2008-034

14. Valk PE, Dillon WP. Radiation injury of the brain. AJNR American journal of neuroradiology. 1991;12(I):4562.

15. Postma TJ, Klein M, Verstappen CCP, et al. Radiotherapy-induced cerebral abnormalities in patients with low-grade glioma. Neurology. 2002;59(I):I21-123.

16. Rabin BM, Meyer JR, Berlin JW, et al. Radiation-induced changes in the central nervous system and head and neck. Radiographics: a review publication of the Radiological Society of North America, Inc. 1996;16(5):1055-1072. doi:10.1148/radiographics.16.5.8888390

17. Harder $\mathrm{H}$, Holtel H, Bromberg JEC, et al. Cognitive status and quality of life after treatment for primary CNS lymphoma. Neurology. 2004;62(4):544-547.

18. Dahnke R, Yotter RA, Gaser C. Cortical thickness and central surface estimation. Neurolmage. 2013;65:336-348. doi:10.1016/j.neuroimage.2012.09.050

19. Kriegeskorte N, Goebel R. An Efficient Algorithm for Topologically Correct Segmentation of the Cortical Sheet in Anatomical MR Volumes. Neurolmage. 2001;14(2):329-346. doi:10.1006/ nimg.2001.0831

20. Ashburner J, Friston KJ. Voxel-Based Morphometry_The Methods. Neurolmage. 2000; I (6):805-82I . doi:I0.1006/nimg.2000.0582

21. Ashburner J, Friston KJ. Unified segmentation. Neurolmage. 2005;26(3):839-85I. doi:10.1016/j. neuroimage.2005.02.018 
22. Van Essen DC, Drury HA, Dickson J, Harwell J, Hanlon D, Anderson CH. An integrated software suite for surface-based analyses of cerebral cortex. Journal of the American Medical Informatics Association: JAMIA. 8(5):443-459.

23. June SK, Singh V, Jun KL, et al. Automated 3-D extraction and evaluation of the inner and outer cortical surfaces using a Laplacian map and partial volume effect classification. Neurolmage. 2005;27(I):210221. doi:10.1016/j.neuroimage.2005.03.036

24. MacDonald D, Kabani N, Avis D, Evans AC. Automated 3-D Extraction of Inner and Outer Surfaces of Cerebral Cortex from MRI. Neurolmage. 2000;12(3):340-356. doi:I 0.1006/nimg.1999.0534

25. Dahnke R, Gaser C. Surface and Shape Analysis. In: ; 20I8:5I-73. doi:I0.1007/978-I-4939-7647-8_4

26. Salat DH. Thinning of the Cerebral Cortex in Aging. Cerebral Cortex. 2004;14(7):721-730. doi:I0. I093/ cercor/bhh032

27. Du A-T, Schuff N, Kramer JH, et al. Different regional patterns of cortical thinning in Alzheimer's disease and frontotemporal dementia. Brain. 2007;130(4):II59-1166. doi:10.1093/brain/awm016

28. Pereira JB, Ibarretxe-Bilbao N, Marti M-J, et al. Assessment of cortical degeneration in patients with Parkinson's disease by voxel-based morphometry, cortical folding, and cortical thickness. Human Brain Mapping. 2012;33(II):2521-2534. doi:10.1002/hbm.21378

29. Lim HK, Jung WS, Ahn KJ, et al. Regional cortical thickness and subcortical volume changes are associated with cognitive impairments in the drug-naive patients with late-onset depression. Neuropsychopharmacology. 2012;37(3):838-849. doi:10.1038/npp.2011.264

30. Peterson BS, Warner V, Bansal R, et al. Cortical thinning in persons at increased familial risk for major depression. Proceedings of the National Academy of Sciences. 2009;106(I5):6273-6278. doi:10.1073/ pnas.08053III06

31. Seo SW, Im K, Lee J-M, et al. Cortical thickness in single- versus multiple-domain amnestic mild cognitive impairment. Neurolmage. 2007;36(2):289-297. doi:I0. I0I6/j.neuroimage.2007.02.042

32. Garcia-Diaz Al, Segura B, Baggio HC, et al. Cortical thinning correlates of changes in visuospatial and visuoperceptual performance in Parkinson's disease: A 4-year follow-up. Parkinsonism \& Related Disorders. 2018;46:62-68. doi:10.1016/j.parkreldis.2017.11.003

33. Gazdzinski LM, Cormier K, Lu FG, et al. Radiation-induced alterations in mouse brain development characterized by magnetic resonance imaging. International Journal of Radiation Oncology Biology Physics. 2012;84(5):e631-e638. doi:10.1016/j.jijrobp.2012.06.053

34. Selemon LD, Ceritoglu C, Ratnanather JT, et al. Distinct abnormalities of the primate prefrontal cortex caused by ionizing radiation in early or midgestation. The Journal of comparative neurology. 2013;52I(5):1040-1053. doi:10. I002/cne.23217

35. Aldridge K, Wang L, Harms MP, et al. A longitudinal analysis of regional brain volumes in macaques exposed to x-irradiation in early gestation. PLoS ONE. 2012;7(8):e43109. doi:I0.1371/journal. pone.0043109

36. Winkler AM, Kochunov P, Blangero J, et al. Cortical thickness or grey matter volume? The importance of selecting the phenotype for imaging genetics studies. Neurolmage. 2010;53(3):1135-1/46. doi:10.1016/j.neuroimage.2009.12.028

37. Moher D, Liberati A, Tetzlaff J, Altman DG. Preferred Reporting Items for Systematic Reviews and Meta-Analyses: The PRISMA Statement. PLoS Medicine. 2009;6(7):el000097. doi:10.1371/journal. pmed. I000097

38. Knoll T, Omar MI, Maclennan S, et al. Key Steps in Conducting Systematic Reviews for Underpinning Clinical Practice Guidelines: Methodology of the European Association of Urology. European urology. 2018;73(2):290-300. doi:10.1016/j.eururo.2017.08.016

39. Karunamuni R, Bartsch H, White NS, et al. Dose-Dependent Cortical Thinning After Partial Brain Irradiation in High-Grade Glioma. International journal of radiation oncology, biology, physics. 2016;94(2):297-304. doi:10.1016/j.ijrobp.2015.10.026

40. Seibert TM, Karunamuni R, Kaifi S, et al. Cerebral Cortex Regions Selectively Vulnerable to Radiation Dose-Dependent Atrophy. International Journal of Radiation Oncology Biology Physics. 2017;97(5):9109|8. doi:10.1016/j.jjrobp.2017.01.005 
4I. Correa DD, Root JC, Baser R, et al. A prospective evaluation of changes in brain structure and cognitive functions in adult stem cell transplant recipients. Brain Imaging and Behavior. 2013;7(4):478490. doi:|0.1007/s||682-013-9221-8

42. Kundu P, Li MD, Durkee BY, et al. Chemoradiation impairs normal developmental cortical thinning in medulloblastoma. Journal of neuro-oncology. 2017;133(2):429-434. doi:I0. I007/s I 1060-017-2453-5

43. Lin J, Lv X, Niu M, et al. Radiation-induced abnormal cortical thickness in patients with nasopharyngeal carcinoma after radiotherapy. Neurolmage: Clinical. 2017;14:610-621. doi:10.1016/j.nicl.2017.02.025

44. Tamnes CK, Zeller B, Amlien IK, et al. Cortical surface area and thickness in adult survivors of pediatric acute lymphoblastic leukemia. Pediatric blood \& cancer. 2015;62(6): I027-I034. doi: I0.1002/pbc.25386

45. Liu AK, Marcus KJ, Fischl B, et al. Changes in Cerebral Cortex of Children Treated for Medulloblastoma. International Journal of Radiation Oncology Biology Physics. 2007;68(4):992-998. doi:10.1016/j. ijrobp.2007.01.034

46. Nieman BJ, Elizabeth De Guzman A, Gazdzinski LM, et al. White and gray matter abnormalities after cranial radiation in children and mice. International Journal of Radiation Oncology Biology Physics. 2015;93(4):882-891. doi:10.1016/j.jirobp.2015.07.2293

47. Simo M, Vaquero L, Ripolles P, et al. Longitudinal brain changes associated with prophylactic cranial irradiation in lung cancer. Journal of thoracic oncology : official publication of the International Association for the Study of Lung Cancer. 2016; I (4):475-486. doi:10.1016/j.jtho.2015.12.110

48. Simó M, Root JC, Vaquero L, et al. Cognitive and Brain Structural Changes in a Lung Cancer Population. Journal of Thoracic Oncology. 20I5;I0(I):38-45. doi:I0. I097/JTO.0000000000000345

49. Petr J, Platzek I, Hofheinz F, et al. Photon vs. proton radiochemotherapy: Effects on brain tissue volume and perfusion. Radiotherapy and oncology : journal of the European Society for Therapeutic Radiology and Oncology. Published online January 19, 2018. doi:10.1016/j.radonc.2017.11.033

50. Prust MJ, Jafari-Khouzani K, Kalpathy-Cramer J, et al. Standard chemoradiation for glioblastoma results in progressive brain volume loss. Neurology. 2015;85(8):683-69I. doi:I0.1212/WNL.000000000000I86I

51. Gommlich A, Raschke F, Wahl H, Troost EGC. Retrospective assessment of MRI-based volumetric changes of normal tissues in glioma patients following radio(chemo)therapy. Clinical and Translational Radiation Oncology. 2018;8:17-21. doi:10.1016/j.ctro.2017.11.008

52. Lv X-F, Zheng X-L, Zhang W-D, et al. Radiation-induced changes in normal-appearing gray matter in patients with nasopharyngeal carcinoma: a magnetic resonance imaging voxel-based morphometry study. Neuroradiology. 2014;56(5):423-430. doi:I0.1007/s00234-014-1338-y

53. Leng $X$, Fang $P$, Lin H, et al. Structural MRI research in patients with nasopharyngeal carcinoma following radiotherapy: A DTI and VBM study. Oncology letters. 2017;14(5):6091-6096. doi:10.3892/ ol.2017.6968

54. Hu F, Li T, Wang Z, et al. Use of 3D-ASL and VBM to analyze abnormal changes in brain perfusion and gray areas in nasopharyngeal carcinoma patients undergoing radiotherapy. Biomedical Research (India). 2017;28(18):7879-7885.

55. Edelmann MN, Krull KR, Liu W, et al. Diffusion tensor imaging and neurocognition in survivors of childhood acute lymphoblastic leukaemia. Brain. 2014;137(II):2973-2983. doi:10.1093/brain/awu230

56. Follin C, Erfurth EM, Johansson A, et al. Impaired brain metabolism and neurocognitive function in childhood leukemia survivors despite complete hormone supplementation in adulthood. Psychoneuroendocrinology. 2016;73:157-165. doi:10.1016/j.psyneuen.2016.07.222

57. Zeller B, Tamnes CK, Kanellopoulos A, et al. Reduced neuroanatomic volumes in long-term survivors of childhood acute lymphoblastic leukemia. Journal of Clinical Oncology. 2013;31(17):2078-2085. doi:10.1200/JCO.2012.47.4031

58. Porto L, Preibisch C, Hattingen E, et al. Voxel-based morphometry and diffusion-tensor MR imaging of the brain in long-term survivors of childhood leukemia. European radiology. 2008;|8(II):269|-2700. doi: 10.1007/s00330-008-1038-2

59. Reddick WE, Mulhern RK, Elkin TD, et al. A hybrid neural network analysis of subtle brain volume differences in children surviving brain tumors. Magnetic Resonance Imaging. 1998;16(4):413-421. doi:10.1016/S0730-725X(98)00014-9 
60. Riggs L, Bouffet E, Laughlin S, et al. Changes to memory structures in children treated for posterior fossa tumors. Journal of the International Neuropsychological Society. 2014;20(2):168-180. doi:10.1017/ S13556177/300I29X

61. Aldridge K, Wang L, Harms MP, et al. A longitudinal analysis of regional brain volumes in macaques exposed to x-irradiation in early gestation. PLOS ONE. 2012;7(8):e43109. doi:10.1371/journal. pone.0043109

62. Lumniczky K, Szatmári T, Sáfrány G. Ionizing Radiation-Induced Immune and Inflammatory Reactions in the Brain. Frontiers in immunology. 2017;8:517. doi:10.3389/fimmu.2017.00517

63. Fike JR, Cann CE, Turowski K, et al. Radiation dose response of normal brain. International Journal of Radiation Oncology*Biology*Physics. 1988;14(I):63-70. doi:10.1016/0360-3016(88)90052-I

64. Wong CS, Van der Kogel AJ. Mechanisms of radiation injury to the central nervous system: implications for neuroprotection. Molecular interventions. 2004:4(5):273-284. doi:1 0.II24/mi.4.5.7

65. Mancall EL, Brock DG, eds. Gray's Clinical Neuroanatomy. Philadelphia: Elsevier Saunders; 2010.

66. Yang L, Yang J, Li G, et al. Pathophysiological Responses in Rat and Mouse Models of Radiation-Induced Brain Injury. Molecular neurobiology. 20 17;54(2):1022-1032. doi:10.1007/s12035-015-9628-x

67. Lumniczky K, Szatmári T, Sáfrány G. Ionizing Radiation-Induced Immune and Inflammatory Reactions in the Brain. Frontiers in immunology. 2017;8:517. doi:10.3389/fimmu.2017.00517

68. Tillema J-M, Pirko I. Neuroradiological evaluation of demyelinating disease. Therapeutic advances in neurological disorders. 2013;6(4):249-268. doi:10.1 I77/1756285613478870

69. Benjamini Y, Hochberg Y. Controlling The False Discovery Rate - A Practical And Powerful Approach To Multiple Testing. Vol 57;; 1995. doi:10.2307/2346I0I

70. Nichols T, Hayasaka S. Controlling the familywise error rate in functional neuroimaging: a comparative review. Statistical methods in medical research. 2003;12(5):419-446. doi:I0.I191/0962280203sm34Ira

7l. Walhovd KB, Fjell AM, Giedd J, Dale AM, Brown TT. Through Thick and Thin: a Need to Reconcile Contradictory Results on Trajectories in Human Cortical Development. Cerebral Cortex. Published online January II, 2016:bhv30I. doi:I0.1093/cercor/bhv30I

72. Good CD, Johnsrude I, Ashburner J, Henson RN, Friston KJ, Frackowiak RS. Cerebral asymmetry and the effects of sex and handedness on brain structure: a voxel-based morphometric analysis of 465 normal adult human brains. Neurolmage. 2001;14(3):685-700. doi:I0.1006/nimg.2001.0857

73. Hamilton LS, Narr KL, Luders E, et al. Asymmetries of cortical thickness: effects of handedness, sex, and schizophrenia. Neuroreport. 2007;18(14):1427-1431. doi:10.1097/WNR.0b013e3282e9a5a2

74. Fjell AM, Grydeland H, Krogsrud SK, et al. Development and aging of cortical thickness correspond to genetic organization patterns. Proceedings of the National Academy of Sciences of the United States of America. 2015; II2(50):15462-15467. doi:10.1073/pnas. 150883।II2

75. Piccolo LR, Merz EC, He X, Sowell ER, Noble KG, Pediatric Imaging, Neurocognition GS. Age-Related Differences in Cortical Thickness Vary by Socioeconomic Status. PloS one. 2016; II(9):e01625II. doi:I0.1371/journal.pone.01625II

76. Hoekzema E, Barba-Müller E, Pozzobon C, et al. Pregnancy leads to long-lasting changes in human brain structure. Nature neuroscience. 2017;20(2):287-296. doi:10.1038/nn.4458

77. McDonald BC, Conroy SK, Ahles TA, West JD, Saykin AJ. Gray matter reduction associated with systemic chemotherapy for breast cancer: A prospective MRI study. Breast Cancer Research and Treatment. 2010;123(3):819-828. doi:I0.1007/sI0549-0I0-1088-4

78. Stouten-Kemperman MM, de Ruiter MB, Koppelmans V, Boogerd W, Reneman L, Schagen SB Neurotoxicity in breast cancer survivors $\geq 10$ years post-treatment is dependent on treatment type. Brain imaging and behavior. 2015;9(2):275-284. doi: I0.1007/s I 1682-014-9305-0

79. Rosenthal R. The file drawer problem and tolerance for null results. Psychological Bulletin. 1979;86(3):638-64l. doi:10.1037/0033-2909.86.3.638

80. Gondi V, Tolakanahalli R, Mehta MP, et al. Hippocampal-sparing whole-brain radiotherapy: a "how-to" technique using helical tomotherapy and linear accelerator-based intensity-modulated radiotherapy. International journal of radiation oncology, biology, physics. 2010;78(4):I244-1252. doi:10.1016/j. ijrobp.2010.01.039

81. Lagendijk JJW, Raaymakers BW, Raaijmakers AJE, et al. MRI/linac integration. Radiotherapy and Oncology. 2008;86(I):25-29. doi:10.1016/j.radonc.2007.10.034 
82. Exeli A-K, Kellner D, Exeli L, et al. Cerebral cortex dose sparing for glioblastoma patients: IMRT versus robust treatment planning. Radiation oncology (London, England). 2018;13(I):20. doi:I0.1I86/ sl3014-018-0953-x

83. Karunamuni RA, Moore KL, Seibert TM, et al. Radiation sparing of cerebral cortex in brain tumor patients using quantitative neuroimaging. Radiotherapy and oncology : journal of the European Society for Therapeutic Radiology and Oncology. 2016;118(1):29-34. doi:10.1016/j.radonc.2016.01.003

84. Murzin VL, Woods K, Moiseenko V, et al. 4pi plan optimization for cortical-sparing brain radiotherapy. Radiotherapy and oncology: journal of the European Society for Therapeutic Radiology and Oncology. Published online March 2018. doi:10.1016/j.radonc.2018.02.011

85. Desikan RS, Ségonne F, Fischl B, et al. An automated labeling system for subdividing the human cerebral cortex on MRI scans into gyral based regions of interest. Neurolmage. 2006;3I (3):968-980. doi:10.1016/J.NEUROIMAGE.2006.01.021

86. Gaser C, Dahnke R. CAT - A Computational Anatomy Toolbox for the Analysis of Structural MRI Data. HBM Conference 2012. 2012;32(7):7743. 


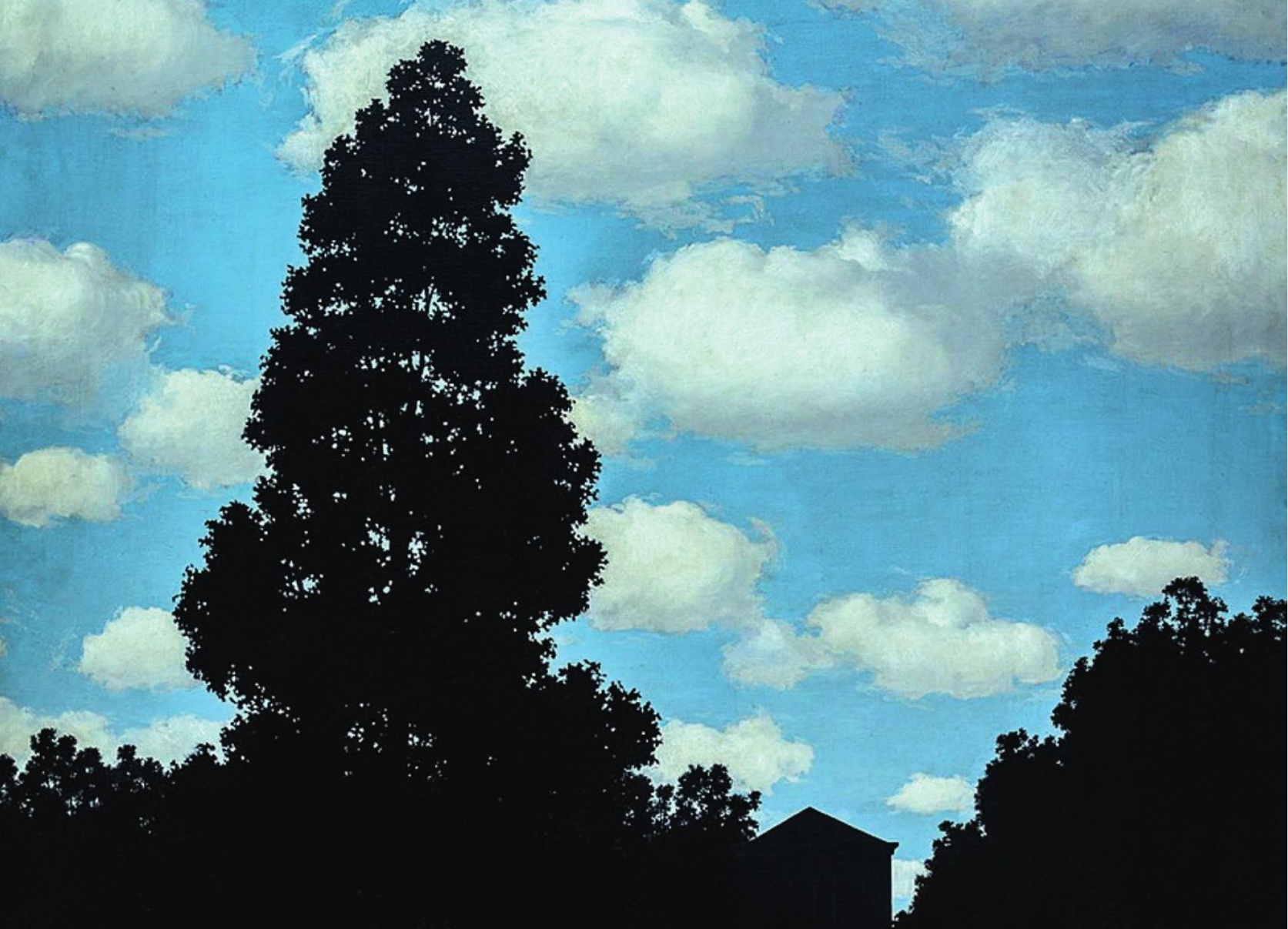

4t

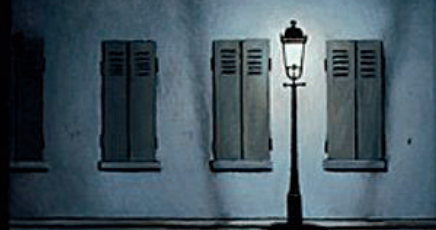




\section{Chapter 3}

Effect of radiation therapy on cerebral cortical thickness in glioma patients: treatment-induced thinning of the healthy cortex

S.H.J. Nagtegaal, S. David, T.J. Snijders, M.E.P. Philippens, A. Leemans, J.J.C. Verhoeff 


\section{ABSTRACT}

\section{Background}

Radiation-induced brain injury is an increasing issue in the treatment of brain tumors now overall survival is improving. One of the effects of radiation therapy (RT) is thinning of the cerebral cortex, which could be one of the factors contributing to cognitive impairments after treatment. In healthy brain, cortex thickness varies between I and $4.5 \mathrm{~mm}$. In this study, we assess the effect of RT on the thickness of the cerebral cortex, and relate the changes to the local dose.

\section{Methods}

We identified 28 glioma patients with optimal scan quality. Clinical CTs and MRIs at baseline and I year post-RT were collected and coregistered. The scans were processed via an automated image processing pipeline, which enabled measuring changes of the cortical thickness, which were related to local dose.

\section{Results}

Three areas were identified where significant dose-dependent thinning occurred, with thinning rates of 5, 6 and $26 \mu \mathrm{m} / \mathrm{Gy}$ after I year, which corresponds to losses of 5.4\%, 7.2\% and $21.6 \%$ per 30 Gy per year. The first area was largely located in the right inferior parietal, supramarginal and superior parietal regions, the second in the right posterior cingulate and paracentral regions, and the third almost completely in the right lateral orbital frontal region.

\section{Conclusions}

We have identified three areas susceptible to dose-dependent cortical thinning after radiation therapy. Should future prospective studies conclude that irradiation of these areas leads to cognitive decline, they need to be spared in order to prevent this debilitating consequence of treatment. 


\section{INTRODUCTION}

Radiation therapy (RT) plays an important part in the treatment of brain malignancies, both for primary and metastatic disease. It comes, however, with the unfortunate consequence of radiation-induced brain injury. One of the major and most debilitating symptoms of this phenomenon is progressive cognitive decline, which occurs in in 50-90\% of patients undergoing RT to the brain. ${ }^{1,2}$ It most likely has a multifactorial origin, with roles for vascular damage, demyelination and neuronal dysfunction.,3,4 Morphological changes in the hippocampus, white matter and cerebral cortex related to radiation-induced brain injury can be seen on routine imaging ${ }^{5-9}$ (see Figure I), and specialized software allows for accurate measurement of these changes. ${ }^{10-13}$ Interest in the effect of RT on cortical thickness and volume has increased in the recent years, but high-quality evidence remains sparse. In a recent review ${ }^{14}$ the current knowledge was assessed, and two papers showing cortical thinning after RT were found. Karunamuni et al. was the first to show dose-dependent cortical thinning after RT in 15 glioma patients. ${ }^{15}$ Seibert et al. went on to examine brain regions associated with cognitive function, and found these to be susceptible to radiationinduced damage. ${ }^{16}$

Changes in cortical thickness have been linked to cognitive decline, suggesting thinning of the cortex has a role in the etiology of radiation-induced cognitive impairments. ${ }^{17,18}$ Therefore, it would be of interest to assess whether RT does indeed cause thinning of the cortex, and if this is related to the administered dose. Identification of areas susceptible to radiationinduced damage may encourage sparing of these areas.

In this study, we wish to add to the existing evidence, by assessing the effect of radiation therapy on the thickness of the cerebral cortex, and relating the changes to the local dose. 


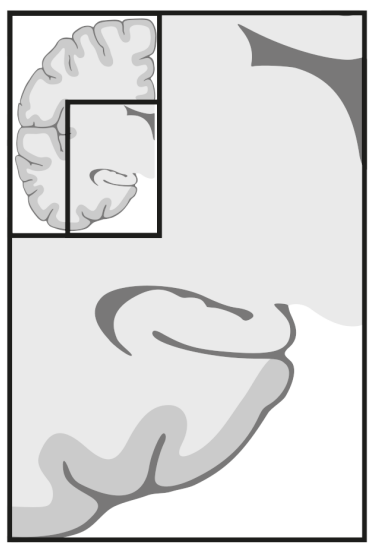

Before

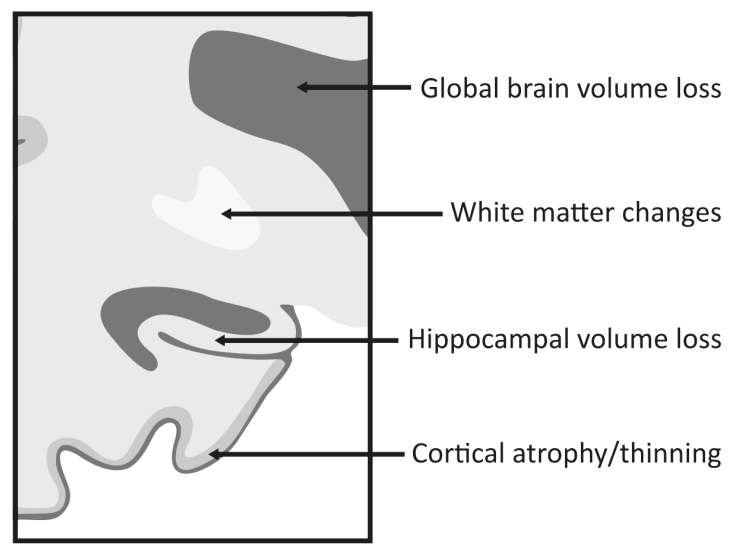

After

Figure I Previously reported radiological changes in the post-RT brain related to cognitive changes

\section{METHODS}

\section{Patient selection and data collection}

We retrospectively identified patients who were treated with RT for newly discovered grade II-IV glioma at the department of Radiation Oncology of our institution in 2016 and 2017. Patients were eligible for inclusion when the following criteria were met: treatment planning CT and MRI present, and of sufficient resolution; survival of at least 270 days after RT; at least I follow-up MRI between 270 days and 360 days after RT present, and of sufficient resolution. Clinical MRI and CT scans made for RT treatment planning were extracted from patient records, along with all follow-up MRIs, and clinical and demographic characteristics. Informed consent for this retrospective study was waived by our institutional review board (\#|8/274).

\section{Image acquisition}

For every patient the pre-RT CT and MRI were collected, as well as all available follow-up MRIs. MR images were acquired on a $3 T$ Philips Ingenia scanner (Philips Medical Systems, Best, The Netherlands) as part of routine clinical care. TI-weighted MR images were acquired with a 3D turbo-spin echo (TSE) sequence without gadolinium enhancement with the following parameters: TR $=8.1 \mathrm{~ms}, \mathrm{TE}=3.7 \mathrm{~ms}$, flip angle $=8^{\circ}, 213$ continuous axial slices without gap, matrix: $207 \times 289$, voxel resolution I $\times 0.96 \times 0.96 \mathrm{~mm}^{3}$. The planning CT scans were acquired on a Brilliance Big bore scanner (Philips Medical Systems, Best, The Netherlands), with a tube potential of $120 \mathrm{kVp}$, with use of a matrix size of $512 \times 512$ and $0.65 \times 0.65 \times 3.0 \mathrm{~mm}$ voxel size. 


\section{Image processing}

All imaging data was processed with Statistical Parametric Mapping (SPM), ${ }^{19}$ Computational Anatomy Toolbox (CATI2) $)^{20}$ and in-house algorithms developed in MATLAB (Mathworks, Natick, Massachusetts, United States). Image processing was done according to our own previously published criteria. ${ }^{14} \mathrm{~A}$ graphical overview of the image processing pipeline is shown in Figure 2. More detailed methods can be found in Appendix I.

In brief, the cropped CT image with the associated dose and planning target volume (PTV) maps was registered to the TI MR images. This step resulted in the CT image and the MRIs being in the same space. Next, the rigidly coregistered TIs were processed with CATI2's segmentation pipeline. If the residual damage to the tumor area (e.g. edema, surgical scarring, tumor bed) extended beyond the PTV in either the baseline or the follow-up images, then the affected subject was removed from the analysis to prevent tissue misclassification.

Cortical thickness and central surfaces were estimated with CATI2 by means of the fully automated projection-based thickness estimation method. Dose maps and PTV maps (dilated by $3 \mathrm{~mm}$ and smoothed) were mapped to the template surface, meaning that all individual features (cortical thickness, dose and PTV mask) were mapped to the same template surface. The within-subject difference in cortical thickness was calculated by subtracting the baseline and the follow-up cortical thickness surfaces in every vertex (a vertex being analogous to a voxel, but on a surface). In every subject the cortical thickness difference and the dose maps were censored with the surface resampled PTV maps to avoid spurious thickness-dose relations, which may originate from false thickness estimation around the tumor. This resulted in dose and cortical thickness change maps, in which the tumor bed and tumor scar was censored, and which could be used for further analysis.

\section{Statistical analysis}

Vertexwise statistical comparison of cortical thickness change and dose correlation was carried out with a permutation test with 10,000 iterations performed with the permutation analysis of linear models (PALM) toolbox in Matlab. ${ }^{21-23}$ Significance of a correlation was set at $\mathrm{P}_{\text {corr }}<0.05$, using family-wise error rate (FWER) adjustment to correct for multiple comparisons. Age at the time of the diagnosis and sex of the patients were included as nuisance regressors. Multiple effect size measures and the yearly rate of change per dose [in \%/Gy/year], are reported to grade the practical significance of the results. ${ }^{24-26}$ 

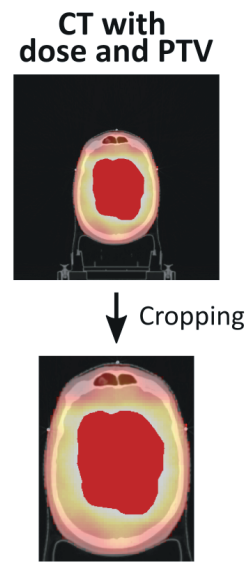

$\downarrow$ Rigid registration
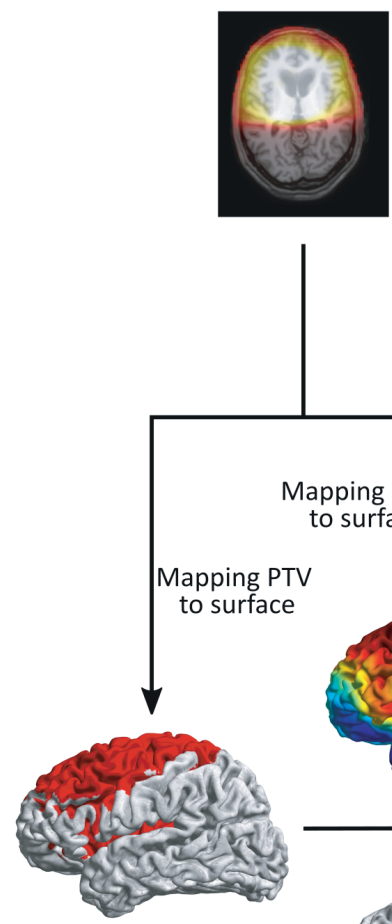

MRI before RT
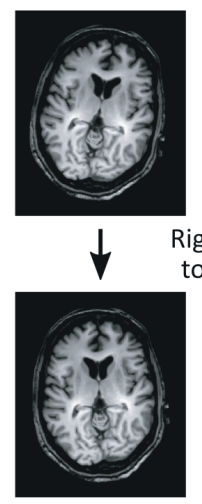

$\downarrow$

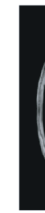

MRI after RT

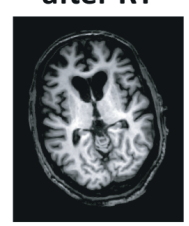

ion

Rigid registration
to the midpoint

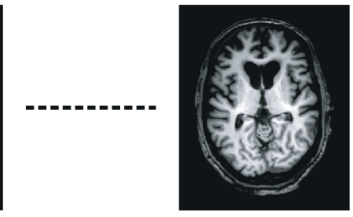

Preprocessing $\downarrow$

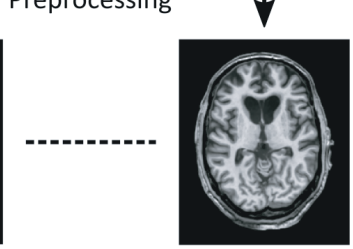

$\downarrow \begin{gathered}\text { Cortical thickness } \\ \text { estimation }\end{gathered}$
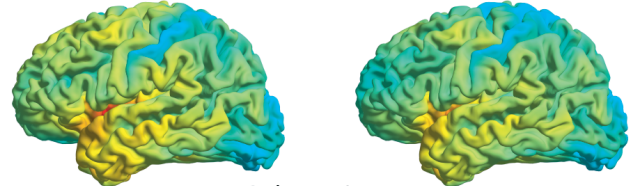

Subtracting cortical thickness

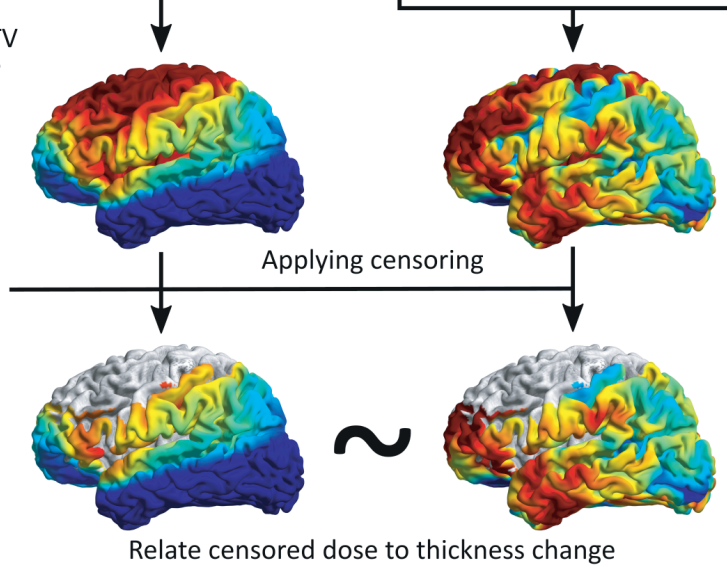

Figure 2 Image processing pipeline. PTV = planned target volume, RT = radiation therapy 
To test whether chemotherapy has an effect on the dose/thickness relation, a sensitivity analysis was performed in which we corrected for chemotherapy type (chemotherapy vs no chemotherapy). By adding this variable to the nuisance regressions of the permutation test performed in the primary analysis, we assessed see whether this would lead to different results. Similar to the primary analysis, 10,000 iterations were performed with the PALM toolbox, using FWER adjustment to correct for multiple comparisons.

\section{RESULTS}

\section{Participants}

Of all patients who underwent RT for glioma between in 2016 and 201728 fulfilled our inclusion criteria and were selected for further analysis. A flow chart of study inclusion is shown in Supplementary Figure SI. Extensive cerebral edema after RT outside the PTV on the baseline imaging meant exclusion from further analysis in three patients. Baseline characteristics are shown in Table I.

Table I Baseline characteristics of included patients

\begin{tabular}{ll}
\hline & N (total $\mathbf{n}=\mathbf{2 8})$ \\
\hline Age (mean; SD) & $49( \pm 15)$ \\
Sex & $17(60.7 \%)$ \\
Male & $11(39.3 \%)$ \\
Female & \\
WHO grade & $11(39.3 \%)$ \\
II & $7(25 \%)$ \\
III & $10(35.7 \%)$ \\
IV & \\
Tumor type & $13(46.4 \%)$ \\
Astrocytoma & $3(10.7 \%)$ \\
Oligodendroglioma & $1(3.6 \%)$ \\
Mixed & $1(3.6 \%)$ \\
Ganglioglioma & $10(35.7 \%)$ \\
Glioblastoma & \\
Prescribed dose & $11(39.3 \%)$ \\
$28 \times 1.8=50.4$ Gy & $2(7.1 \%)$ \\
$30 \times 1.8=54$ Gy & $15(53.6 \%)$ \\
$30 \times 20=60$ Gy & \\
Chemotherapy & $4(14.3 \%)$ \\
None & $20(71.4 \%)$ \\
Temozolomide & $4(14.3 \%)$ \\
PCV &
\end{tabular}

$\mathrm{PCV}=$ procarbazine, lomustine and vincristine 


\section{Cortical thickness}

Mean cortical thickness at baseline and follow-up, as well as relative and absolute differences between the two are shown in Supplementary Figure S2. Maps of maximum, mean and minimum dose to the surface are shown in Supplementary Figure S3.

Three areas were identified where significant dose-dependent thinning occurred, with thinning rates of 5, 6 and $26 \mu \mathrm{m} / \mathrm{G}$. The first area was largely located in the right inferior parietal, supramarginal and superior parietal regions, the second in the right posterior cingulate and paracentral regions, and the third almost completely in the right lateral orbital frontal region. The precise location of the areas can be seen in Figure 3, and the details per region are shown in Table 2. A map showing the spatial distribution of censorings in the cortex is shown in Figure 4. A map of corrected p-values of the relationship between dose and cortical thinning is shown in Supplementary Figure S4.

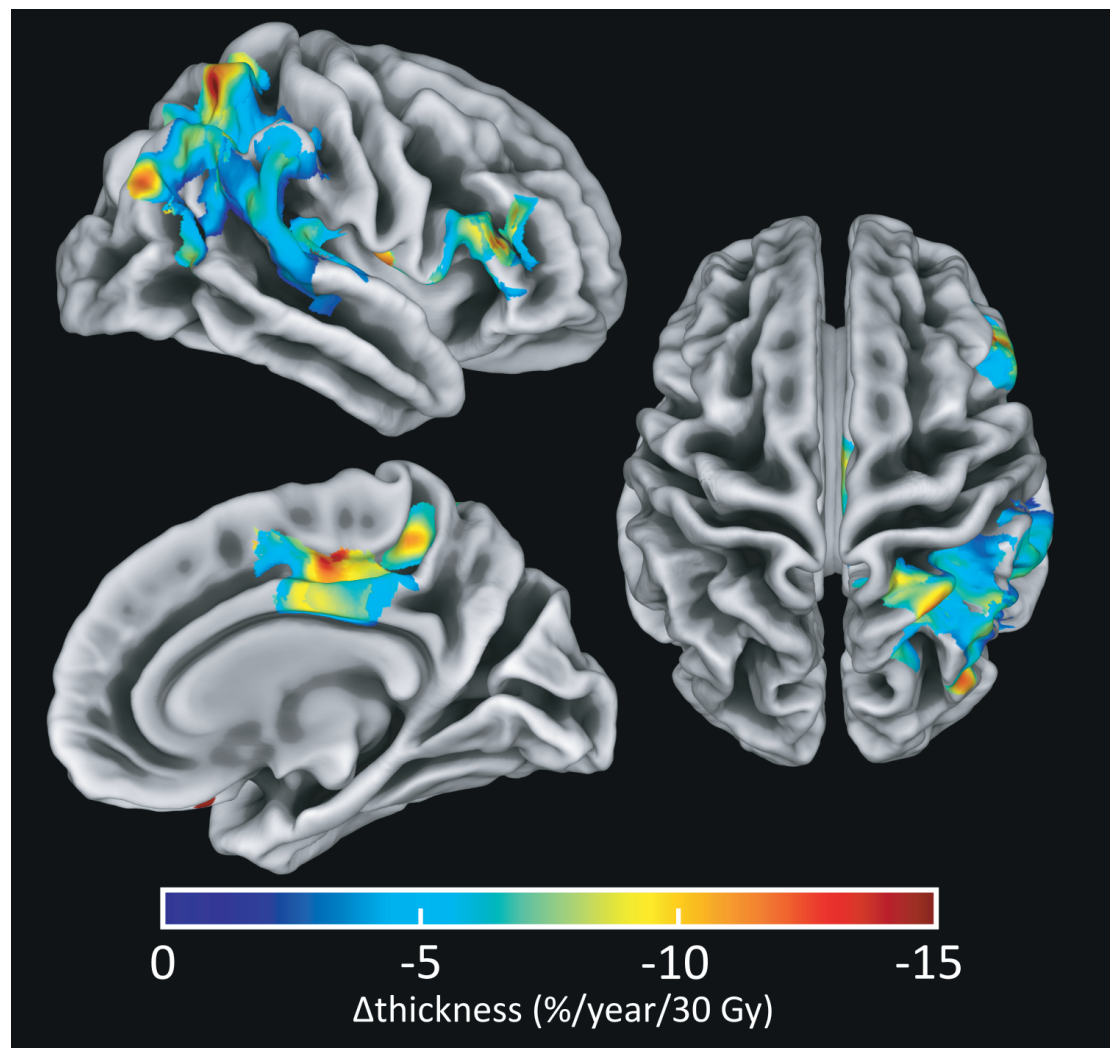

Figure 3 Areas of significant dose-dependent cortical thinning, in \% change/year /30 Gy 


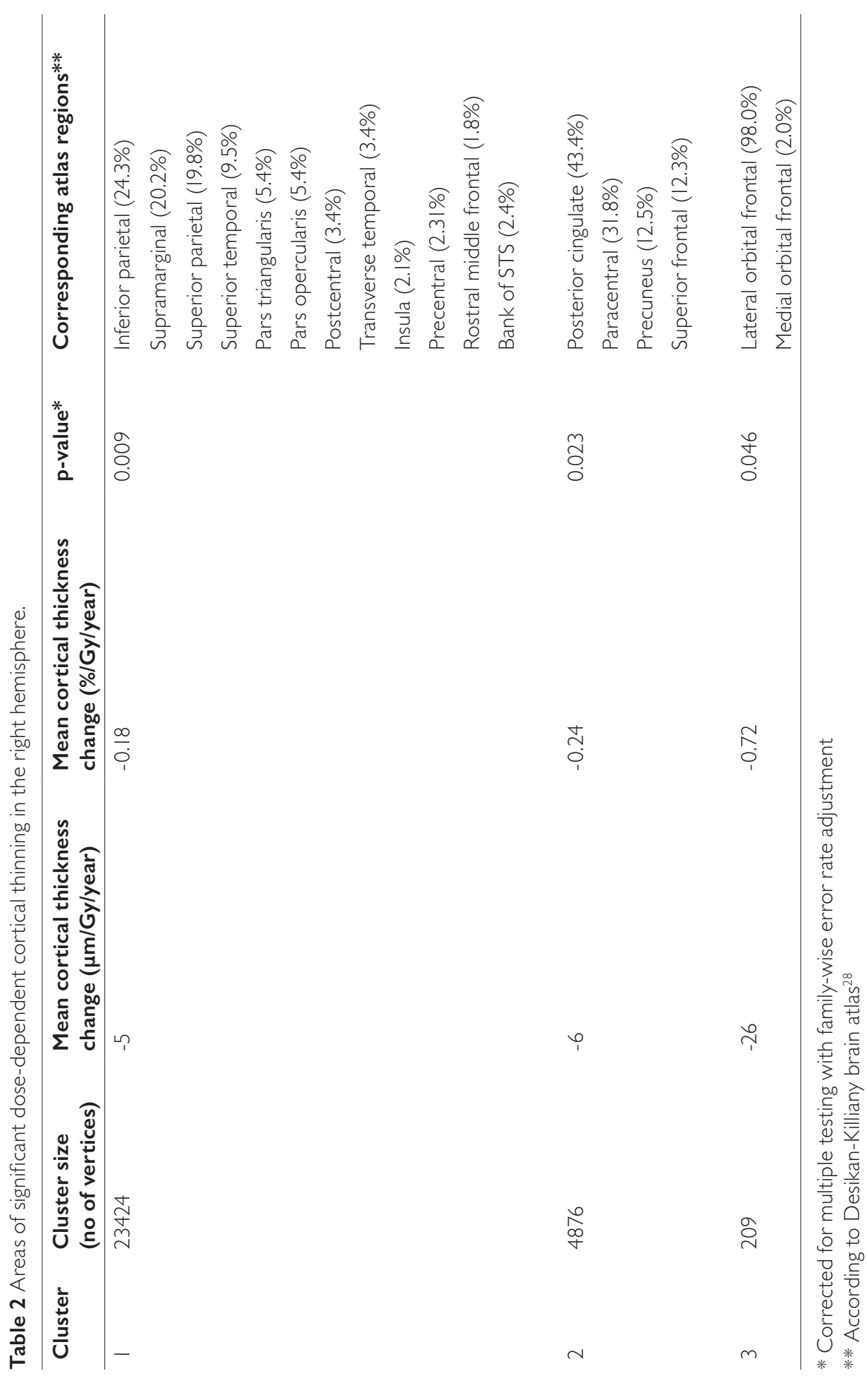


A sensitivity analysis was done to test whether chemotherapy has an additional effect on the dose/thickness relation. The results are shown in Supplementary Table I. The same areas with a significant result were found as in the primary analysis, with similar thinning rates and $p$-values. Only small variations in cluster size and corresponding atlas regions were present, resulting in region 2, as found in the primary analysis, now being identified as two separate regions with the same cortical thinning rate of $-6 \mu \mathrm{m} / \mathrm{G} y$.

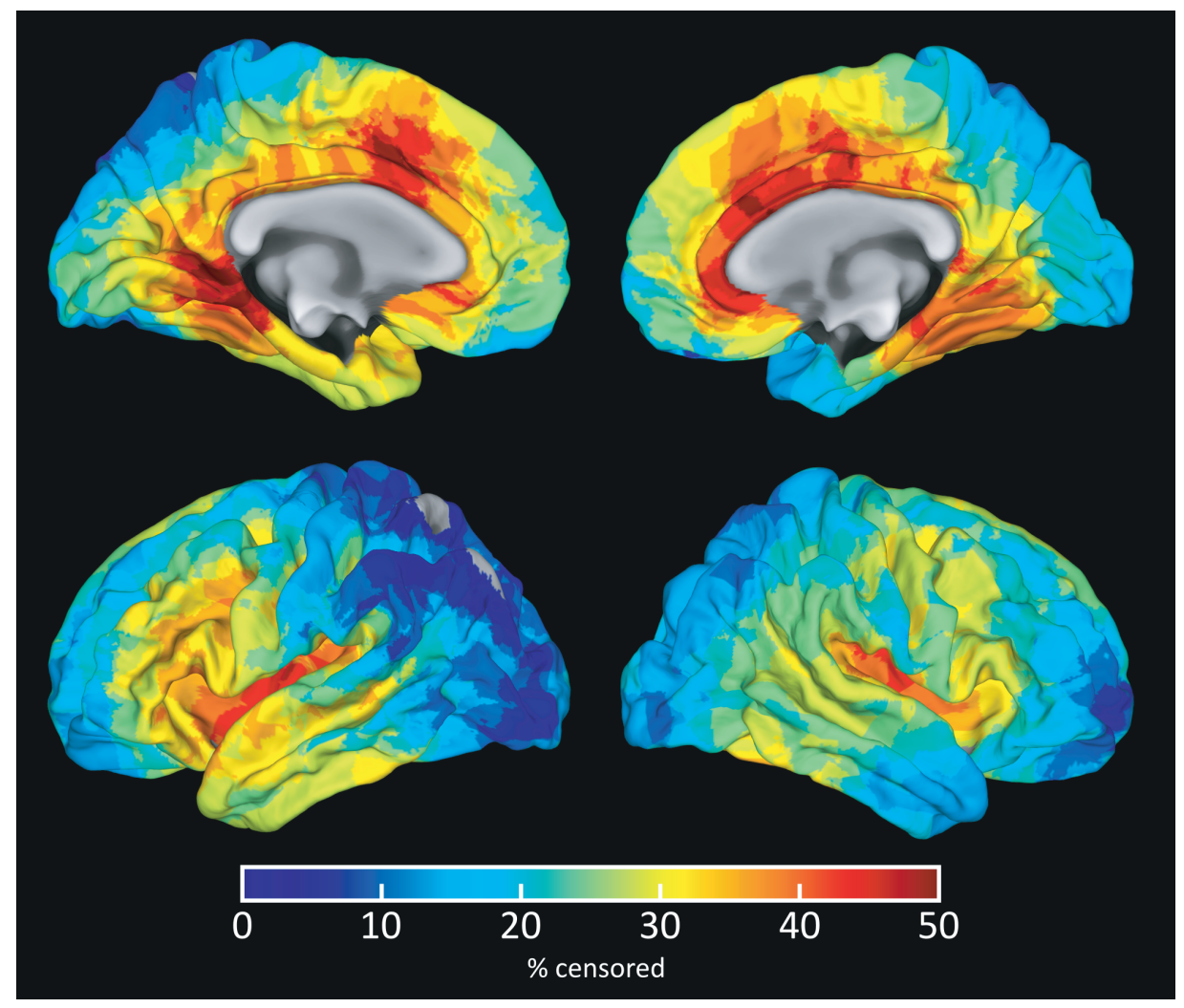

Figure 4 Heatmap showing the number of censored areas in the cortex

\section{DISCUSSION}

In this study, we examined the change in cortical thickness after radiation treatment (RT) of the brain. We found that there are three areas in the right hemisphere that show significant dose-dependent cortical thinning. The effect of RT on these cortical areas is in the same order of magnitude as aging by several years, in some regions even up to a decade. ${ }^{27,28}$ 
Cortical thickness and volume change have been examined before in several other studies with varying levels of evidence, ${ }^{14}$ with the most complete and robust analysis presented in the papers by Karunamuni et al. and Seibert et al. ${ }^{15,16}$ Karunamuni et al. analyzed 15 glioma patients before and after RT, and found dose-dependent cortical thinning, with rates of 1.3 $\mu \mathrm{m}$ (up to 34.6 Gy) and 7.2 $\mu \mathrm{m} / \mathrm{Gy}$ (above 34.6 Gy). Seibert et al found several brain regions (pre-specified according to the Desikan-Killiany brain atlas ${ }^{29}$ ) which showed a decrease in cortical thickness with increased dose. Two of these regions, the inferior parietal and superior temporal, overlap with the areas found in our analysis, and showed thinning rates from 2.3 and $4.4 \mu \mathrm{m} / \mathrm{Gy}$, respectively

The areas showing dose-dependent identified in the current work are comprised of several functional cortical areas involved in cognitive processes. ${ }^{30}$ The supramarginal, inferior parietal, and superior temporal gyri, the latter two also identified by Seibert et al. $^{16}$, are part of Wernicke's area. This area is involved in both language comprehension and speech production, and damage to it can lead to aphasia. ${ }^{31}$ The first cluster also contained the superior parietal lobule, which is an somatosensory association area, involved in visuospatial coordination and working memory. ${ }^{30,32}$ The posterior cingulate cortex is well connected, being part of both the hippocampal and limbic systems, and is associated with memory and emotion. ${ }^{30}$ Finally, the lateral orbital frontal gyrus is located in the ventromedial prefrontal cortex. Impulse control is in part mediated by this region, as a results of its connection to the amygdala. ${ }^{30}$

Loss of cortical thickness is part of the normal aging process. ${ }^{33}$ However, accelerated cortical thinning (with rates comparable to our findings) has also been observed in neurodegenerative diseases, such as Alzheimer's disease ${ }^{34,35}$ and Parkinson's disease, ${ }^{36}$ as well as other disorders such as depression. ${ }^{37,38}$ An association between cortical thinning and cognitive impairments has also been found, which suggests that cortical thinning is one of the mechanisms underlying the impairments observed after radiation therapy. ${ }^{17,18}$

The pathophysiological process that leads to a diminished cortex remains unknown. Observed differences in the post-RT brain include phagocytosis of healthy and damaged neurons though activated microglia, ${ }^{39}$ loss of glial cells, ${ }^{40}$ and demyelination through vascular damage. ${ }^{1,4,41}$ The exact mechanism behind cortical thinning is most likely a combination of some or all of these radiation effects.

As mentioned before, the changes in the cerebral cortex are not the only effects that are seen after RT (see Figure I). Deep grey matter structures including the hippocampus ${ }^{42}$ and the amygdala ${ }^{43}$ were already shown to have a susceptibility to dose-dependent volume loss 
after RT. These structures, as well as the cerebral cortex, may in the future be considered as organs at risk (OAR) to be avoided by the radiotherapy planning system.

Due to advances in therapy, mortality of cancer has decreased over the last decades, which means that the number of cancer survivors has increased; ${ }^{44}$ although gliomas can rarely be treated curatively, the therapeutic advances have led to improved overall survival for patients. Naturally, this is a desirable development, but it also means that long-term consequences of anti-cancer treatment have to be examined. If a treatment leads to excellent survival, but also comes with serious chronic adverse effects, the consideration has to be made whether this is the optimal treatment. Patient-tailored decisions need to be made, and patients need to be informed not only of the expected benefits of survival, but also of the long-term consequences. As the prominent long-term effect of brain tumor treatment is cognitive decline, we need to be able to predict what certain therapies do to patients' cognitive abilities.

In this work we established an association between cortical atrophy and dose, while the clinically significant relation between changes in cortical thickness and the changes in the patients' cognition remains unknown. Important to note is that neurophysiological investigations already revealed the connection between RT and cognition.1,2 The hypothesis that cortical thickness decrease relates to cognitive symptoms, just like in other brain diseases such as Alzheimer's disease, fits in this line of observations. As far as we know no study has examined the relation between these three factors in a single prospective cohort, which seems to be the reasonable follow-up to understand this debilitating phenomenon.

Additionally, the feasibility of cortical sparing should be examined, the possibility of which has already been shown. ${ }^{45,46}$ If areas of dose-dependent cortical thinning are found that affect the cognitive outcomes after RT, these areas need to receive the lowest possible dose while still maintaining adequate tumor coverage and sparing organs at risk. This can be achieved with techniques such as volumetric modulated arc therapy (VMAT) or proton therapy. The balance between treating the cancer and reducing the long-term effects of said treatment is something that needs to be explored in the future.

Limitations of this study include the limited number of scans suitable for analysis. As discussed in the results, only 46 out of a total of 206 glioma patients had scans of sufficient quality to perform proper and reliable cortical thickness measurements. While this rigorous selection ensures the best possible results, it does limit the power required to find small differences between the pre-RT and post-RT cortex. 
Secondly, the administration of chemotherapy to the majority of patients could have an effect on the cortical thickness. While the agents administered in this study have not been linked to changes in brain morphology, studies in breast cancer and childhood leukemia show that certain chemotherapeutic agents have an effect on both white and grey matter dimentions. ${ }^{47-50}$ However, our study examined the relation between dose and thickness, and any effects of chemotherapy on the cortex are expected to be independent of radiation dose, as well as region-independent. However, we cannot exclude the possibility that the observed differences are partly related to the administered chemotherapeutic agents. In a sensitivity analysis in which we corrected for chemotherapy, we found no major changes to the main results, suggesting the role of chemotherapy to the observed effects is limited.

Also, in fitting a linear model to the dose-thickness relation we may have overlooked any non-linear relation between the two factors. It is important to note, however, that the exact relation between dose and cortical thinning is still not well understood. Therefore unsupported, advanced models may result in spurious overfitting. With a robust and automated image processing pipeline as well as statistical inference, we ensured to exclude false positive results.

Another possibility in this study is the introduced selection bias, as we have selected patients with long enough survival, which may influence the results. However, we are interested in long-term effects of RT, which are of course only relevant to patients with long survival times. Another possible source of selection bias is the fact that we excluded patients with large cerebral damage beyond the PTV that could influence the automatic cortical thickness estimation. However, this occurred in only 3 patients, so we do not expect a big influence of this fact on the found results. This study also only examines glioma patients, which might mean our results are not applicable to other primary and metastatic brain tumors.

Furthermore, the proximity of areas receiving higher dose to the tumor means that regional effects of the tumor microenvironment could have influenced the results. We therefore cannot rule out a tumor effect independent from the effect of radiation. However, as the area receiving dose is larger than the area of the tumor, and as we have observed cortical thinning even at low doses, we expect this effect to not greatly influence our results.

Finally, the censoring pattern shown in Figure 4 could lead to overrepresentation of certain cortical regions in our dataset. As the doses around the censored area, and thus around the PTV, are highest, a dose-thickness relation is likely to be found in these regions. The censored areas are concentrated towards the frontal cortex, and two of our three identified regions are within areas of increased censoring. 
To conclude, we have identified three areas susceptible to dose-dependent cortical thinning after radiation therapy. Should future studies conclude that irradiation of these areas lead to cognitive decline, they need to be spared in order to prevent this debilitating consequence of treatment. 


\section{REFERENCES}

I. Makale MT, McDonald CR, Hattangadi-Gluth JA, Kesari S. Mechanisms of radiotherapy-associated cognitive disability in patients with brain tumours. Nature Reviews Neurology. 2017;13(I):52-64. doi:10.1038/nrneurol.2016.185

2. Greene-Schloesser D, Robbins ME. Radiation-induced cognitive impairment-from bench to bedside. Neuro-Oncology. 2012;14(SUPPL.4):iv37-iv44. doi:I0.1093/neuonc/nos196

3. Lee YW, Cho HJ, Lee WH, Sonntag WE. Whole brain radiation-induced cognitive impairment: pathophysiological mechanisms and therapeutic targets. Biomolecules \& therapeutics. 20I2;20(4):357370. doi:I0.4062/biomolther.2012.20.4.357

4. Greene-Schloesser D, Robbins ME. Radiation-induced cognitive impairment-from bench to bedside. Neuro-Oncology. 2012;14(SUPPL.4):iv37-iv44. doi:I0.1093/neuonc/nos196

5. Pääkkö E, Harila-Saari A, Vanionpää L, Himanen S, Pyhtinen J, Lanning M. White matter changes on $\mathrm{MRI}$ during treatment in children with acute lymphoblastic leukemia: correlation with neuropsychological findings. Medical and pediatric oncology. 2000;35(5):456-46I. doi:10.1002/I0969IIX(2000II0I)35:5<456::AID-MPO3>3.0.CO;2-I

6. Fujii $O$, Tsujino K, Soejima T, Yoden E, Ichimiya $Y$, Sugimura $K$. White matter changes on magnetic resonance imaging following whole-brain radiotherapy for brain metastases. Radiation Medicine. 2006;24(5):345-350. doi:10.1007/s | 1604-006-0039-9

7. Gondi V, Hermann BP, Mehta MP, Tome WA. Hippocampal dosimetry predicts neurocognitive function impairment after fractionated stereotactic radiotherapy for benign or low-grade adult brain tumors. International journal of radiation oncology, biology, physics. 2013;83(4):e487-93. doi:10.1016/j. ijrobp.2011.10.021

8. Gondi V, Tomé WA, Mehta MP. Why avoid the hippocampus? A comprehensive review. Radiotherapy and Oncology. 2010;97(3):370-376. doi:10.1016/j.radonc.2010.09.013

9. David S, Mesri HY, Bodiut VA, et al. Dose-dependent degeneration of non-cancerous brain tissue in post-radiotherapy patients: A diffusion tensor imaging study. medRxiv. Published online January I, 2019:19005157. doi:10.1101/19005157

10. Dahnke R, Yotter RA, Gaser C. Cortical thickness and central surface estimation. Neurolmage. 2013;65:336-348. doi:10.1016/j.neuroimage.2012.09.050

II. Fischl B, Dale AM. Measuring the thickness of the human cerebral cortex from magnetic resonance images. Proceedings of the National Academy of Sciences of the United States of America. 2000;97(20):II050-II055. doi:I0.1073/pnas.200033797

12. Ashburner J, Friston KJ. Voxel-Based Morphometry-The Methods. Neurolmage. 2000; I (6):805-82I. doi:10.1006/nimg.2000.0582

13. Ashburner J, Friston KJ. Unified segmentation. Neurolmage. 2005;26(3):839-851. doi:10.1016/j. neuroimage.2005.02.018

14. Nagtegaal SHJ, David S, van der Boog ATJ, Leemans A, Verhoeff JJC. Changes in cortical thickness and volume after cranial radiation treatment: A systematic review. Radiotherapy and Oncology. 2019;135:3342. doi:10.1016/j.radonc.2019.02.013

15. Karunamuni R, Bartsch H, White NS, et al. Dose-Dependent Cortical Thinning After Partial Brain Irradiation in High-Grade Glioma. International journal of radiation oncology, biology, physics. 2016:94(2):297-304. doi:10.1016/j.jirobp.2015.10.026

16. Seibert TM, Karunamuni R, Kaifi S, et al. Cerebral Cortex Regions Selectively Vulnerable to Radiation Dose-Dependent Atrophy. International Journal of Radiation Oncology Biology Physics. 2017;97(5):910918. doi:10.1016/j.jijrobp.2017.01.005

17. Seo SW, Im K, Lee J-M, et al. Cortical thickness in single- versus multiple-domain amnestic mild cognitive impairment. Neurolmage. 2007;36(2):289-297. doi:10.1016/j.neuroimage.2007.02.042

18. Garcia-Diaz Al, Segura B, Baggio HC, et al. Cortical thinning correlates of changes in visuospatial and visuoperceptual performance in Parkinson's disease: A 4-year follow-up. Parkinsonism \& Related Disorders. 2018;46:62-68. doi:I0.1016/j.parkreldis.2017.11.003

19. Penny W, Friston K, Ashburner J, Kiebel S, Nichols T. Statistical Parametric Mapping: The Analysis of Functional Brain Images.; 2007. doi:I0.1016/B978-0-12-372560-8.X5000-I 
20. Gaser C, Dahnke R. CAT-a computational anatomy toolbox for the analysis of structural MRI data. Hbm. 2016;2016(7):336-348.

21. Winkler AM, Ridgway GR, Webster MA, Smith SM, Nichols TE. Permutation inference for the general linear model. Neurolmage. 2014;92:38I-397. doi:10.1016/j.neuroimage.2014.01.060

22. Nichols T, Holmes A. Nonparametric Permutation Tests for Functional Neuroimaging. Human Brain Function: Second Edition. 2003;25(August 1999):887-9I0. doi:I0.1016/B978-0I226484I-0/50048-2

23. Holmes AP, Blair RC, Watson JD, Ford I. Nonparametric analysis of statistic images from functional mapping experiments. Journal of cerebral blood flow and metabolism : official journal of the International Society of Cerebral Blood Flow and Metabolism. 1996;16(I):7-22. doi:10.1097/00004647-19960100000002

24. Wasserstein RL, Lazar NA. The ASA Statement on p -Values: Context, Process, and Purpose. The American Statistician. 2016;70(2):129-133. doi:10.1080/00031305.2016.1154108

25. Smith SM, Nichols TE. Threshold-free cluster enhancement: Addressing problems of smoothing, threshold dependence and localisation in cluster inference. Neurolmage. 2009;44(I):83-98. doi:10.1016/j.neuroimage.2008.03.061

26. Eklund A, Nichols TE, Knutsson $\mathrm{H}$. Cluster failure: Why fMRI inferences for spatial extent have inflated false-positive rates. Proceedings of the National Academy of Sciences. 2016; I 3(28):7900-7905. doi:I0.1073/pnas. 1602413/13

27. Thambisetty M, Wan J, Carass A, An Y, Prince JL, Resnick SM. Longitudinal changes in cortical thickness associated with normal aging. Neurolmage. 2010;52(4):1215-1223. doi:10.1016/j. neuroimage.2010.04.258

28. Hurtz S, Woo E, Kebets V, et al. Age Effects on Cortical Thickness in Cognitively Normal Elderly Individuals. Dementia and Geriatric Cognitive Disorders Extra. 2014;4(2):22I-227. doi:I 0.II59/000362872

29. Desikan RS, Ségonne F, Fischl B, et al. An automated labeling system for subdividing the human cerebral cortex on MRI scans into gyral based regions of interest. Neurolmage. 2006;3I (3):968-980. doi:10.1016/j.neuroimage.2006.01.021

30. Vanderah T, Gould D. Nolte's the Human Brain: An Introduction to Its Functional Anatomy. 7th ed. Elsevier; 2015.

31. Binder JR. The Wernicke area: Modern evidence and a reinterpretation. Neurology. 2015;85(24):21702175. doi:I0.1212/WNL.0000000000002219

32. Koenigs M, Barbey AK, Postle BR, Grafman J. Superior parietal cortex is critical for the manipulation of information in working memory. The Journal of neuroscience: the official journal of the Society for Neuroscience. 2009;29(47): 14980-|14986. doi:10.1523/JNEUROSCI.3706-09.2009

33. Salat DH. Thinning of the Cerebral Cortex in Aging. Cerebral Cortex. 2004;14(7):721-730. doi:10.1093/ cercor/bhh032

34. Du A-T, Schuff N, Kramer JH, et al. Different regional patterns of cortical thinning in Alzheimer's disease and frontotemporal dementia. Brain. 2007;130(4):1159-1166. doi:10.1093/brain/awm016

35. Yin C, Li S, Zhao W, Feng J. Brain imaging of mild cognitive impairment and Alzheimer's disease. Neural regeneration research. 2013;8(5):435-444. doi:10.3969/j.issn. 1673-5374.2013.05.007

36. Pereira JB, Ibarretxe-Bilbao N, Marti M-J, et al. Assessment of cortical degeneration in patients with Parkinson's disease by voxel-based morphometry, cortical folding, and cortical thickness. Human Brain Mapping. 2012;33(II):252I-2534. doi:10.1002/hbm.21378

37. Lim HK, Jung WS, Ahn KJ, et al. Regional cortical thickness and subcortical volume changes are associated with cognitive impairments in the drug-naive patients with late-onset depression. Neuropsychopharmacology. 2012;37(3):838-849. doi:I0.1038/npp.2011.264

38. Peterson BS, Warner V, Bansal R, et al. Cortical thinning in persons at increased familial risk for major depression. Proceedings of the National Academy of Sciences. 2009;106(15):6273-6278. doi:10.1073/ pnas.0805311106

39. Lumniczky K, Szatmári T, Sáfrány G. Ionizing Radiation-Induced Immune and Inflammatory Reactions in the Brain. Frontiers in immunology. 2017;8:517. doi:I0.3389/fimmu.2017.00517

40. Fike JR, Cann CE, Turowski K, et al. Radiation dose response of normal brain. International Journal of Radiation Oncology*Biology*Physics. 1988;14(I):63-70. doi:10.1016/0360-3016(88)90052-I 
41. Wong CS, Van der Kogel AJ, Shun Wong C, der Kogel AJ. Mechanisms of radiation injury to the central nervous system: implications for neuroprotection. Molecular interventions. 2004;4(5):273-284. doi:| 0.II24/mi.4.5.7

42. Nagtegaal S, David S, Philippens M, Leemans A, Verhoeff J. Any Radiation Dose to the Hippocampus Leads to Local Volume Loss in a Dose-Time-Dependent Way. International Journal of Radiation Oncology*Biology*Physics. 2019; I05(I):EI04-EI05. doi:I0.1016/j.jirobp.2019.06.2302

43. Huynh-Le M-P, Karunamuni R, Moiseenko V, et al. Dose-dependent atrophy of the amygdala after radiotherapy. Radiotherapy and Oncology. 2019;136:44-49. doi:10.1016/j.radonc.2019.03.024

44. Siegel RL, Miller KD, Jemal A. Cancer statistics, 2019. CA: A Cancer Journal for Clinicians. 2019;69(I):734. doi:I0.3322/caac.2155|

45. Karunamuni RA, Moore KL, Seibert TM, et al. Radiation sparing of cerebral cortex in brain tumor patients using quantitative neuroimaging. Radiotherapy and oncology : journal of the European Society for Therapeutic Radiology and Oncology. 2016;1 I8(I):29-34. doi:10.1016/j.radonc.2016.01.003

46. Murzin VL, Woods K, Moiseenko V, et al. 4pi plan optimization for cortical-sparing brain radiotherapy. Radiotherapy and oncology: journal of the European Society for Therapeutic Radiology and Oncology. Published online March 2018. doi:10.1016/j.radonc.2018.02.011

47. McDonald BC, Conroy SK, Smith DJ, West JD, Saykin AJ. Frontal gray matter reduction after breast cancer chemotherapy and association with executive symptoms: A replication and extension study. Brain Behav Immun. Published online 2012.

48. Stouten-Kemperman MM, de Ruiter MB, Koppelmans $\vee$, Boogerd W, Reneman L, Schagen SB. Neurotoxicity in breast cancer survivors $\geq 10$ years post-treatment is dependent on treatment type. Brain imaging and behavior. 2015;9(2):275-284. doi:I0. I007/s I 682-014-9305-0

49. Genschaft M, Huebner T, Plessow F, et al. Impact of Chemotherapy for Childhood Leukemia on Brain Morphology and Function. Beaulieu C, ed. PLoS ONE. 2013;8(1I):e78599. doi:10.1371/journal. pone.0078599

50. Blommaert J, Schroyen G, Vandenbulcke M, et al. Age-dependent brain volume and neuropsychological changes after chemotherapy in breast cancer patients. Human Brain Mapping. Published online August 21, 2019:hbm.24753. doi:10.1002/hbm.24753 


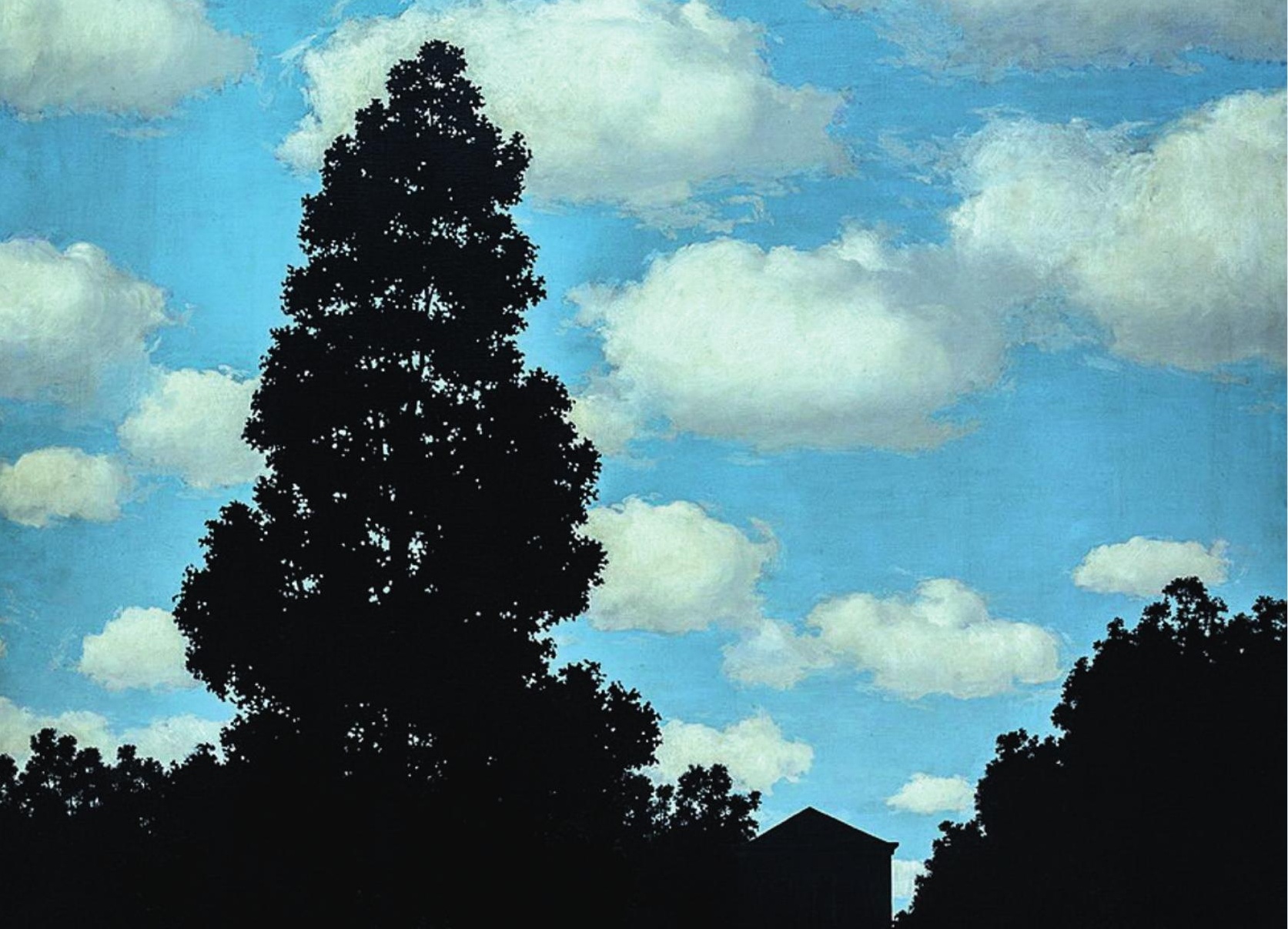

4t

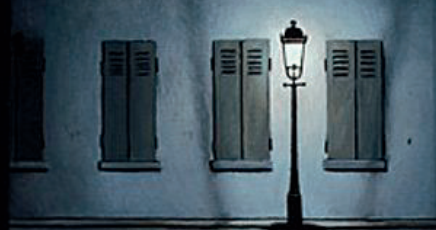




\section{Chapter 4}

Dose-dependent volume loss in subcortical deep grey matter structures after cranial radiotherapy

S.H.J. Nagtegaal, S. David, M.E.P. Philippens, T.J. Snijders, A. Leemans, J.J.C. Verhoeff

Clinical and translational radiation oncology 26 (2021): 35-4I 


\section{ABSTRACT}

\section{Background and purpose}

The relation between radiotherapy (RT) dose to the brain and morphological changes in healthy tissue has seen recent increased interest. There already is evidence for changes in the cerebral cortex and white matter, as well as selected subcortical grey matter (GM) structures. We studied this relation in all deep GM structures, to help understand the aetiology of post-RT neurocognitive symptoms.

\section{Materials and methods}

We selected 3 I patients treated with RT for grade II-IV glioma. Pre-RT and I year postRT 3D TI-weighted MRIs were automatically segmented, and the changes in volume of the following structures were assessed: amygdala, nucleus accumbens, caudate nucleus, hippocampus, globus pallidus, putamen, and thalamus. The volumetric changes were related to the mean RT dose received by each structure. Hippocampal volumes were entered into a population-based nomogram to estimate hippocampal age.

\section{Results}

A significant relation between RT dose and volume loss was seen in all examined structures, except the caudate nucleus. The volume loss rates ranged from 0.16-1.37\%/Gy, corresponding to 4.9-41.2\% per 30 Gy. Hippocampal age, as derived from the nomogram, was seen to increase by a median of II years.

\section{Conclusion}

Almost all subcortical GM structures are susceptible to radiation-induced volume loss, with higher volume loss being observed with increasing dose. Volume loss of these structures is associated with neurological deterioration, including cognitive decline, in neurodegenerative diseases. To support a causal relationship between radiation-induced deep GM loss and neurocognitive functioning in glioma patients, future studies are needed that directly correlate volumetrics to clinical outcomes. 


\section{INTRODUCTION}

Irradiation of healthy brain tissue can lead to anatomical and functional deficits, a phenomenon known as radiation-induced brain injury. This can lead to a variety of symptoms, with especially cognitive and executional impairments leading to a marked decrease in the patient's quality of life after radiation therapy (RT)., ${ }^{1,2}$

With the advent of high-resolution brain imaging, the interest in morphological changes after RT has increased. The cerebral cortex has been shown to be susceptible to radiationinduced thinning, especially in areas associated with cognitive functioning. ${ }^{3-6}$ Thinning rates are found to be dose-dependent, meaning that a higher dose leads to a further diminished cortex. Similarly, diffusion tensor imaging has shown that white matter shows dose-dependent changes in several metrics after RT. ${ }^{7}$ Finally, two grey matter structures, the hippocampus ${ }^{8,9}$ and the amygdala, ${ }^{10}$ show susceptibility to radiation damage, again with higher volume changes with increasing dose. Furthermore, the dose to the hippocampus has been shown to negatively affect neurocognitive outcome after RT."

Less is known about the susceptibility to radiation damage of other subcortical grey matter structures, such as the nucleus accumbens, caudate nucleus, globus pallidus, putamen, and thalamus. Atrophy of these deep GM structures is associated with impaired cognitive function in patients with degenerative brain diseases as well as healthy ageing. ${ }^{12-14}$ This relation is most pronounced in Alzheimer's disease, with the volume of all mentioned structures, with the exception of globus pallidus, being associated with cognitive impairment. ${ }^{15-17}$ Globus pallidus volume in its turn is associated with cognitive outcomes in Huntington's disease and age-related cognitive impairments. ${ }^{18,19}$

Volume changes in these structures are associated with cognitive outcomes, and the cause of post-RT cognitive decline needs to be elucidated. Therefore, we examined the relation between post-RT subcortical GM volume changes and RT dose.

\section{METHODS}

\section{Patient selection and data collection}

Patients who were treated with photon intensity-modulated radiation therapy RT for newly discovered grade II-IV glioma at the department of Radiation Oncology in 2016 and 2017 were retrospectively identified. Criteria for inclusion were: treatment planning CT and MRI present, with isotropic high resolution; survival > 270 days after start of RT; and availability of at least I follow-up MRI between 270 days and 360 days after start of RT, and with isotropic high resolution. Patients were excluded in case of tumour progression 
or recurrence between baseline and follow-up. Clinical MRI and CT scans made for RT treatment planning, all follow-up MRIs, and clinical and demographic characteristics were extracted from patient records. The need for informed consent for this retrospective study was waived by our institutional review board (\#|8/274).

\section{Image acquisition}

For every patient the pre-RT CT and MRI were collected, as well as all available follow-up MRIs. RT planning CT scans were acquired on a Brilliance Big bore scanner (Philips Medical Systems, Best, The Netherlands), with a tube potential of $120 \mathrm{kVp}$, with a matrix size of $512 \times 512$ and $0.65 \times 0.65 \times 3.0 \mathrm{~mm}$ voxel size. MR images were acquired on a $3 T$ Philips Ingenia scanner (Philips Healthcare, Best, The Netherlands) as part of routine clinical care. TI-weighted MR images were acquired with a 3D turbo field echo (TFE) sequence without gadolinium enhancement with the following parameters: $\mathrm{TR}=8.1 \mathrm{~ms}$, TE $=3.7 \mathrm{~ms}$, flip angle $=8^{\circ}$, matrix: $207 \times 289 \times 213$, and a reconstructed voxel resolution of $1 \times 0.96 \times 0.96$ $\mathrm{mm}$.

\section{Image processing}

A graphical overview of the image processing pipeline is shown in Figure I. All imaging data was processed with Statistical Parametric Mapping (SPMI2, v7487) ${ }^{20}$ Computational Anatomy Toolbox (CATI2.6 rl450) ${ }^{21}$ and in-house algorithms developed in MATLAB (Mathworks, Natick, Massachusetts, USA). Image processing was done in concordance to our own previously published criteria, ${ }^{3}$ amended for the current research question. More detailed image processing methods can be found in our previous work. ${ }^{4}$

In brief, the cropped CT image with the associated dose and planning target volume (PTV) maps were registered to the TI MR images, resulting in the CT image and the MRIs being in the same space. Next, the rigidly coregistered TIs were processed with CATI2's segmentation pipeline.

Deep GM structure volumes were estimated with CATI2 using the fully automated volume estimation method using the labels from the Neuromorphometrics atlas (Neuromorphometrics Inc., Somerville, Massachusetts, USA). The following structures were examined: amygdala, nucleus accumbens, caudate nucleus, hippocampus, globus pallidus, putamen, and thalamus. Figure $\mathbf{2}$ shows the anatomical location of the structures on axial TI MRI, as well as in a 3D rendering. This resulted in 14 volumes per patient, as the GM volumes for the left and right hemisphere were separately estimated. 


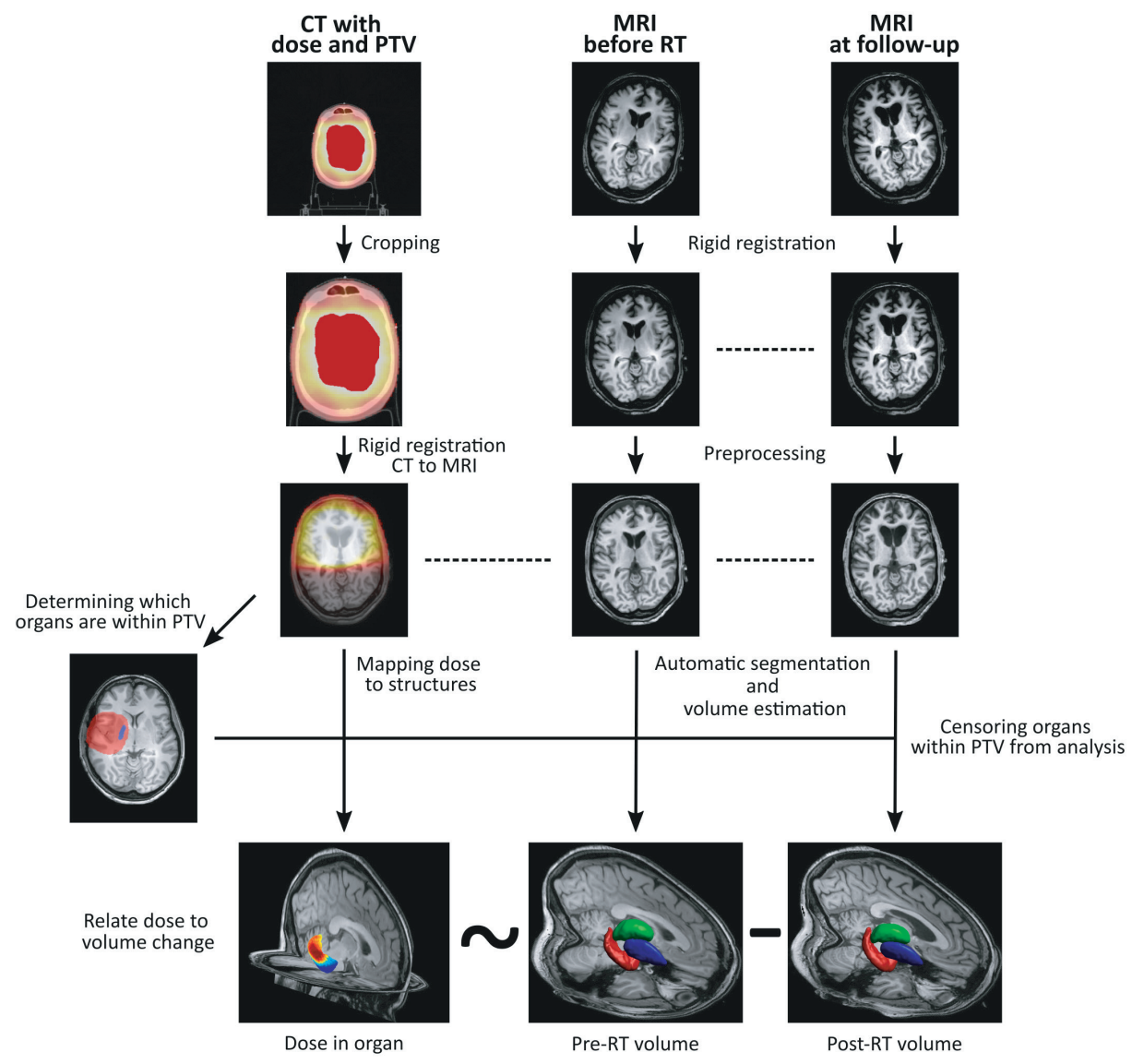

Figure I Pipeline of image processing. Left column: dose (colour gradient) and PTV (red shading) are extracted from CT. Middle and right column: organ volume is estimated from processed MRIs before RT and at follow-up. CT and MRI are registered to each other (dotted lines), and organs within PTV are censored from analysis. Finally applied dose is related to the change in organ volume. Note that the images used are used illustratively, and do not represent a single case.

For the primary analysis, we analysed the difference in volume between baseline and I year follow-up. The latter was defined as the time point closest to 360 days after start of RT for which an MRI was available.

The within-subject difference in deep GM volume was calculated by subtracting the baseline and the I-year follow-up volume. In every subject the deep GM organs included in the PTV were censored from analysis, to avoid spurious volume-dose relations originating from segmentation errors due to damage around the tumour. ${ }^{22}$ If the residual damage (e.g. oedema, surgical scarring, tumour bed) extended beyond the PTV in either the baseline or the follow-up images, then the affected subject was removed from the analysis. 


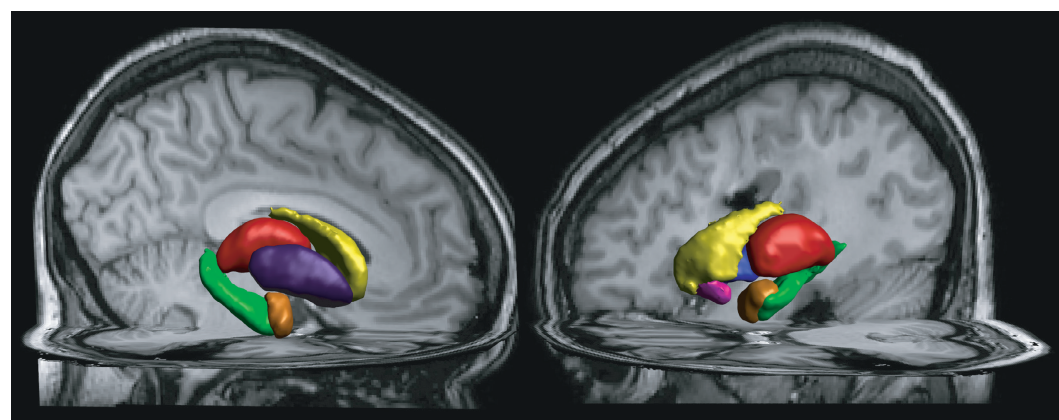

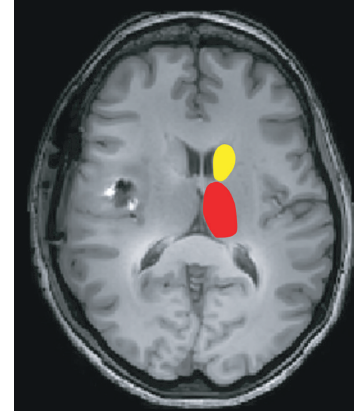

Amygdala

- Caudate nucleus

Hippocampus

\section{Nucleus accumbens}

Figure 2 A 3D rendering and axial MR images showing the subcortical grey matter structures being analysed.

\section{Statistical analysis}

We correlated the change in deep GM volume after I year with the mean dose received by each subcortical structure. Statistical comparison of deep GM volume change and dose correlation was carried out with a permutation test with 10,000 iterations performed with the permutation analysis of linear models (PALM) toolbox in Matlab. ${ }^{23-25}$ Significance of a correlation was set at $\mathrm{P}_{\text {corr }}<0.05$, with use of family-wise error rate (FWER) adjustment to correct for multiple comparisons. All further

presented p-values are FWER-corrected. Age at the time of the diagnosis and sex of the patients were included as nuisance regressors. 
To assess whether administration of chemotherapy has an effect on the relation between dose and volume, a sensitivity analysis was performed in which chemotherapy was added as a covariate to the permutation test, again with FWER-adjustment.

\section{Hippocampal nomograms}

To put volumetric changes of the hippocampus into context, the pre-RT and post-RT volumes were entered into a nomogram of hippocampal volume across age groups. ${ }^{26,27}$ This nomogram is based on MRI data from 19,700 healthy participants from the UK Biobank. We did this in two ways: I) the patients' new "hippocampal age" was determined based on its volume after RT and the percentile in the nomogram at baseline; this was then compared to the actual age at baseline, and 2) we assessed whether there was a change in a patient's hippocampal volume percentile within the population between the pre-RT and post-RT scans. For this analysis we used not only the I-year post-RT MRI, but all available followup MRIs. Due to the age range of the nomograms, only patients aged 52 to 72 could be entered into the nomogram. When hippocampal volumes at follow-up were below the limits of the nomogram, the hippocampal age was set at the maximum age that could be derived from the reference dataset (i.e. 72).

\section{RESULTS}

\section{Participants}

Of all the patients treated with RT for glioma in 2016 and 2017, thirty-one patients were eligible for inclusion in the current analysis. A flow-chart of study inclusion is shown in Supplementary Figure I. Extensive damage outside the censored PTV area on baseline MRI meant exclusion of one case. Baseline characteristics are shown in Table I. Median follow-up time of the used MRI assessments was 319 days, with a range of $270-360$.

\section{Subcortical GM volume}

Significant dose-dependent volume loss I year after RT was observed in all examined structures, except for caudate nucleus. Rates of volume loss vary from 0.16 to $1.37 \%$ per Gy (corresponding to $4.9 \%$ and $41.2 \%$ per $30 \mathrm{~Gy}$ ), and are shown for all structures in Table 2. Scatterplots of the organs in which a significant relation between RT dose and volume loss was seen are shown in Figure 3. Doses received per structure are shown in Supplementary Table 2. 
Table I Baseline characteristics of included patients

\begin{tabular}{ll}
\hline & $\mathbf{N}$ (total $\mathbf{n}=\mathbf{3 I}$ ) \\
\hline Age (mean; SD) & $50( \pm 15)$ \\
Sex & \\
Male & $19(61.3 \%)$ \\
Female & $12(38.7 \%)$ \\
WHO grade & \\
II & $12(38.7 \%)$ \\
III & $6(19.4 \%)$ \\
IV & $13(41.9 \%)$ \\
Tumour type & \\
Astrocytoma, IDH-mutant & $13(41.9 \%)$ \\
Astrocytoma, IDH-wildtype & $3(9.6 \%)$ \\
Glioblastoma, IDH-wildtype & $9(29.0 \%)$ \\
Other & $6(19.6 \%)$ \\
Prescribed dose & \\
$28 \times 1.8 G y=50.4$ Gy & $11(35.5 \%)$ \\
$30 \times 1.8 G y=54$ Gy & $2(6.5 \%)$ \\
$30 \times 2.0 G y=60$ Gy & $18(58.1 \%)$ \\
Concurrent or sequential systemic therapy & \\
None & $5(16.1 \%)$ \\
Temozolomide & $21(67.7 \%)$ \\
PCV & $4(12.9 \%)$ \\
\hline
\end{tabular}

$\mathrm{PCV}=$ procarbazine, lomustine and vincristine

The sensitivity analysis done to assess the effect of chemotherapy on this relation is shown in Supplementary Table 2. It did not result in a change in direction or effect size of the results, and chemotherapy administration did not significantly affect GM volume. 


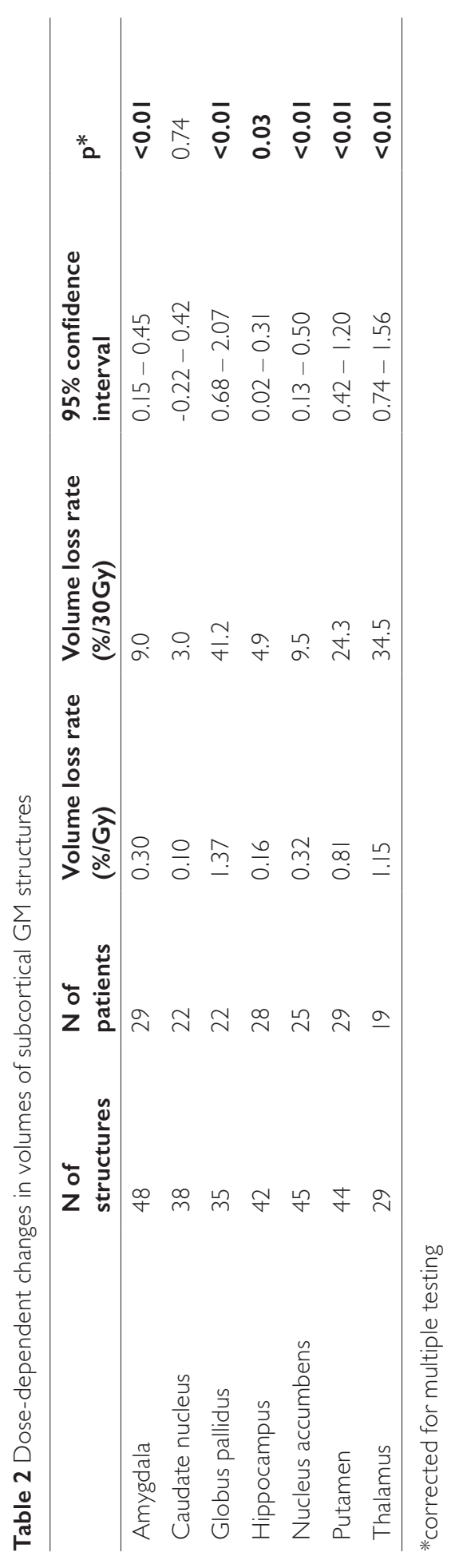




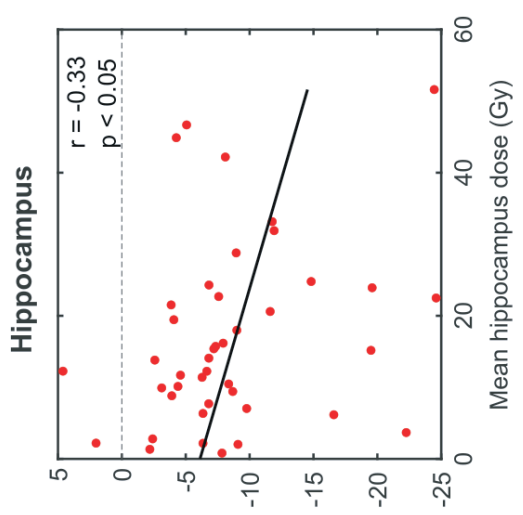

(\%) \لу גәне деә К L әбиечо әшпю^

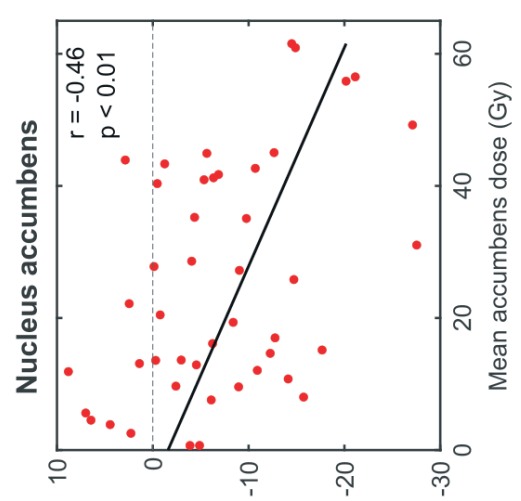

(\%) دу дәне деә $K_{L}$ әбиечо әщпю^

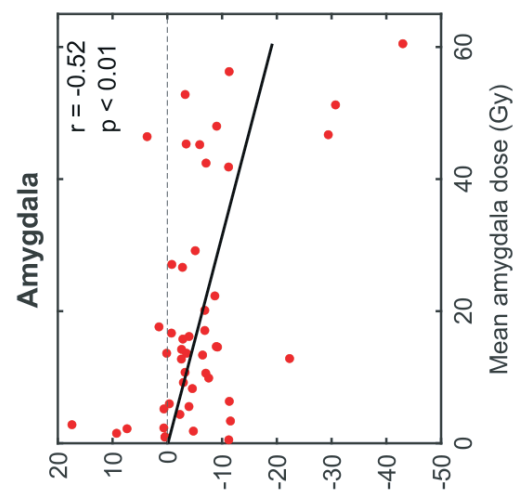

(\%) цу дәне деәК L әбиечо әшпю^

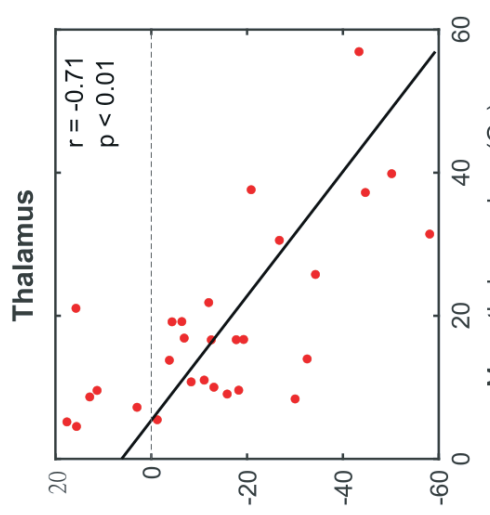

(\%) цل دәџе леәК । әбиечо әшпюภ

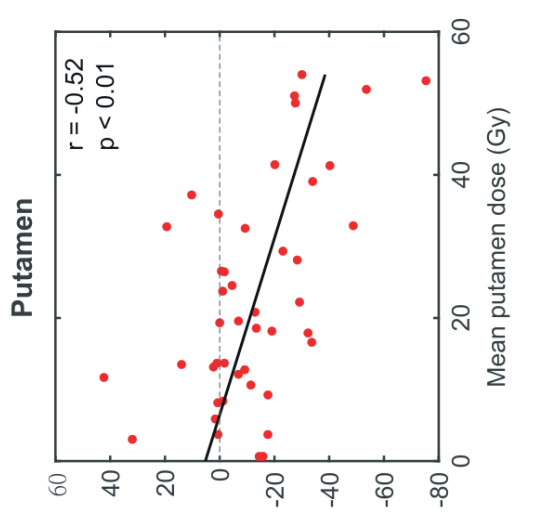

(\%) цل ләще леәК । әбиечо әшп।о

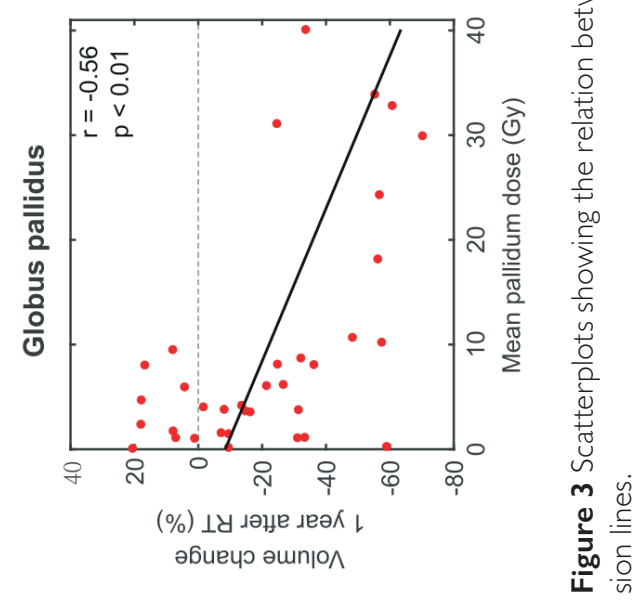




\section{Hippocampal volume nomograms}

In this cohort 22 patients were within the age range of 52 to 72 , and thus were entered into the nomograms from the UK Biobank, which are shown in Figure 4. All patients show an overall increase in hippocampal age, with a median increase of eleven years (range 2 - 20 years). Accordingly, the percentile within the nomogram dropped for all patients, meaning that their hippocampal volume shows a decrease compared to their peers of the same age.
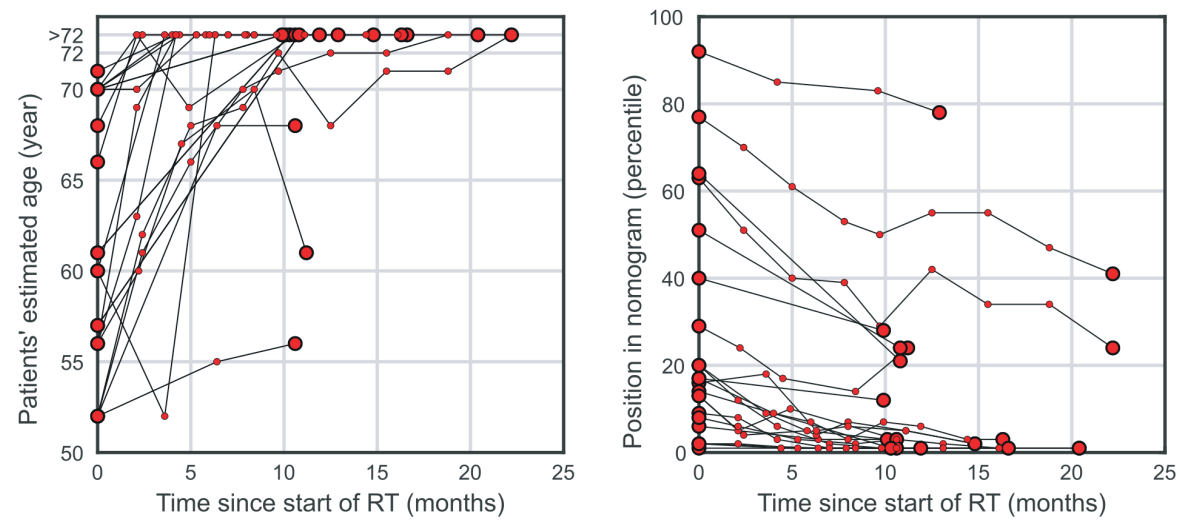

Figure 4 Change in patients' hippocampal age (left) and position within the nomogram (right) based on the UK Biobank [24], estimated using all available clinical MRIs. Hippocampal age saturates at the top of the graph because age within the nomogram has a maximum of 72 . Large points denote first and last available MRIs, small points those in between.

\section{DISCUSSION}

When analysing structures outside of the treated PTVs, we have found that all subcortical deep grey matter structures, with the exception of caudate nucleus, show dose-dependent volume loss I year after RT. For the hippocampus, we have also shown that, based on data from the normal population, its volume-based age increases with up to twenty years during the year post-radiation.

The amygdala and hippocampus have shown to be susceptible to radiation damage in previous studies. Seibert et al. ${ }^{8}$ studied MRIs before and one year after RT of 52 patients with primary brain tumours. Automatic segmentation of the hippocampus was followed by relating the difference in volume to the mean dose received. They found a significant correlation with a Pearson correlation coefficient of -0.24 . Furthermore, they found that the hippocampus showed significant volume loss after high-dose RT (defined as >40 Gy). This in contrast to low-dose (<10 Gy), which showed no significant relation with post-RT 
volume. A linear mixed-effects model resulted in a volume loss rate of $0.13 \% / G y$, which is similar to our observed loss rate of $0.16 \% / \mathrm{Gy}$.

The volumetric changes in the amygdala after RT have been studied by Huynh-Le et al. ${ }^{10}$ in the same cohort of 52 patients. A significant Pearson correlation of -0.28 was found for amygdala volume and mean dose, with a volume loss rate of $0.17 \% / G y$. The difference to our findings of a correlation coefficient of -0.52 and volume loss rate of $0.30 \% / G y$ could be explained by the difference in censoring method. They censored amygdalae manually when a visual inspection deemed the segmentation to be poor, whereas we censored more strictly by censoring any organ within the PTV. This meant that they have more data points within higher dose regions, which could have led to a different slope and correlation coefficient.

Finally, a link between post-RT hippocampal volume and neurocognitive outcomes was found in primary brain tumours by Tringale et al. ${ }^{9}$ They found that, in addition to diffusion biomarkers, a smaller right hippocampal volume was associated with poorer visuospatial memory performance in the 12 months after RT.

A link between the volumes of these structures and cognitive outcomes has been thoroughly examined in other brain diseases. Particularly In Alzheimer's disease, available evidence points towards a strong relation between subcortical GM structures and cognitive impairments for each of the structures we studied except for globus pallidus. ${ }^{15-17}$ Furthermore, cognitive impairments in Parkinson's disease, ${ }^{28,29}$ multiple sclerosis, ${ }^{30}$ Huntington disease, ${ }^{18}$ as well as in normal ageing, ${ }^{13}$ have been linked with the volume of at least one of the subcortical GM structures. Supplementary Table 3 gives an overview of some available literature per GM structure.

The effect of hippocampal dose and neurocognitive outcomes was first shown by Gondi et al." They showed that radiation dose of $>7.3$ Gy to $40 \%$ of the bilateral hippocampi was associated with an impairment in Wechsler Memory Scale-III Word List delayed recall. The same group conducted phase II and phase III trials, the latter studying the effect of whole brain radiotherapy (WBRT) with or without hippocampal avoidance. . $^{31,32}$ They found hippocampal avoidance WBRT, in combination with the $\mathrm{N}$-methyl-D-aspartate inhibitor memantine, preserves cognitive function while maintaining the same overall and progression-free survival.

In this study the investigation of caudate nucleus volume change in relation to the local dose was inconclusive. One explanation could be that the quality of the segmentation is region dependent. While generally the segmentations of SPM/CATI2 is highly reproducible, ${ }^{33-35}$ among the investigated regions caudate requires the largest sample size to achieve the same 
statistical power as compared to other regions. ${ }^{36}$ The caudate nucleus shares a relatively large interface with the ventricles (Figure 2), making it highly susceptible to partial voluming artefacts, which may lead to errors in segmentation.

Our results challenge us to reconsider the currently used sparing strategies in radiation treatment of brain tumours. Presently, hippocampal sparing RT has been adopted in several institutions. However, sparing the dose in the hippocampus leads to higher doses in surrounding cerebral tissues, which we have shown to be susceptible to radiationinduced damage as well. ${ }^{37}$ Future research has to focus on the relation between clinical outcomes (including cognitive and motor function) and morphologic changes, both in the entire brain and in selected structures. This way we can conclusively say which structures should be avoided in RT planning to prevent radiation-induced damage. Specific sparing of healthy brain is possible with novel techniques such as proton therapy and VMAT. Especially in intensity-modulated proton therapy, doses to organs at risk can be optimally reduced, ${ }^{38}$ meaning this technique may prove useful in preventing post-RT cognitive decline. Additionally, the relative biological effectiveness (RBE) for several substructures is still unknown and may impact the effect of radiation. This could lead to improved cognition and quality of life in patients undergoing treatment for brain tumours.

There are several limitations to this study. Firstly, we have a relatively limited sample size due to the strenuous inclusion criteria. However, these criteria ensure that the quality of the imaging used in analysis are optimal, meaning more reliable and replicable results. The censoring of the PTV also means exclusion of several subcortical GM structures, but this again is to ensure reliable automated measurements. Attempts could have been made to manually delineate these structures, but this would have added an extra variable to the dose/volume relation (manual vs automatic segmentation in high and lower dose, respectively). Additionally, it is unclear which method gives the most reliable results in patients who underwent RT. In previous works, ${ }^{39-41}$ the automated segmentation method used in the current study was rigorously compared to manual segmentation of subjects' TI MRI data as well as brain phantoms representing a wide range of settings (noise, artefacts, etc.). It was found that CATI2 performed on a comparable level versus manual segmentation in healthy subjects as well as patients with ischemic stroke or temporal lobe epilepsy, suggesting it is reliable for segmentation in RT patients.

Another consideration is that susceptibility to radiation-induced volume loss of brain tissue might differ between patients. As each patient provides multiple organs for examination, this could have impacted the found results. We could not correct for this in the current study, as our sample size limited our ability to apply multilevel modelling of the dose/volume 
relation. For similar reasons, we were unable to properly model the longitudinal changes over time, but looked only at the change I year after RT.

Secondly, the patients in our cohort did not only undergo RT. Many also received chemotherapy, which has been linked to cerebral changes in non-neurological malignancies. ${ }^{42,43}$ Our analysis focussed on the association between RT dose and volume, and by relating these two factors to each other we have limited the effect of chemotherapy as much as possible. Furthermore, a sensitivity analysis including chemotherapy in the model did not give different results, suggesting its role is limited.

Finally, the absence of neurocognitive outcome data from this cohort means we cannot yet give clinical recommendations on which organs to spare.

In conclusion, subcortical grey matter structures show susceptibility to dose-dependent volume loss after radiotherapy. If neurocognitive outcomes are related to this phenomenon, current RT strategies need to be revised, in order to improve patients' quality of life after cancer treatment. 


\section{REFERENCES}

I. Makale MT, McDonald CR, Hattangadi-Gluth JA, Kesari S. Mechanisms of radiotherapy-associated cognitive disability in patients with brain tumours. Nature Reviews Neurology. 2017;13(I):52-64. doi:10.1038/nrneurol.2016.185

2. Greene-Schloesser D, Robbins ME. Radiation-induced cognitive impairment-from bench to bedside. Neuro-Oncology. 2012;14(SUPPL.4):iv37-iv44. doi:I0.1093/neuonc/nos196

3. Nagtegaal SHJ, David S, van der Boog ATJ, Leemans A, Verhoeff JJC. Changes in cortical thickness and volume after cranial radiation treatment: A systematic review. Radiotherapy and Oncology. 2019;135:3342. doi:10.1016/j.radonc.2019.02.013

4. Nagtegaal SHJ, David S, Snijders TJ, Philippens MEP, Leemans A, Verhoeff JJC. Effect of radiation therapy on cerebral cortical thickness in glioma patients: treatment-induced thinning of the healthy cortex. Neuro-Oncology Advances. 2020;2(I). doi:I0.1093/noajnl/vdaa060

5. Seibert TM, Karunamuni R, Kaifi S, et al. Cerebral Cortex Regions Selectively Vulnerable to Radiation Dose-Dependent Atrophy. International Journal of Radiation Oncology Biology Physics. 2017;97(5):910918. doi:10.1016/j.ijrobp.2017.01.005

6. Karunamuni R, Bartsch H, White NS, et al. Dose-Dependent Cortical Thinning After Partial Brain Irradiation in High-Grade Glioma. International journal of radiation oncology, biology, physics. 2016;94(2):297-304. doi:10.1016/j.ijrobp.2015.10.026

7. David S, Mesri HY, Bodiut VA, et al. Dose-dependent degeneration of non-cancerous brain tissue in post-radiotherapy patients: A diffusion tensor imaging study. medRxiv. Published online January I, 2019:19005157. doi:10.1101/19005157

8. Seibert TM, Karunamuni R, Bartsch H, et al. Radiation Dose-Dependent Hippocampal Atrophy Detected With Longitudinal Volumetric Magnetic Resonance Imaging. International Journal of Radiation Oncology*Biology*Physics. 2017;97(2):263-269. doi:I0.1016/j.ijrobp.2016.10.035

9. Tringale KR, Nguyen TT, Karunamuni R, et al. Quantitative Imaging Biomarkers of Damage to Critical Memory Regions Are Associated With Post-Radiation Therapy Memory Performance in Brain Tumor Patients. In: International Journal of Radiation Oncology Biology Physics. Vol 105. Elsevier Inc.; 2019:773783. doi:10.1016/j.ijrobp.2019.08.003

10. Huynh-Le M-P, Karunamuni R, Moiseenko V, et al. Dose-dependent atrophy of the amygdala after radiotherapy. Radiotherapy and Oncology. 2019;136:44-49. doi:10.1016/j.radonc.2019.03.024

II. Gondi V, Hermann BP, Mehta MP, Tome WA. Hippocampal dosimetry predicts neurocognitive function impairment after fractionated stereotactic radiotherapy for benign or low-grade adult brain tumors. International journal of radiation oncology, biology, physics. 2013;83(4):e487-93. doi:10.1016/j. ijrobp.2011.10.021

12. Pagnozzi AM, Fripp J, Rose SE. Quantifying deep grey matter atrophy using automated segmentation approaches: A systematic review of structural MRI studies. Neurolmage. 2019;201:116018. doi:10.1016/j. neuroimage.2019.116018

13. Hughes EJ, Bond J, Svrckova P, et al. Regional changes in thalamic shape and volume with increasing age. Neurolmage. 2012;63(3): |134-I|42. doi:I0.1016/j.neuroimage.2012.07.043

14. Zanchi D, Giannakopoulos P, Borgwardt S, Rodriguez C, Haller S. Hippocampal and Amygdala Gray Matter Loss in Elderly Controls with Subtle Cognitive Decline. Frontiers in Aging Neuroscience. 20 17;9. doi:10.3389/fnagi.2017.00050

15. de Jong LW, van der Hiele K, Veer IM, et al. Strongly reduced volumes of putamen and thalamus in Alzheimer's disease: an MRI study. Brain: a journal of neurology. 2008;131(Pt 12):3277-3285. doi:10.1093/brain/awn278

16. Nie X, Sun Y, Wan S, et al. Subregional Structural Alterations in Hippocampus and Nucleus Accumbens Correlate with the Clinical Impairment in Patients with Alzheimer's Disease Clinical Spectrum: Parallel Combining Volume and Vertex-Based Approach. Frontiers in neurology. 20 17;8:399. doi:10.3389/fneur.2017.00399

17. Yi H-A, Möller C, Dieleman N, et al. Relation between subcortical grey matter atrophy and conversion from mild cognitive impairment to Alzheimer's disease. Journal of Neurology, Neurosurgery \& Psychiatry. 2016:87(4):425-432. doi:10.1136/jnnp-2014-309105 
18. Aylward EH, Harrington DL, Mills JA, et al. Regional atrophy associated with cognitive and motor function in prodromal Huntington disease. Journal of Huntington's disease. 2013;2(4):477-489. doi:I0.3233/JHD-I30076

19. Valdés Hernández MC, Clark R, Wang S-H, et al. The striatum, the hippocampus, and short-term memory binding: Volumetric analysis of the subcortical grey matter's role in mild cognitive impairment. Neurolmage: Clinical. 2020;25:102158. doi:10.1016/j.nicl.2019.102158

20. Penny W, Friston K, Ashburner J, Kiebel S, Nichols T. Statistical Parametric Mapping: The Analysis of Functional Brain Images.; 2007. doi:1 0. I016/B978-0-12-372560-8.X5000-I

21. Gaser C, Dahnke R. CAT-a computational anatomy toolbox for the analysis of structural MRI data. Hbm. 2016;2016(7):336-348.

22. Visser M, Petr J, Müller DMJ, et al. Accurate MR Image Registration to Anatomical Reference Space for Diffuse Glioma. Frontiers in Neuroscience. 2020;14. doi:I0.3389/fnins.2020.00585

23. Winkler AM, Ridgway GR, Webster MA, Smith SM, Nichols TE. Permutation inference for the general linear model. Neurolmage. 2014;92:38I-397. doi:10.1016/j.neuroimage.2014.01.060

24. Nichols T, Holmes A. Nonparametric Permutation Tests for Functional Neuroimaging. Human Brain Function: Second Edition. 2003;25(August 1999):887-9I0. doi: I0.1016/B978-012264841-0/50048-2

25. Holmes AP, Blair RC, Watson JD, Ford I. Nonparametric analysis of statistic images from functional mapping experiments. Journal of cerebral blood flow and metabolism : official journal of the International Society of Cerebral Blood Flow and Metabolism. 1996;16(I):7-22. doi:10.1097/00004647-19960I00000002

26. Nobis L, Manohar SG, Smith SM, et al. Hippocampal volume across age: Nomograms derived from over 19,700 people in UK Biobank. Neurolmage Clinical. 2019;23:I01904. doi:I0.1016/j.nicl.2019.101904

27. Alfaro-Almagro F, Jenkinson M, Bangerter NK, et al. Image processing and Quality Control for the first 10,000 brain imaging datasets from UK Biobank. Neurolmage. 2018;166:400-424. doi:10.1016/j. neuroimage.2017.10.034

28. Hanganu A, Bedetti C, Degroot C, et al. Mild cognitive impairment is linked with faster rate of cortical thinning in patients with Parkinson's disease longitudinally. Brain : a journal of neurology. 2014;137(Pt 4):I I20- | 229. doi:10.1093/brain/awu036

29. Hünerli D, Emek-Savaş DD, Çavuşoğlu B, Dönmez Çolakoğlu B, Ada E, Yener GG. Mild cognitive impairment in Parkinson's disease is associated with decreased P300 amplitude and reduced putamen volume. Clinical Neurophysiology. 2019;130(8):I208-1217. doi:10.1016/j.clinph.2019.04.314

30. Rojas JI, Murphy G, Sanchez F, et al. Thalamus volume change and cognitive impairment in early relapsing-remitting multiple sclerosis patients. The neuroradiology journal. 2018;31(4):350-355. doi:10.1177/1971400918781977

31. Gondi V, Pugh SL, Tome WA, et al. Preservation of memory with conformal avoidance of the hippocampal neural stem-cell compartment during whole-brain radiotherapy for brain metastases (RTOG 0933): A phase II multi-institutional trial. Journal of Clinical Oncology. 2014;32(34):3810-38I6. doi:I0.1200/JCO.20I4.57.2909

32. Brown PD, Gondi V, Pugh S, et al. Hippocampal Avoidance During Whole-Brain Radiotherapy Plus Memantine for Patients With Brain Metastases: Phase III Trial NRG Oncology CCOOI. Journal of clinical oncology : official journal of the American Society of Clinical Oncology. Published online February 14, 2020:JCO1902767. doi:10.1200/JCO.19.02767

33. de Boer R, Vrooman HA, Ikram MA, et al. Accuracy and reproducibility study of automatic MRI brain tissue segmentation methods. Neurolmage. 2010;5I(3):1047-1056. doi:10.1016/j. neuroimage.2010.03.012

34. Shuter B, Yeh IB, Graham S, Au C, Wang SC. Reproducibility of brain tissue volumes in longitudinal studies: Effects of changes in signal-to-noise ratio and scanner software. Neurolmage. 2008;4I (2):37I379. doi: $10.1016 /$ j.neuroimage.2008.02.003

35. Guo C, Ferreira D, Fink K, Westman E, Granberg T. Repeatability and reproducibility of FreeSurfer, FSL-SIENAX and SPM brain volumetric measurements and the effect of lesion filling in multiple sclerosis. European Radiology. 2019;29(3):I355-1364. doi: I0.1007/s00330-018-57|0-x 
36. Jovicich J, Marizzoni M, Sala-Llonch R, et al. Brain morphometry reproducibility in multi-center 3T MRI studies: A comparison of cross-sectional and longitudinal segmentations. Neurolmage. 2013;83:472484. doi:I0.1016/j.neuroimage.2013.05.007

37. Verma V, Robinson CG, Rusthoven CG. Hippocampal-Sparing Radiotherapy for Patients With Glioblastoma and Grade II-III Gliomas. JAMA Oncology. Published online May 14, 2020. doi:I0.100I/ jamaoncol.2020.0164

38. Eekers DBP, Roelofs E, Cubillos-Mesías M, et al. Intensity-modulated proton therapy decreases dose to organs at risk in low-grade glioma patients: results of a multicentric in silico ROCOCO trial. Acta Oncologica. 2019;58(I):57-65. doi:10.1080/0284186X.2018.1529424

39. Grimm O, Pohlack S, Cacciaglia R, et al. Amygdalar and hippocampal volume: A comparison between manual segmentation, Freesurfer and VBM. Journal of Neuroscience Methods. 2015;253:254-261. doi:10.1016/j.jneumeth.2015.05.024

40. Khlif MS, Egorova N, Werden E, et al. A comparison of automated segmentation and manual tracing in estimating hippocampal volume in ischemic stroke and healthy control participants. Neurolmage: Clinical. 2019;21:101581. doi:10.1016/j.nicl.2018.10.019

41. Farokhian F, Beheshti I, Sone D, Matsuda H. Comparing CATI2 and VBM8 for Detecting Brain Morphological Abnormalities in Temporal Lobe Epilepsy. Frontiers in Neurology. 2017;8. doi:I0.3389/ fneur.2017.00428

42. Bernad D, Collins L, Fiocco A, et al. Analysis of Structural Changes in Hippocampal and Amygdala Volume After Systemic Therapy and Prophylactic Cranial Irradiation in Patients With Limited StageSmall Cell Lung Cancer. International Journal of Radiation Oncology*Biology*Physics. 2013;87(2):SI03. doi:10.1016/j.jijobp.2013.06.267

43. Henneghan A, Rao V, Harrison RA, et al. Cortical Brain Age from Pre-treatment to Postchemotherapy in Patients with Breast Cancer. Neurotoxicity Research. 2020;37(4):788-799. doi:10.1007/ s12640-019-00158-z 


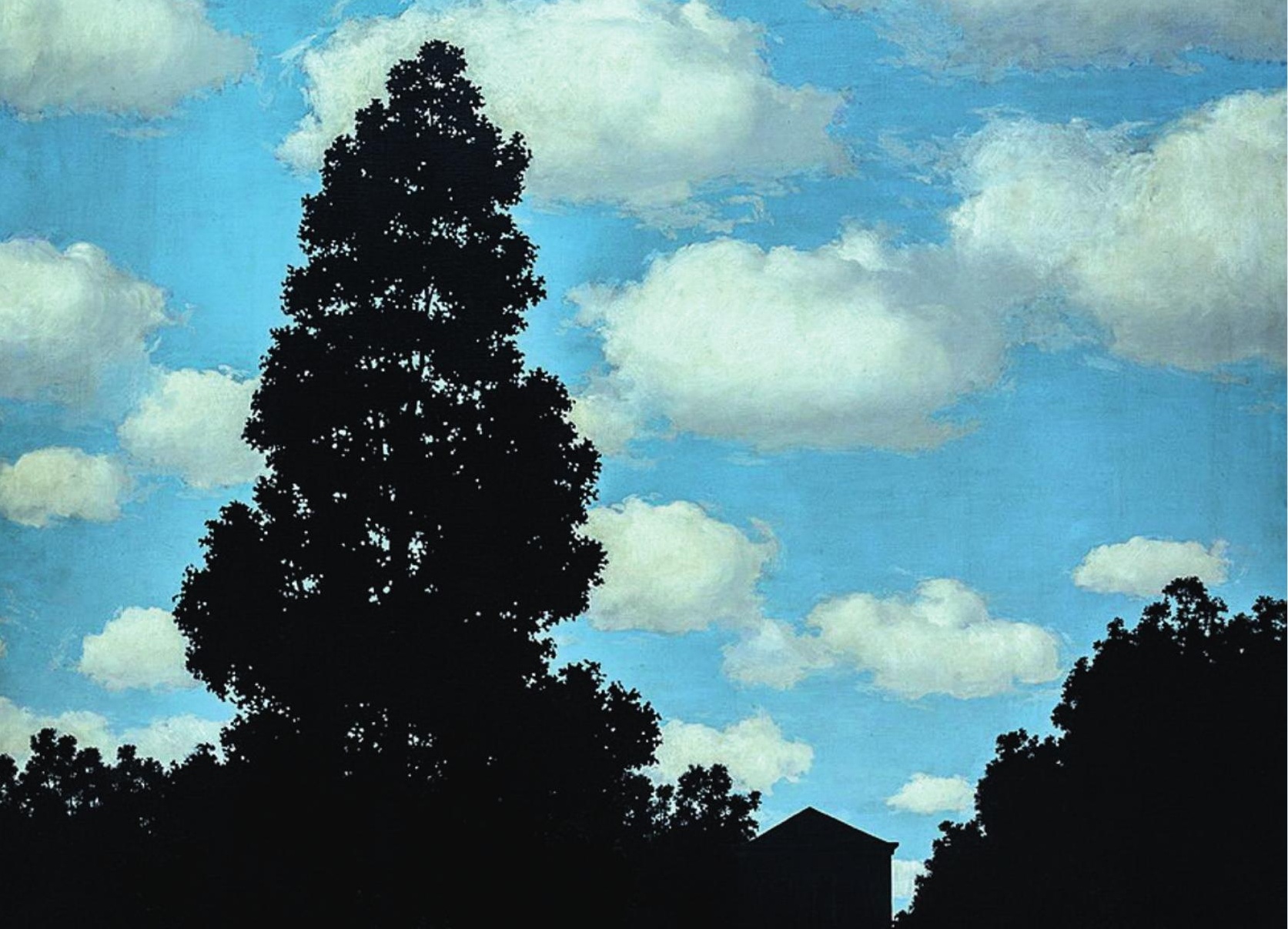

4t

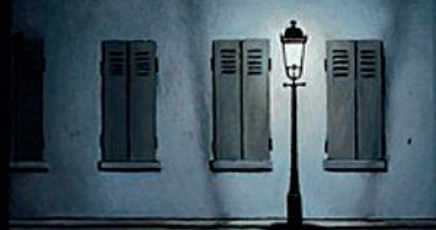




\section{Chapter 5}

Morphological changes after cranial fractionated photon radiotherapy: localized loss of white matter and grey matter volume with increasing dose

S.H.J. Nagtegaal, S. David, E.E. van Grinsven, M.J.E. van Zandvoort, E. Seravalli, T.J. Snijders, M.E.P. Philippens, J.J.C. Verhoeff 


\section{ABSTRACT}

\section{Purpose}

Numerous brain MR imaging studies have been performed to understand radiation-induced cognitive decline. However, many of them focus on a single region of interest, e.g. cerebral cortex or hippocampus. In this study, we use deformation-based morphometry (DBM) and voxel-based morphometry (VBM) to measure the morphological changes in patients receiving fractionated photon $\mathrm{RT}$, and relate these to the dose. Additionally, we study tissue specific volume changes in white matter (WM), grey matter (GM), cerebrospinal fluid and total intracranial volume (TIV).

\section{Methods and Materials}

From our database, we selected 28 patients with MRI of high quality available at baseline and I year after RT. Scans were rigidly registered to each other, and to the planning CT and dose file. We used DBM to study non-tissue-specific volumetric changes, and VBM to study volume loss in grey matter. Observed changes were then related to the applied radiation dose (EQD2). Additionally, brain tissue was segmented into WM, GM and cerebrospinal fluid, and changes in these volumes and TIV were tested.

\section{Results}

Performing DBM resulted in clusters of dose-dependent volume loss I year after RT seen throughout the brain. Both WM and GM were affected; within the latter both cerebral cortex and subcortical nuclei show volume loss. Volume loss rates ranging from 5.3 to 15.3\%/30 Gy were seen in the cerebral cortical regions in which more than $40 \%$ of voxels were affected. In VBM, similar loss rates were seen in the cortex and nuclei. The total volume of WM and GM significantly decreased with rates of $5.8 \%$ and $2.1 \%$, while TIV remained unchanged as expected.

\section{Conclusions}

Radiotherapy is associated with dose-dependent intracranial morphological changes throughout the entire brain. Therefore, we will consider to revise sparing of organs at risk based on future cognitive and neurofunctional data. 


\section{INTRODUCTION}

Radiation-induced brain injury is a phenomenon experienced after radiotherapy (RT) for brain tumors., ${ }^{1,2}$ Anatomical and functional changes can lead to cognitive impairments, ranging from mild symptoms to severe dementia-like states, and occur in 50-90\% of cases. This phenomenon is seen in patients receiving treatment for primary brain tumors, as well as those receiving whole-brain radiotherapy for brain metastases and prophylactic cranial radiotherapy.

Advances in imaging techniques have allowed the examination of the precise morphological changes in the brain after RT. Changes of white matter (WM), ${ }^{3}$ cerebral cortex, ${ }^{4-7}$ and subcortical grey matter (GM) structures ${ }^{8-10}$ have already been linked to received dose in several studies. However, these investigations have focused on specific parts or structures of the brain, which may limit the generalizability of the acquired knowledge. Techniques are available to analyze the brain in its entirety, in order to give a comprehensive estimation of the effect of RT on the brain.

One such technique is deformation-based morphometry (DBM). ${ }^{11,12}$ Here, the entire brain is analyzed, and no pre-specification of tissue type or brain region is made beforehand. Pre-RT and post-RT MRI scans are non-linearly registered to the stereotactic Montreal Neurological Institute (MNI) brain template, and the transformations applied during these steps are recorded as 3D deformation fields. These fields can be used to determine the volume changes after RT, which in turn can be related to the received dose. Investigations in epilepsy and related research have successfully applied the DBM method previously.13,14 The DBM model can be extended by adding explicit tissue segmentations. Healthy brain tissue can be segmented into WM, GM and cerebrospinal fluid (CSF). To study the GM in more detail, GM segments can be fused with 3D deformation fields in order to perform a tissue-specific investigation, often referred to as voxel-based morphometry (VBM)..$^{15}$ In VBM, the changes in each voxel between pre-RT and post-RT GM maps are measured for each patient. Next to global changes in brain tissue volume, one may also study the sum of GM, WM and CSF volumes, which together make up the total intracranial volume (TIV). ${ }^{16}$ VBM is a commonly applied tool in neuroscientific studies on aging, ${ }^{17,18}$ while TIV is more commonly used to study brain development. ${ }^{19}$

In this study, we use DBM and VBM to measure the morphological changes in glioma patients receiving fractionated photon $\mathrm{RT}$, and relate these to the dose. Additionally, we study gross volume changes in WM, GM, CSF and TIV. 


\section{METHODS}

\section{Patient selection and data collection}

We retrospectively identified scans from patients treated with RT for grade II-IV glioma at the department of Radiation Oncology in 2016 and 2017. This specific period was chosen because all glioma patients were scanned on the same MRI scanner, using the same protocols. As the MRI protocol was updated after 2017, more recently treated patients were not included to maintain data homogeneity. Patients were eligible for inclusion when the following criteria were met: treatment planning CT and MRI present, and of sufficient resolution (see below); progression free survival of at least 270 days after RT; at least I follow-up MRI between 270 days and 360 days after RT present, and of sufficient resolution.

Clinical MRI and CT scans made for RT treatment planning were extracted from patient records and anonymized, along with all follow-up MRIs, and clinical and demographic characteristics. Informed consent for this retrospective study was waived by our institutional review board.

\section{Image acquisition}

For every patient the planning CT and pre-RT MRI were collected, as well as all available follow-up MRIs. MR images were acquired on the same 3T scanner (Philips Ingenia, Philips Medical Systems, Best, The Netherlands) as part of routine clinical care. TI-weighted MR images were acquired with a 3D spoiled gradient (TFE) sequence without gadolinium enhancement with the following parameters: TR $=8.1 \mathrm{~ms}, \mathrm{TE}=3.7 \mathrm{~ms}$, flip angle $=8^{\circ}, 213$ axial slices, matrix: $207 \times 289$, voxel resolution $0.96 \times 0.96 \times 1.00 \mathrm{~mm}^{3}$. The planning CT scans were acquired on a Brilliance Big bore scanner (Philips Medical Systems, Best, The Netherlands), with a tube potential of $120 \mathrm{kVp}$, with use of a matrix size of $512 \times 512$ and $0.65 \times 0.65 \times 3.0 \mathrm{~mm}^{3}$ voxel size.

Imaging was used for three different methods: DBM, VBM and analysis of global tissue volumes (total GM, WM, CSF and TIV). For each of these methods, we analyzed the difference in volumes between baseline (pre-RT) and I year follow-up (post-RT). The latter was defined as the time point closest to 360 days after start of RT for which an MRI was available.

\section{Image processing}

Pre-RT and post-RT MRI scans were rigidly registered to each other, and to the planning $\mathrm{CT}$ and dose file. The MRI scans were processed automatically with the Computational Anatomy Toolbox (CATI2). ${ }^{20}$ First, MR images were rigidly co-registered to each other, followed by image de-noising and segmentation into GM, WM and CSF. Then, the MR 
images were nonlinearly registered to standard stereotaxic $\mathrm{MNI}$ space ${ }^{21}$ of $1.5 \mathrm{~mm}$ isotropic resolution and smoothed using an $8 \mathrm{~mm}$ kernel size. While spatial smoothing decreases the effective spatial resolution by incorporating information from neighboring voxels, it also increases the signal to noise ratio (SNR). The chosen $8 \mathrm{~mm}$ kernel presents a reasonable tradeoff and is in line with recommendations on how to perform VBM studies. ${ }^{22}$ Considering that tumor beds and the surrounding tissues show morphological changes between MRI scans not necessarily related to the applied radiation, regions covered by the planning target volume (PTV) were censored during the analysis. The PTV for grade II consisted of the enhanced area on T2-FLAIR with a margin of $1.2 \mathrm{~cm}$ (CTV+PTV margins); for grade III-IV it was the gadolinium contrast enhancing tumor on TI-weighted MRI with a margin of 2.2 $\mathrm{cm}$ (CTV+PTV margins). This step prevents errors due to tissue misclassification at the tumor bed and in the surrounding tissues, and makes sure that the observed morphological changes are related to the applied radiation.
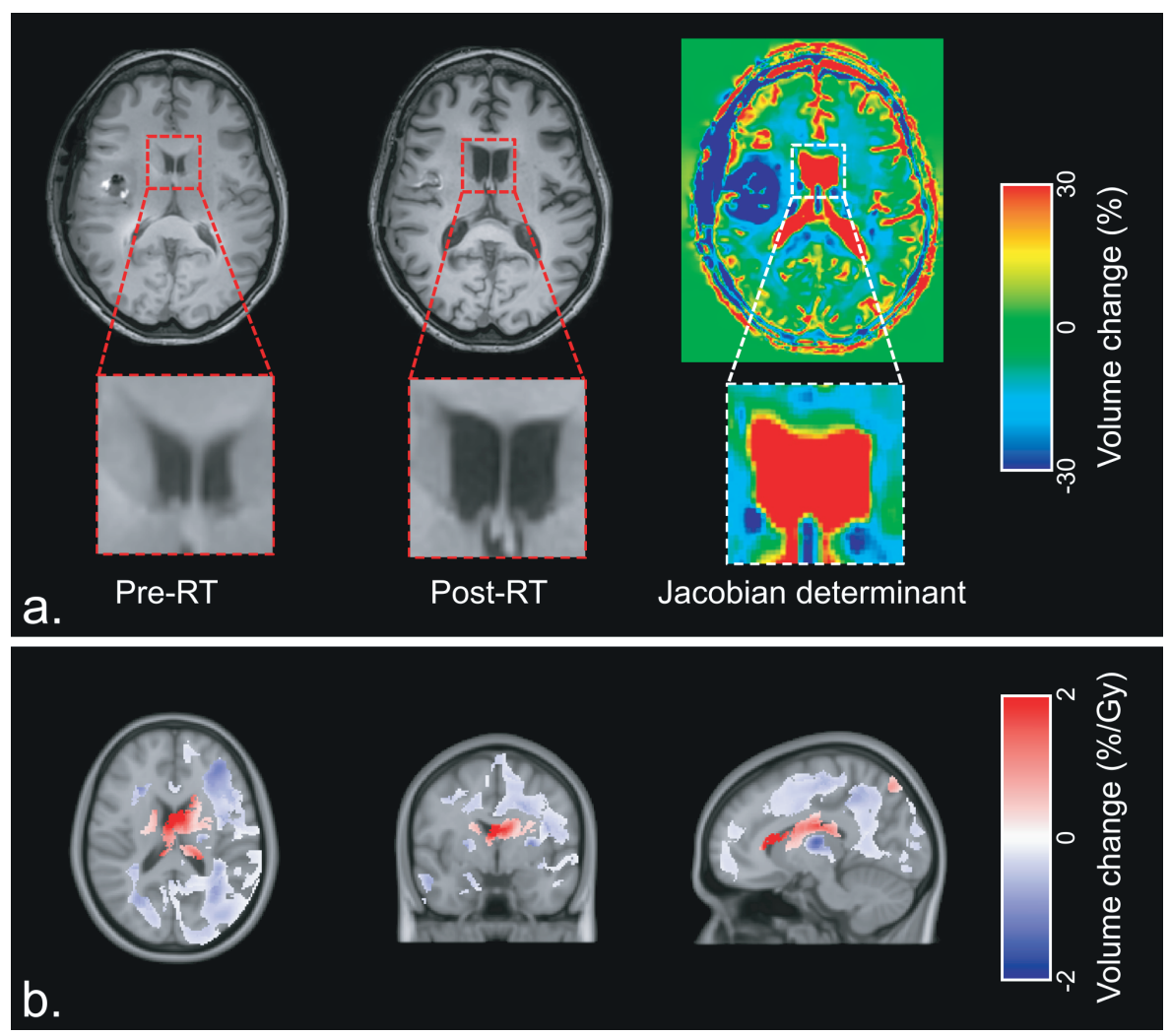

Figure I A. Example of deformation-based morphometry (DBM), in which two TI-weighted $M R$ before and after radiotherapy (RT) are used to obtain Jacobian determinants. B. Areas with significant relation between dose and volume change after I year in all patients. Blue indicates local volume loss with increasing radiation dose, red indicates volume increase. 


\section{Deformation-based morphometry (DBM)}

In DBM, the entire brain is analyzed, without pre-specifying the underlying tissue type. During non-linear registration of the individual brains to the standard template, different transformations are applied in each individual." ${ }^{112}$ This results in 3D deformation fields, in which the local volume changes (expansions or contractions) are described by the Jacobian determinants. This way, the local volumes from each scan are retained in $\mathrm{MNI}$ space. By comparing the Jacobian determinants of the pre-RT and post-RT scans, relative volumetric changes after RT were determined for each voxel (Figure IA). Then, these changes were related to the applied dose in EQD2.

\section{Voxel-based morphometry (VBM)}

In contrast to DBM, VBM is a tissue-specific analysis. ${ }^{15}$ On both pre-RT and post-RT MRI scans, GM was automatically segmented as part of the processing pipeline (Figure 2A). During VBM, the Jacobian determinants from the GM segmentations are used to investigate the modulated (volume preserved) GM changes. Relative changes in volume between the pre-RT and post-RT scans are obtained by comparing Jacobian determinants from the pre-RT and post-RT images, and then correlated to the associated MNI-warped dose maps for every patient.

\section{Statistical analysis}

Voxelwise and deformation-based statistical comparisons were carried out with a permutation test with 10,000 iterations performed with the permutation analysis of linear models (PALM) toolbox in Matlab. ${ }^{23-25}$ Significance of a correlation was determined at $\mathrm{P}_{\text {corr }}$ $<0.05$ using family-wise error rate (FWER) adjustment to correct for multiple comparisons, and 3D Threshold-Free Cluster Enhancement (TFCE) to boost the statistical power. ${ }^{26}$ Age at the time of the diagnosis and sex of the patients were included as nuisance regressors. Tail approximation was used for faster calculations. ${ }^{27}$ Significant DBM changes were expressed as relative volume changes per received radiation dose (\%/Gy), while significant VBM changes are expressed in $\mathrm{mm}^{3} / \mathrm{G}$. In order to report the brain regions in which dose-dependent volume loss occurred, we used the Neuromorphometrics brain atlas (Neuromorphometrics Inc., Somerville, Massachusetts, USA), part of the CATI2 toolbox. ${ }^{20}$ This atlas divides the brain into 142 regions (of which 126 are GM), based on anatomy and function. The atlas includes all white matter, cerebral cortex, subcortical nuclei, and the ventricles, and allows us to identify the areas that are affected by radiation. 

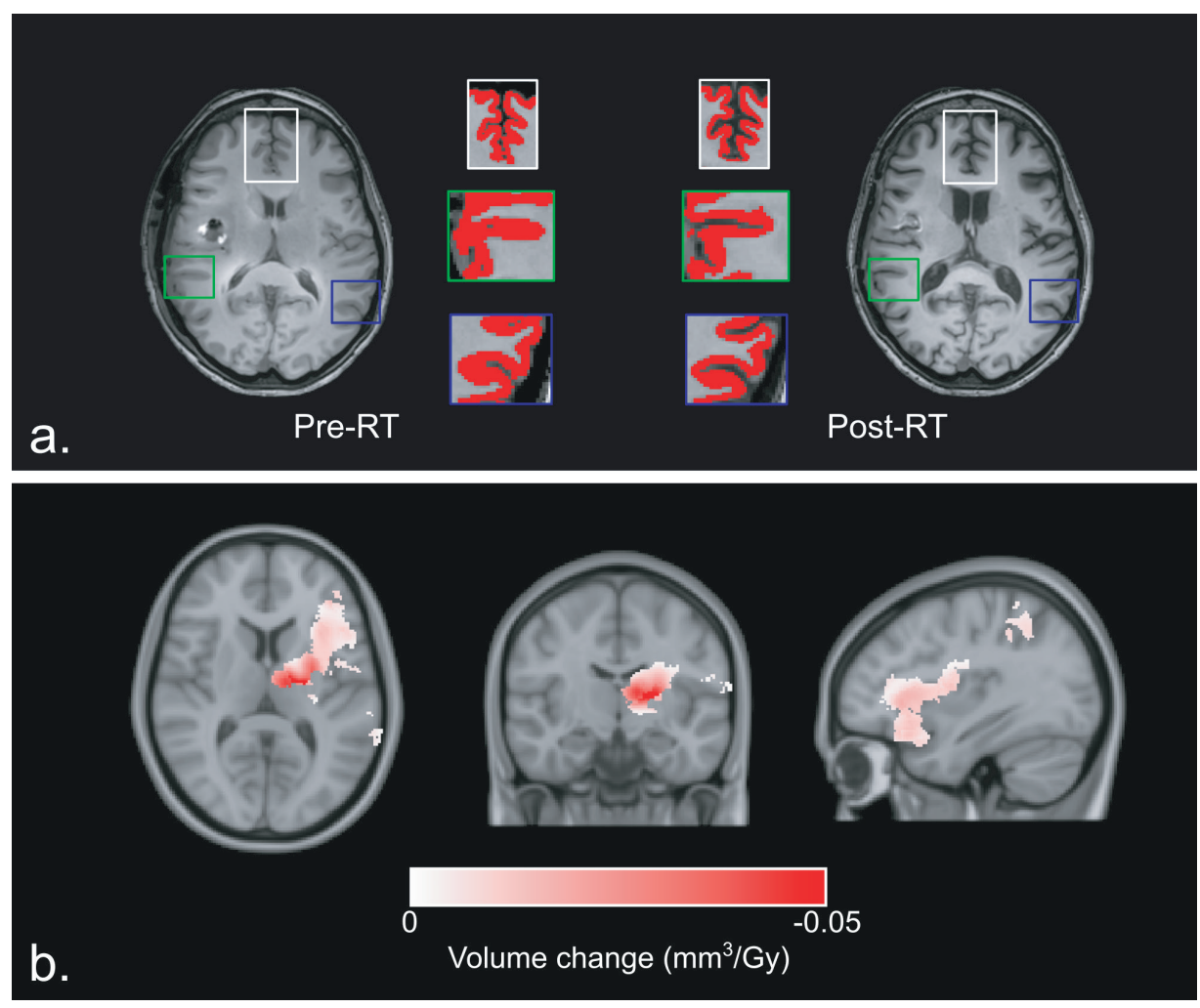

Figure 2 A. Example of grey matter (red mask) changes before and after radiotherapy (RT) as seen on TI-weighted MRI. B. Areas of significant relation between dose and volume change in all patients, as seen with voxel-based morphometry.

Differences in pre-RT and post-RT volume of GM, WM, CSF, and TIV were examined with a paired Wilcoxon signed rank test, with $p<0.05$ as the threshold of statistical significance. This was also done for the ratio between total brain tissue volume (GM+WM) and TIV.

\section{RESULTS}

\section{Participants}

Of the 170 patients who underwent RT for glioma between in 2016 and 2017, 28 fulfilled our inclusion criteria and were selected for further analysis (Supplementary Figure SI). Median age at baseline was 51 years, and $61 \%$ of patients had a high-grade glioma (Table I). All patients were treated with volumetric modulated arc therapy (VMAT). 
Table I Baseline characteristics of included patients

\begin{tabular}{ll}
\hline & N (total $\mathbf{n}=\mathbf{2 8})$ \\
\hline Age (median; IQR) & $51(38-6 \mathrm{I})$ \\
Sex & \\
Male & $17(60.7 \%)$ \\
Female & $11(39.3 \%)$ \\
WHO grade & \\
II & $11(39.3 \%)$ \\
III & $7(25 \%)$ \\
IV & $10(35.7 \%)$ \\
Tumor type & \\
Astrocytoma & $13(46.4 \%)$ \\
Oligodendroglioma & $3(10.7 \%)$ \\
Mixed & $1(3.6 \%)$ \\
Ganglioglioma & $1(3.6 \%)$ \\
Glioblastoma & $10(35.7 \%)$ \\
Prescribed dose & \\
$28 \times 1.8=50.4$ Gy & $11(39.3 \%)$ \\
$30 \times 1.8=54$ Gy & $2(7.1 \%)$ \\
$30 \times 20=60$ Gy & $15(53.6 \%)$ \\
Chemotherapy & \\
None & $4(14.3 \%)$ \\
Temozolomide & $20(71.4 \%)$ \\
PCV & $4(14.3 \%)$ \\
\hline
\end{tabular}

$\mathrm{PCV}=$ procarbazine, lomustine and vincristine

\section{DBM}

Areas of significant volume reduction with increasing dose I year after RT were seen throughout the brain as clusters of affected voxels (Figure IB). This loss of brain tissue consequently led to an increase in ventricle volume, which can be seen as clusters of voxels showing volume increase. Applying the Neuromorphometrics brain atlas revealed that, out of a total of 142 brain regions, 104 (73.2\%) contained voxel clusters showing dose-dependent volume loss. Both WM and GM were affected, and within the latter both cerebral cortex and subcortical nuclei show volume loss. Volume loss rates ranging from at least 5.3 to maximal 15.3\%/30 Gy were seen in the cerebral cortical regions in which more than $40 \%$ of voxels were affected (Supplementary Table I). In the subcortical GM the bilateral hippocampus, thalamus, putamen and globus pallidus show volume loss (Table 2) of at least $6.0 \%$ and maximal $17.5 \%$ per $30 \mathrm{~Gy}$. The left and right cerebral white matter contained clusters both showing loss rates of 11.7\%/30 Gy. Conversely, a significant lateral ventricular volume increase was observed, with a mean rate of $52.1 \% / 30$ Gy for the left and right lateral ventricle, respectively. Complete results of DBM analysis are presented in Supplementary Table 2. 
Table 2 Significant dose-dependent changes in subcortical nuclei I year after radiotherapy, as shown with deformation-based morphometry

\begin{tabular}{|c|c|c|c|c|}
\hline & & $\begin{array}{l}\text { Affected voxels } \\
\text { within area (\%) }\end{array}$ & $\begin{array}{l}\text { Relative volume } \\
\text { change }(\% / 30 \mathrm{~Gy})\end{array}$ & $\mathrm{p}$-value \\
\hline \multirow[t]{2}{*}{ Amygdala } & Left & 0 & - & - \\
\hline & Right & 20.8 & 11.8 & $<0.01$ \\
\hline \multirow[t]{2}{*}{ Caudate nucleus } & Left & 0 & - & - \\
\hline & Right & I.I & 8.6 & 0.02 \\
\hline \multirow[t]{2}{*}{ Globus pallidus } & Left & 1.3 & 8.8 & $<0.01$ \\
\hline & Right & 14.6 & 10.0 & 0.02 \\
\hline \multirow[t]{2}{*}{ Hippocampus } & Left & 21.5 & 6.0 & $<0.01$ \\
\hline & Right & 4.3 & 8.0 & $<0.01$ \\
\hline \multirow[t]{2}{*}{ Nucleus accumbens } & Left & 0 & - & - \\
\hline & Right & 0 & - & - \\
\hline \multirow[t]{2}{*}{ Putamen } & Left & 18.6 & 16.9 & $<0.01$ \\
\hline & Right & 21.8 & 12.7 & 0.02 \\
\hline \multirow[t]{2}{*}{ Thalamus } & Left & 40.5 & 17.5 & $<0.01$ \\
\hline & Right & 2.8 & 12.0 & $<0.01$ \\
\hline
\end{tabular}

\section{VBM}

Dose-dependent changes in cortical and subcortical GM volumes were observed in 42 (33.3\%) of the 126 brain atlas GM regions. Significant volume loss with increasing dose was again seen both in the cortical and subcortical GM (Figure 2B), largely as clusters of affected voxels within the left hemisphere. Of the subcortical nuclei, the left thalamus, caudate nucleus, globus pallidus and putamen contained clusters of dose-dependent volume reduction. In the seven regions in which more than $25 \%$ of voxels showed volume decrease, changes between $5.0 \%$ and $21.2 \%$ per 30 Gy were seen (Table 3). No regions showed dose-dependent increase in GM volume after RT. Complete results of VBM analysis are presented in Supplementary Table 3. 
Table 3 Brain regions in which more than 25\% of voxels show volume decrease I year after radiotherapy, as shown with voxel-based morphometry

\begin{tabular}{lllll}
\hline Region name & $\begin{array}{l}\text { Affected } \\
\text { voxels within } \\
\text { area (\%) }\end{array}$ & $\begin{array}{l}\text { Absolute volume } \\
\text { change }\left(\mathbf{m m}^{3} / \mathbf{G y}\right)\end{array}$ & $\begin{array}{l}\text { Relative } \\
\text { volume change } \\
(\% / 30 ~ G y)\end{array}$ & p-value \\
\hline Left Frontal Operculum & 61.0 & 9.4 & 16.1 & $<0.01$ \\
Left Posterior Orbital Gyrus & 48.7 & 9.2 & 13.8 & 0.01 \\
Left Anterior Insula & 47.2 & 12.3 & 12.9 & $<0.01$ \\
Left Inferior Frontal Gyrus & 35.1 & 6.0 & 6.1 & $<0.01$ \\
Left Thalamus Proper & 31.1 & 20.7 & 21.2 & $<0.01$ \\
Left Inferior Frontal Orbital & 28.9 & 2.8 & 6.1 & 0.02 \\
Gyrus & & & & $<0.01$ \\
Left Inferior Frontal Angular & 25.7 & 4.8 & 5.0 & \\
Gyrus & & & & \\
\hline
\end{tabular}

\section{Total brain volumes}

Median total brain volume (excluding the ventricles) before RT was $1131.9 \mathrm{cc}$, with a significant median decrease by 43.2 cc (3.8\%) I year after RT. Mean size and relative post-RT changes for each volume are shown in the Table 4. Significant changes are seen in all brain regions, with GM and WM showing decrease of $2.1 \%$ and $5.8 \%$, respectively. Expectedly, CSF showed an increase in volume. The total intracranial volume did not change between the two MRI scans. Additionally, the ratio of total brain tissue volume and TIV changed from a median of $80.0 \%$ to $75.7 \%(p=0.03)$ after one year.

Table 4 Changes in brain tissue volumes I year after radiotherapy

\begin{tabular}{lllll}
\hline Region & $\begin{array}{l}\text { Median pre-RT } \\
\text { volume (cc) }\end{array}$ & $\begin{array}{l}\text { Median volume } \\
\text { difference }(\mathbf{c c})\end{array}$ & $\begin{array}{l}\mathbf{9 5 \%} \mathbf{C l} \text { of } \\
\text { difference }\end{array}$ & $\mathbf{p}$ \\
\hline TIV & 1386.1 & 2.2 & $-11.0-17.6$ & 0.81 \\
GM & 608.2 & -12.8 & $-24.8--4.7$ & $<0.01$ \\
WM & 520.6 & -30.0 & $-42.0--20.1$ & $<0.01$ \\
CSF & 288.0 & 44.3 & $21.3-75.5$ & $<0.01$ \\
\hline
\end{tabular}

$\mathrm{CSF}=$ Cerebrospinal fluid; $\mathrm{GM}=$ Grey matter; $\mathrm{TIV}=$ Total intracranial volume; $\mathrm{WM}=$ White matter

\section{DISCUSSION}

We used pre-RT and post-RT MRI scans from glioma patients to assess intracranial morphological changes in the brain after fractionated photon radiotherapy. In addition 
to reports on specific susceptible areas, ${ }^{3-10}$ we found that the entire brain is susceptible to radiation induces morphological changes after $\mathrm{RT}$ for brain tumors. Total grey matter volume and total white matter volume were reduced by respectively $2.1 \%$ and $5.8 \%$, with a compensatory increase in CSF volume. The observed rate of total brain volume change of 3.8\% (combined GM and WM) is ten times higher than the normal annual atrophy rate of $0.33 \%{ }^{28}$ Deformation-based analysis of the entire brain showed volume reductions in white matter, cerebral cortex, and subcortical grey matter. The GM was further analyzed with a voxel-based analysis, again showing susceptibility in a third of cortical regions and subcortical nuclei. Finding volume changes in three distinct types of analyses strengthens the evidence for a whole brain volume-reducing effect of radiotherapy.

This is not the first study looking into the morphological changes seen after RT for brain tumors. The group of Karunami et al. and Seibert et al. ${ }^{29}$ were the first to show cortical susceptibility to dose-dependent thinning. Our recent study on this phenomenon confirmed these findings, and identified three cortical areas of heightened susceptibility, showing thinning rates after 30 Gy comparable to aging by a decade. ${ }^{5}$ Subcortical structures such as hippocampus, amygdala, have also been shown to be vulnerable to volume changes after RT. 9.10 We have repeated these analyses, and observed comparable results. ${ }^{8}$ Furthermore, we found that in addition to these two structures, the thalamus, globus pallidus, nucleus accumbens, and putamen also show an association between radiotherapy dose and post $\mathrm{RT}$ volume loss.

Connections have also been made between these morphological changes and observed cognitive decline, especially for hippocampal volume loss. Gondi et al. ${ }^{30}$ showed an association between radiation dose and memory impairments, specifically regarding delayed recall performance. The effect of radiotherapy with or without hippocampal avoidance was further studied in a phase III trial.31 Similar overall and progression-free survival were observed, but with lower risk of cognitive failure and better preservation of executive function, learning and memory.

Most of the abovementioned studies have investigated a specific part of the brain. However, the brain is a complex network of interconnected brain regions. ${ }^{32}$ This is especially crucial when considering higher order cortical functions like cognition, which have been shown to rely on large-scale neural networks. ${ }^{33}$ This means that analysis of only cerebral cortex or subcortical structures results in an incomplete picture of possible substrates of post-RT cognitive decline. We have therefore used DBM to analyze the entire brain (WM, cortex and subcortical nuclei), without prior specification of tissue type or location. This resulted in the finding that local susceptibility to radiation-induced damage is present throughout the brain. We conclude from this that a holistic approach to the discovery of etiology and 
possible prevention of cognitive decline is called for. We need to know the relation between dose and cognitive outcomes for the entire, not just for selected regions. Considering the fact that avoiding a structure like hippocampus leads to increased photon doses being delivered to other areas of the brain, ${ }^{34}$ we need to be sure these regions don't have a similar susceptibility to radiation damage.

Because we do not have neurocognitive data on the studied patients, we have to look at morphological changes in other brain diseases to understand the implications of our results. Especially in Alzheimer's disease, an association has been found between the volumes of cortical and subcortical GM and the development of cognitive impairments. ${ }^{35-39}$ Additionally, the volumetric change in at least one GM structure has been linked to cognitive abilities in normal ageing, ${ }^{40}$ Parkinson's disease, ${ }^{41-44}$ Huntington disease, ${ }^{45}$ and multiple sclerosis. ${ }^{46}$

These results may make us reconsider the currently used organs at risk (OAR) in radiation treatment of brain tumors. Several institutions have implemented hippocampal avoidance whole-brain RT to prevent cognitive decline in patients with brain metastases. However, during the treatment plan optimization, higher doses are delivered to surrounding tissue when lowering dose in a specific structure. ${ }^{47}$ We have found that this surrounding tissue can be similarly susceptible to radiation-induced damage. Future research should therefore study the relation between morphologic changes in the entire brain and cognitive outcomes. Then it can be conclusively deducted which structures should be considered OARs, and therefore should receive as little dose as possible, to limit or even prevent radiation-induced cognitive decline. Modern techniques such as VMAT and intensity modulated proton therapy (IMPT) could help us to specifically spare healthy brain tissue, should a relation between dose and cognition be found. ${ }^{48,49}$ Avoiding critical brain structures could lead to better preservation of cognition and therefore improved quality of life in brain tumors patients after treatment.

The biggest limitation to our study is the limited sample size. Due to the requirement of high quality TI MRI scans before and after RT, only a small portion of scans were eligible for inclusion. We were therefore only able to study a linear effect between the two time points. The sample size could also be the cause of the significant results of VBM to be predominantly within the left hemisphere.

However, this meticulousness improves the reliability of our results, as they are unlikely to be affected by image quality.

Secondly, RT was not the only treatment received by patients in our cohort. Most also received previous surgery and concurrent or adjuvant chemotherapy, meaning that these treatments could also have affected the observed morphological changes. However, the 
baseline scans used for analysis were made after surgery, which means surgery is less likely to have an effect on the outcome. Chemotherapy, which has been shown to cause changes in brain tissue in non-neurological malignancies, ${ }^{50,51}$ could have had a diffuse effect on brain tissue volumes. As we related the locally applied RT dose to brain morphology, we expect the role of chemotherapy to be limited.

Finally, we do not have prospectively registered neurocognitive data on the patients in our cohort. This means we cannot conclude on clinical implications of the observed morphological changes, and therefore we cannot give strong recommendations to alter current RT strategies.

\section{CONCLUSION}

To conclude, we have found that radiotherapy dose is associated with morphological changes in the entire brain. Furthermore, these changes are linked to increased dose. This may lead us to consider revising the current avoidance strategies, as now only a limited number of areas are considered organs at risk, while our data suggests the whole brain volume should be taken into account. Before this can be done, more data on the relation between these morphological changes and cognitive and other neurological outcomes after radiotherapy are needed. 


\section{REFERENCES}

I. Makale MT, McDonald CR, Hattangadi-Gluth JA, Kesari S. Mechanisms of radiotherapy-associated cognitive disability in patients with brain tumours. Nature Reviews Neurology. 2017;13(I):52-64. doi:I0.1038/nrneurol.2016.185

2. Greene-Schloesser D, Robbins ME. Radiation-induced cognitive impairment-from bench to bedside. Neuro-Oncology. 2012;14(SUPPL.4):iv37-iv44. doi:10.1093/neuonc/nos196

3. David S, Mesri HY, Bodiut VA, et al. Dose-dependent degeneration of non-cancerous brain tissue in post-radiotherapy patients: A diffusion tensor imaging study. medRxiv. Published online September 2019:19005157. doi:10.1101/19005157

4. Nagtegaal SHJ, David S, van der Boog ATJ, Leemans A, Verhoeff JJC. Changes in cortical thickness and volume after cranial radiation treatment: A systematic review. Radiotherapy and Oncology. 2019;135:3342. doi:10.1016/j.radonc.2019.02.013

5. Nagtegaal SHJ, David S, Snijders TJ, Philippens MEP, Leemans A, Verhoeff JJC. Effect of radiation therapy on cerebral cortical thickness in glioma patients: treatment-induced thinning of the healthy cortex. Neuro-Oncology Advances. 2020;2(I). doi:I0.1093/noajnl/vdaa060

6. Karunamuni R, Bartsch H, White NS, et al. Dose-Dependent Cortical Thinning After Partial Brain Irradiation in High-Grade Glioma. International journal of radiation oncology, biology, physics. 2016;94(2):297-304. doi:10.1016/j.jijrobp.2015.10.026

7. Seibert TM, Karunamuni R, Kaifi S, et al. Cerebral Cortex Regions Selectively Vulnerable to Radiation Dose-Dependent Atrophy. International Journal of Radiation Oncology Biology Physics. 2017;97(5):910918. doi:10.1016/j.jjrobp.2017.01.005

8. Nagtegaal SHJ, David S, Philippens MEP, Snijders TJ, Leemans A, Verhoeff JJC. Dose-dependent volume loss in subcortical deep grey matter structures after cranial radiotherapy. Clinical and Translational Radiation Oncology. Published online November 2020. doi:10.1016/j.ctro.2020.11.005

9. Seibert TM, Karunamuni R, Bartsch H, et al. Radiation Dose-Dependent Hippocampal Atrophy Detected With Longitudinal Volumetric Magnetic Resonance Imaging. International Journal of Radiation Oncology*Biology*Physics. 2017;97(2):263-269. doi:10.1016/j.ijrobp.2016.10.035

10. Huynh-Le M-P, Karunamuni R, Moiseenko V, et al. Dose-dependent atrophy of the amygdala after radiotherapy. Radiotherapy and Oncology. 2019;136:44-49. doi:10.1016/j.radonc.2019.03.024

II. Chung MK, Worsley KJ, Paus T, et al. A Unified Statistical Approach to Deformation-Based Morphometry. Neurolmage. 200I;14(3):595-606. doi:I0.1006/nimg.2001.0862

12. Ashburner J, Hutton C, Frackowiak R, Johnsrude I, Price C, Friston K. Identifying global anatomical differences: Deformation-based morphometry. Human Brain Mapping. 1998;6(5-6):348-357. doi:I0.1002/(SICl)I097-0193(1998)6:5/6<348::AID-HBM4>3.0.CO;2-P

13. Gaser C, Nenadic I, Buchsbaum BR, Hazlett EA, Buchsbaum MS. Deformation-based morphometry and its relation to conventional volumetry of brain lateral ventricles in MRI. Neurolmage. 2001;13(6):I|40-I|45. doi:I0.1006/nimg.2001.077|

14. Mietchen D, Gaser C. Computational morphometry for detecting changes in brain structure due to development, aging, learning, disease and evolution. Frontiers in Neuroinformatics. 2009;3(AUG). doi:10.3389/neuro.I1.025.2009

15. Ashburner J, Friston KJ. Voxel-Based Morphometry_The Methods. Neurolmage. 2000;1I (6):805-82I. doi:I0.1006/nimg.2000.0582

16. Ashburner J, Friston KJ. Unified segmentation. Neurolmage. 2005;26(3):839-85I. doi:I0.1016/j. neuroimage.2005.02.018

17. Frankea K, Dahnke R, Clarke G, et al. MRI based biomarker for brain aging in rodents and non-human primates. In: PRNI 2016 - 6th International Workshop on Pattern Recognition in Neuroimaging. Institute of Electrical and Electronics Engineers Inc.; 2016. doi:I0.II09/PRNI.2016.7552326

18. Steinke J, Gaser C, Langbein K, et al. Hippocampal metabolism and prefrontal brain structure: A combined IH-MR spectroscopy, neuropsychological, and voxel-based morphometry (VBM) study. Brain Research. 2017;1677:14-19. doi:10.1016/j.brainres.2017.09.004 
19. Zeller B, Tamnes CK, Kanellopoulos A, et al. Reduced neuroanatomic volumes in long-term survivors of childhood acute lymphoblastic leukemia. Journal of Clinical Oncology. 2013;31(17):2078-2085. doi:10.1200/JCO.20I2.47.4031

20. Gaser C, Dahnke R. CAT-a computational anatomy toolbox for the analysis of structural MRI data. Hbm. 2016;2016(7):336-348.

21. Collins DL, Neelin P, Peters TM, Evans AC. Automatic 3d intersubject registration of mr volumetric data in standardized talairach space. Journal of Computer Assisted Tomography. 1994;18(2):192-205. doi:10.1097/00004728-199403000-00005

22. Kurth F, Gaser C, Luders E. A I2-step user guide for analyzing voxel-wise gray matter asymmetries in statistical parametric mapping (SPM). Nature Protocols. 2015;10(2):293-304. doi:10.1038/nprot.2015.014

23. Winkler AM, Ridgway GR, Webster MA, Smith SM, Nichols TE. Permutation inference for the general linear model. Neurolmage. 2014:92:38I-397. doi:10.1016/j.neuroimage.2014.01.060

24. Nichols T, Holmes A. Nonparametric Permutation Tests for Functional Neuroimaging. Human Brain Function: Second Edition. 2003;25(August 1999):887-910. doi:I0.1016/B978-012264841-0/50048-2

25. Holmes AP, Blair RC, Watson JD, Ford I. Nonparametric analysis of statistic images from functional mapping experiments. Journal of cerebral blood flow and metabolism : official journal of the International Society of Cerebral Blood Flow and Metabolism. 1996;16(I):7-22. doi:10.1097/00004647-19960 I00000002

26. Smith SM, Nichols TE. Threshold-free cluster enhancement: Addressing problems of smoothing, threshold dependence and localisation in cluster inference. Neurolmage. 2009;44(I):83-98. doi:10.1016/j.neuroimage.2008.03.061

27. Winkler AM, Ridgway GR, Douaud G, Nichols TE, Smith SM. Faster permutation inference in brain imaging. Neurolmage. 2016;141:502-516. doi:10.1016/j.neuroimage.2016.05.068

28. Scahill RI, Frost C, Jenkins R, Whitwell JL, Rossor MN, Fox NC. A longitudinal study of brain volume changes in normal aging using serial registered magnetic resonance imaging. Archives of Neurology. 2003;60(7):989-994. doi:10.100I/archneur.60.7.989

29. Seibert TM, Karunamuni R, Kaifi S, et al. Cerebral Cortex Regions Selectively Vulnerable to Radiation Dose-Dependent Atrophy. International Journal of Radiation Oncology Biology Physics. 2017;97(5):910918. doi:10.1016/j.jirobp.2017.01.005

30. Gondi V, Hermann BP, Mehta MP, Tomé WA, Tome WA. Hippocampal dosimetry predicts neurocognitive function impairment after fractionated stereotactic radiotherapy for benign or lowgrade adult brain tumors. International Journal of Radiation Oncology Biology Physics. 20 I3;85(2):345-354. doi:10.1016/j.ijrobp.2012.11.031

31. Brown PD, Gondi V, Pugh S, et al. Hippocampal Avoidance During Whole-Brain Radiotherapy Plus Memantine for Patients With Brain Metastases: Phase III Trial NRG Oncology CCOOI. Journal of clinical oncology : official journal of the American Society of Clinical Oncology. Published online February 14, 2020:JCO1902767. doi:10.1200/JCO.19.02767

32. Bassett DS, Gazzaniga MS. Understanding complexity in the human brain. Trends in Cognitive Sciences. 2011;15(5):200-209. doi:10.1016/j.tics.2011.03.006

33. Bressler SL, Tognoli E. Operational principles of neurocognitive networks. International Journal of Psychophysiology. 2006;60(2):139-148. doi:10.1016/j.ijpsycho.2005.12.008

34. Verma V, Robinson CG, Rusthoven CG. Hippocampal-Sparing Radiotherapy for Patients With Glioblastoma and Grade II-III Gliomas. JAMA Oncology. Published online May 14, 2020. doi:10.100I/ jamaoncol.2020.0164

35. de Jong LW, van der Hiele K, Veer IM, et al. Strongly reduced volumes of putamen and thalamus in Alzheimer's disease: an MRI study. Brain : a journal of neurology. 2008;131(Pt 12):3277-3285. doi:10.1093/brain/awn278

36. Nie $X$, Sun $Y$, Wan $S$, et al. Subregional Structural Alterations in Hippocampus and Nucleus Accumbens Correlate with the Clinical Impairment in Patients with Alzheimer's Disease Clinical Spectrum: Parallel Combining Volume and Vertex-Based Approach. Frontiers in neurology. 2017;8:399. doi:10.3389/fneur.2017.00399 
37. Yi H-A, Möller C, Dieleman N, et al. Relation between subcortical grey matter atrophy and conversion from mild cognitive impairment to Alzheimer's disease. Journal of Neurology, Neurosurgery \& Psychiatry. 2016;87(4):425-432. doi:I0.1/36/jnnp-2014-309|05

38. Du A-T, Schuff N, Kramer JH, et al. Different regional patterns of cortical thinning in Alzheimer's disease and frontotemporal dementia. Brain. 2007;130(4):I159-1166. doi:10.1093/brain/awm0I6

39. Yin C, Li S, Zhao W, Feng J. Brain imaging of mild cognitive impairment and Alzheimer's disease. Neural regeneration research. 2013;8(5):435-444. doi:I0.3969/j.issn. 1673-5374.2013.05.007

40. Hughes EJ, Bond J, Svrckova P, et al. Regional changes in thalamic shape and volume with increasing age. Neurolmage. 2012;63(3):I I34- I I42. doi: I0. I016/j.neuroimage.2012.07.043

41. Hanganu A, Bedetti C, Degroot C, et al. Mild cognitive impairment is linked with faster rate of cortical thinning in patients with Parkinson's disease longitudinally. Brain : a journal of neurology. 2014;137(Pt 4):I120-II29. doi:10.1093/brain/awu036

42. Hünerli D, Emek-Savas DD, Çavusoglu B, Dönmez Çolakoglu B, Ada E, Yener GG. Mild cognitive impairment in Parkinson's disease is associated with decreased P300 amplitude and reduced putamen volume. Clinical Neurophysiology. 2019;130(8):1208-1217. doi:10.1016/j.clinph.2019.04.314

43. Garcia-Diaz Al, Segura B, Baggio HC, et al. Cortical thinning correlates of changes in visuospatial and visuoperceptual performance in Parkinson's disease: A 4-year follow-up. Parkinsonism \& Related Disorders. 2018:46:62-68. doi:10.1016/j.parkreldis.2017.11.003

44. Pereira JB, Ibarretxe-Bilbao N, Marti M-J, et al. Assessment of cortical degeneration in patients with Parkinson's disease by voxel-based morphometry, cortical folding, and cortical thickness. Human Brain Mapping. 2012;33(II):2521-2534. doi:10.1002/hbm.21378

45. Aylward EH, Harrington DL, Mills JA, et al. Regional atrophy associated with cognitive and motor function in prodromal Huntington disease. Journal of Huntington's disease. 2013;2(4):477-489. doi:10.3233/JHD-130076

46. Rojas JI, Murphy G, Sanchez F, et al. Thalamus volume change and cognitive impairment in early relapsing-remitting multiple sclerosis patients. The neuroradiology journal. 2018;3I(4):350-355. doi:10.1 177/1971400918781977

47. Verma V, Robinson CG, Rusthoven CG. Hippocampal-Sparing Radiotherapy for Patients With Glioblastoma and Grade II-III Gliomas. JAMA Oncology. Published online May 14, 2020. doi:10.100I/ jamaoncol.2020.0164

48. Grosshans DR, Mohan R, Gondi V, Shih HA, Mahajan A, Brown PD. The role of image-guided intensity modulated proton therapy in glioma. Neuro-Oncology. 2017;19(suppl_2):ii30-ii37. doi:I0.1093/neuond nox002

49. Sood S, Pokhrel D, McClinton C, et al. Volumetric-modulated arc therapy (VMAT) for whole brain radiotherapy: not only for hippocampal sparing, but also for reduction of dose to organs at risk. Medical Dosimetry. 2017;42(4):375-383. doi:10.1016/j.meddos.2017.07.005

50. Bernad D, Collins L, Fiocco A, et al. Analysis of Structural Changes in Hippocampal and Amygdala Volume After Systemic Therapy and Prophylactic Cranial Irradiation in Patients With Limited StageSmall Cell Lung Cancer. International Journal of Radiation Oncology*Biology*Physics. 2013;87(2):SI03. doi:10.1016/j.jijrobp.2013.06.267

51. Henneghan A, Rao V, Harrison RA, et al. Cortical Brain Age from Pre-treatment to Postchemotherapy in Patients with Breast Cancer. Neurotoxicity Research. 2020;37(4):788-799. doi:10.1007/ s|2640-019-00158-z 
Morphological changes in grey and white matter after radiotherapy 


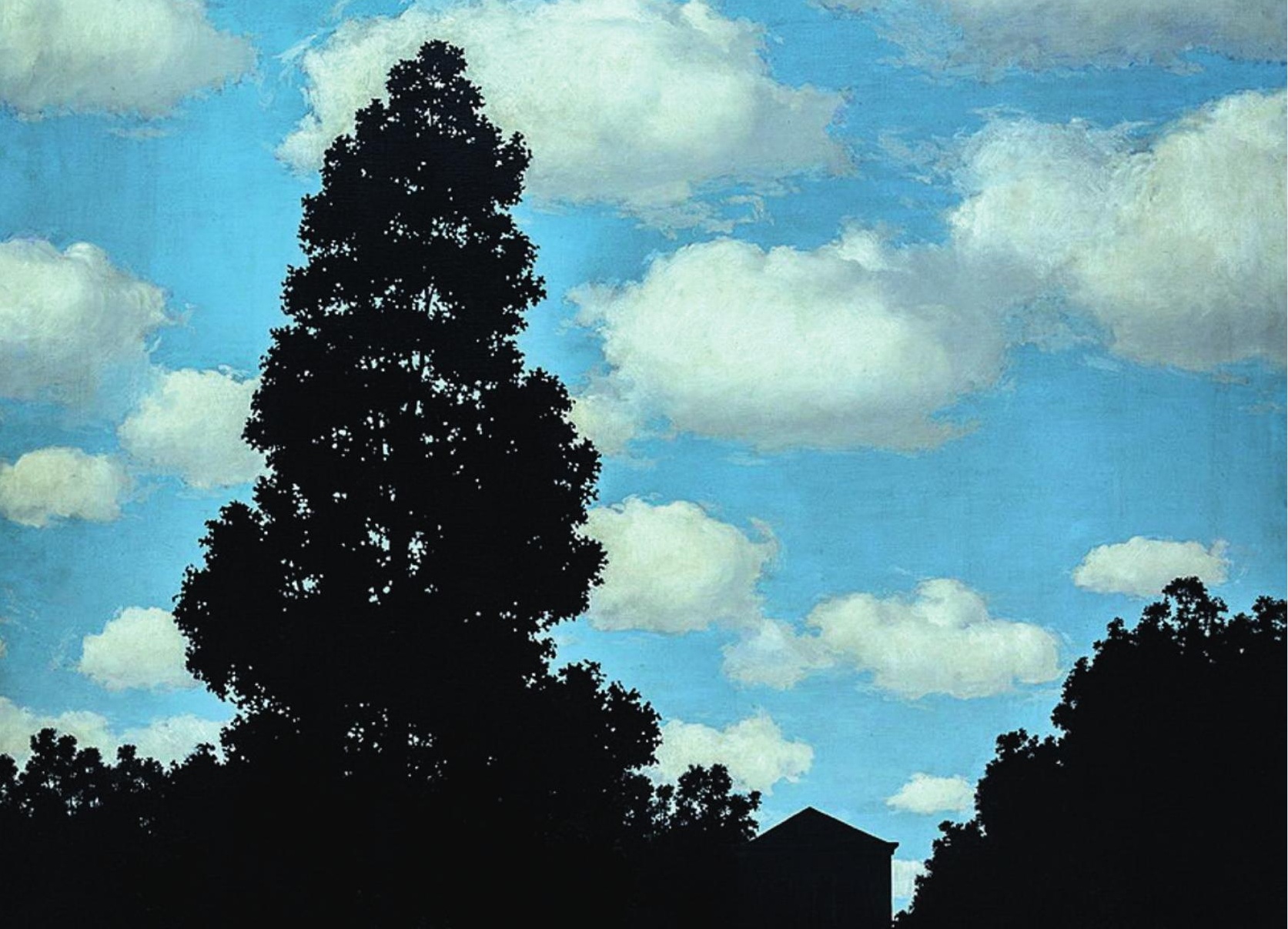

4t

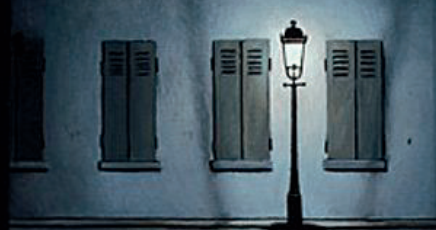




\section{Chapter 6}

Comparing survival predicted by the diagnosis-specific Graded Prognostic Assessment (DS-GPA) to actual survival in patients with I- 10 brain metastases treated with stereotactic radiosurgery

S.H.J. Nagtegaal, A. Claes, K.P.M. Suijkerbuijk, F.M.N.H. Schramel, T.J. Snijders, J.J.C. Verhoeff 


\section{ABSTRACT}

\section{Background and purpose}

Multiple prognostic models for predicting survival after treatment for brain metastases have been developed. One of them, the diagnosis-specific Graded Prognostic Assessment (DS-GPA), has been developed to predict the median survival for brain metastases from the most frequent primary sites: lung carcinoma, breast cancer, melanoma, renal cell cancer and gastrointestinal tumours. In this study we aim to compare the survival predicted by the DS-GPA to actual survival, and to assess this model's performance on both population and individual levels.

\section{Methods}

We identified a consecutive cohort of patients treated with SRS for brain metastases in our institute. DS-GPA scores were calculated for each patient, and the median survival for each DS-GPA group was calculated. Differences in survival between DS-GPA groups were tested with Kaplan-Meier curves and log-rank tests.

\section{Results}

In total 367 patients were included in analysis. Median survival in our cohort is largely comparable to corresponding DS-GPA cohorts, but some notable differences are present. There was a significantly shorter median survival (I5.4 months, compared to 26.5 months) in the adenocarcinoma NSCLC subgroup with a GPA score of 2.3-3. We confirmed the significant differences in survival time for most cancer-specific subgroups.

\section{Conclusion}

DS-GPA seems to be a reliable tool to classify patients with brain metastases treated with SRS into prognostic subgroups. However, we found some aberrations from predicted median survival times, which may be due to specific characteristics of the populations of patients treated with SRS versus other patients. 


\section{INTRODUCTION}

Of all patients diagnosed with cancer, 8-10\% will develop brain metastases (BM).' This incidence is expected to increase with more effective treatments for the primary tumours, thereby improving survival and thus increasing the time for possible dissemination of tumour to the brain. ${ }^{1,2}$ Brain metastases most frequently originate from lung cancer, breast cancer and melanoma, ${ }^{1-5}$ but in up to $14 \%$ of patients, the metastases are of unknown origin. ${ }^{3-5}$

The expected survival is an important factor in selecting the most optimal treatment. To aid this process, several scores have been developed to predict survival of patients with brain metastases. One of them, the diagnosis-specific Graded Prognostic Assessment (DSGPA), has a specific predictive model for the five most prevalent primary sites: lung cancer, breast cancer, melanoma, renal cell carcinoma (RCC) and gastro-intestinal (GI) cancer. ${ }^{6}$ Each model gives the estimated median survival and its IQR based on several criteria, among which Karnofsky Performance Score (KPS), age, number of brain metastases, presence of extra-cranial metastases and disease-specific tumour markers. These predictive models are constantly being updated with the newest findings and additional patient data. The scores for lung cancer and melanoma now feature the molecular subtypes of the tumour, and the model for RCC has recently been updated to include haemoglobin count $(\mathrm{Hb})$ at baseline. ${ }^{7-9}$ The most recent models are freely available on brainmetgpa.com, ${ }^{10}$ and can be used by physicians and patients alike.

Over the last few years, an increasing proportion of BMs are being treated with stereotactic radiosurgery (SRS). In the Netherlands, the current guidelines suggest treating patients with I-3 BMs with SRS," but observational studies in which patients with four or more lesions were treated suggest that SRS is also a valid treatment for these patients. ${ }^{12,13}$ There are even centres where patients with $\geq 10$ or $\geq 15$ BMs are treated with SRS only.14,15

This change in treatment protocols may lead to a different survival, and so may lead to inaccurate estimations of the median survival with the DS-GPA. Therefore, we retrospectively computed the DS-GPA for patients with brain metastases who were treated with SRS in our centre, in order to compare the predicted survival to actual survival. The aim of this study is twofold: I) testing to what extent the DS-GPA is able to stratify our patient cohort into groups with different survival, and 2) how well the DS-GPA is able to predict the survival for individual patients. 


\section{METHODS}

\section{Patient selection}

We retrospectively identified a cohort of consecutive patients treated with SRS for brain metastases in the University Medical Center in Utrecht, the Netherlands between January 2012 and July 2017. Patients were eligible if they were treated with SRS for newly discovered brain metastases within that period, and when their primary tumour was one of the following: non-small cell lung carcinoma (NSCLC), breast cancer, melanoma, RCC or GI cancer. Even though the lung-specific GPA model still applies to SCLC patients according to the brainmetgpa.com website, ${ }^{10}$ no SCLC cases were included in this study. The lungmolGPA was based solely on NSCLC patients, therefore only these patients were used to assess this model. ${ }^{8}$

As this retrospective study only involves patient files, the need for informed consent was waived. We obtained permission from the Ethics Board of our institution to conduct this study. This study was done according to the Code of Conduct for Medical Research as set up by the Dutch Federation of Biomedical Scientific Societies.

\section{Data collection}

Baseline data were collected from patient records. Collected data consisted of patient demographics, primary tumour and metastasis characteristics and survival. Additionally, data needed for DS-GPA calculation were collected: KPS, BRAF gene status (for melanoma), EGFR and ALK gene status (for NSCLC), Hb (converted from mmol/l to g/dL, for RCC) and Her2, ER and PR receptor status (for breast cancer).

\section{Outcome}

For calculation of the DS-GPA, the most current scoring method and predicted median survival were used (See Box I). For NSCLC and melanoma, the scores that incorporated molecular markers (Lung-molGPA and Melanoma-molGPA) were used. ${ }^{8,9}$ For breast cancer and GI tumours, the DS-GPA scoring was taken from the latest summary on DS-GPA. ${ }^{6}$ The newest model for calculating DS-GPA for RCC was taken from a recent update, which incorporates $\mathrm{Hb}$ at baseline as a criterion. ${ }^{7}$ In order to avoid confusion, all the aforementioned scores will be called "DS-GPA" in this publication.

For each patient, the disease-specific GPA score was calculated with the collected clinical data. In case of any missing data crucial for DS-GPA (i.e. where "unknown" was not in the scoring model), no DS-GPA was calculated. These patients were not excluded from further analysis, in order to give an accurate portrayal of our patient cohort. 
Box I: DS-GPA scores for each tumour subtype

\begin{tabular}{|c|c|c|c|c|c|}
\hline NSCLC & 0 & 0.5 & 1 & & \\
\hline Age & $\geq 70$ & $<70$ & - & & \\
\hline KPS & $<70$ & 80 & $90-100$ & & \\
\hline ECM & Present & - & Absent & & \\
\hline BM number & $\geq 5$ & $1-4$ & - & & \\
\hline Gene status & $\begin{array}{c}\text { EGFR neg/ } \\
\text { unk and } \\
\text { ALK neg/ } \\
\text { unk }\end{array}$ & - & $\begin{array}{l}\text { EGFR pos } \\
\text { or ALK pos }\end{array}$ & & \\
\hline Breast cancer & 0 & 0.5 & I & 1.5 & 2 \\
\hline Age & $\geq 60$ & $<60$ & - & - & - \\
\hline KPS & $\leq 50$ & 60 & $70-80$ & $90-100$ & - \\
\hline Subtype* & Basal & - & LumA & HER2 & LumB \\
\hline Melanoma & 0 & 0.5 & I & & \\
\hline Age & $\geq 70$ & $<70$ & - & & \\
\hline KPS & $\leq 70$ & 80 & $90-100$ & & \\
\hline ECM & Present & - & Absent & & \\
\hline BM number & $\geq 5$ & $2-4$ & । & & \\
\hline BRAF gene status & Neg/unk & Pos & - & & \\
\hline $\mathrm{RCC}$ & 0 & 0.5 & I & 2 & \\
\hline KPS & $\leq 70$ & - & 80 & $90-100$ & \\
\hline ECM & Present & Absent & - & - & \\
\hline $\mathrm{Hb}(\mathrm{g} / \mathrm{dL})$ & $\leq \mid 1.1$ & $\begin{array}{c}\text { 11.2-12.5 or } \\
\text { unk }\end{array}$ & $\geq 12.6$ & - & \\
\hline BM number & $\geq 5$ & $1-4$ & - & - & \\
\hline GI tumours & 0 & I & 2 & 3 & 4 \\
\hline KPS & $\leq 60$ & 70 & 80 & 90 & 100 \\
\hline
\end{tabular}

*Subtype definitions: Basal: triple negative; LumA: ER/PR positive, HER2 negative; LumB: triple positive; HER2: ER/PR negative, HER2 positive

ALK = Anaplastic lymphoma kinase, BM = brain metastasis, ECM = extracranial metastases, EGFR $=$ Epidermal growth factor receptor, $E R=$ estrogen receptor, $H b=$ haemoglobin, HER2 = human epidermal growth factor receptor 2, KPS = Karnofsky Performance Score, Neg = negative, NSCLC=non-small cell lung carcinoma, Pos = positive, PR = progesterone receptor, Unk = unknown 
Survival was defined as the time between first treatment for brain metastasis and death, or the time between first treatment and date of censoring in case patients were still alive. This definition was also used in creating the DS-GPA. ${ }^{6-9}$ Patients without recorded death in our patient files were checked in the Municipal Personal Records Database (Gemeentelijke Basisadministratie, GBA) on July $17^{\text {th }} 2018$, in order to verify whether they were still alive.

\section{Statistical analysis}

Descriptive baseline data were calculated. The mean survival with its interquartile range (IQR) was calculated, stratified per tumour type and DS-GPA score. A one-sample Wilcoxon Signed Rank test was performed to test for significant differences in actual and predicted median survival. We used the Bonferroni correction to correct for multiple testing with an adjusted $p$-value threshold of 0.002 (0.05/21). Additionally, survival was marked as being within or outside the predicted IQR, in order to test whether half of the survival was within this range. Kaplan-Meier curves were created for each disease group stratified by DS-GPA score, with use of the log-rank test to test for significant differences between the groups. The significance threshold was set at 0.05 .

All analyses were performed with SPSS Statistics for Windows, Version 23.0 (IBM Corp., Armonk, NY, USA).

\section{RESULTS}

\section{Participants}

In total, 40I patients who were treated with SRS for newly discovered brain metastasis were identified. Of them, 367 had either NSCLC, melanoma, breast cancer, RCC or GI tumour as primary malignancy, and were included in further analysis. Baseline characteristics of these patients are presented in Table I. In accordance with Dutch national guidelines, SRS was considered first-choice treatment in patients with no more than 3 brain metastases. On an individual basis, the tumour board advised SRS for patients with more brain metastases (mostly 4-6), depending on their DS-GPA life expectancy of more than 3 months and systemic therapy options. The dose of SRS is according to Dutch national guidelines $(<\mid c c$ 24Gy, I-10cc 21Gy, 10-20cc 18Gy, 20-65cc 15Gy).

\section{DS-GPA scores}

Calculated DS-GPA scores per tumour group are presented in Supplementary Table I.

DS-GPA could not be calculated for one breast cancer patient ( $0.3 \%$ of all patients) due to missing receptor status. 


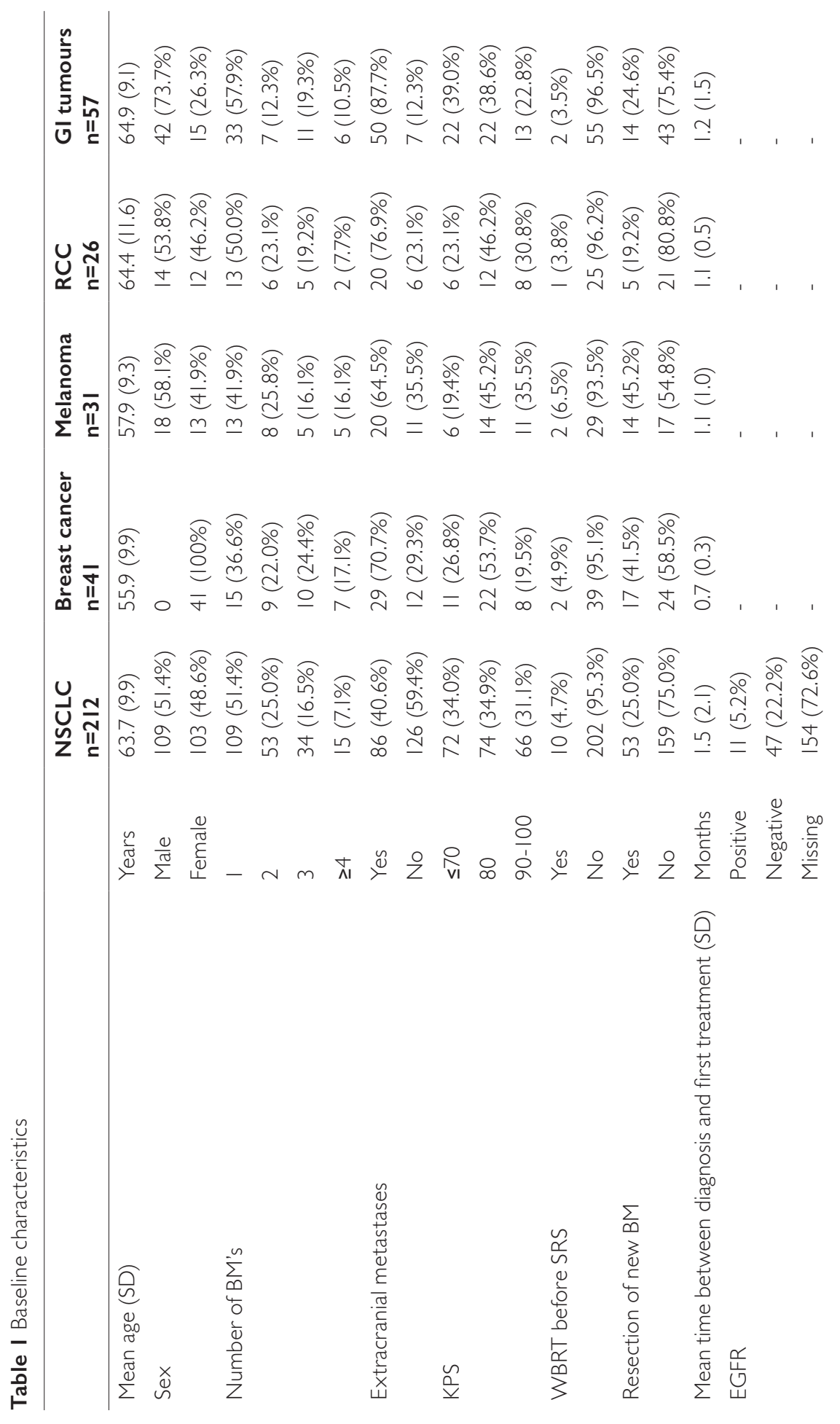




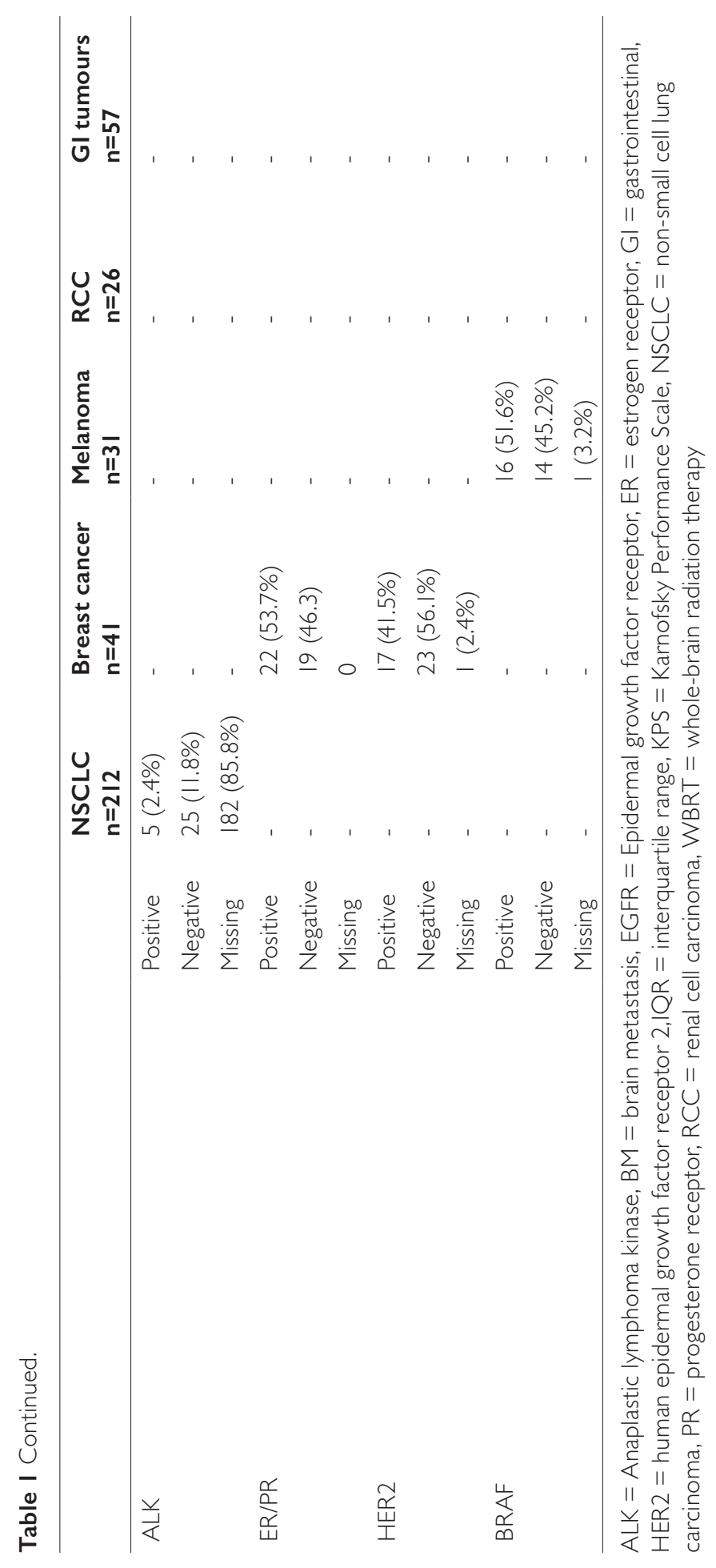


Table 2 DS-GPA scores and median survival in our patient cohort, compared to survival as predicted by DS-GPA

\begin{tabular}{|c|c|c|c|c|c|}
\hline Primary tumour type & $\begin{array}{l}\text { DS-GPA } \\
\text { score }\end{array}$ & $n$ & $\begin{array}{l}\text { Median survival } \\
\text { in our patient } \\
\text { cohort (IQR) }\end{array}$ & $\begin{array}{l}\text { Median survival } \\
\text { as predicted by } \\
\text { DS-GPA (IQR) }\end{array}$ & $\mathrm{p} \dagger$ \\
\hline NSCLC & $0-1$ & 21 & $7.7(2.6-18.3)$ & $6.9(2.6-15.3)$ & 0.114 \\
\hline \multirow[t]{3}{*}{ Adenocarcinoma* } & $1.5-2$ & 71 & ||.2(5.3- |8.3) & $13.7(5.5-24.6)$ & 0.112 \\
\hline & $2.5-3$ & 43 & $15.4(9.3-27.4)$ & $26.5(10.7-53.8)$ & $0.001+t$ \\
\hline & $3.5-4$ & 3 & $29.5(25.6-35.6)$ & $46.8(25.8-)$ & 0.068 \\
\hline NSCLC & $0-1$ & 7 & $2.6(1.4-3.4)$ & $5.3(1.9-11.1)$ & 0.237 \\
\hline \multirow[t]{2}{*}{ Non-adenocarcinoma* } & $1.5-2$ & 24 & $6.1(2.1-7.3)$ & $9.8(3.9-20.3)$ & 0.011 \\
\hline & $2.5-3$ & 18 & $8.9(5.2-18.5)$ & $12.8(7.0-30.1)$ & 0.586 \\
\hline \multirow[t]{5}{*}{ Breast cancer } & $0-1$ & 2 & $11.4(2.0-)$ & $3.4(1.4-7.3)$ & 0.655 \\
\hline & $1.5-2$ & 16 & $7.1(4.3-38.8)$ & $7.7(3.0-15.2)$ & 0.234 \\
\hline & $2.5-3$ & 16 & $18.5(10.9-27.7)$ & I5.| (5.9- 27.4) & 0.134 \\
\hline & $3.5-4$ & 6 & $16.3(13.9-23.7)$ & $25.3(12.8-45.8)$ & 0.345 \\
\hline & No GPA*** & । & 6.0 & - & \\
\hline \multirow[t]{4}{*}{ Melanoma } & $0-1$ & 2 & $11.5(1.9-)$ & $4.9(2.3-10.7)$ & 0.655 \\
\hline & I.5-2 & 15 & $7.8(4.2-15.5)$ & $8.3(3.9-18.2)$ & 0.496 \\
\hline & $2.5-3$ & 8 & $22.9(16.9-34.9)$ & $15.8(8.2-49.3)$ & 0.069 \\
\hline & $3.5-4$ & 6 & $24.0(12.4-51.7)$ & 34.1 (I5.I-) & 0.600 \\
\hline \multirow[t]{4}{*}{$\mathrm{RCC}$} & $0-1$ & 3 & $4.4(1.9-)$ & $4(2-8)$ & 0.593 \\
\hline & $1.5-2$ & 8 & II.8 (3.0-22.I) & $12(5-24)$ & 1.000 \\
\hline & $2.5-3$ & 10 & $27.6(14.8-47.7)$ & $17(8-36)$ & 0.059 \\
\hline & $3.5-4$ & 5 & $8.7(5.0-17.3)$ & $35(|3-6|)$ & 0.043 \\
\hline \multirow[t]{4}{*}{ Gl tumour } & $0-1$ & 22 & $4.3(1.8-8.6)$ & $3.1(1.8-6.2)$ & 0.039 \\
\hline & $1.5-2$ & 22 & $7.5(1.7-11.9)$ & $4.4(2.4-10.4)$ & 0.020 \\
\hline & $2.5-3$ & 8 & $11.7(3.6-17.1)$ & $6.9(4.1-15.2)$ & 0.161 \\
\hline & $3.5-4$ & 5 & $7.6(4.1-26.9)$ & $13.5(9.9-27.1)$ & 0.686 \\
\hline
\end{tabular}

* NSCLC subtype unknown for 24 patients

*** No GPA calculable due to missing tumour receptor status

† Bold values signify those under the unadjusted significance threshold of 0.05

$+\uparrow$ Value under adjusted significance threshold of 0.002

DS-GPA=disease-specific graded prognostic assessment, GI = gastrointestinal,

$\mathrm{IQR}=$ interquartile range, NSCLC = non-small cell lung carcinoma, $\mathrm{RCC}=$ renal cell carcinoma

\section{Survival}

Overall, the median survival was 10.5 months, with a predicted median survival of 13.5 months. Median survival per tumour group and DS-GPA score is presented in Table 2, together with the predicted median survival and the results of the median test. In the 
NSCLC subgroups, 24 patients could not be included in median survival testing due to missing NSCLC tumour type. After correcting for multiple testing, a significant difference was only found the 2.5-3.0 DS-GPA strata in the adenocarcinoma NSCLC subgroup, in which the median survival in our cohort was 10.5 months shorter than predicted (I5.4 months, compared to 26.5 months).

In the entire patient cohort, $55.6 \%$ of survival times were within the predicted IQR. Survival below QI was seen in 26.6\%, and $17.8 \%$ lived longer than the predicted third quartile. Similarly, $42.9 \%$ of patients lived longer than the predicted median survival.

Waterfall plots showing the difference between the predicted median and the actual survival on a per patient basis, stratified by number of BMs are shown in Figure I.

Kaplan-Meier curves per tumour group and DS-GPA score are shown in Figure 2. A significant difference in survival between patients with different DS-GPA scores was seen in the adenocarcinoma NSCLC, non-adenoma NSCLC, melanoma and RCC subgroups. In the patients with breast cancer or GI cancer, no significant differences were found.

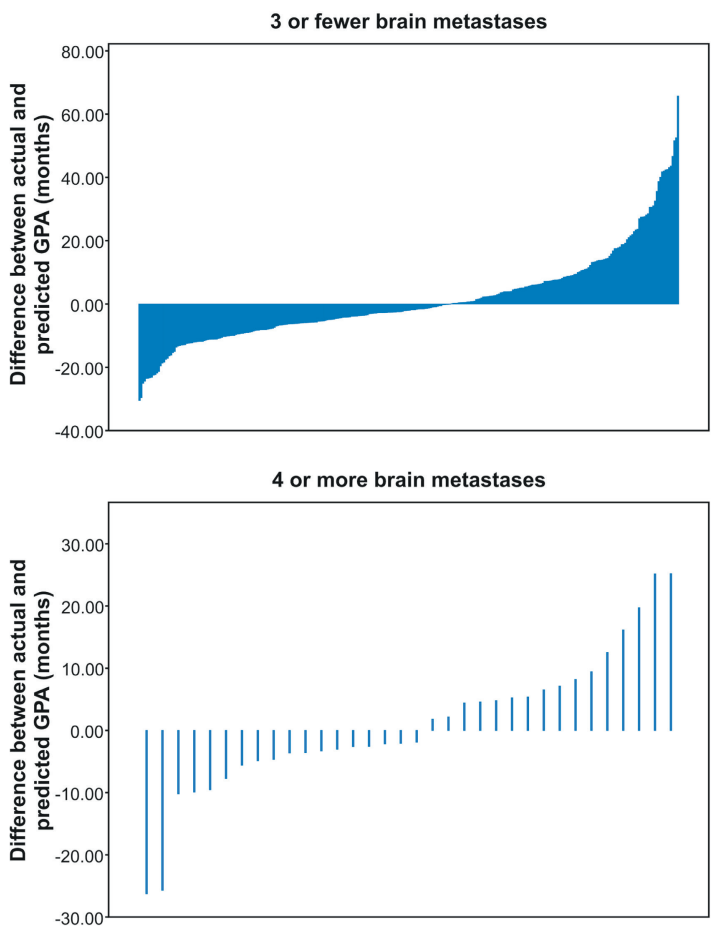

Figure I Waterfall plots showing the differences between the predicted and the actual survival on an individual level, stratified by the number of brain metastases. 

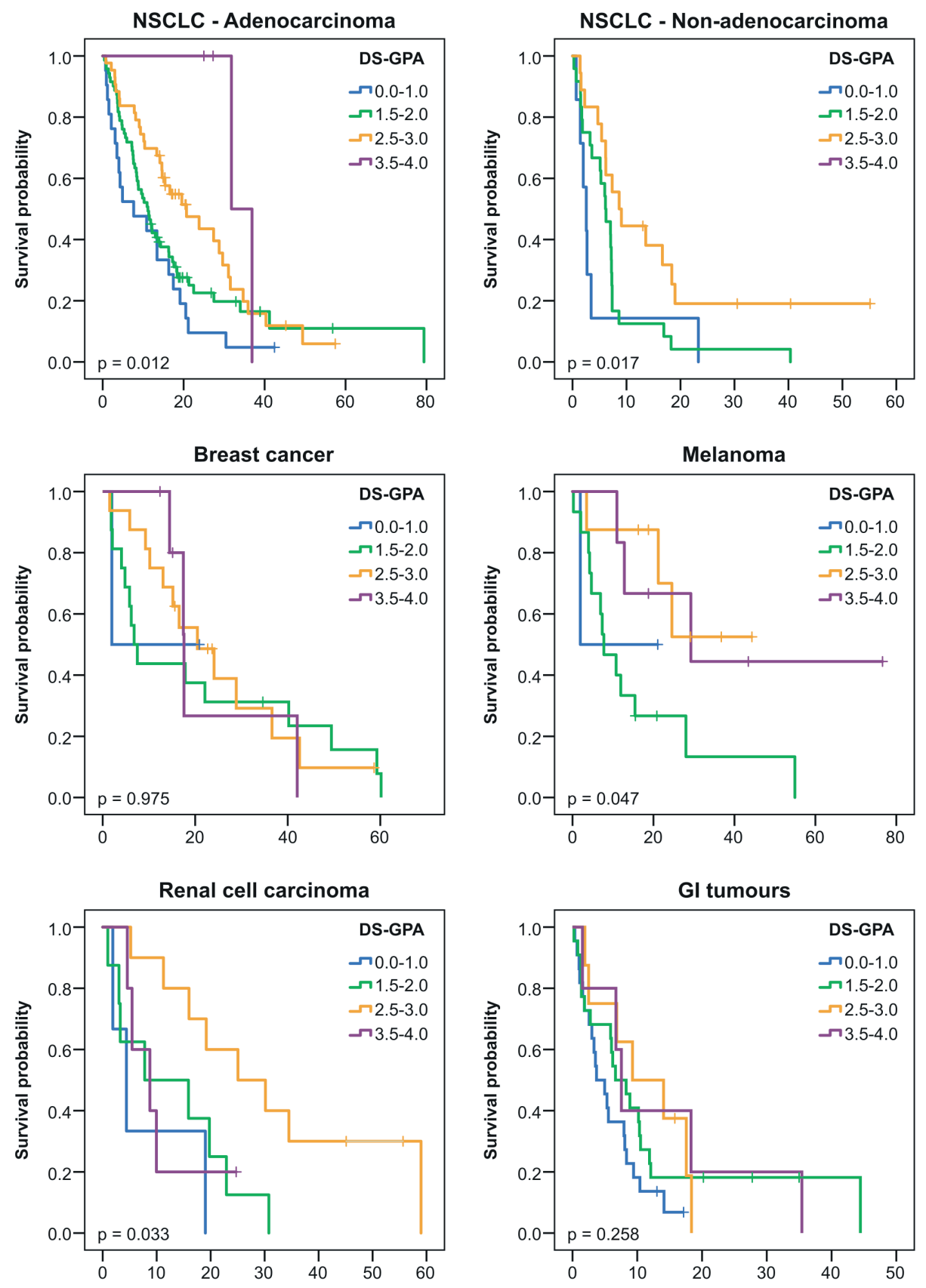

Figure 2 Survival curves per DS-GPA-subgroup for each cancer type. P-values from log-rank tests are given, revealing significant differences between the DS-GPA strata in four disease subgroups. In these subgroups, the DS-GPA works for stratifying by survival. 
In 30 patients (8.2\%), the interval between MRI diagnosis and first intracranial treatment exceeded 2 months. Mostly administration of systemic therapy caused this planned delay (as concurrent systemic therapy and RT is discouraged in our guidelines" ${ }^{\prime \prime}$ ), although for some patients a "wait-and-scan" protocol was decided on. As these patients were excluded in Sperduto's patient cohorts used for creating the scoring models, a post-hoc analysis was performed with these patients excluded from analysis. The differences with the original analysis were minor: a new significant difference in median survival was seen for the RCC subgroup with DS-GPA scores of 2.5-3.0 (median survival 30.2 months, vs 17 months predicted, $p=0.018$ by log-rank test). Inversely, the log-rank test lost significance in the non-adenocarcinoma NSCLC group.

\section{DISCUSSION}

There are two ways a scoring method such as the DS-GPA can be interpreted. Firstly, its prediction can be seen as an estimate of the expected survival on an individual level, which aids the physician to give an accurate estimation of prognosis to the patient. Secondly, it is used as a tool to stratify the patient population into groups with a more or less favourable survival, which may help to decide on the most suitable treatment and intensity of follow-up. We have noticed that the two ways of interpreting the DS-GPA results are used in clinical practise, while only the latter one is the formal intention of the model. Therefore, we have tested the performance of the DS-GPA for these two applications in patients with brain metastases who were treated with SRS.

For the first application, the DS-GPA seems to be a valuable tool. Although the waterfall plots reveal some large differences between the actual and predicted median survival, especially in patients with longer survival, we found that around half of the patients reached the predicted median survival. Similarly, more than half of the patients' survival fell within the predicted IQR. This means that the median survival (and the IQR) from the DS-GPA model is an accurate prediction of the actual survival in our cohort from clinical practice. It also confirms that the DS-GPA does not provide a point prediction of survival on an individual level.

On a group basis, our results prove to be less conclusive. Although most DS-GPA strata in the disease subgroups showed no significant difference in median survival, one stratum of patients with adenocarcinoma NSCLC had a 10 month shorter median survival than predicted, which remained significant even after correcting for multiple comparisons. Kaplan-Meier curves revealed that DS-GPA was useful for dividing the NSCLC, melanoma and RCC subgroups into strata with significantly different survival times. The other disease 
subgroups showed no significant difference in survival between the DS-GPA strata, although this may be due to insufficient power in these smaller subgroups.

There are several possible explanations for any of the differences found between predicted and actual survival. Firstly, this cohort only included patients treated with SRS, whereas Sperduto et al.'s patient populations underwent a variety of treatments (WBRT, SRS, surgery, or a combination of the three). ${ }^{6-9}$ In the databases used to create the models for RCC, breast and GI tumours, 34-61\% of patients did not receive SRS, but received only WBRT and/or surgery instead. In the melanoma and lung databases, these proportions are 15\% and 5\%, respectively. According to the current Dutch guidelines, only patients with certain characteristics ( $\leq 3 \mathrm{BMs}$ and KPS $\geq 70$ ) should be considered for SRS, which means this cohort consists of a subset of patients that does not represent the entirety of the BM patient population. Although our analysis, comparing DS-GPA-strata, automatically corrects for some of these baseline differences, some residual differences between cohorts may exist.

Another possible problem with a prediction model is misclassification of patients. This problem is unlikely to occur as a result of objectively measured criteria, which the DS-GPA criteria are with the exception of KPS. Interobserver concordance rates of KPS have been reported to range between $38 \%$ and $75 \%,{ }^{16,17}$ with the highest variability seen when dealing with patients with low performance scores. ${ }^{18}$ As all the DS-GPA scores have critical KPS cut-off points at 60 and 70, giving a lower or higher score around these values assigns patients a different DS-GPA score. However, in creating the DS-GPA scores, Sperduto et al. also used KPS as reported in a clinical setting, ${ }^{6-9}$ which underscores the predictive value of KPS despite its imperfect inter-observer-reliability. Furthermore, scoring models in which KPS categories are very broad (e.g. $\geq 70$ or $<70$ for melanoma) are less susceptible to inter-observer variability.

There are several limitations to this study. First of all, this is a retrospective cohort, meaning that all clinical information had to be extracted from patient files. Not all information had been recorded in the files, including tumour subtype and molecular markers. Although the DS-GPA could be calculated for all but I patient due to missing breast tumour receptor status, there was missing data in other tumour markers as well. For the NSCLC and melanoma groups, an "unknown" marker status option is included in the scoring model. This means that patients with missing marker status can still be assigned a GPA-score. However, this score does not reflect their true marker status, and thus misclassification can occur in these patients. This is especially likely in the NSCLC patients, as the majority of these patients had missing marker status. Aside for tumour markers, this potential misclassification also applies to the RCC patients and their Hb levels. 
Similarly, no DS-GPA could be calculated for lung cancer patients with missing pathological data, because information about tumour histology (NSCLC or SCLC) is needed to decide whether or not the lung-specific GPA is applicable. This is because the most recent lungspecific GPA scoring model, the lung-molGPA, is based solely on NSCLC patients. Please note that selecting SCLC is still an option on the brainmetgpa.com website, despite the fact that the subsequently used scoring model is not based on SCLC data.

Additionally, 24 NSCLC patients could not be included in the analysis of predicted and actual survival, due to missing information on tumour subtype (adenocarcinoma or nonadenocarcinoma).

As mentioned above, there were low numbers of patients in the DS-GPA groups, especially the lowest and highest ones, which reduces the power to show significant differences in median survival and in the Kaplan-Meier analysis.

Another limitation regards one of the applications of the DS-GPA score, which is to select the desired treatment based on expected survival. As completed SRS treatment was needed for inclusion in our cohort, we do not have data on all patients who were eligible for treatment, regardless of actually receiving it. We therefore cannot assess the performance of the DS-GPA in patients who did not receive SRS, which means we cannot address the aforementioned application of the DS-GPA. Similarly, we don't have enough data to reflect on the effect of newer targeted therapies on the performance of the DS-GPA model.

Lastly, in 30 patients (8.2\%), the interval between diagnosis and first treatment exceeded 2 months. These patients were excluded in Sperduto's patient cohorts used for creating the scoring models. We decided to include them, in order to reflect daily practice. In a posthoc test without these patients, no major differences were seen with the original analysis.

These limitations warrant further research within a larger prospective cohort, preferably from multiple centres. Should differences in survival persist, these pooled clinical data could be used for creating a SRS-specific prediction model, which best fits the patient population treated with radiosurgery. Recent observational data and an on-going randomized trial on the effect of SRS in patients with up to 15 brain metastases may aid in developing a model, in such a way as to reflect all patients eligible for SRS treatment. ${ }^{15,19}$

The way in which physicians and other health professionals discuss the results of the DS-GPA score is important. The fact that it results in a median survival and not a predicted survival is an important distinction. We have noticed that the result of the DS-GPA model is sometimes interpreted as a point prediction of survival, but this is beyond its scope. 
Patients should not be told that the DS-GPA gives a precise prediction of the expected survival. Instead, a patient needs to be informed that around half of the patients with similar clinical characteristics reach the median survival time, but that the other half dies before that time. We were able to replicate most of these median survival times, with some notable exceptions, as mentioned above. Additionally, the window of survival that applies to half of the patients, i.e. the interquartile range, is probably the most important message for patients, since it reflects the variation in survival more accurately than the median. We were also able to confirm the validity of the published interquartile ranges from the DS-GPA-models.

In our cohort of patients undergoing SRS for brain metastases of lung cancer, melanoma, renal cell carcinoma, breast cancer of gastrointestinal cancer, we were able to confirm the predictive value of the DS-GPA for median survival, with one notable exception. One subgroup, the intermediate-prognosis adeno-NSCLC patients, had a significantly shorter median survival then predicted by their DS-GPA score. Our findings confirm the value of the DS-GPA as a useful prognostic tool for the counselling of individual patients with brain metastases before undergoing SRS; while it does not provide a survival that is accurate for every patient, the predicted median and IQR could be reproduced on a group level. Additionally, it proved useful for accurately stratifying patients with both types of NSCLC, RCC and melanoma into prognostic subgroups. The limited number of patients within each subgroup and stratum of our cohort warrants replication in larger-scale prospective cohorts. 


\section{REFERENCES}

I. Nayak L, Lee EQ, Wen PY. Epidemiology of Brain Metastases. Current Oncology Reports. 20 I2; I4(I):4854. doi: |0.1007/s|1912-01|-0203-y

2. Schouten LJ, Rutten J, Huveneers HAM, Twijnstra A. Incidence of brain metastases in a cohort of patients with carcinoma of the breast, colon, kidney, and lung and melanoma. Cancer. 2002;94(I0):2698-2705.

3. Nussbaum ES, Djalilian HR, Cho KH, Hall WA. Brain metastases. Histology, multiplicity, surgery, and survival. Cancer. 1996;78(8):1781-1788.

4. Counsell CE, Collie DA, Grant R. Incidence of intracranial tumours in the Lothian region of Scotland, 1989-90. Journal of neurology, neurosurgery, and psychiatry. 1996;6I(2): 143-I50.

5. Lagerwaard FJ, Levendag PC, Nowak PJ, Eijkenboom WM, Hanssens PE, Schmitz PI. Identification of prognostic factors in patients with brain metastases: a review of 1292 patients. International journal of radiation oncology, biology, physics. 1999;43(4):795-803.

6. Sperduto PW, Kased N, Roberge D, et al. Summary Report on the Graded Prognostic Assessment: An Accurate and Facile Diagnosis-Specific Tool to Estimate Survival for Patients With Brain Metastases. Journal of Clinical Oncology. 2012;30(4):419-425. doi:I0.1200/JCO.201I.38.0527

7. Sperduto PW, Deegan BJ, Li J, et al. Estimating survival for renal cell carcinoma patients with brain metastases: an update of the Renal Graded Prognostic Assessment tool. Neuro-Oncology. Published online July 26, 2018. doi:10.1093/neuonc/noy099

8. Sperduto PW, Yang TJ, Beal K, et al. Estimating Survival in Patients With Lung Cancer and Brain Metastases: An Update of the Graded Prognostic Assessment for Lung Cancer Using Molecular Markers (Lung-moIGPA). JAMA oncology. 2017;3(6):827-831. doi:10.1001/jamaoncol.2016.3834

9. Sperduto PW, Jiang W, Brown PD, et al. Estimating Survival in Melanoma Patients With Brain Metastases: An Update of the Graded Prognostic Assessment for Melanoma Using Molecular Markers (Melanoma-molGPA). International Journal of Radiation Oncology*Biology*Physics. 2017;99(4):812-816. doi:10.1016/j.ijrobp.2017.06.2454

10. Sperduto PW. GPA Index. Published 2018. www.brainmetgpa.com

II. Landelijke richtlijn hersenmetastasen, versie 3.0. Landelijke werkgroep neuro-oncologie. Published 20Il. oncoline.nl

12. Yamamoto M, Serizawa T, Shuto T, et al. Stereotactic radiosurgery for patients with multiple brain metastases (JLGK090I): a multi-institutional prospective observational study. The Lancet Oncology. 2014;15(4):387-395. doi:I0.10।6/SI470-2045(14)7006I-0

13. Bhatnagar AK, Flickinger JC, Kondziolka D, Lunsford LD. Stereotactic radiosurgery for four or more intracranial metastases. International journal of radiation oncology, biology, physics. 2006;64(3):898-903. doi:I0.1016/j.jijrobp.2005.08.035

14. Garcia MA, Xu C, Nakamura JL, et al. Stereotactic Radiosurgery for $\geq 10$ Brain Metastases. International Journal of Radiation Oncology*Biology*Physics. 2017;99(2):E74-E75. doi:10.1016/j.jprobp.2017.06.769

15. Hughes RT, McTyre ER, LeCompte M, et al. Clinical Outcomes of Upfront Stereotactic Radiosurgery Alone for Patients With 5 to 15 Brain Metastases. Neurosurgery. Published online July 6, 2018. doi:10.1093/neuros/nyy276

16. Myers J, Gardiner K, Harris K, et al. Evaluating Correlation and Interrater Reliability for Four Performance Scales in the Palliative Care Setting. Journal of Pain and Symptom Management. 2010;39(2):250-258. doi:10.1016/j.jpainsymman.2009.06.013

17. Taylor AE, Olver IN, Sivanthan T, Chi M, Purnell C. Observer error in grading performance status in cancer patients. Supportive Care in Cancer. 1999;7(5):332-335. doi:10.1007/s00520005027I

18. Kelly CM, Shahrokni A. Moving beyond Karnofsky and ECOG Performance Status Assessments with New Technologies. Journal of Oncology. 2016;2016:I-13. doi:I0.1155/2016/6186543

19. Roberge D, Brown PD, Whitton A, et al. The Future Is Now-Prospective Study of Radiosurgery for More Than 4 Brain Metastases to Start in 2018! Frontiers in oncology. 2018;8:380. doi:10.3389/ fonc. 2018.00380 


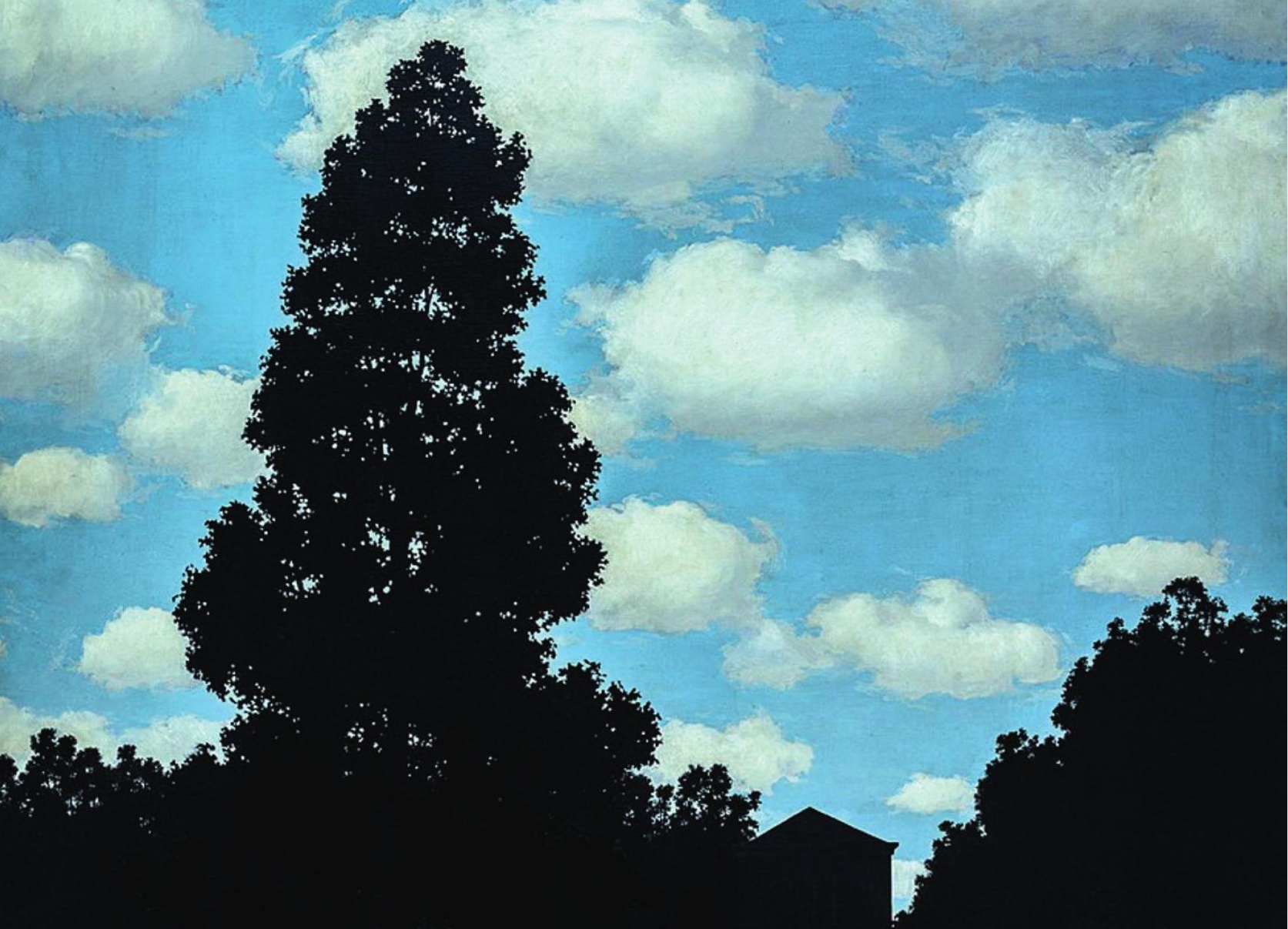

4t

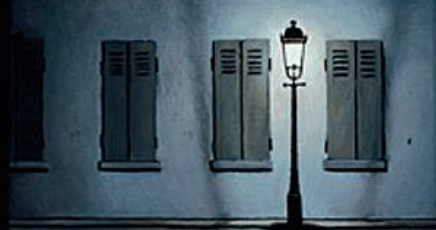




\section{Chapter 7}

\section{Age, pathology and CA-125 are prognostic factors for survival in patients with brain metastases from gynaecological tumours}

S.H.J. Nagtegaal, A.F.C. Hulsbergen, E.B.L. van Dorst, V.K. Kavouridis, C.A.C. Jessurun, M.L.D. Broekman, T. R. Smith, J.J.C. Verhoeff

Clinical and Translational Radiation Oncology 24 (2020): II-15 


\section{ABSTRACT}

\section{Background and purpose}

Brain metastases originating from gynaecological tumours are a rare phenomenon, but have an increasing incidence due to better targeted therapies. This study aimed to identify factors that predict survival in these patients, which can be used in creating a robust prognostic tool for shared decision making.

\section{Materials and methods}

We identified a consecutive cohort of 73 patients treated for gynaecological brain metastases in two tertiary institutions. Baseline demographics, pathology and serum CA- 125 were included in a multivariable Cox proportional hazards model.

\section{Results}

Median overall survival in our cohort was 14.4 months, with a one-year survival of $56.4 \%$ and a two-year survival of 39.1\%. Thirty-eight patients (52.1\%) had ovarian carcinoma as the primary malignancy. The following factors were significantly associated with survival: age (HR I.05 per year), CA-125 (HR 1.02 per $50 \mathrm{U} / \mathrm{ml}$ ), and uterine and vulvar primary tumours (when compared to ovarian carcinoma, with HRs 3.07 and 8.70). A post-hoc analysis with primary tumour site reclassified into ovary versus non-ovary showed a HR of 0.50 for ovarian primary tumour type.

\section{Conclusion}

We have found that age, pathology and CA-125 are prognostic factors for survival in patients with brain metastases from gynaecological tumours. Our findings may provide a foundation for future development of prediction models, for the benefit of both patients and physicians. 


\section{INTRODUCTION}

Brain metastases (BMs) occur in 20-40\% of all patients with cancer, with $8-10 \%$ having intracranial metastasis at time of diagnosis. ${ }^{1-5}$ The most frequent sites of origin being lung cancer, breast cancer and melanoma. Far less common primary tumours to metastasise to the brain are gynaecological tumours. The incidence of BMs from ovarian, endometrial and cervical carcinoma are estimated to be $0.3-2.2 \%,{ }^{6,7}$ Even rarer primary sites include vaginal and vulvar cancers, with only a handful of cases reported. 8,9 However, due to the advent of better targeted therapies, long-term survival in patients with gynaecological tumours has improved, with the unfortunate consequence of increased incidence of BMs. ${ }^{10}$ Additionally, advances in imaging have made early diagnosis of previously occult BMs possible, adding to the incidence. ${ }^{11,12}$

As is the case in all oncologic fields, survival prediction in BMs is important to inform shared decision making between physicians and patients. Several tools have been developed to predict survival for BM patients, the most predominant being the Recursive Partitioning Analysis (RPA) ${ }^{13}$ and the Diagnosis-Specific Graded Prognostic Assessment (DS-GPA). ${ }^{14}$ However, no prognostic models based on such large cohorts have been created specifically for BMs from gynaecological tumours. As a first step to such a model, we set out to identify prognostic factors for survival in a large, multi-institutional retrospective cohort of patients with BMs from gynaecological tumours.

\section{METHODS}

\section{Patient selection and data collection}

We retrospectively selected a consecutive cohort of patients who underwent radiation therapy (RT) and/or neurosurgical resection as initial treatment for BMs originating from gynaecological tumours between 2012 and 2017 in two tertiary academic medical centres in Boston, United States and Utrecht, The Netherlands.

As this study only involved retrospective chart review, the need to obtain informed consent was waived in both institutions. In the Boston hospital, Institutional Review Board approval was obtained to conduct the study. In the Dutch hospital, permission was obtained from the institutional ethics committee.

\section{Data collection and outcome}

Baseline data were collected from patient records. Collected data consisted of age at first treatment for BMs, number of BMs, presence of extracranial metastases, level of serum 
cancer antigen 125 (CA-125), Karnofsky performance status (KPS) at baseline, primary tumour type and interval between diagnosis of the primary tumour and the BMs.

The primary outcome was overall survival from the point of initial treatment for brain metastases.

\section{Statistical analysis}

Patterns of missing data were analysed, and when data was deemed to be missing at random, multiple imputation was performed. ${ }^{15,16}$

A Cox proportional hazards model was made with the following variables: age, number of BMs, presence of extracranial metastases, CA-125, KPS, primary tumour type and interval between diagnosis of the primary tumour and the BMs. In a post-hoc analysis, a Cox proportional hazards model was made comparing ovarian with non-ovarian primary tumours along with the other variables. Additionally, the dataset was split into an ovarian and non-ovarian group, and separate Cox regression models were fit for each group. Kaplan-Meier curves were used to visualize differences in survival when stratifying for CA-125 and primary tumour type. Finally, the number of brain metastases was dichotomized into I or $\geq 2$, as possible treatment options between these groups differ. ${ }^{17}$

Statistical analyses were performed with R 3.5.I (R Foundation for Statistical Computing, Vienna, Austria) using the 'mice' package.18

\section{RESULTS}

\section{Participants}

In total, 73 patients were treated for BMs of gynaecological tumours and were included in analysis. In 69 (94.5\%) of these patients, pathological examination of the brain lesions confirmed the diagnosis. There were missing data for CA-125 ( $n=16,21.9 \%)$ and KPS ( $n=1,1.4 \%$ ), with CA-125 only being determined for BMs of ovarian origin in one of the participating centres. Baseline characteristics after imputation are presented in Table I, and treatment characteristics are shown in Table 2. The entire cohort had a median survival of I4.4 months, with a one-year survival of $56.4 \%$ and a two-year survival of $39.1 \%$. 
Table I Baseline characteristics

\begin{tabular}{ll}
\hline & Total $\mathrm{n}=73$ \\
\hline Age (median; IQR) & $\mathbf{n}$ \\
Number of BMs (\%) & $63(55-69)$ \\
$\mid$ & \\
2 & $38(52.1)$ \\
3 & $15(20.5)$ \\
4 & $9(12.3)$ \\
$\geq 5$ & $4(5.5)$ \\
Extracranial metastases present (\%) & $7(4.1)$ \\
CAI25 (median; IQR) & $45(61.6)$ \\
KPS (median; IQR) & $38(9-97)$ \\
Primary tumour type (\%) & $80(80-100)$ \\
Ovary & \\
Cervix & $38(52.1)$ \\
Uterine sarcoma & $5(6.8)$ \\
Endometrium & $9(12.3)$ \\
Fallopian tube & $17(23.3)$ \\
Vulva & $2(2.7)$ \\
Interval primary diagnosis and BM in months & $2(2.7)$ \\
(median;IQR) & \\
\hline
\end{tabular}

$\mathrm{BM}=$ Brain metastasis; $\mathrm{IQR}=$ Inter quartile range; $\mathrm{KPS}=$ Karnofsky performance status; $\mathrm{SRS}=$ stereotactic radiosurgery; WBRT: whole brain radiotherapy

\section{Primary analysis}

Results of the Cox proportional hazards model are shown in Table 3. The following factors were significantly associated with survival: age (HR 1.05 per year), CA- 125 (HR I.02 per 50 $\mathrm{U} / \mathrm{ml}$ ), and uterine and vulvar primary tumours (with ovarian carcinoma as a reference, with HRs 3.07 and 8.70, respectively). A Kaplan-Meier plot comparing survival for the different primary tumour types is shown in Figure I, and a Kaplan-Meier plot comparing high and low CA- 125 values is shown in Figure 2. 
Table 2 Treatment characteristics

n

\section{Treatment for BMs (\%)}

Surgery

$9(12.3)$

RT

$7(9.6)$

$\mathrm{RT}+$ Surgery

$57(78.1)$

Type of RT (\%)

SRS

$36(49.3)$

SRT

$3(4.1)$

WBRT

$23(31.5)$

WBRT + SRS boost

Unknown

Dose of RT (\%)

$|x| 7$

$|x| 8$

$3(4.1)$

$1 \times 20$

I (I.4)

$|\times 2|$

$2(2.7)$

$1 \times 24$

$2(2.7)$

$3 \times 8$

$4(5.5)$

$5 \times 4$

$2(2.7)$

$5 \times 5$

$15(20.5)$

$5 \times 6$

$7(9.6)$

$10 \times 3$

$13(17.8)$

$13 \times 3$

I (I.4)

$14 \times 2.5$

$2(2.7)$

$15 \times 2.5$

$6(8.2)$

$17 \times 2$

I (1.4)

$20 \times 2$

I (1.4)

Unknown

$3(4.1)$

Treatments for multiple BMs (\%)

All BMs resected

$2(2.7)$

I BM resected; $\mathrm{RT}$ for other BMs

$16(21.9)$

I BM resected; no treatment for other BMs

$9(12.3)$

2 BMs resected; no treatment for other BMs

$4(5.5)$

$\mathrm{RT}$ for all BMs

Unknown

Interval surgery and RT in days (median; IQR) $28(22-38)$

$\mathrm{BM}=$ Brain metastasis; HFSRT = Hypofractionated stereotactic radiotherapy; RT = Radiotherapy; SRS = Stereotactic radiosurgery; WBRT $=$ Whole-brain radiotherapy 
Table 3 Results from the Cox proportional hazards model

\begin{tabular}{lll}
\hline Variable & HR (95\% Cl) & P \\
\hline Age & $1.05(1.01-1.09)$ & $\mathbf{0 . 0 3}$ \\
Number of BMs & $1.12(0.97-1.29)$ & 0.14 \\
Extracranial metastases present & $1.58(0.85-2.95)$ & 0.15 \\
CA-I25* & $1.02(1.01-1.03)$ & $<0.01$ \\
KPS & $1.00(0.98-1.02)$ & 0.74 \\
Primary tumour type & & \\
$\quad$ Ovary** & $-1.71(0.33-8.90)$ & - \\
Cervix & $3.07(1.12-8.42)$ & 0.52 \\
Uterine sarcoma & $1.93(0.95-3.94)$ & 0.03 \\
$\quad$ Endometrium & $0.51(0.06-4.06)$ & 0.07 \\
Fallopian tube & $8.70(1.19-63.34)$ & 0.52 \\
Vulva & $1.00(0.99-1.01)$ & $\mathbf{0 . 0 3}$ \\
Interval primary diagnosis and BM & 0.64 \\
\hline
\end{tabular}

$\mathrm{BM}=$ Brain metastases; KPS = Karnofsky Performance Status

*In steps of $50 \mathrm{U} / \mathrm{ml}$

**Reference

Overall survival by primary origin

Primary + Ovarian carcinoma + Cervical carcinoma + Uterine sarcoma + Endometrial carcinoma + Fallopian tube carcinoma

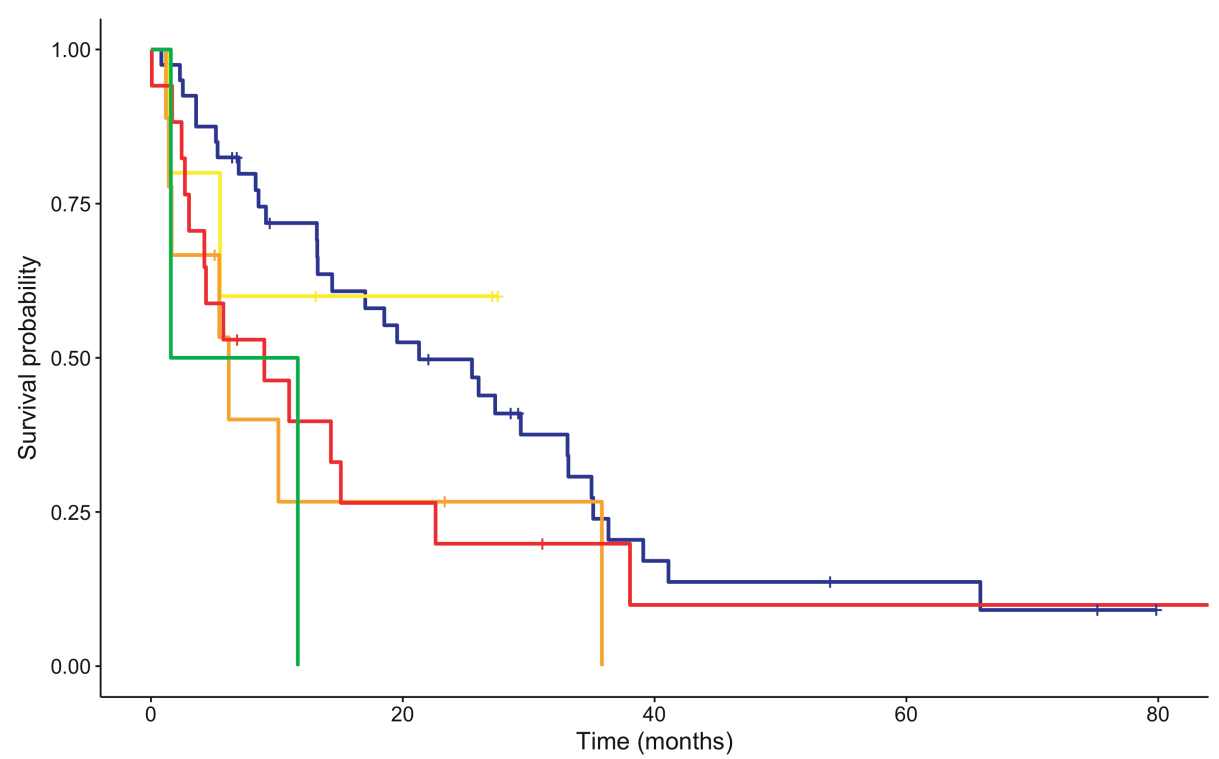

Figure I Kaplan-Meier plot comparing survival for the different primary tumour types 
Overall survival by CA-125

CA-125 + Elevated (> $35 \mathrm{U} / \mathrm{mL})+$ Normal

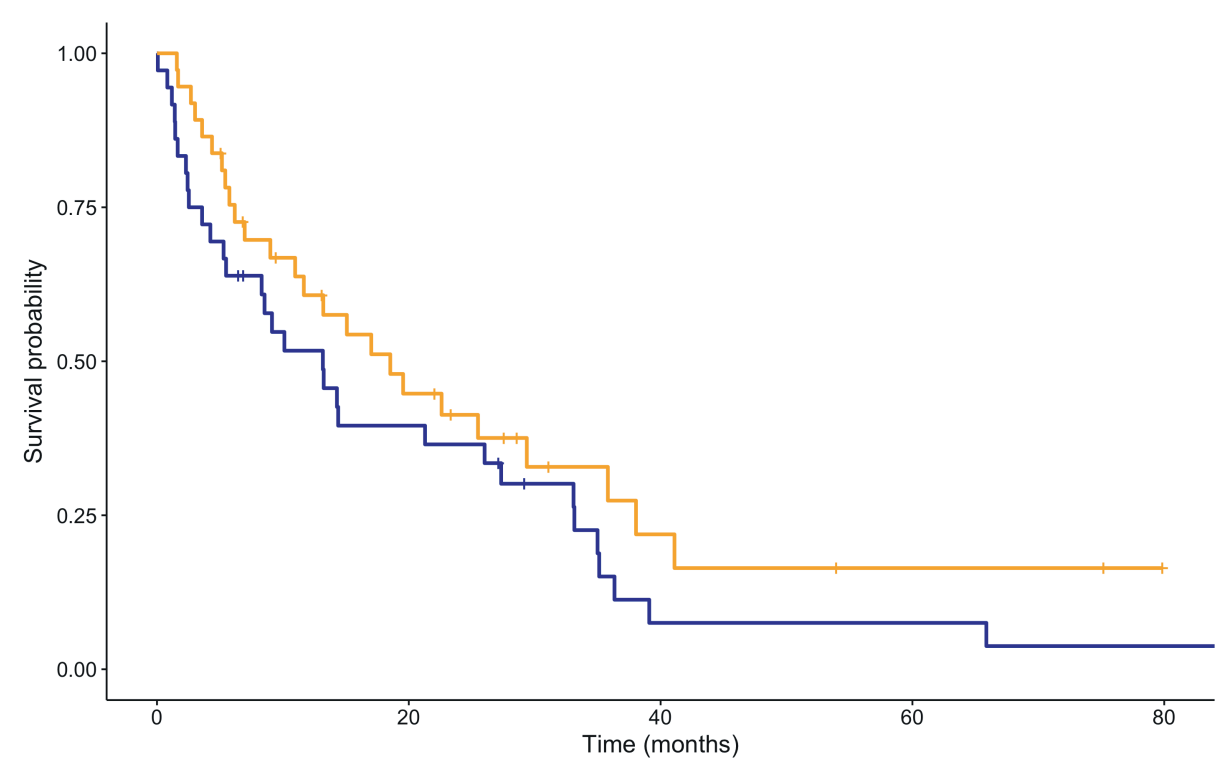

Figure 2 Kaplan-Meier plot comparing high and low serum CA-125 at baseline

\section{Secondary analyses}

A post-hoc Cox model with primary tumour site reclassified into ovarian vs. non-ovarian showed that ovarian origin $(\mathrm{HR} 0.50,95 \% \mathrm{Cl} 0.24-0.93, \mathrm{p}=0.03)$ and fewer $\mathrm{BMs}(H \mathrm{R}$ I.I5, $95 \% \mathrm{Cl}$ ।.20-1.31, $p=0.03$ ) were associated with improved survival. A Kaplan-Meier plot of survival comparing ovarian vs. non-ovarian primary tumour is shown in Figure 3.

Additionally, the number of brain metastases was dichotomized into 1 or $\geq 2$, which resulted in a significant result $(H R$ I.9, 95\% Cl $1.003-3.52, p=0.049)$.

In order to assess the effect of missing data and subsequent imputation of CA-125, a sensitivity analysis was performed exclusively incorporating cases with a known CA-125 value $(n=57)$. This showed no changes from principal analysis, indicating that the effect of missing data was negligible. Another sensitivity analysis was performed by splitting the dataset into ovarian and non-ovarian groups. CA-125 was only significant in the ovarian subgroup $(p<0.01)$, with no prognostic effect seen in the non-ovarian group. 


\section{Overall survival by primary origin}

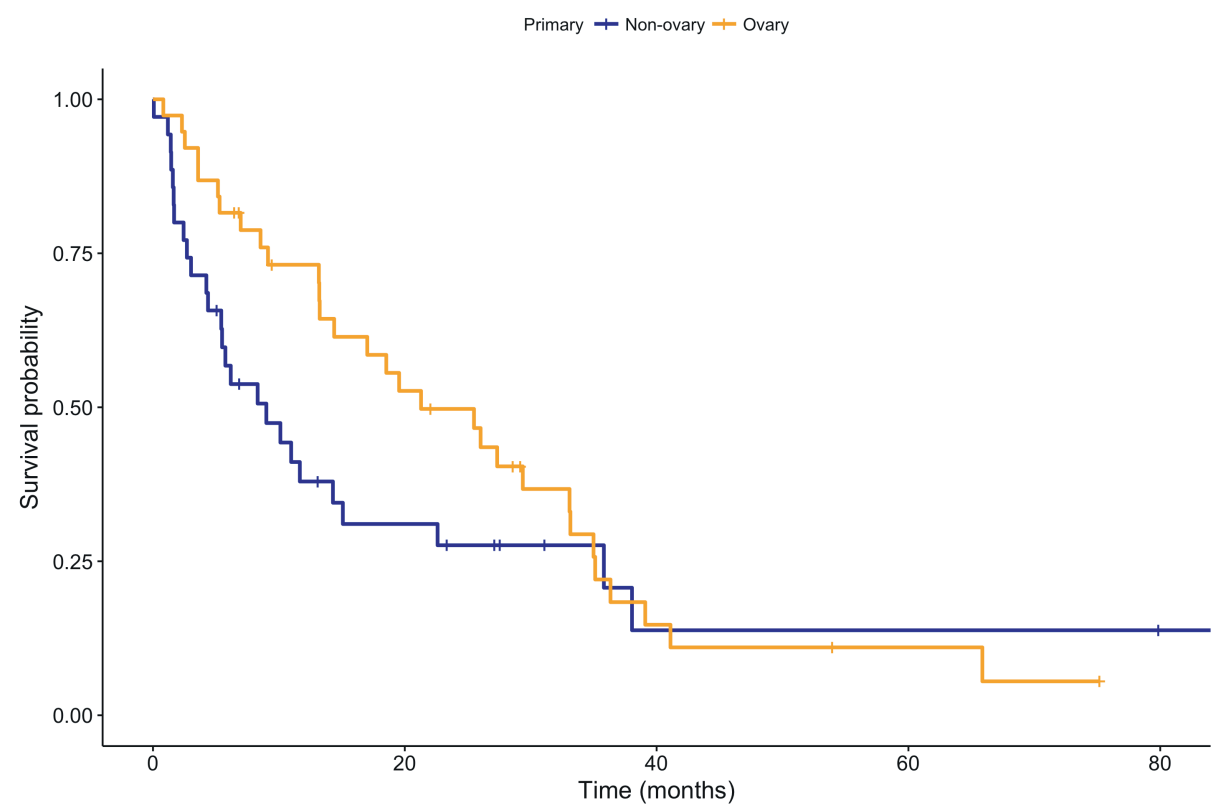

Figure 3 Kaplan-Meier plot comparing ovarian vs non-ovarian primary tumour (adjusted HR 0.50, $\mathrm{Cl} 0.27-0.93, p=0.03)$

\section{DISCUSSION}

In this study, we have identified several independent factors that are prognostic for survival in brain metastases patients with gynaecological tumours. In particular, primary tumour origin seems to be a strong predictor, along with CA-125. Additionally, in post-hoc analysis, a significant effect was found when comparing I and $\geq 2 \mathrm{BMs}$, suggesting that not the absolute number, but the solitary or multiple nature of BMs has an effect on survival.

Several other studies have attempted to identify prognostic factors in gynaecological brain metastases.

In the biggest cohort, from the MITO 19 study, 174 women with BMs from epithelial ovarian cancer were included..$^{19}$ After multivariable analysis, the following variables were significantly associated with survival: multiple BMs, extracranial metastases, age, and monotherapy.

In another cohort, Matsunaga et al. ${ }^{20}$ presented an analysis of 70 patients undergoing Gamma Knife Surgery (GKS) for BMs from gynaecological tumours. They found significant prognostic effects for type of primary lesion, controlled extracranial disease (compared 
to active) and number of BMs ( $\mathrm{V}$ vs. $\geq 2$ ). Similar to our results, KPS was not found to be significant, with and $95 \% \mathrm{Cl}$ of the HR 0.38-1.36. In a similar, smaller cohort of 33 patients undergoing GKS, Johnston et al. found age and RPA to significantly predict survival.. ${ }^{21}$

Rades et al. ( $n=56$ ) examined the prognostic value of several clinical factors in patients treated with surgery and/or radiotherapy. ${ }^{8}$ A multivariable model showed significant prognostic value of Eastern Cooperative Oncology Group (ECOG) performance score, as well as the absence of extracranial metastases. Janssen et al. $(n=49)$ found a similar effect for ECOG score in a subgroup of patients who only received WBRT, but found no significant effect of extracranial metastasis. ${ }^{9}$ Finally, Anupol et al. studied 15 patients, and found that type of therapy and the presence of extracranial metastases predict survival. ${ }^{22}$

These results have been further used to create two nomograms by Janssen et al. and Rades et al. to predict survival.8,9 However, these were based on cohorts too small to make robust prediction models, and have not been validated in separate cohorts. Therefore, they have seen sparse use in clinical practise.

Serum CA- 125 has been a factor of interest before in patients with gynaecological cancers who developed brain metastases. In the abovementioned study performed by Anupol et al. ten out of fifteen BM patients were found to have an elevated CA-125, although the authors found no correlation with survival. ${ }^{22}$ Similar results have been found in other small cohorts (with sample sizes ranging from 4 to 15, see also the review by Piura et al. ${ }^{23}$ ), leading to the conclusion that serum CA-125 is not an effective biomarker for gynaecological BMs, nor a useful predictor for survival. However, the small sample sizes of these studies have limited statistical power, potentially yielding false-negative results. Our study, which is the first to investigate this variable in a large sample size, challenges these findings from previous literature.

In gynaecological practice, CA-125 is used in the diagnostic workup for ovarian cancer. On its own, the result of CA-125 testing is less reliable than other diagnostic tests like ultrasonography. ${ }^{24}$ It has also been considered as a marker for cancer screening in the general population, but a recent review found no evidence for a beneficial effect on survival. ${ }^{25} \mathrm{~A}$ likely reason for the limited performance of CA- 125 as a diagnostic tool on its own is the fact that its level can be influenced by non-cancer related factors, like obesity, age, phase of the menstrual cycle, menopause, smoking status and history of hormone therapy. ${ }^{26,27}$ Aside from these issues with specificity, the sensitivity is also limited, as only $50 \%$ of patients with early stage ovarian cancer have elevated CA-125 levels. Therefore, the interpretation of CA-125 level should not be done without considering different clinical factors and imaging. ${ }^{28}$ As a prognostic factor, however, CA- 125 has shown more reliability, 
as it has been demonstrated to strongly predict both overall and progression free survival in patients with ovarian cancer. ${ }^{29}$ Additionally, it has been shown to detect cancer recurrence long before symptoms occur. ${ }^{30}$ However, the added value of repetitive post-treatment CA-125 surveillance is limited, as direct treatment at relapse detected solely by this marker (i.e. with no signs or symptoms present) does not result in a survival advantage. ${ }^{31}$ Therefore, the clinical decision-making should not be affected by CA-125 alone, and it should be considered only as one of several prognostic factors for patients' survival.

The observed difference in survival between ovarian and non-ovarian primary could be explained due to differing survival per tumour type, regardless of the presence of BMs. Ovarian cancer has a more favourable survival compared to other gynaecological cancers. ${ }^{32}$ Different options with regards to targeted therapies and diagnostic methods may also influence survival.

The fact that KPS does not predict survival in our study is at odds with previous studies, which found prognostic effects of the ECOG score. ${ }^{8,9}$ The reason for this is not clear. It could be the case that differences in variables in the respective prognostic models causes the effect of performance status to differ between studies, as different clinical factors are corrected for. Also, the choice of performance status (KPS or ECOG) can be the cause of this discordance, as Matsunaga et al. and Johnston et al. also examined the effect of KPS, and found no significant effect. ${ }^{20,21} \mathrm{~A}$ final possibility is that, due to the relatively high KPS in our dataset (mean 80, with an IQR of 80-100), the effects of lower KPS are harder to determine.

As with most retrospective analyses, the biggest limitation of the current study is missing data. Even though we have selected a validated way of imputing our dataset ${ }^{15,16}$, we cannot be sure our results would have been the same if no data were missing. However, a sensitivity analysis with only observed values shows similar results to our primary analysis, suggesting that the effect of missing data is negligible.

Similarly, other variables that could be of interest were not recorded, and could therefore not be analysed. It has been suggested that gross tumour volume could be a more predictive factor for survival than number of BMs. ${ }^{33}$ We would have liked to explore this further, but were limited by the unfeasibility to collect these data.

Additionally, local differences in clinical protocol meant that in one centre CA-125 was only measured in patients with BMs of ovarian origin. A sensitivity analysis was done by splitting the dataset into ovarian and non-ovarian groups, which resulted in a significant prognostic effect of CA-125 in the ovarian group only. However, the sample size in the non-ovarian group was limited, suggesting there might have been insufficient power to find a significant 
result. Additionally, the sensitivity analysis with only observed values showed a significant predictive effect of CA-125 independent from primary tumour type.

Finally, our limited sample size limits our ability to detect smaller prognostic effects. This is an unfortunate inherency in research into rare diseases. The lack of a large number of participants also hinders our ability to create a robust and widely applicable nomogram to predict survival.

This latter point is our main suggestion for the future. Our hypothesis-generating results can be used to inform the creation of a nomogram for gynaecological BMs. This should be based on a large cohort of patients, ideally from multiple centres with different treatment patient demographics. This prediction model will aid the decision-making process and helps guide the patient and physician to the most optimal treatment, in order to provide the best possible care.

In conclusion, we have found that age, pathology and CA-125 may be prognostic factors for survival in brain metastasis from gynaecological tumours. The predictive role of CA-125 in BMs from non-ovarian origin is less clear, and remains to be further investigated. Our findings may help to inform clinical decision making, as well as identify variables of interest for the construction of robust nomograms from large, multi-institutional databases. 


\section{REFERENCES}

I. Andrews DW, Scott CB, Sperduto PW, et al. Whole brain radiation therapy with or without stereotactic radiosurgery boost for patients with one to three brain metastases: phase III results of the RTOG 9508 randomised trial. Lancet (London, England). 2004;363(9422):1665-1672. doi:10.1016/ S0140-6736(04)16250-8

2. Hasegawa T, Kondziolka D, Flickinger JC, Germanwala A, Lunsford LD. Brain Metastases Treated with Radiosurgery Alone: An Alternative to Whole Brain Radiotherapy? Neurosurgery. 2003;52(6):13181326. doi:I0.1227/0I.NEU.0000064569.189|4.DE

3. Nayak L, Lee EQ, Wen PY. Epidemiology of Brain Metastases. Current Oncology Reports. 2012;14(I):4854. doi:10.1007/s11912-011-0203-y

4. Berghoff AS, Schur S, Füreder LM, et al. Descriptive statistical analysis of a real life cohort of 2419 patients with brain metastases of solid cancers. ESMO open. 2016;1(2):e000024. doi:10.1136/ esmoopen-2015-000024

5. Cagney DN, Martin AM, Catalano PJ, et al. Incidence and prognosis of patients with brain metastases at diagnosis of systemic malignancy: a population-based study. Neuro-Oncology. 20 |7;|19(| I): I5 I I-I521. doi:10.1093/neuonc/nox077

6. Nasu K, Satoh T, Nishio S, et al. Clinicopathologic features of brain metastases from gynecologic malignancies: A retrospective study of 139 cases (KCOG-GI00Is trial). Gynecologic Oncology. 2013;128(2):198-203. doi:10.1016/j.ygyno.2012.11.001

7. Hacker NF, Rao A. Surgical management of lung, liver and brain metastases from gynecological cancers: a literature review. Gynecologic Oncology Research and Practice. 2016;3(1):7. doi:10.1186/ s4066I-016-0028-3

8. Rades D, Janssen S, Bajrovic A, Veninga T, Fischer D, Schild SE. A New Scoring Tool to Assess Overall Survival in Patients With Intracerebral Metastases From Gynecological Cancers. International journal of gynecological cancer : official journal of the International Gynecological Cancer Society. 20 17;27(3):597602. doi:I0.1097/IGC.0000000000000899

9. Janssen S, Hansen HC, Schild SE, Rades D. An Instrument for Estimating the 6-Month Survival Probability After Whole-brain Irradiation Alone for Cerebral Metastases from Gynecological Cancer. Anticancer research. 2018;38(6):3753-3756. doi:10.21873/anticanres. 12656

10. Gilani MA, Williams NL, Giordano C, et al. Brain Metastases in Patients with Gynecologic Cancers: A Single Institution Experience and Review of the Literature. Open Journal of Obstetrics and Gynecology. 2016;06(09):544-552. doi:10.4236/ojog.2016.69070

II. Seute T, Leffers P, ten Velde GPM, Twijnstra A. Detection of brain metastases from small cell lung cancer: consequences of changing imaging techniques (CT versus MRI). Cancer. 2008; I I2(8):I827-I834. doi:|0.1002/cncr.2336|

12. Pope WB. Brain metastases: neuroimaging. Handbook of clinical neurology. 20 I8; |49:89- II2. doi: I0.1016/ B978-0-12-811161-1.00007-4

13. Gaspar L, Scott C, Rotman M, et al. Recursive partitioning analysis (RPA) of prognostic factors in three radiation therapy oncology group (RTOG) brain metastases trials. International Journal of Radiation Oncology*Biology*Physics. 1997;37(4):745-751. doi:10.1016/S0360-3016(96)00619-0

14. Sperduto PW, Kased N, Roberge D, et al. Summary Report on the Graded Prognostic Assessment: An Accurate and Facile Diagnosis-Specific Tool to Estimate Survival for Patients With Brain Metastases. Journal of Clinical Oncology. 2012;30(4):419-425. doi:I0.1200/JCO.2011.38.0527

15. Sterne JAC, White IR, Carlin JB, et al. Multiple imputation for missing data in epidemiological and clinical research: potential and pitfalls. BMJ. 2009;338(jun29 I):b2393-b2393. doi:I0.1 I36/bmj.b2393

16. Moons KGM, Donders RART, Stijnen T, Harrell FE. Using the outcome for imputation of missing predictor values was preferred. Journal of clinical epidemiology. 2006;59(I0):1092-1 I01. doi:10.1016/j. jclinepi.2006.01.009

17. Landelijke richtlijn hersenmetastasen, versie 3.0. Landelijke werkgroep neuro-oncologie. Published 20II. oncoline.nl

18. R Core Team. R: A language and environment for statistical computing. R Foundation for Statistical Computing. Published online 2017. 
19. Marchetti C, Ferrandina G, Cormio G, et al. Brain metastases in patients with EOC: Clinicopathological and prognostic factors. A multicentric retrospective analysis from the MITO group (MITO 19). Gynecologic oncology. 2016;143(3):532-538. doi:I0.1016/j.ygyno.2016.09.025

20. Matsunaga S, Shuto T, Sato M. Gamma Knife Surgery for Metastatic Brain Tumors from Gynecologic Cancer. World neurosurgery. 2016;89:455-463. doi:10.1016/j.wneu.2016.01.062

21. Johnston H, McTyre ER, Cramer CK, et al. Stereotactic radiosurgery in the treatment of brain metastases from gynecologic primary cancer. Journal of radiosurgery and SBRT. 2017;5(I):55-61.

22. Anupol N, Ghamande S, Odunsi K, Driscoll D, Lele S. Evaluation of Prognostic Factors and Treatment Modalities in Ovarian Cancer Patients with Brain Metastases. Gynecologic Oncology. 2002;85(3):487492. doi:I0.1006/gyno.2002.6653

23. Piura E, Piura B. Brain Metastases from Ovarian Carcinoma. ISRN Oncology. 2011;2011:1-13. doi: $10.5402 / 2011 / 527453$

24. Dodge JE, Covens AL, Lacchetti C, et al. Preoperative identification of a suspicious adnexal mass: a systematic review and meta-analysis. Gynecologic oncology. 2012;126(1):157-166. doi:10.1016/j. ygyno.2012.03.048

25. Henderson JT, Webber EM, Sawaya GF. Screening for Ovarian Cancer. JAMA. 2018;319(6):595. doi:10.1001/jama.2017.21421

26. Johnson CC, Kessel B, Riley TL, et al. The epidemiology of CA-125 in women without evidence of ovarian cancer in the Prostate, Lung, Colorectal and Ovarian Cancer (PLCO) Screening Trial. Gynecologic oncology. 2008; II0(3):383-389. doi:I0.10 I6/j.ygyno.2008.05.006

27. Masahashi T, Matsuzawa K, Ohsawa M, Narita O, Asai T, Ishihara M. Serum CA 125 levels in patients with endometriosis: changes in CA 125 levels during menstruation. Obstetrics and gynecology. 1988;72(3 Pt I):328-331.

28. Bottoni P, Scatena R. The Role of CA 125 as Tumor Marker: Biochemical and Clinical Aspects. In: 2015:229-244. doi:10.1007/978-94-017-7215-0_14

29. Gupta D, Lis CG. Role of CAI25 in predicting ovarian cancer survival - a review of the epidemiological literature. Journal of ovarian research. 2009;2:13. doi:10.1186/1757-2215-2-13

30. Clarke T, Galaal K, Bryant A, Naik R. Evaluation of follow-up strategies for patients with epithelial ovarian cancer following completion of primary treatment. The Cochrane database of systematic reviews. 2014;(9):CD006|19. doi:10.1002/14651858.CD006119.pub3

31. Rustin GJ, van der Burg M EL, Griffin CL, et al. Early versus delayed treatment of relapsed ovarian cancer (MRC OV05/EORTC 55955): a randomised trial. The Lancet. 2010;376(9747):1।55-1I63. doi:10.1016/S0I40-6736(I0)61268-8

32. Netherlands Comprehensive Cancer Organization. Netherlands Cancer Registry. Published 2019. www.cijfersoverkanker.nl

33. Yamamoto M, Serizawa T, Shuto T, et al. Stereotactic radiosurgery for patients with multiple brain metastases (JLGK090I): a multi-institutional prospective observational study. The Lancet Oncology. 2014;15(4):387-395. doi:10.1016/SI470-2045(14)70061-0 
Prognostic factors for brain metastases from gynaecological tumours 


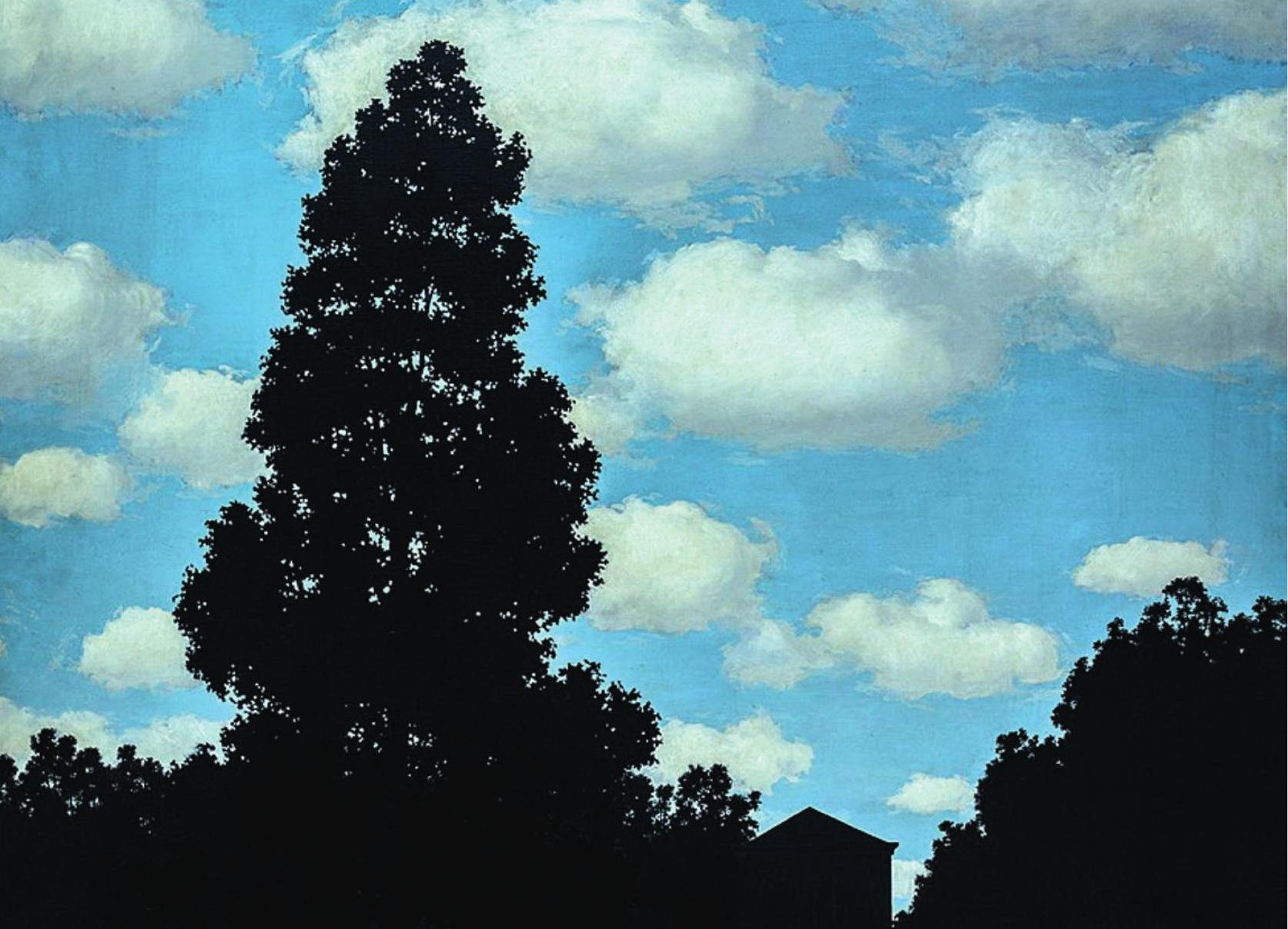

4t

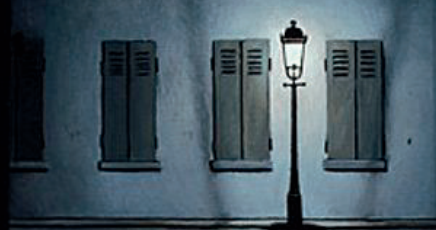




\section{Chapter 8}

Socioeconomic status is not associated with survival in patients with brain metastases treated with stereotactic radiotherapy

S.H.J. Nagtegaal, S.G. Elias, T.J. Snijders, H.M. Verkooijen, J.J.C. Verhoeff 


\section{ABSTRACT}

\section{Background and purpose}

In most cancer sites a low socioeconomic status (SES) is consistently associated with poorer survival. For brain metastasis, this relation is not well understood. Therefore, we studied the effect of SES on survival in Dutch brain metastasis patients treated with stereotactic radiosurgery in a tertiary radiotherapy facility.

\section{Materials and methods}

We retrospectively studied 404 consecutive patients treated with stereotactic radiosurgery for brain metastases in a tertiary referral centre between 2012 and 2017. Baseline prognostic factors for survival were collected. The SES score was based on education, income and employment. Cox proportional hazard models were made, corrected for the relevant variables identified from a directed acyclic graph (DAG). Adjustments were made in two ways in order to obtain comprehensive results: correcting for confounders (total effect), and correcting for confounders and mediating factors (direct effect).

\section{Results}

Unadjusted, estimation of the linear effect of SES on survival resulted in a HR of $0.92(95 \% \mathrm{Cl}$ 0.82 - 1.04). The total effect of SES was achieved by correcting for age, resulting in a HR of 0.95 , with a $95 \% \mathrm{Cl}$ of $0.84-1.07$. The direct effect gave a $\mathrm{HR}$ of $0.96(95 \% \mathrm{Cl} 0.84-1.10)$.

\section{Conclusion}

SES was not associated with survival in patients with brain metastases undergoing radiation therapy. When correcting for clinical variables, we found no significant relationship between SES and survival, with HRs suggesting limited clinical impact. The results suggest that patients' survival outcomes after contemporary cancer treatment are unrelated to their employment status, education status and annual income. 


\section{INTRODUCTION}

In patients with brain metastases median survival after diagnosis ranges from 2 to 12 months, depending on the primary tumour site., ${ }^{1,2}$ Brain metastases occur in 20-40\% of all cancer patients, with $8-10 \%$ having intracranial metastasis at initial presentation. ${ }^{1-5}$ The occurrence of brain metastases has a negative impact on survival of cancer patients, as well as affecting quality of life negatively. Treatment options for brain metastases are surgery, systemic treatment, and radiotherapy (RT), of which there are two main types: whole-brain radiotherapy and stereotactic radiotherapy (SRT). The latter can be delivered fractionated (FSRT) or in a single dose, referred to as stereotactic radiosurgery (SRS). New therapies are constantly being developed in order to improve survival after treatment. Besides therapy, other factors can have an effect on a patients' life expectancy as well.

One of these is the socioeconomic status (SES). Several reviews have shown that in most major cancer sites a low SES is consistently associated with poorer survival compared with a high SES. ${ }^{6-9}$ These studies and reviews analysed patients from several countries in North America, Europe and Asia, with varying healthcare systems (single and multi-payer, single and multiple provider).

Factors that contribute to a patient's SES, such as education level, income, and health insurance coverage, each influence access to healthcare, which in turn causes disparities in the rate of early detection, preventative medicine, adequate treatment and palliative care. ${ }^{9}$ Additionally, lifestyle factors like healthy diet, smoking, alcohol consumption and regularity of exercise are correlated with SES. As with many sociological phenomena, the general effect of SES on health is complexly interlinked with other cultural factors, including attitudes towards health, belief in modern medicine, and racial bias. ${ }^{10,11}$

Only a handful of papers have examined SES as a prognostic factor for survival in patients with brain metastases, with varying results. Most find no significant relation between SES and survival, 12,13 while one Dutch and one Chinese study did see a significant effect.14,15

The Dutch study, recently conducted by Ten Berge et al., studied a cohort of I 29 Dutch patients with synchronously diagnosed brain metastases and non-small-cell lung carcinoma (NSCLC) treated with Gamma Knife radiosurgery, and analysed whether SES influenced referral patterns and survival. Compared with low SES, patients with intermediate and high SES had a more favourable survival, with HRs of 0.9 and 0.8 .

We conducted a similar analysis to Ten Berge et al. in a cohort more representative of the brain metastasis population. We selected patients referred to a tertiary radiotherapy facility 
in the Netherlands for linac-based radiosurgery of both synchronous and non-synchronous brain metastases from multiple primary tumour sites.

\section{METHODS}

\section{Participants and data collection}

We identified all consecutive patients who were referred to the radiation oncology department of the UMC Utrecht for first SRS or FSRT treatment of newly diagnosed brain metastases (both synchronous and non-synchronous) between 1-1-2012 and 31-12-2017. Patients were eligible for inclusion if they had a primary tumour included in the diseasespecific Graded Prognostic Assessment (DS-GPA) model, i.e. NSCLC, breast cancer, melanoma, renal cell cancer (RCC), and gastro-intestinal (Gl) tumours.

We collected information on the following prognostic factors of the DS-GPA at baseline from electronic patient records: Karnofsky performance status (KPS), age, number of brain metastases, presence of extra-cranial metastases and disease-specific tumour markers. The following markers were collected: BRAF gene status (for melanoma), EGFR/ ALK gene status (for NSCLC), and HER2, and ER/PR receptor status (for breast cancer). Additionally, $\mathrm{Hb}$ (converted from $\mathrm{mmol} / \mathrm{l}$ to $\mathrm{g} / \mathrm{dL}$ ) was collected for RCC from standardized laboratory examination of blood samples.

The determinant SES was determined at baseline. The Netherlands Institute for Social Research (In Dutch: Sociaal en Cultureel Planbureau, SCP) periodically collects data on SES per postal code ${ }^{16}$. For each postal code, they summarize the SES in a score, based on education level, income and employment status. The score is based on a principal component analysis and is standardised, with a national average over time of 0 , and a standard deviation of I. This means each postal code receives a single score which has no intrinsic value, but is designed to allow comparisons with other regions over time.

The primary outcome of the study was overall survival in months after the start of RT. Data on survival was retrieved from patient records and the Municipal Personal Records Database (Gemeentelijke Basisadministratie, GBA). Patients who were still alive were censored on the date the GBA was consulted.

The need for informed consent was waived. We obtained permission from the Ethics Board of our institution to conduct this study with reference number 18/273. To ensure protection of private data, postal codes were retrieved by a specialised data manager. 


\section{Imputation}

To account for missing data we used multiple imputation. All clinical variables, including outcome, were used to impute missing values. ${ }^{17}$ In order to improve imputation results, the Nelson-Aalen estimator for the cumulative hazard was also included as an approximation of the baseline cumulative hazard ${ }^{18}$. The imputation process had 20 iterations and 10 imputations.

\section{Statistical analysis}

We determined the median follow-up time using the reverse Kaplan-Meier method. ${ }^{19}$ To identify the factors that need to be corrected for, a directed acyclic graph (DAG) was made with all collected clinical variables, using DAGgity v3.0. ${ }^{20}$ The relation between the factors in the DAG was determined with clinical expertise and opinion from an experienced clinician. The DAG allowed us to examine two effects of SES on survival, the total and direct effects. The reason for this distinction is that a determinant can have an effect on the outcome in multiple ways: directly, and through mediating factors. For example, someone's age might influence the odds of developing cancer. However, age can also have an impact on lifestyle factors (such as smoking or alcohol consumption) which in turn also affects cancer incidence. In other words, lifestyle would be a mediating factor. In this example, the effect of age on cancer incidence directly is the direct effect, and the effect through lifestyle is the indirect effect. Combining the direct and indirect effects gives the total effect, i.e. all possible ways the determinant affects the outcome. Please note that for both direct and total effects, confounding has to be taken into account. With the DAG, you can discover which variables you should correct for to achieve the desired effect: all confounders for the total effect, and all confounders and mediating factors for the direct effect. By looking at and comparing the total and direct effects, it can be concluded whether there was a relation between SES and survival, and whether this association is mostly explained through mediating factors, or only through the direct effect.

We fitted Cox proportional hazards models with SES as a continuous variable, corrected for the variables identified by the DAG to obtain the total and direct effects, and survival time as the outcome. The proportional hazards assumption was assessed for each model by correlating the scaled Schoenfeld residuals with time, confirming their independence. Variables that led to violation of this assumption were entered to the model as strata. Linearity of both SES and mediating factors was assessed by including them as a 5 -knot spline, and checking whether this improved model fit with likelihood ratio tests and the Akaike information criterion (AIC).

The SES score was divided into tertiles (in line the instructions provided by the SCP), and the survival in each group was estimated with Kaplan Meier analysis to obtain 
median survival, I-year and 2-year survival rates, and a survival plot. Comparison of the survival difference between the SES tertiles was done with a log-rank test, as well as Cox proportional hazards models corrected to obtain the total and direct effects. To account for possible confounding, an adjusted Kaplan Meier analysis was also estimated balancing the SES tertiles for differences in clinical variables by using inverse probability weighting (IPW). Weights were determined with multinomial regression, reflecting the probability of a subject having their observed SES tertile, given all potential predictive variables. ${ }^{21,22}$ We performed this analysis twice, with the variables needed for both the total and the direct effect, in each using age with a 5-knot spline. From each analysis, we obtained a survival plot, median survival, and I-year and 2-year survival rates.

Both the Cox proportional hazards modelling and Kaplan Meier analysis were done within the individual imputed datasets, and then pooled. For the HR's, pooling was done in accordance with the Rubin's rules. ${ }^{23}$ For IPW adjustment, the weights from the datasets were averaged for each patient.

We calculated effect estimates with corresponding 95\% confidence intervals, and considered a two-sided p-value smaller than 0.05 as statistically significant. Statistical analyses were performed with R 3.5.I (R Foundation for Statistical Computing, Vienna, Austria) with use of the packages 'survival' v3.I-8, 'mice' v3.8.0, 'ipw' vI.0-II, and 'IPWsurvival' v0.5. ${ }^{24}$

\section{RESULTS}

\section{Participants}

Between 2012 and 2017, 466 patients were treated with SRS or FSRT for brain metastases. Of those, 404 had one of the five primary tumour types of interest. Median follow-up time was 42.5 months (IQR 29.3 - 67.6), during which 348 (86.1\%) patients deceased. The SES ranged from -2.7 to 2.6 , with a median of 0.5 , and an IQR of $0.1-1.0$. There were relatively more men in the highest SES tertiles, and NSCLC was more present in the lowest tertile (Table I). Other characteristics were well distributed among the SES groups. Single-fraction SRS was used in 233 patients (57.7\%), with the rest receiving FSRT (Table 2). Multiple imputation was performed and checked, resulting in a complete dataset of 404 cases. Thirty-eight patients (9.4\%) had missing data, of the following variables: NSCLC adenocarcinoma yes or no (25 missings, 6.2\%), BRAF mutation (I; 0.2\%), breast cancer pathology (2; 0.5\%), Hb (9; 2.2\%), and SES score (I; 0.2\%).

\section{Effect of SES on survival}

The entire cohort had a median survival of 10.1 months (95\% Cl 8.4-11.9), with a I-year and 2 -year survival of $44.6 \%(95 \% \mathrm{Cl} 40.0-49.7) 25.8 \%(95 \% \mathrm{Cl} 21.8-30.4)$. 
Unadjusted, estimation of the effect SES on survival resulted in a $\mathrm{HR}$ of $0.92(95 \% \mathrm{Cl} 0.82$ - 1.04) for each unit increase in SES score, representing I standard deviation within the normal population. The DAG which was used to identify variables for which to correct is shown in Figure I. All collected clinical variables were used in creating the DAG.

Table I: Baseline characteristics of included patients

\begin{tabular}{|c|c|c|c|c|}
\hline & & $\begin{array}{c}\text { Low SES } \\
\begin{array}{c}{[-2.7-0.2]} \\
n=136\end{array}\end{array}$ & $\begin{array}{c}\text { Mid SES } \\
{[0.2-0.8]} \\
n=133\end{array}$ & $\begin{array}{c}\text { High SES } \\
{[0.8-2.6]} \\
n=134\end{array}$ \\
\hline $\begin{array}{l}\text { Age at diagnosis in } \\
\text { years, mean (SD) }\end{array}$ & & $62(10)$ & $62(10)$ & $64(10)$ \\
\hline Sex, n (\%) & $\begin{array}{l}\text { Male } \\
\text { Female }\end{array}$ & $\begin{array}{l}59(43.4) \\
77(56.6)\end{array}$ & $\begin{array}{l}63(47.4) \\
70(52.6)\end{array}$ & $\begin{array}{l}76(56.7) \\
58(43.3)\end{array}$ \\
\hline KPS, n (\%) & $\begin{array}{l}\leq 70 \\
80 \\
90-100\end{array}$ & $\begin{array}{l}49(36.0) \\
54(39.7) \\
33(24.3)\end{array}$ & $\begin{array}{l}38(28.6) \\
54(40.6) \\
41(30.8)\end{array}$ & $\begin{array}{l}46(34.3) \\
46(34.3) \\
42(31.3)\end{array}$ \\
\hline Tumour type, n (\%) & $\begin{array}{l}\text { NSCLC } \\
\text { Breast } \\
\text { Melanoma } \\
\text { RCC } \\
\text { Gl }\end{array}$ & $\begin{array}{c}86(63.2) \\
16(11.8) \\
11(8.1) \\
7(5.1) \\
16(11.8)\end{array}$ & $\begin{array}{c}79(59.4) \\
16(12.0) \\
12(9.0) \\
11(8.3) \\
15(11.3)\end{array}$ & $\begin{array}{c}70(52.2) \\
16(11.9) \\
12(9.0) \\
11(8.2) \\
25(18.7)\end{array}$ \\
\hline $\begin{array}{l}\text { Extracranial } \\
\text { metastasis, n (\%) }\end{array}$ & $\begin{array}{l}\text { Yes } \\
\text { No }\end{array}$ & $\begin{array}{l}70(51.5) \\
66(48.5)\end{array}$ & $\begin{array}{l}73(54.9) \\
60(45.1)\end{array}$ & $\begin{array}{l}74(55.2) \\
60(44.8)\end{array}$ \\
\hline Number of BM, n (\%) & $\begin{array}{l}1 \\
2 \\
3 \\
\geq 4\end{array}$ & $\begin{array}{c}68(50.0) \\
33(24.3) \\
25(18.4) \\
10(7.4)\end{array}$ & $\begin{array}{l}69(51.9) \\
27(20.3) \\
22(16.5) \\
15(11.3)\end{array}$ & $\begin{array}{l}65(48.5) \\
28(20.9) \\
24(17.9) \\
17(12.7)\end{array}$ \\
\hline $\begin{array}{l}\text { EGFR/ALK status, } \\
\text { n (\%)* }\end{array}$ & $\begin{array}{l}\text { Positive } \\
\text { Negative }\end{array}$ & $\begin{array}{c}6(7.0) \\
80(93.0)\end{array}$ & $\begin{array}{c}9(11.4) \\
70(88.6)\end{array}$ & $\begin{array}{c}4(5.7) \\
66(94.3)\end{array}$ \\
\hline ER/PR status, n (\%)* & $\begin{array}{l}\text { Positive } \\
\text { Negative }\end{array}$ & $\begin{array}{l}10(62.5) \\
6(37.5)\end{array}$ & $\begin{array}{l}4(25.0) \\
12(75)\end{array}$ & $\begin{array}{l}6(37.5) \\
10(62.5)\end{array}$ \\
\hline HER2 status, n (\%)* & $\begin{array}{l}\text { Positive } \\
\text { Negative } \\
\text { Missing }\end{array}$ & $\begin{array}{c}8(50.0) \\
8(50.0) \\
-\end{array}$ & $\begin{array}{c}8(50.0) \\
7(43.8) \\
1(6.3)\end{array}$ & $\begin{array}{c}10(62.5) \\
5(31.3) \\
1(6.3)\end{array}$ \\
\hline BRAF status, n (\%)* & $\begin{array}{l}\text { Positive } \\
\text { Negative } \\
\text { Missing }\end{array}$ & $\begin{array}{c}5(45.5) \\
5(45.5) \\
1(9.1)\end{array}$ & $\begin{array}{c}6(50.0) \\
6(50.0) \\
-\end{array}$ & $\begin{array}{c}4(33.3) \\
8(66.7) \\
-\end{array}$ \\
\hline $\begin{array}{l}\text { Hb, mean (SD)* } \\
\text { Missing, } n(\%)\end{array}$ & & $\begin{array}{l}8.2(0.5) \\
4(57.1)\end{array}$ & $\begin{array}{l}8.5(1.1) \\
3(27.3)\end{array}$ & $\begin{array}{l}7.8(0.8) \\
2(18.2)\end{array}$ \\
\hline
\end{tabular}

BM: brain metastasis, Gl: gastro-intestinal, KPS: Karnofsky Performance Status, NSCLC: non-small cell lung carcinoma, RCC: renal cell carcinoma, SD: standard deviation, SES: socioeconomic status

* Data for relevant primary tumour type 
In accordance with the DAG, the estimation of total effect of SES on survival was achieved by correcting only for the confounder age, resulting in a HR of 0.95 ( $95 \% \mathrm{Cl} 0.84-1.07)$. The direct effect was determined by correcting for age, extracranial metastasis, KPS, number of brain metastases, and primary tumour location. This gave a HR of $0.96(95 \% \mathrm{Cl}$ $0.84-1.10)$. None of the estimated HRs for SES were statistically significant within the imputed dataset. All models conformed to assumptions of linearity and proportional hazards, after the appropriate adjustments were made. This included a 5-knot spline for age, and entering KPS and the number of brain metastases as strata to the regression model for the direct effect.

Table 2 Delivered radiotherapy doses and fractionation

\begin{tabular}{lll}
\hline Type & Fractions and dose & $\mathbf{n}(\%)$ \\
\hline SRS & $1 \times 16 \mathrm{~Gy}$ & $6(1.5 \%)$ \\
& $1 \times 18 \mathrm{GY}$ & $55(13.6 \%)$ \\
& $1 \times 20 \mathrm{~Gy}$ & $93(23.0 \%)$ \\
& $1 \times 21 \mathrm{~Gy}$ & $62(15.3 \%)$ \\
& $1 \times 24 \mathrm{~Gy}$ & $17(4.2 \%)$ \\
FSRT & $3 \times 8 \mathrm{~Gy}$ & $147(36.4 \%)$ \\
& $5 \times 6 \mathrm{~Gy}$ & $18(4.5 \%)$ \\
Other & $10 \times 30 \mathrm{~Gy}$ & $5(1.2 \%)$ \\
\hline
\end{tabular}

FSRT = fractionated radiotherapy; SRS = stereotactic radiosurgery

Unadjusted median survival in the low, middle and high tertile was respectively 10.0, 8.8, and 10.5 months (Table 3). Adjusting to obtain the direct and total effects resulted in similar survival numbers, and non-significant HRs of I.I for middle vs low SES, and 1.0 of high vs low SES. The unadjusted and IPW-adjusted Kaplan Meier survival curves (Figures 2 and 3) both show similar survival for each SES tertile. The latter curve was adjusted with the variables needed to obtain the direct effect, the survival curves for the total effect was similar in shape. The log-rank test of the unadjusted Kaplan Meier plot resulted in a nonsignificant difference, with a chi-square of 0.5 and a p-value of 0.8 . 


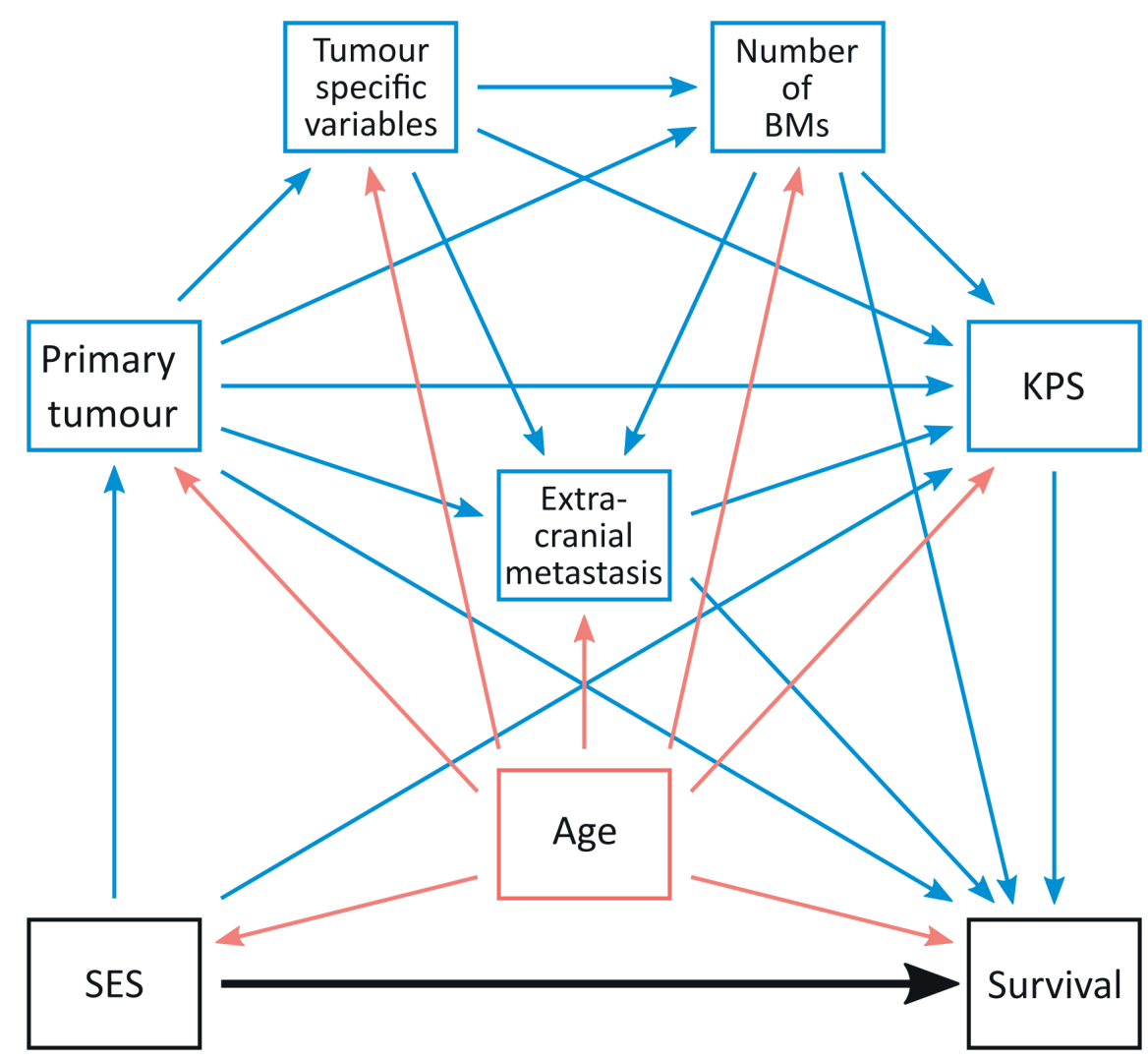

Figure I: Directed acyclic graph (DAG), showing the relation between all collected clinical variables. Shown are the direct relation between the determinant and outcome (black arrow), and the influence of confounders (red) and mediating factors (blue). $\mathrm{BM}=$ brain metastases, KPS = Karnofsky Performance Status, SES = socioeconomic status 
Table 3 Median survival, survival rates and hazard ratios of each SES tertile, taken from the unadjusted, total and direct effects models

\begin{tabular}{|c|c|c|c|c|}
\hline & & \multicolumn{3}{|c|}{ Tertiles } \\
\hline & & $\begin{array}{c}\text { Low SES } \\
{[-2.7-0.2]}\end{array}$ & $\begin{array}{l}\text { Mid SES } \\
{[0.2-0.8]}\end{array}$ & $\begin{array}{l}\text { High SES } \\
{[0.8-2.6]}\end{array}$ \\
\hline \multirow[t]{4}{*}{ Unadjusted } & $\begin{array}{l}\text { Median survival } \\
\text { (months; } 95 \% \mathrm{Cl} \text { ) }\end{array}$ & $10.0(7.4-15.4)$ & $8.8(7.9-12.1)$ & $10.5(7.6-14.8)$ \\
\hline & $\begin{array}{l}\text { I-year survival } \\
\text { (\%; 95\% Cl) }\end{array}$ & $44.9(37.2-54.0)$ & $42.1(34.5-51.4)$ & $46.3(38.6-55.5)$ \\
\hline & $\begin{array}{c}\text { 2-year survival } \\
\text { (\%; } 95 \% \mathrm{Cl})\end{array}$ & $27.6(21.0-36.4)$ & $24.0(17.7-32.5)$ & $25.0(18.6-33.6)$ \\
\hline & $\mathrm{HR}(95 \% \mathrm{CI})$ & (reference) & I.I (0.8-1.4) & $1.0(0.8-1.3)$ \\
\hline \multirow[t]{4}{*}{ Total effect } & $\begin{array}{l}\text { Median survival } \\
\text { (months; } 95 \% \mathrm{Cl} \text { ) }\end{array}$ & $9.9(7.8-13.6)$ & $8.8(7.7-12.0)$ & $10.7(7.8-15.5)$ \\
\hline & $\begin{array}{l}\text { I-year survival } \\
\text { (\%; 95\% Cl) }\end{array}$ & $44.5(36.9-53.6)$ & $41.0(33.4-50.3)$ & $47.5(39.8-56.8)$ \\
\hline & $\begin{array}{c}\text { 2-year survival } \\
\text { (\%; } 95 \% \mathrm{Cl})\end{array}$ & $27.4(20.9-36.1)$ & $23.1(16.9-31.6)$ & $25.7(19.2-34.3)$ \\
\hline & $\mathrm{HR}(95 \% \mathrm{Cl})$ & (reference) & I.I (0.9-1.4) & $1.0(0.8-1.3)$ \\
\hline \multirow[t]{4}{*}{$\begin{array}{l}\text { Direct } \\
\text { effect }\end{array}$} & $\begin{array}{l}\text { Median survival } \\
\text { (months; } 95 \% \mathrm{Cl} \text { ) }\end{array}$ & $9.6(7.4-13.4)$ & $8.8(7.6-12.1)$ & $10.7(7.8-15.5)$ \\
\hline & $\begin{array}{l}\text { I-year survival } \\
(\% ; 95 \% \mathrm{CI})\end{array}$ & $44.0(36.4-53.2)$ & $41.6(34.0-50.8)$ & $47.5(39.8-56.8)$ \\
\hline & $\begin{array}{c}\text { 2-year survival } \\
(\% ; 95 \% \mathrm{Cl})\end{array}$ & $27.0(20.4-35.6)$ & $23.7(17.4-32.1)$ & $26.2(19.7-34.9)$ \\
\hline & HR $(95 \% \mathrm{CI})$ & (reference) & I.I (0.9-1.5) & $1.0(0.7-1.3)$ \\
\hline
\end{tabular}


Unadjusted survival curves

SES tertiles + low + mid + high
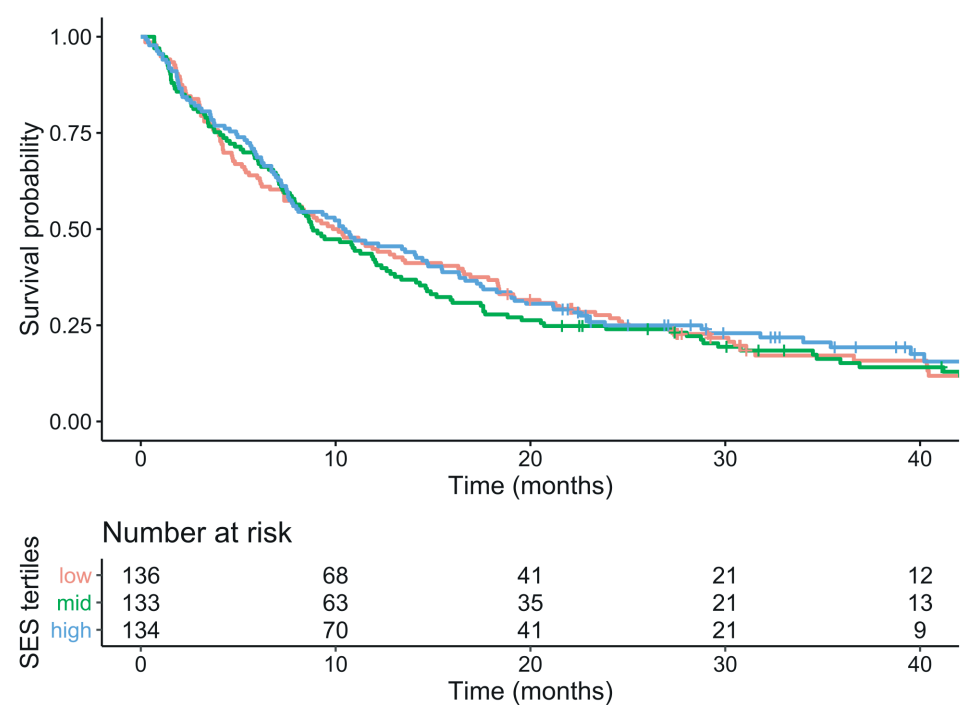

Figure 2: Survival curves comparing the SES tertiles, unadjusted for other clinical variables (logrank test $p=0.8$ )

\section{Adjusted survival curves}
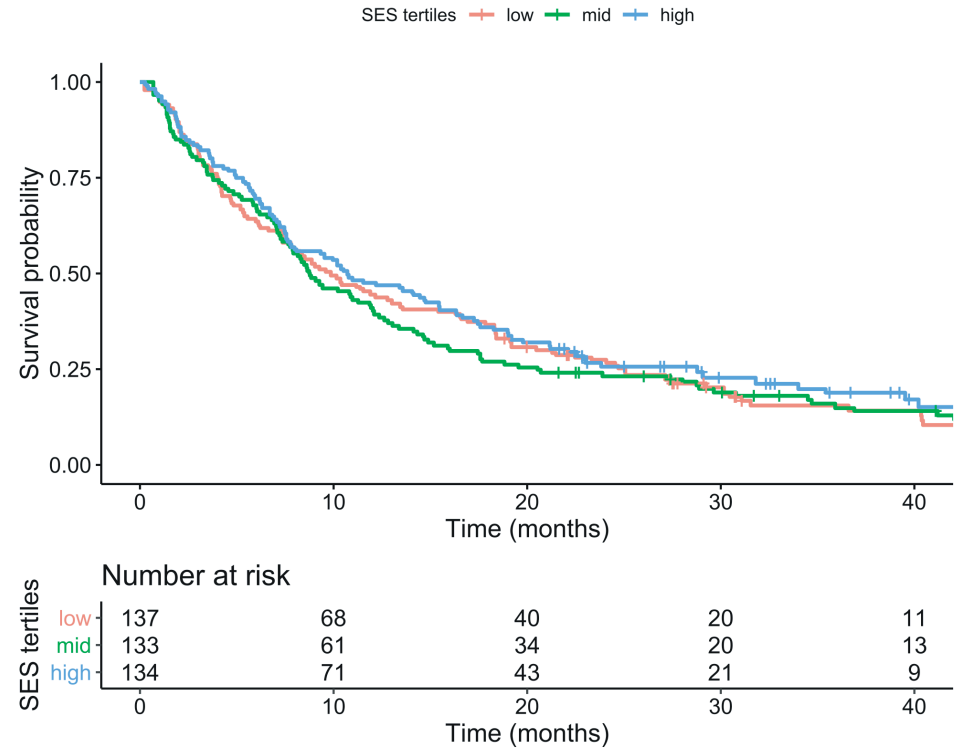

Figure 3: Survival curves comparing the SES tertiles, adjusted with inverse probability weighting for the clinical variables needed to obtain the direct effect 


\section{DISCUSSION}

We have found that socioeconomic status (SES) is not related to overall survival in patients with brain metastases referred for and treated with stereotactic radiosurgery in a tertiary radiotherapy facility in the Netherlands. Both in unadjusted analysis as well as when adjusting for other clinical factors, we found a non-significant relation between SES and survival, with HRs and confidence intervals suggesting no relevant clinical impact either. The survival curves lead to a similar conclusion, showing no clear differences in survival patterns between the SES tertiles.

This not the first study into the relation between SES and survival in brain metastasis patients. Previous studies have been performed, with differing results.

Ten Berge et al studied a cohort of II29 Dutch patients with synchronously diagnosed brain metastases and NSCLC treated with Gamma Knife (GK) radiosurgery. ${ }^{15}$ They found a significant relation between SES (in tertiles, based on the same SCP database as the present study) and survival, with HRs of $0.9(95 \% \mathrm{Cl} 0.7-0.995)$ and $0.8(95 \% \mathrm{Cl} 0.7$ 0.9) for the intermediate and high SES groups, compared to the low group. Analysis was corrected for a large number of factors, including age, gender, and primary tumour stage and adenocarcinoma histology. Their observed point estimates are close to the HRs we found (0.95 and 0.96), and thereby are close to I. This could lead one to argue that, even though there is a significant effect, its clinical impact is limited. The authors themselves did not reflect on this effect of SES on survival, as their primary aim was to study whether there are factors that influence referral patterns in these patients. They found that patients had increased odds to be referred for GK if they were below 60 years of age and had limited lymphatic spread (i.e. lower $\mathrm{N}$-stage). This (aside from the more selective study population) might partly explain the difference to our results, although SES did not affect the referral pattern.

A cohort of 737 patients undergoing SRS for brain metastases from the most common primary tumour sites in the United States was studied by Alphonse-Sullivan et al.' ${ }^{2}$ They examined four factors reflecting a patient's SES: median income (based on postal code), race, rural or urban residence, and health insurance status. They found a significant result for median income, but mention there is no increasing or decreasing trend across the income groups. None of the other factors was associated with survival. The absence of income data in our cohort means we cannot compare these results to ours.

A Chinese study in 209 NSCLC patients with multiple brain metastases found a significant effect of a patient's "cultural background" on survival. ${ }^{14}$ This metric was composed of 
socioeconomic status, level of education, understanding of the disease, and the degree of care and support they received from family members. It is unclear how these factors were measured or defined, meaning our results cannot be compared to theirs. They concluded that, compared to the highest level, the two lowest cultural background groups had significantly poorer survival.

Finally, two studies examined the effect of SES of cohorts including, but not limited to, brain metastasis patients. They did not find any relation between SES and survival.13,25

Survival is not the only outcome of interest in studying the effect of SES in brain metastasis patients. Along with the previously mentioned study by Ten Berge et al., two other recent studies have looked at the effect of SES on treatment decisions, ${ }^{26,27}$ which may - in turn impact survival for the total population of patients with brain metastases. The first study found no relation between referral patterns, but the latter two have found that patients within a higher SES quartile are more likely to receive SRS.

There are some limitations to this study. Firstly, this is relatively small cohort, especially compared to that of Ten Berge et al. This might mean that our sample size is too small to find a significant relationship between SES and survival. However, the HRs found both in our study and that by Ten Berge et al. suggest limited clinical relevance, even if a significant effect is present.

Secondly, our SES score was based on a patient's postal code, meaning that there may be imprecisions in this metric. Should a patient be an outlier in terms of education, income or unemployment, this would not be reflected their assigned SES score. This means the SES data we used for analysis can be either too high or too low, but is always based on an average score of a patients' neighbourhood. This could have led to an underestimation of the observed effect. There is no consensus as to which way of determining SES is the best in cancer-related survival studies, ${ }^{28}$ which may also explain the variance in previously published studies. Different methods may result in contrasting conclusions, meaning the SES scoring type should be chosen carefully. Unfortunately, the currently used SES score is the only one available to us, so a comparison between different scoring metrics cannot be performed. However, we have used the same SES scores as Ten Berge et al., which increases comparability of the outcomes.

As with most retrospective studies, we were faced with missing data. Since the rate of missing data was relatively low, and as we used multiple imputation (currently the best method available to minimize bias), we feel that the effects of these missing data were limited. 
Finally, and perhaps most importantly, selection bias plays a role. We have selected only those patients receiving SRS or FSRT for the 5 most common primary sites. This excludes a number of brain metastases patients, particularly those receiving whole brain radiotherapy, or those not receiving radiation treatment at all. This means that we do not have a representative cohort that allows us to study the effect of SES of the entire brain metastasis population, but only on the SRS/FSRT-treated subgroup. As mentioned above, referral patterns for SRS can be affected by SES, which in turn has an effect on survival. This means that treatment referral may be an additional mediator between SES and survival, but one we cannot correct for.

Our results warrant several directions for future studies. In order to conclusively say that SES does not affect survival, and in light of our major two limitations, a bigger cohort of brain metastasis patients should be selected for whom all relevant SES factors are recorded. As this includes annual income and employment status, which is not routinely recorded in clinical practice, this would require prospective data collection. This cohort should also include those patients who did not receive SRS or FSRT, to reflect the entire brain metastasis population. Additionally, we have seen that difference in local or national healthcare policies might influence differences seen between SES groups. A large-scale cohort comprised of several countries representing different healthcare systems (e.g. nationalized vs private healthcare, obligatory health insurance yes or no) could lead to conclusions on the level of equality of treatment outcomes in these systems.

\section{CONCLUSION}

We have studied the effect of socioeconomic status on survival in a cohort of brain metastases patients receiving either SRS or FSRT. When correcting for clinical variables, we found no significant relationship between the estimated SES and survival, with HRs suggesting limited clinical impact. Although our study has certain limitations, including selection bias due to treatment referral patterns, the results suggest that patients' survival outcomes after contemporary cancer treatment are unrelated to their employment status, education status and annual income.

\section{ACKNOWLEDGMENTS}

Special thanks to Gery Tijsseling for providing data management for this study. 


\section{REFERENCES}

I. Berghoff AS, Schur S, Füreder LM, et al. Descriptive statistical analysis of a real life cohort of 2419 patients with brain metastases of solid cancers. ESMO open. 2016;1(2):e000024. doi:10.1136/ esmoopen-2015-000024

2. Cagney DN, Martin AM, Catalano PJ, et al. Incidence and prognosis of patients with brain metastases at diagnosis of systemic malignancy: a population-based study. Neuro-Oncology. 2017;19(| I):151 |-|521. doi:10.1093/neuonc/nox077

3. Andrews DW, Scott CB, Sperduto PW, et al. Whole brain radiation therapy with or without stereotactic radiosurgery boost for patients with one to three brain metastases: phase III results of the RTOG 9508 randomised trial. Lancet (London, England). 2004;363(9422):1665-1672. doi:10.1016/ SOl40-6736(04)16250-8

4. Hasegawa T, Kondziolka D, Flickinger JC, Germanwala A, Lunsford LD. Brain Metastases Treated with Radiosurgery Alone: An Alternative to Whole Brain Radiotherapy? Neurosurgery. 2003;52(6):I3181326. doi:I0.1227/0I.NEU.0000064569.189|4.DE

5. Nayak L, Lee EQ, Wen PY. Epidemiology of Brain Metastases. Current Oncology Reports. 20 I2; 14(I):4854. doi:I0.1007/s|1912-011-0203-y

6. Kogevinas M, Porta M. Socioeconomic differences in cancer survival: a review of the evidence. IARC scientific publications. 1997;(I38): I77-206.

7. Cella DF, Orav EJ, Kornblith AB, et al. Socioeconomic status and cancer survival. Journal of clinical oncology: official journal of the American Society of Clinical Oncology. 1991;9(8):1500-1509. doi:10.1200/ JCO.1991.9.8.1500

8. Schrijvers CT, Mackenbach JP. Cancer patient survival by socioeconomic status in seven countries: a review for six common cancer sites [corrected]. Journal of epidemiology and community health. 1994;48(5):44I-446.

9. Ward E, Jemal A, Cokkinides V, et al. Cancer Disparities by Race/Ethnicity and Socioeconomic Status. CA: A Cancer Journal for Clinicians. 2004;54(2):78-93. doi:I0.3322/canjclin.54.2.78

10. Institute of Medicine. Unequal Treatment: Confronting Racial and Ethnic Disparities in Health Care. (Smedley BD, Stith AY, Nelson AR, eds.). The National Academies Press; 2003. doi: 10.17226/I2875

II. Freeman HP. Commentary on the meaning of race in science and society. Cancer epidemiology, biomarkers \& prevention : a publication of the American Association for Cancer Research, cosponsored by the American Society of Preventive Oncology. 2003;12(3):232s-236s.

12. Alphonse-Sullivan N, Taksler GB, Lycan T, et al. Sociodemographic predictors of patients with brain metastases treated with stereotactic radiosurgery. Oncotarget. 2017;8(60):101005-101011. doi:10.18632/oncotarget.2229|

13. Williams M, Woolf D, Dickson J, Hughes R, Maher J, Mount Vernon Cancer Centre. Routine clinical data predict survival after palliative radiotherapy: an opportunity to improve end of life care. Clinical oncology (Royal College of Radiologists (Great Britain)). 2013;25(II):668-673. doi: 10. I016/j. clon.2013.06.003

14. Gong X, Zhou D, Liang S, Zhou C. Analyses of prognostic factors in cases of non-small cell lung cancer with multiple brain metastases. OncoTargets and therapy. 2016;9:977-983. doi:10.2147/OTT. S94524

15. Ten Berge DMHJ, Aarts MJ, Hanssens PEJ, Beute GN, Aerts JGJ V, Kloover JS. Referral Patterns and Outcome of Patients With Synchronous Brain Metastases From Non-small Cell Lung Cancer Treated With Gamma Knife Radiosurgery in a Third-Line Treatment Centre in The Netherlands - A Retrospective Analysis. Clinical oncology (Royal College of Radiologists (Great Britain)). 2020;32(I):52-59. doi:10.1016/j.clon.2019.07.004

16. Sociaal en Cultureel Planbureau. Statusscores. Published 2018. https://www.scp.nl/Onderzoek/ Lopend_onderzoek/A_Z_alle_lopende_onderzoeken/Statusscores

17. Moons KGM, Donders RART, Stijnen T, Harrell FE. Using the outcome for imputation of missing predictor values was preferred. Journal of clinical epidemiology. 2006;59(I0):1092-1 I0I. doi:10.1016/j. jclinepi.2006.01.009

18. van Buren S. Flexible Imputation of Missing Data. Second Edi. CRC press/Chapman \& Hall; 2018. 
19. Schemper M, Smith TL. A note on quantifying follow-up in studies of failure time. Controlled Clinical Trials. 1996;17(4):343-346. doi:10.1016/0197-2456(96)00075-X

20. Textor J, van der Zander B, Gilthorpe MS, Liskiewicz M, Ellison GT. Robust causal inference using directed acyclic graphs: the R package "dagitty". International journal of epidemiology. 2016;45(6): 1887|894. doi:10.1093/ije/dyw34|

21. Robins JM, Hernán MÁ, Brumback B. Marginal Structural Models and Causal Inference in Epidemiology. Epidemiology. 2000; II(5):550-560. doi:10.1097/00001648-200009000-000II

22. Cole SR, Hernan MA. Constructing Inverse Probability Weights for Marginal Structural Models. American Journal of Epidemiology. 2008;168(6):656-664. doi:10.1093/aje/kwnI64

23. Rubin DB, ed. Multiple Imputation for Nonresponse in Surveys. John Wiley \& Sons, Inc.; 1987. doi:I0.1002/9780470316696

24. R Core Team. R: A language and environment for statistical computing. R Foundation for Statistical Computing. Published online 2017.

25. Klar N, Rosenzweig M, Diergaarde B, Brufsky A. Features Associated With Long-Term Survival in Patients With Metastatic Breast Cancer. Clinical breast cancer. 2019;19(4):304-310. doi:10.1016/j. clbc.2019.01.014

26. Halasz LM, Weeks JC, Neville BA, Taback N, Punglia RS. Use of Stereotactic Radiosurgery for Brain Metastases From Non-Small Cell Lung Cancer in the United States. International Journal of Radiation Oncology*Biology*Physics. 2013;85(2):el09-el 16. doi:10.1016/j.ijrobp.2012.08.007

27. Barbour AB, Jacobs CD, Williamson H, et al. Radiation Therapy Practice Patterns for Brain Metastases in the United States in the Stereotactic Radiosurgery Era. Advances in Radiation Oncology. 2020;5(I):4352. doi:10.1016/j.adro.2019.07.012

28. Zhang-Salomons J, Qian H, Holowaty E, Mackillop WJ. Associations between socioeconomic status and cancer survival: choice of SES indicator may affect results. Annals of epidemiology. 2006;16(7):521 528. doi:10.1016/j.annepidem.2005.10.002 


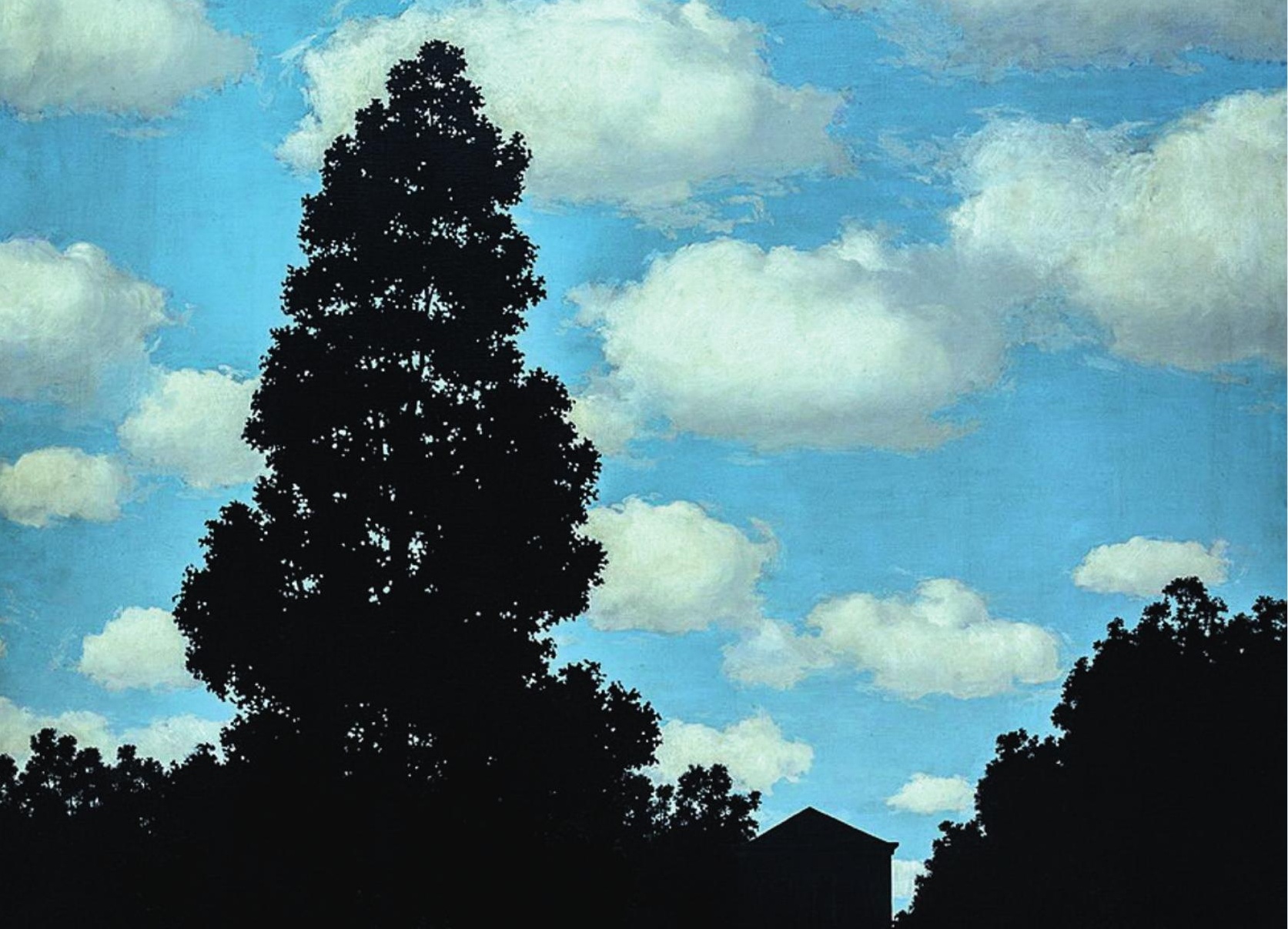

4t

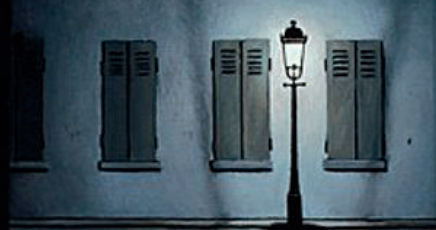




\section{Chapter 9}

Clinical outcomes, imaging and quality of life in patients with brain metastases treated with radiotherapy: design and first results of the COIMBRA prospective cohort

S.H.J. Nagtegaal, E.E. van Grinsven, E.J. Smid, C.E. Kleynen, E. Seravalli, T.J. Snijders, H.M. Verkooijen, J.J.C. Verhoeff 


\section{ABSTRACT}

\section{Purpose}

In order to provide an infrastructure to facilitate inclusion of clinical trials, the Trials within Cohorts (TwiCs) design was created. Patients are included in a prospective observational cohort and give consent to undergo randomization for future trials. We have implemented this design in COIMBRA, a Cohort for patient-reported Outcomes, Imaging and trial inclusion in Metastatic BRAin disease. We present the design of COIMBRA, as well as selected baseline characteristics.

\section{Methods}

Clinical data are prospectively collected. In addition, participants can consent to three further study procedures: I) collection of patient-reported outcomes (PROs) by filling out questionnaires, 2) undergoing additional research MRIs, and 3) undergoing randomization for future trials as part of the TwiCs design. The collected PROs include the EORTC QLQC30 and BN20, Cognitive Failures Questionnaire (CFQ), Hospital Anxiety and Depression Scale (HADS), and Multidimensional Fatigue Inventory (MFI).

\section{Results}

After more than a year of active recruitment, III (73\%) of I53 eligible patients gave informed consent and were enrolled in the cohort. A total of 75 patients (67.6\%) consented to undergo future randomization in TwiCs. Consent for undergoing additional MRIs and filling in the questionnaires was given by $74(66.7 \%)$ and 86 (77.5\%) patients, respectively. PROs indicated overall quality of life comparable to the general cancer and brain tumor population.

\section{Conclusions}

The first year of COIMBRA has shown that patients with brain metastases are willing to participate in a cohort from which TwiCs can be performed. This gives us the opportunity to efficiently recruit patients for future randomized trials. 


\section{INTRODUCTION}

Of all patients diagnosed with cancer, 20-40\% will develop brain metastases (BMs). ${ }^{1-3}$ The incidence is expected to increase with more effective treatments for the primary tumors, thereby improving survival and thus increasing dissemination time of tumor to the brain. ${ }^{2,4}$ BMs most frequently originate from lung cancer, breast cancer and melanoma, ${ }^{2,4-6}$ but in up to $14 \%$ of patients the metastases are of unknown origin. 5,6 Median survival of BMs ranges from 2 to 12 months, and is dependent on primary tumor location, Karnofsky Performance Scale (KPS) score, age, number of BMs, presence of metastases elsewhere and primary tumor control status. ${ }^{3,7}$

Many new treatments are continuously being developed, all of which warrant multiple trials to assess safety, determine the efficacy and optimize protocols. The gold standard of clinical trials is the randomized controlled trial (RCT). However, RCTs assessing novel oncological treatments are faced with recurrent problems, such as failure to reach target inclusion, highly selective study populations and low generalizability of results. To help address these issues, the concept of trials within cohorts (TwiCs) was developed. ${ }^{8,9}$

We designed a cohort for patients with BMs according to the TwiCs principles: the "Cohort for patient-reported Outcomes, Imaging and trial inclusion in Metastatic BRAin disease" (COIMBRA). This cohort has two goals: I) collection of prospective data on clinical factors and patient-reported outcomes before and after radiotherapy for BMs, and 2) creation of an infrastructure in which both randomized controlled clinical trials and imaging studies can be conducted.

In this paper, we describe the study design of COIMBRA, and present baseline clinical characteristics and PROs after 19 months of recruitment.

\section{METHODS}

\section{Enrollment}

Patients are asked to participate in the COIMBRA study after referral to the radiotherapy (RT) department of the UMC Utrecht for either I) radiotherapy for histologically proven or radiologically suspect brain metastases, or 2) prophylactic cranial irradiation (PCl) for small-cell lung cancer. Exclusion criteria are: age below 18 years; developmental, psychiatric, or cognitive disorders that hinder the patient's ability to understand the informed consent procedure; and inability to understand the Dutch language. 
Prior to the first visit with their radiation oncologist, patients receive written information on COIMBRA. On the day of the appointment at the department, a visit with a researcher or research assistant is scheduled. The cohort is further clarified, any questions are answered, and the written informed consent is completed. All participants are required to give informed consent for the collection and use of their clinical data in order to participate in COIMBRA. In addition, patients are asked to opt in or out of three other study procedures: I) filling out PRO questionnaires, 2) undergoing additional MRIs, and 3) future randomization for clinical trials.

The protocol for COIMBRA was approved by the local Institutional Review and Ethics Board, with number 18-642.

\section{Clinical data collection}

Data on patient demographics, primary tumor and BM characteristics, received treatments and clinical outcomes are prospectively collected for all included patients. Sources of this information are electronic patient records, imaging and referral letters.

Demographic data include age at the start of radiotherapy for BMs, sex, body mass index and KPS. Primary tumor characteristics are tumor location, histopathological subtype, presence of extracranial metastases and date of first diagnosis. Data on BMs include total number present, date of diagnosis and presence of neurological symptoms. Collected treatment data include type, dose and fractionation of cranial RT, and whether patients underwent neurosurgical excision (including extent of resection) and/or systemic treatments. Clinical outcomes are survival and tumor recurrence, as assessed by a specialized radiologist from routine follow-up imaging.

\section{Patient-reported outcomes}

Data on PROs are collected with validated questionnaires. They are sent to the patient before the start of RT, after I month, after 3 months, and once per 3 months from then on. Participants have the choice of filling in paper questionnaires and returning them via mail, or submitting them electronically using a digital platform.

Questionnaires were chosen to collect outcomes relevant to the diagnosis of brain metastases: general health-related quality of life (European Organization for Research and Treatment of Cancer quality of life core questionnaire (EORTC QLQ-C30), with the brain specific module BN20; EQ-5D-3L), cognitive symptoms (Cognitive Failures Questionnaire (CFQ)), anxiety and depression (Hospital Anxiety and Depression Scale; HADS), fatigue (Multidimensional Fatigue Inventory; MFI) and level of physical exercise (Short QUestionnaire to ASsess Health enhancing physical activity; SQUASH). 
Additionally, two questionnaires are used once at baseline to collect data on patients' personality and coping style: the NEO-Five Factor Inventory (NEO-FFI) and the Utrecht Coping List (UCL).

\section{Additional imaging}

Experimental MRI sequences not routinely made during regular clinical imaging can be performed in patients consenting to undergo additional imaging. These are always made consecutively to a regularly scheduled clinical MRI at the department, making sure that the additional burden to the patient is as limited as possible.

\section{Future randomization for TwiCs}

The basis of the TwiCs approach (also known as the cohort multiple Randomized Controlled Trial, cmRCT) is creating an observational prospective cohort, consisting of patients with the same condition of interest undergoing standard treatment (Figure I).

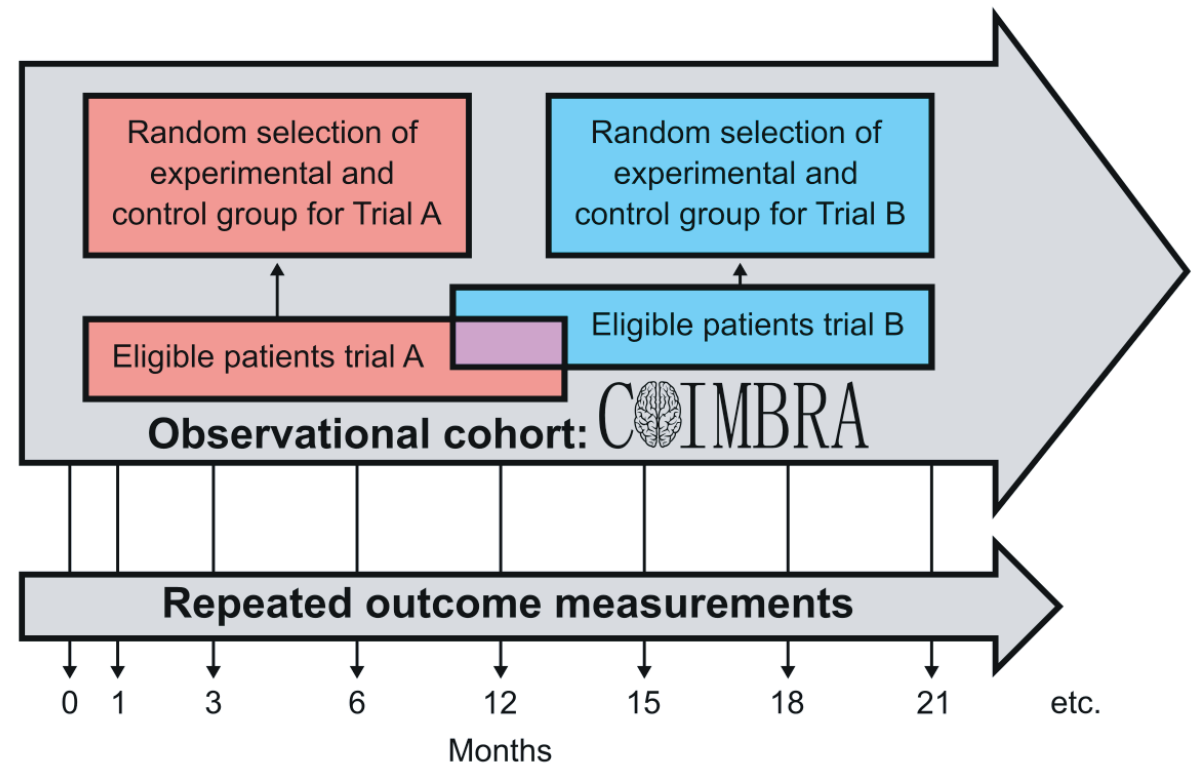

Figure I The Trials within Cohorts (TwiCs) design. A large observational cohort with the condition of interest is recruited. Participants' clinical and self-reported outcomes are regularly measured. For each randomized trial a sub-cohort of eligible patient is identified. Patients from the sub-cohort are randomly selected to undergo the intervention. Their outcomes are then compared to the other patients in the sub-cohort who undergo standard care. This process repeats for each new TwiC that is conducted; patients may be eligible for multiple trials. Figure adapted from Relton et al. ${ }^{8}$ 
When a new intervention is ready for formal evaluation, eligible patients within the observational cohort are identified. From this so-called sub-cohort, patients are randomized into either undergoing the intervention, or receiving standard treatment. Only those selected to undergo the intervention of interest will then be asked to participate in the trial. Should they wish to participate, additional written informed consent for that trial will be obtained. Should they refuse, they will receive standard care. Those selected for the control arm will also undergo standard care, but will not be informed on serving as control arm the trial. This process is called staged informed consent: patients initially consent to undergo randomization for future studies, and at a later time consent to participate in the intervention arm of a new trial. ${ }^{8}$

Outcomes of the selected patients receiving the new intervention will be compared to the outcomes of the non-selected patients receiving the standard treatment. Within this cohort, the same process can be simultaneously performed for other interventions. Advantages of the TwiCs design include on-going information on the natural history of the condition and standard treatment, the ability to facilitate multiple simultaneous randomized evaluations, the improved comparability between trials and the patient-centered informed consent procedure.

\section{Statistical analysis}

In this paper, we present a selection of the most relevant collected baseline characteristics and PROs. Continuous variables are reported as either mean (with standard deviations: SD) or median (with interquartile range: IQR), depending on the normality of their distributions. PROs are summarized in domains, according to their scoring manuals. Outcomes of PROs were compared to reference values and normative data (which were available for the EORTC QLQ-C30, EQ-5D, and HADS ${ }^{10-12}$ ), or data gathered from healthy or cancer populations. ${ }^{13-17}$ 


\section{RESULTS}

\section{Inclusion and consent}

In total, 211 patients were eligible for study inclusion in the period April 2019 - August 2020 (Figure 2). Of these patients, 153 were asked to participate in the cohort, and III (72.5\%) gave written informed consent. Due to national measures to prevent the spread of coronavirus disease (COVID-19), study recruitment was halted between March and June 2020; in this period 32 eligible patients were not approached to participate in COIMBRA. A total of 86 patients (77.5\%) consented to filling in PRO questionnaires. Consent for undergoing additional MRIs and future randomization was given by 74 (66.7\%) and 75 (67.6\%) patients, respectively.

\section{Clinical data}

The mean age at baseline of the entire cohort was 63 years (sd II, range 32 - 86), with males representing $51.4 \%$ of participants (Table I). In 91 cases (82.0\%), patients were referred for radiotherapy of newly discovered brain metastases. Lung carcinoma was the tumor of origin in the majority of cases (58.6\%), and an even larger proportion of patients received stereotactic radiosurgery (88.8\%). The characteristics of the patients who consented to collection of PROs and future randomization (Table I) do not show large differences compared to those of the entire cohort.

\section{Questionnaires}

Questionnaire return rates among patients consenting to collection of PROs were 70.9\% at baseline (61/86). After that, return rates varied between 39.0 and 64.7 percent (Supplementary Table I). Comparing the QLQ-C30 to reference values shows that, while having lower performance scores than the general population, the scores from the COIMBRA cohort are similar to those of cancer and brain tumor patients (Figure 3). The same is seen for EQ-5D, CFQ, HADS and MFI; COIMBRA participants report worse outcomes than the healthy population, but have performance and symptom scores comparable to patients with cancer (Table 2). The only difference is seen in the CFQ, where patients from our cohort report more symptoms than those in healthy and cancer populations. 
Table I Baseline clinical variables for the entire cohort, and for patients who gave consent for PRO collection and future randomization

\begin{tabular}{|c|c|c|c|c|}
\hline & & $\begin{array}{c}\text { Total } \\
\text { cohort } \\
n=|I|\end{array}$ & $\begin{array}{l}\text { Consent for } \\
\text { PROs } \\
n=86\end{array}$ & $\begin{array}{c}\text { Consent for } \\
\text { randomization } \\
n=75\end{array}$ \\
\hline \multirow[t]{2}{*}{ Sex, n (\%) } & Male & $57(51.4)$ & $42(48.8)$ & $37(49.3)$ \\
\hline & Female & $54(48.6)$ & $44(51.2)$ & $38(50.7)$ \\
\hline Age, mean (sd) & & $63(11)$ & $62(10)$ & $62(11)$ \\
\hline \multirow[t]{2}{*}{ Newly discovered BM, n (\%) } & Yes & $91(82.0)$ & $68(79.1)$ & $57(76.0)$ \\
\hline & No & $20(18.0)$ & $18(20.9)$ & $18(24.0)$ \\
\hline \multirow[t]{6}{*}{ Primary tumour, n (\%) } & Lung & $65(58.6)$ & $46(53.5)$ & $38(50.7)$ \\
\hline & Breast & $19(17.1)$ & $15(17.4)$ & $14(18.7)$ \\
\hline & Melanoma & $13(11.7)$ & II (12.8) & $10(13.3)$ \\
\hline & Colon & $5(4.5)$ & $4(4.7)$ & $3(4.0)$ \\
\hline & Kidney & $4(3.6)$ & $2(2.3)$ & $3(4.0)$ \\
\hline & Other/Unknown & $8(7.2)$ & $8(9.3)$ & $7(9.3)$ \\
\hline \multirow[t]{2}{*}{ Extracranial metastasis, n (\%) } & Yes & $41(36.9)$ & $30(34.9)$ & $27(36.0)$ \\
\hline & No & $70(63.1)$ & $56(65.1)$ & $48(64.0)$ \\
\hline \multirow[t]{6}{*}{ Number of BMs, n (\%) } & 0 & $2(1.8)$ & I (l.2) & - \\
\hline & । & $46(4 \mid .4)$ & $34(39.5)$ & 31 (4l.3) \\
\hline & 2 & $19(17.1)$ & $15(17.4)$ & $10(13.3)$ \\
\hline & 3 & $13(11.7)$ & $10(11.6)$ & $9(12.0)$ \\
\hline & $4-9$ & $18(16.2)$ & $14(16.3)$ & $13(17.3)$ \\
\hline & $10+$ & $13(11.7)$ & $12(14.0)$ & $12(16.0)$ \\
\hline \multirow[t]{5}{*}{ KPS, n (\%) } & $0-50$ & $7(6.3)$ & $3(3.5)$ & $4(5.3)$ \\
\hline & $60-70$ & $31(27.9)$ & $24(27.9)$ & II (14.7) \\
\hline & $80-90$ & $63(56.8)$ & $51(59.3)$ & $18(24.0)$ \\
\hline & 100 & $6(5.4)$ & $5(5.8)$ & I (2.8) \\
\hline & Missing & $4(3.6)$ & $3(3.5)$ & $2(5.6)$ \\
\hline \multirow[t]{2}{*}{ RT, n (\%) } & Yes & $107(96.4)$ & $82(95.3)$ & $7 \mid(94.7)$ \\
\hline & No & $4(3.6)$ & $4(4.7)$ & $4(5.3)$ \\
\hline \multirow[t]{3}{*}{ RT type, n (\%) } & SRS & $95(88.8)$ & $73(84.9)$ & $63(84.0)$ \\
\hline & WBRT & $10(9.3)$ & $8(9.3)$ & $8(10.7)$ \\
\hline & $\mathrm{PCl}$ & $2(1.9)$ & । ( 1.2$)$ & - \\
\hline Maximum prescribed & Ix24 Gy & $29(27.1)$ & $17(19.8)$ & $17(22.7)$ \\
\hline \multirow[t]{4}{*}{ fractionation and dose, n (\%) } & $|x 2| \mathrm{Gy}$ & $29(27.1)$ & $26(30.2)$ & $19(25.3)$ \\
\hline & Ix|8 Gy & $25(23.4)$ & $20(23.3)$ & $18(24.0)$ \\
\hline & Ix16 Gy & $4(3.7)$ & $2(2.3)$ & $2(2.7)$ \\
\hline & IxI5 Gy & $8(7.5)$ & $8(9.3)$ & $7(9.3)$ \\
\hline
\end{tabular}


Table I Continued.

\begin{tabular}{llccc}
\hline & & $\begin{array}{c}\text { Total } \\
\text { cohort } \\
\mathbf{n = 1 I I}\end{array}$ & $\begin{array}{c}\text { Consent for } \\
\text { PROs } \\
\mathbf{n = 8 6}\end{array}$ & $\begin{array}{c}\text { Consent for } \\
\text { randomization } \\
\mathbf{n = 7 5}\end{array}$ \\
\hline \multirow{3}{*}{ Systemic therapy, n (\%) } & Yx4 Gy & $12(11.2)$ & $9(10.5)$ & $8(10.7)$ \\
& Yes & $60(54.1)$ & $49(57.0)$ & $47(62.7)$ \\
BM resection, n (\%) & No & $51(45.9)$ & $37(43.0)$ & $28(37.3)$ \\
& Yes & $31(27.9)$ & $25(29.1)$ & $20(26.7)$ \\
& No & $80(72.1)$ & $61(70.9)$ & $55(73.3)$ \\
\hline
\end{tabular}

$\mathrm{BM}=$ brain metastasis; $\mathrm{KPS}=$ Karnofsky Performance Scale; $\mathrm{PCl}$ = prophylactic cranial irradiation; $\mathrm{PRO}=$ Patient-reported outcome; $\mathrm{RT}=$ Radiotherapy; $\mathrm{SRS}=$ stereotactic radiosurgery; WBRT $=$ whole-brain radiotherapy

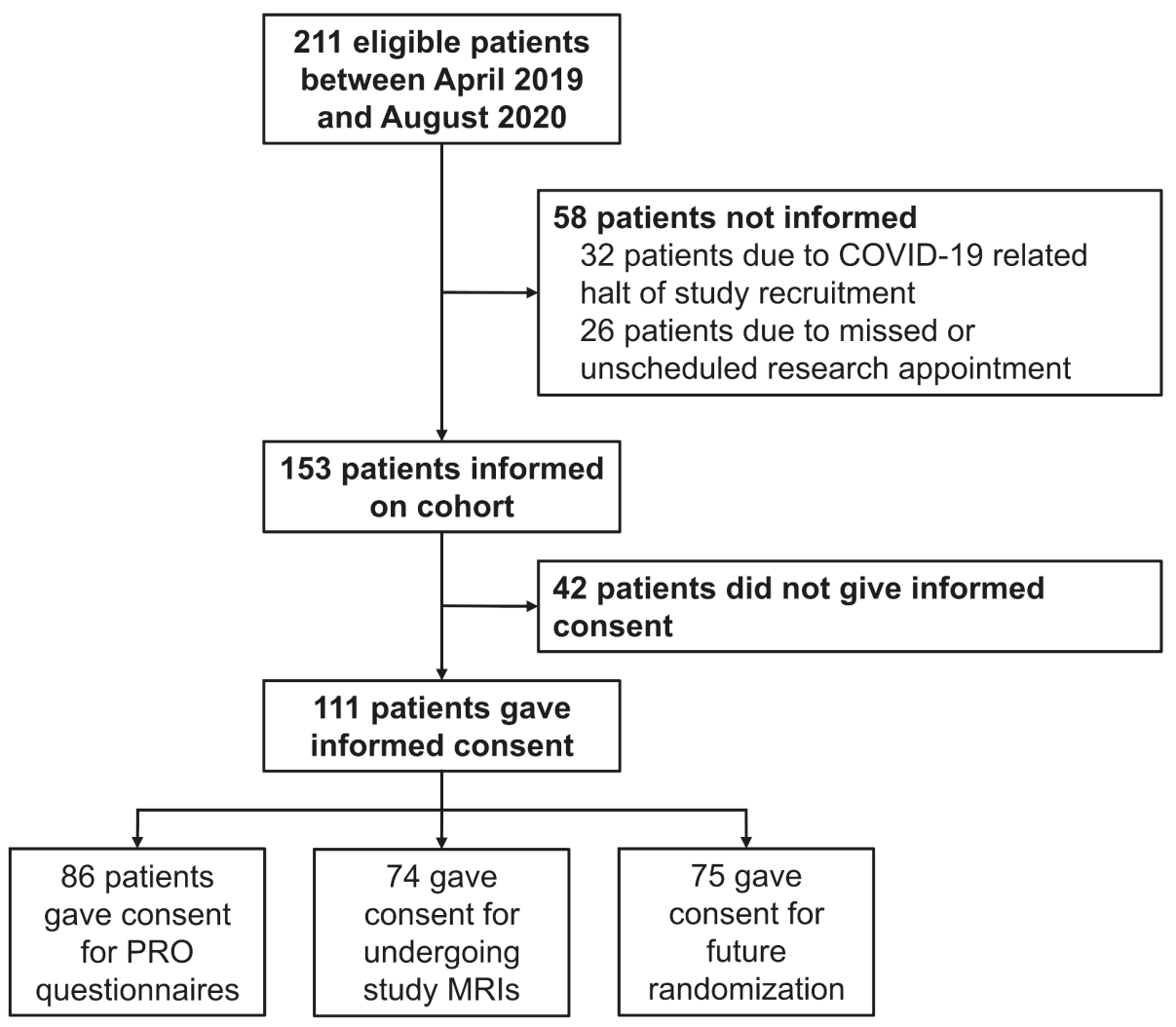

Figure 2 Flow-chart of patient inclusion in COIMBRA. Due to national measures to prevent the spread of coronavirus disease (COVID-19), study recruitment was halted, meaning 32 eligible patients were not approached to participate in the COIMBRA trial. PRO = patient reported outcomes 


\section{Comparison of median QLQ-C30 scores}

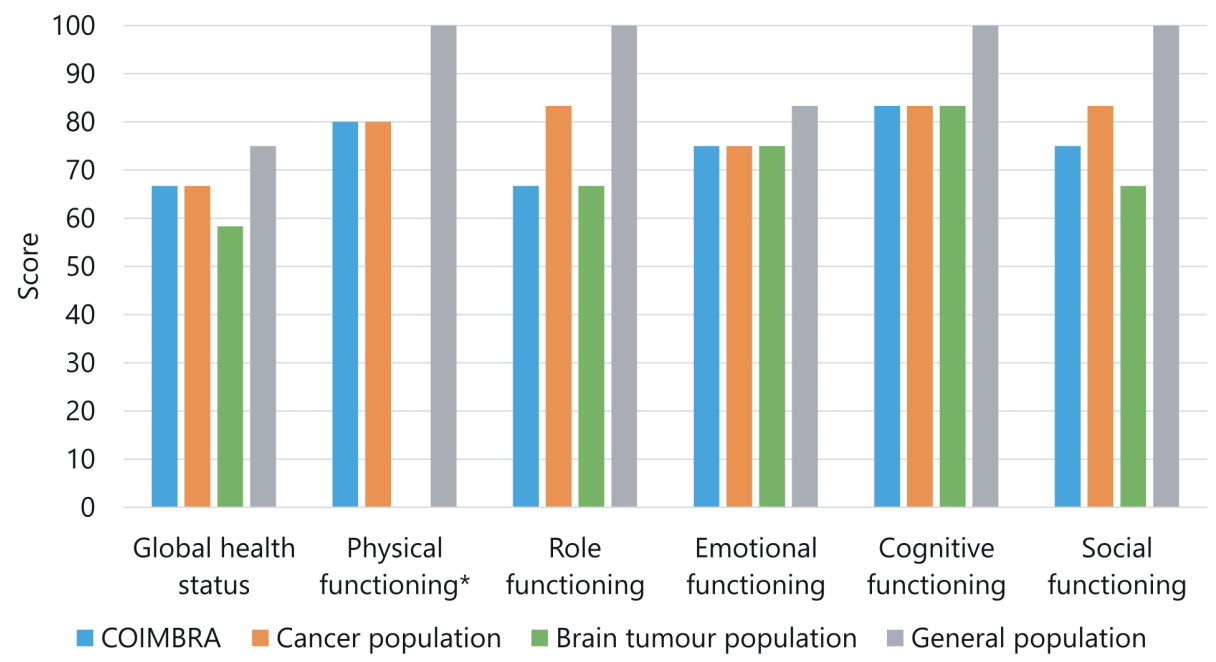

Figure 3: Comparison of QLQ-C30 sub-scores between COIMBRA, and reference values from all cancer patients, brain tumour patients and the general population [20]. Higher scores indicate better functioning.

* Reference values for physical functioning not available for brain tumour patients.

Table 2 Selected patient reported outcomes at baseline

\begin{tabular}{lllll}
\hline $\begin{array}{l}\text { Question- } \\
\text { naire }\end{array}$ & Domain/subscore & COIMBRA & $\begin{array}{l}\text { Healthy } \\
\text { population }\end{array}$ & $\begin{array}{c}\text { Cancer } \\
\text { population }\end{array}$ \\
\hline EQ-5D $^{\mathrm{b}}$ & VAS, median (IQR) & $70.0(60.0-79.8)$ & 82.0 & 68.0 \\
CFQ $^{c}$ & Total score, median (IQR) & $45(33.5-5 \mathrm{I})$ & 31.8 & 28.2 \\
HADS $^{d}$ & Anxiety score, median (IQR) & $4.5(2.3-7)$ & $5-6$ & - \\
& Depression score, median (IQR) & $4(1-7)$ & 3 & - \\
& Signs of anxiety, \% & 20.7 & - & 26.0 \\
& Signs of depression, \% & 22.4 & - & 28.2 \\
MFle & General fatigue score, median (IQR) $13(10-16)$ & $6.6-10.8$ & $11.6-12.7$ \\
& Physical fatigue score, median (IQR) & $13(10-15)$ & $6.1-11.1$ & $12.0-12.3$ \\
& Mental fatigue score, median (IQR) & $10(8-14)$ & $6.4-9.2$ & $9.1-10.1$ \\
\hline
\end{tabular}

a: Normative values taken from reference manuals and clinical studies [22-29].

b: QLQ-C30 and EQ-5D VAS scores range from 0 to 100, with a higher score indicating better outcomes

c: CFQ total score ranges from 25 to 100, with a higher score indicating more cognitive impairments $\mathrm{d}$ : HADS scores range from 0 to 21 , with a higher score indicating more anxiety or depression. A score $\geq 8$ indicates signs of anxiety or depression.

e: MFI scores range from 4 to 20 , with a higher score indicating more fatigue

$\mathrm{CFQ}=$ Cognitive Failures Questionnaire; HADS = Hospital Anxiety and Depression Scale; $\mathrm{IQR}=$ Interquartile range; MFI = Multidimensional Fatigue Inventory; VAS = Visual Analogue Scale 


\section{DISCUSSION}

In this paper, we have given an outline of the objectives and design of the COIMBRA cohort, a prospective observational cohort which facilitates conducting both randomized interventional and imaging studies. Additionally, it allows the collection of clinical and patient-reported data, providing relevant outcomes in studying new interventions.

This cohort is in line with other programs in our institute, with the adage "learning from every patient". In the department, similar studies have been set up, each with a different tumor of interest, including breast cancer, colorectal cancer and bone metastases. In each of these cohorts, the TwiCs design has been used as a basis to study new interventions, proving its utility in the inclusion of trial participants. ${ }^{18-20}$ Inclusion rates in the breast and bone metastasis cohorts are $88 \%$ and $87 \%$, higher than ours of $72.5 \%$. Questionnaire return rates are also lower in COIMBRA, which may reflect the overall higher disease burden in brain metastases patients. Another possible explanation is that digital submission of questionnaires was implemented later on in the study, which delayed its possible beneficial effects on return rates.

In addition to increased accrual rates, the TwiCs design allows conducting several randomized trials simultaneously within the same study population. ${ }^{8}$ Since the schedule of follow-up measurements is the same for each patient, not only can new interventions can be compared to standard care, but new interventions can also be compared to each other. With the caveat of interactions between new interventions, patients may also participate in multiple trials, both in the control and study arms.

Another advantage of conducting TwiCs is the process of staged informed consent. ${ }^{21}$ In the first stage, all patients eligible for COIMBRA receive information on future randomization and are asked for informed consent. In the second stage, when a new experimental intervention is studied in a TwiC, only the patients selected to undergo the intervention are informed on the trial, and are again asked for consent. This way, patients are always given information appropriate to their situation: those selected to the experimental arm are informed on the intervention, those undergoing standard care are not. In regular RCTs all eligible patients are informed on the entire study procedure, including those ultimately selected to act as controls. This means that, with a $1: 1$ randomization, half of enrolled patients are given information that does not apply to them. This can lead to higher rates of dissatisfaction and cross-over of controls, especially for treatments with promising preliminary results. ${ }^{22}$ By not informing control patients enrolled in TwiCs on their participation, this potential disappointment and confusion is prevented. 
In addition to routinely collected clinical outcomes, patient-reported measures are an important outcome in cancer treatment. ${ }^{23,24}$ Especially in a palliative setting where expected survival is limited, quality of life and absence of debilitating adverse effects of treatment can be as important as predicted survival. Therefore, the regular collection of PROs next to clinical outcomes, allows us to study the effects of new and current treatments as experienced by the patient. By gathering these data with internationally validated and implemented questionnaires, the comparability between trials and patient groups increases.

By approaching all patients referred to the radiotherapy department, not just those actually receiving radiotherapy, we collect a representative sample of brain metastasis patients. In palliative care, opting not to undergo treatment is a common phenomenon, as patients may choose to focus on quality of life above survival, or may be too frail to undergo treatment. For these patients, data on patient reported outcomes after this decision can inform both physicians and future patients on the expected outcomes of forgoing further treatment. Unfortunately, we are not able to include patients receiving palliative care or primary targeted therapy who are never referred to the radiotherapy department.

During the recruitment period, 212 patients were eligible for study inclusion. However, only 154 of those were seen by a researcher or research assistant and informed on the study. Due to national measures to prevent the spread of COVID-19, study recruitment was temporarily halted. Therefore, 32 patients with brain metastases could not be informed on the study. The other 26 patients were missed due to logistical issues. Patients can be referred to the department too quickly to schedule a research appointment, or have missed their appointment due to delays on the day of their intake at the department.

Baseline PROs can be compared to reference values from other patients, and to the healthy population. Figure $\mathbf{3}$ and Table $\mathbf{2}$ show the scores for general health status and the functioning and symptoms scores from COIMBRA and in several population groups. Compared to both patients with cancer, and to patients specifically with brain tumors, patients in COIMBRA show similar functioning scores, except regarding cognitive symptoms. The scores taken from the general population, however, show a markedly higher functioning. Due to unavailability of detailed data, we were unable to age-match the PRO results from COIMBRA to these reference populations, so no formal statistical comparisons could be made.

As seen in Table I the patients who consented to return PROs have similar clinical characteristics to those of the entire cohort, meaning these results can be more easily generalized to the entire study population. 
The possibility of performing additional imaging next to routinely made MRIs grants us the opportunity to study the effects of brain metastases and treatments on the brain. For example, interest in the effect of radiotherapy on healthy brain tissue has increased, in an effort to explain and possibly prevent cognitive decline related to morphological changes seen on MRI. ${ }^{25-27}$ Gathering more imaging data allows us to study this phenomenon in more detail, including the effects seen on MRI techniques not routinely preformed, such as diffusion tensor imaging (DTI) or functional MRI (FMRI). Studies to prevent post-RT cognitive impairments in brain metastases have already been performed. ${ }^{28}$ Our TwiCs framework, in conjunction with regular patient-reported cognitive functioning, provides a foundation to conduct similar trials, in an effort to improve quality of life after treatment. Another avenue for future research is testing the feasibility of administering radiotherapy without an immobilization mask, for which preliminary research has already been performed ${ }^{29}$. This increases patient comfort, and can pave the way for the implementation of image-guided radiotherapy techniques such as the MR-Linac. ${ }^{30}$

In conclusion, COIMBRA aims to collect prospective clinical and patient-reported outcomes, and provides an infrastructure to conduct multiple randomized trials and imaging studies in patients with brain metastases, resulting in higher trial participation and better generalizability of trial results. We expect that this will contribute to improved survival and quality of life of patients with brain metastases. First results confirm the impact of BMs on patients' cognitive symptoms, psychological well-being, daily functioning and quality of life. 


\section{REFERENCES}

I. Andrews DW, Scott CB, Sperduto PW, et al. Whole brain radiation therapy with or without stereotactic radiosurgery boost for patients with one to three brain metastases: phase III results of the RTOG 9508 randomised trial. Lancet (London, England). 2004;363(9422):1665-1672. doi:10.1016/ SOI40-6736(04)16250-8

2. Nayak L, Lee EQ, Wen PY. Epidemiology of Brain Metastases. Current Oncology Reports. 20I2;14(I):4854. doi:10.1007/s11912-011-0203-y

3. Cagney DN, Martin AM, Catalano PJ, et al. Incidence and prognosis of patients with brain metastases at diagnosis of systemic malignancy: a population-based study. Neuro-Oncology. 2017;|9(| I):|5| | - |52| . doi:10.1093/neuonc/nox077

4. Schouten LJ, Rutten J, Huveneers HAM, Twijnstra A. Incidence of brain metastases in a cohort of patients with carcinoma of the breast, colon, kidney, and lung and melanoma. Cancer. 2002;94(I0):2698-2705.

5. Nussbaum ES, Djalilian HR, Cho KH, Hall WA. Brain metastases. Histology, multiplicity, surgery, and survival. Cancer. 1996;78(8):178I-1788.

6. Lagerwaard FJ, Levendag PC, Nowak PJ, Eijkenboom WM, Hanssens PE, Schmitz PI. Identification of prognostic factors in patients with brain metastases: a review of 1292 patients. International journal of radiation oncology, biology, physics. 1999;43(4):795-803.

7. Sperduto PW, Kased N, Roberge D, et al. Summary Report on the Graded Prognostic Assessment: An Accurate and Facile Diagnosis-Specific Tool to Estimate Survival for Patients With Brain Metastases. Journal of Clinical Oncology. 2012;30(4):419-425. doi:I0.1200/JCO.2011.38.0527

8. Relton C, Torgerson D, O'Cathain A, Nicholl J. Rethinking pragmatic randomised controlled trials: introducing the "cohort multiple randomised controlled trial" design. BMJ (Clinical research ed). 2010;340:cl066. doi:I0.1136/bmj.cl066

9. van der Velden JM, Verkooijen HM, Young-Afat DA, et al. The cohort multiple randomized controlled trial design: a valid and efficient alternative to pragmatic trials? International Journal of Epidemiology. 2017;46(I):96-102. doi:10.1093/ije/dyw050

10. Scott NW, Fayers P, Aaronson NK, et al. EORTC QLQ-C30 Reference Values Manual. 2nd ed. EORTC Quality of Life Group; 2008.

II. Janssen B, Szende A. Population Norms for the EQ-5D. In: Self-Reported Population Health: An International Perspective Based on EQ-5D. Springer Netherlands; 20 14:19-30. doi: I0.1007/978-94-0077596-I_3

12. Breeman S, Cotton S, Fielding S, Jones GT. Normative data for the Hospital Anxiety and Depression Scale. Quality of Life Research. 2015;24(2):391-398. doi:I0.1007/s I I I36-014-0763-z

13. Ponds R, van Boxtel MPJ, Jolles J. De Cognitive Failure Questionnaire als maat voor subjectief cognitief functioneren. Tijdschrift voor neuropsychologie. 2006; I (2):37-45.

14. Annunziata MA, Muzzatti B, Bidoli E, et al. Hospital Anxiety and Depression Scale (HADS) accuracy in cancer patients. Supportive Care in Cancer. 2020;28(8):3921-3926. doi:10.1007/s00520-019-05244-8

15. Schwarz R, Krauss O, Hinz A. Fatigue in the General Population. Oncology Research and Treatment. 2003;26(2):140-144. doi:10.1159/000069834

16. Hinz A, Weis J, Brähler E, Härter M, Geue K, Ernst J. Fatigue in cancer patients: comparison with the general population and prognostic factors. Supportive Care in Cancer. 2020;28(9):4517-4526. doi:10.1007/s00520-019-05260-8

17. Pickard AS, Neary MP, Cella D. Estimation of minimally important differences in EQ-5D utility and VAS scores in cancer. Health and Quality of Life Outcomes. 2007;5(I):70. doi:I0.1 I86/1477-7525-5-70

18. Couwenberg AM, Burbach JPM, Berbee M, et al. Efficacy of Dose-Escalated Chemoradiation on Complete Tumor Response in Patients with Locally Advanced Rectal Cancer (RECTAL-BOOST): A Phase 2 Randomized Controlled Trial. International Journal of Radiation Oncology Biology Physics. Published online 2020. doi:10.1016/j.ijrobp.2020.06.013

19. Gal R, Monninkhof EM, Groenwold RHH, et al. The effects of exercise on the quality of life of patients with breast cancer (the UMBRELLA Fit study): study protocol for a randomized controlled trial. Trials. 2017;|8(I):504. doi:10.1/86/s|3063-017-2252-5 
20. van der Velden JM, Verkooijen HM, Seravalli E, et al. Comparing conVEntional RadioTherapy with stereotactIC body radiotherapy in patients with spinAL metastases: study protocol for an randomized controlled trial following the cohort multiple randomized controlled trial design. BMC Cancer. 2016;16(I):909. doi:10.1186/s/2885-016-2947-0

21. Vickers AJ, Young-Afat DA, Ehdaie B, Kim SY. Just-in-time consent: The ethical case for an alternative to traditional informed consent in randomized trials comparing an experimental intervention with usual care. Clinical Trials. 20 I8;15(I):3-8. doi:10.1177/1740774517746610

22. Gal R, Monninkhof EM, van Gils CH, et al. The Trials within Cohorts design faced methodological advantages and disadvantages in the exercise oncology setting. Journal of Clinical Epidemiology. 2019;113:137-146. doi:10.1016/j.jclinepi.2019.05.017

23. Nipp RD, Temel JS. Harnessing the Power of Patient-Reported Outcomes in Oncology. Clinical Cancer Research. 2018;24(8):1777-1779. doi:10.1158/1078-0432.CCR-18-0037

24. Mercieca-Bebber R, King MT, Calvert MJ, Stockler MR, Friedlander M. The importance of patientreported outcomes in clinical trials and strategies for future optimization. Patient Related Outcome Measures. 2018;Volume 9:353-367. doi:I0.2147/PROM.SI56279

25. Nagtegaal SHJ, David S, Snijders TJ, Philippens MEP, Leemans A, Verhoeff JJC. Effect of radiation therapy on cerebral cortical thickness in glioma patients: treatment-induced thinning of the healthy cortex. Neuro-Oncology Advances. 2020;2(I). doi:I0.1093/noajnI/vdaa060

26. Nagtegaal SHJ, David S, Philippens MEP, Snijders TJ, Leemans A, Verhoeff JJC. Dose-dependent volume loss in subcortical deep grey matter structures after cranial radiotherapy. Clinical and Translational Radiation Oncology. Published online November 2020. doi:I0.10 I6/j.ctro.2020.II.005

27. Gondi V, Tomé WA, Mehta MP. Why avoid the hippocampus? A comprehensive review. Radiotherapy and Oncology. 2010;97(3):370-376. doi:10.1016/j.radonc.2010.09.013

28. Gondi V, Deshmukh S, Brown PD, et al. Preservation of Neurocognitive Function (NCF) with Conformal Avoidance of the Hippocampus during Whole-Brain Radiotherapy (HA-WBRT) for Brain Metastases: Preliminary Results of Phase III Trial NRG Oncology CCOOI. International Journal of Radiation Oncology*Biology*Physics. 2018;102(5):1607. doi:10.1016/j.ijrobp.2018.08.056

29. Nagtegaal SHJ, van Lier ALHMW, den Boer AA, et al. Does an immobilization mask have added value during planning magnetic resonance imaging for stereotactic radiotherapy of brain tumours? Physics and Imaging in Radiation Oncology. 2020;13:7-13. doi:10.1016/j.phro.2020.02.003

30. Lagendijk JJW, Raaymakers BW, Raaijmakers AJE, et al. MRI/linac integration. Radiotherapy and Oncology. 2008;86(I):25-29. doi:10.1016/j.radonc.2007.10.034 


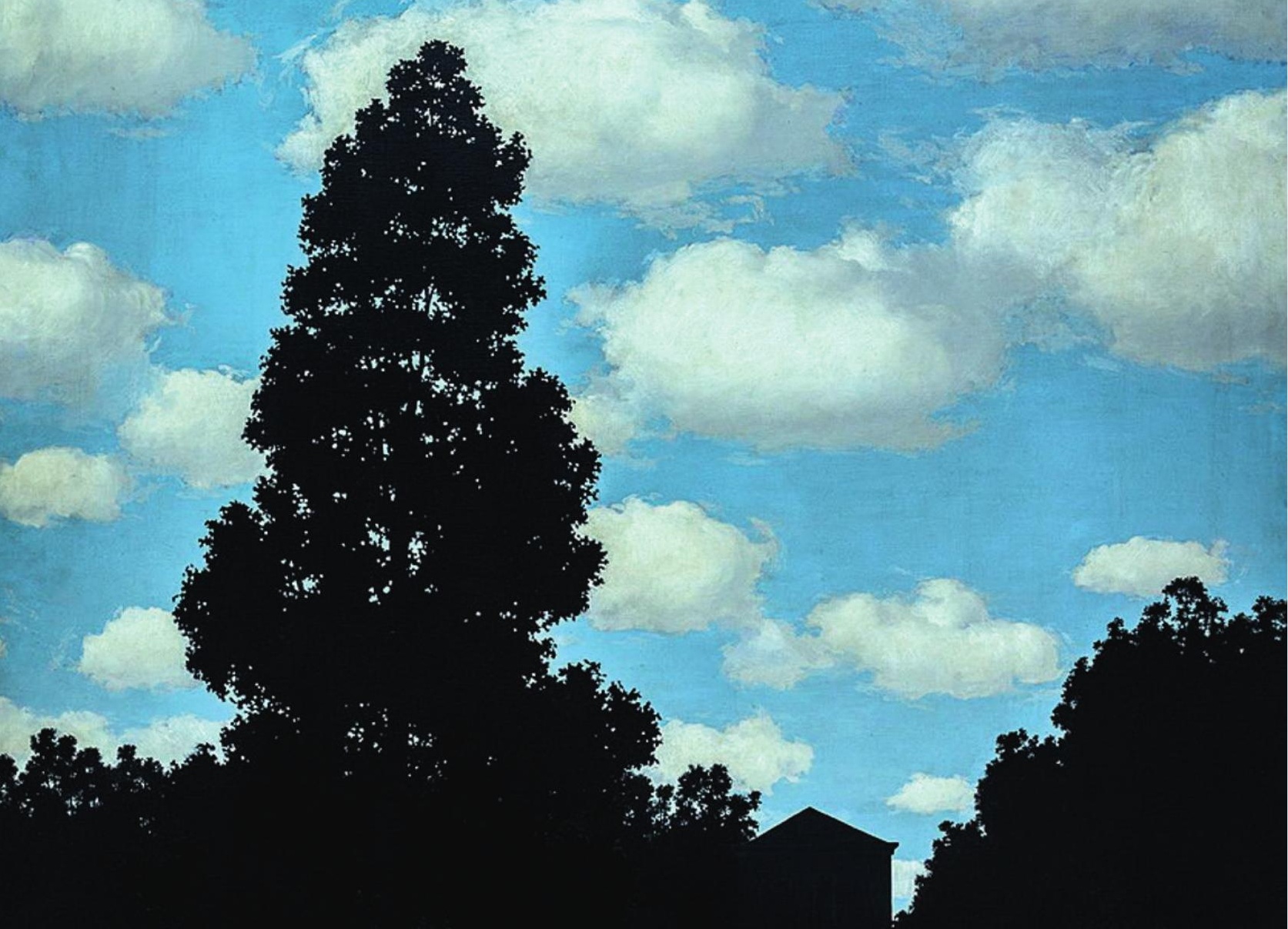

4t

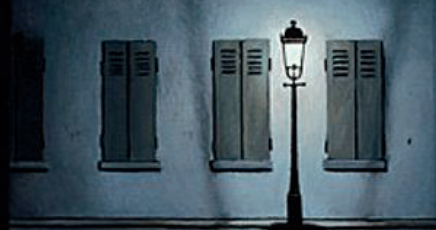




\section{Chapter 10}

General discussion 
The ultimate goal of all medical research, no matter how niche or specific, is to improve outcomes for patients. Hypotheses are generated and tested, and with these results new hypotheses are generated. Every new piece of knowledge contributes to the large and complicated medical puzzle.

The goals of the works presented in this thesis aim to contribute to: I) prevention of cognitive impairments after treatment for glioma, and 2) improvement of quality of life and survival in brain metastases patients. Obviously, no single thesis will reach these goals, but it may provide new insights and avenues for further research. Therefore, I will discuss the implications of the findings, and give suggestions for future research directions. Part I will focus on the findings of morphological changes in the brain after radiotherapy, and part II will discuss possible trials to be conducted within the framework of the COIMBRA cohort.

\section{PART I: MORPHOLOGICAL CHANGES IN THE BRAIN AFTER RADIOTHERAPY}

In part I of this thesis, I have shown that every part of the brain is susceptible to radiationinduced morphological change. On imaging performed I year after radiotherapy, the cerebral cortex shows thinning in three separate areas. Deep grey matter is also affected, with dose-dependent volume changes in six out seven subcortical structures. Finally, tissueindependent analysis has shown volumetric changes with increasing dose throughout the brain, in both white matter and grey matter. In all analyses, a consistent finding has been that the morphological changes seen are consistent with those seen after ageing by ten years. In other words, radiotherapy can be said to cause the brain to age by a decade. The clinical relevance of these studies lies in the phenomenon of radiation-induced cognitive decline. Morphological changes with increasing dose might be linked to the impairments in cognition seen after treatment. And if so, effort should be taken to prevent the change in morphology from occurring, in order to spare cognitive function. After all, higher cognitive processes are fundamental to the ability to perceive and interact with the world. Cognitive functioning is therefore a fundamental aspect of our overall quality of life (QoL).! Additionally, it affects other QoL domains such as fatigue and depression. ${ }^{2}$ And finally, cognition independently predicts survival after treatment for all grades of diffuse glioma., Although it is unlikely that improving cognition also benefits overall survival, this fact does indicate that cognition is an important outcome after treatment.

\section{Radiological findings in cognitive decline in other diseases}

This thesis shows that every part of the brain is susceptible to morphological changes after radiotherapy. A wealth of previous research demonstrated that cognitive defects occur after radiotherapy. ${ }^{5,6}$ However, evidence for the link between all three factors (cognition, radiation 
and morphology) remains scarce for all brain areas studied except the hippocampus. Luckily, we can look at studies in other diseases to strengthen the evidence of this relation, as the cognitive deficits seen in degenerative brain disease show a similar pattern to radiationinduced cognitive decline. It is also progressive and irreversible, and can range from mild symptoms to severe impairment. The only large difference is the rate in which it occurs; degenerative brain disease often has a much slower progression. Imaging has been used extensively to study the morphological changes in the brain in diseases such as mild cognitive impairment (MCl), Alzheimer's disease, and Parkinson's disease.

Loss of cortical thickness is part of the normal aging process. ${ }^{7}$ However, accelerated cortical thinning has also been observed in neurodegenerative diseases, such as Alzheimer's disease $^{8,9}$ and Parkinson's disease, ${ }^{10}$ as well as other disorders such as depression. ${ }^{11,12}$ An association between cortical thinning and cognitive impairments has also been found in $\mathrm{MCl}$ and Parkinson's, which suggests that cortical thinning could be one of the mechanisms underlying the impairments observed after radiation therapy.13,14

A link between the volumes of deep grey matter (GM) structures and cognitive outcomes has also been thoroughly examined in other brain diseases. Particularly In Alzheimer's disease, available evidence points towards a strong relation between volumes of subcortical GM structures and cognitive impairments for each of the structures we studied except for globus pallidus. ${ }^{15-17}$ Furthermore, cognitive impairments in Parkinson's disease, ${ }^{18,19}$ multiple sclerosis, ${ }^{20}$ Huntington disease, ${ }^{21}$ as well as in normal ageing, ${ }^{22}$ have been linked to the volume of at least one of the subcortical GM structures.

All these associations suggest a causal link between radiotherapy and cognitive decline, accompanied by morphological changes in the brain. However, the absence of cognitive data in our studies prohibits us from making any concrete recommendations based on our results. Furthermore, radiotherapy is not the only treatment glioma patients receive and thereby might affect cognition. Surgery and the tumour itself also have an effect on cognitive outcomes, ${ }^{23,24}$ both of which fall outside the scope of this thesis. I will therefore not discuss cognition in further detail. Instead, I will focus on ways to avoid radiation of critical healthy brain structures, in order to limit the effect of radiotherapy on cognitive outcomes, should this association truly exist.

\section{Avoiding critical structures: efforts so far}

With the knowledge and insights discussed above, experiments are conducted to prevent cognitive decline after radiotherapy by sparing critical structures. 
Cortical sparing in glioma patients has been explored in a planning study by Karunamuni et al. ${ }^{25}$ A cortical dose constraint of $28.6 \mathrm{~Gy}$ was calculated based on the probability of atrophy after treatment. Furthermore, the feasibility of applying this dose limit while maintaining adequate dose in the PTV was confirmed in a subsequent study. ${ }^{26}$ The next step is to determine the effect of cortical sparing on cognitive outcomes, which are not yet available.

Avoidance of the hippocampus during radiotherapy is much further developed. Since their discovery that hippocampal dose predicts neurocognitive outcomes of radiotherapy, the study group of Gondi et al. have designed and tested WBRT with hippocampal avoidance (HA-WBRT), see Figure I. ${ }^{27}$

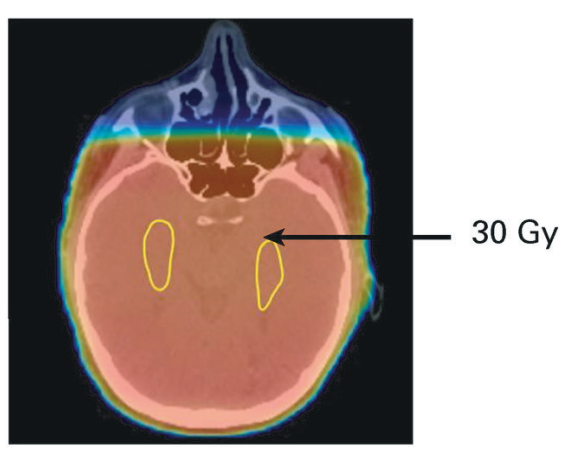

WBRT

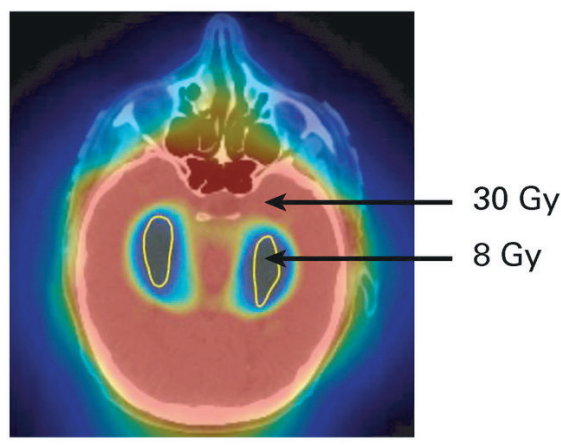

HA-WBRT

Figure I Comparison of dose distributions of WBRT with and without hippocampal avoidance (HA). Adapted from Gondi et al..$^{28}$

In this technique, the hippocampus is manually contoured on TI-weighted MRI and expanded by $5 \mathrm{~mm}$ to arrive at the hippocampal avoidance region. Intensity-modulated radiotherapy (IMRT) is used to deliver $30 \mathrm{~Gy}$ in 10 fractions to the entire brain parenchyma, while limiting the dose to $100 \%$ of the hippocampus to 9 Gy, and the maximal hippocampal dose to 16 Gy. In a phase II trial, a significant effect was found for the HVLT-R delayed recall test, with a decline from baseline of $7.0 \%$ after 4 months in the HA-WBRT group, compared to $30 \%$ in historical controls. ${ }^{29}$ This was followed by a phase III trial, in which 518 patients were randomly assigned to undergo HA-WBRT in combination with memantine, or regular WBRT with memantine. ${ }^{28}$ Similar outcomes were seen in overall survival, progression free survival and toxicity, but lower rates of memory, learning, and executive function impairments were seen in the arm in which the hippocampus was avoided. 
In contrast to HA-WBRT for established brain metastases, prophylactic cranial irradiation with hippocampal avoidance (HA-PCl) gives more divisive results. In two recent trials with small-cell lung carcinoma patients, in which $\mathrm{PCl}$ and $\mathrm{HA}-\mathrm{PCl}$ were compared, no difference in neurocognitive outcomes were seen. . $^{30,31}$

Irradiation of brain structures other than hippocampus, avoidance of which has not yet been investigated, may be involved in cognitive decline. This is acknowledged by Belderbos et al, who mention that other structures involved in cognitive processes, like amygdala, were not spared. ${ }^{30}$ As normal brain function is dependent on the connection of multiple brain structures, ${ }^{32}$ the effect of radiation on a single structure may be too narrow a focus. Furthermore, a study comparing $\mathrm{HA}-\mathrm{PCl}$ to $\mathrm{PCl}$ found significantly higher total brain doses when avoiding the hippocampus. ${ }^{33}$ This was reflected in both a higher $\mathrm{D}_{\text {max }}$, and V25 Gy and $\mathrm{V} 26 \mathrm{~Gy}$ in patients receiving $\mathrm{HA}-\mathrm{PCl}$, in addition to increased leukoencephalopathy after treatment.

This is a recurring problem when focussing on separate brain areas; sparing of one structure means other structures still receive (increased) dose. That is why I propose a different way to think about avoiding healthy tissues: considering the entire brain as organ at risk.

\section{The entire brain as organ at risk?}

In most imaging studies performed to examine the effect of radiotherapy on the healthy brain, only a specific part of the brain is studied. However, a link between local dose and volume change has now been shown to exist for the entire brain, so a reconsideration of the optimal way to preserve cognition after radiotherapy is called for.

Currently, a selected number of anatomical structures are considered "organs at risk". This distinction is based on data on susceptibility to dose, and is reserved for structures with clearly described functions. For example, the optic chiasm, retina, and brain stem each have well described, critical functions, and for each a dose threshold has been established based on decade-old data. ${ }^{34}$ As the contouring of these structures is still largely based on CT, a switch to using MRI to define organs at risk is called for.

As mentioned above, the hippocampus is considered by some to be an additional OAR. However, a future scenario can be imagined in which the entire brain is considered an organ at risk. Currently, the only used dose limit for the brain is a maximum of $60 \mathrm{~Gy}$, with no regard for radiosensitive areas or local brain function. ${ }^{35}$ As I have shown, all brain tissue types are susceptible to radiation-induced morphological changes. If a connection is found between delivered dose and cognitive impairments in some or all of these regions, a new way of performing cranial radiotherapy needs to be created. Before this can happen, two 
things need to be done. First, a detailed map of the brain needs to be made incorporating local susceptibility to dose-related adverse effects. Second, novel radiotherapy techniques need to be implemented which allow avoidance of critical regions while maintaining adequate tumour coverage.

\section{Susceptibility maps: a way to describe critical areas to avoid}

As with any scientific findings, our results give directions for new research avenues to explore. The local volume or thickness of brain tissue changes after receiving radiation dose, but how does this influence cognition, survival or quality of life after treatment? In order to answer these questions, more fine-grained data is needed on clinical outcomes after treatment, especially neurocognitive outcomes. A large, prospective cohort of patients with brain tumours, both primary and metastatic, needs to be created, in which patients undergo high-quality imaging, comprehensive neurocognitive assessments, and collection of patient-reported outcomes. This cohort will provide the foundation on which a new way to deliver radiotherapy to the brain will be based, by providing the data needed for the creation of brain susceptibility maps.

As we did in chapter 5, the analysis to create these maps needs to be done on the voxellevel. For each voxel, be it located in white matter, cerebral cortex or a subcortical nucleus, the effect of radiation to the clinical outcome of interest should be correlated. This will uncover any regional susceptibility to adverse effects caused by radiation, and can lead to identification of new areas to avoid, i.e. cerebral "no-fly zones". Furthermore, we can use knowledge from the field of connectomics, which studies the functional connections between brain regions, to improve these maps. It is possible that it is not necessarily the dose to distinct regions that leads to cognitive decline, but rather the cumulative dose to areas part of neurocognitive connectomes.

Given a large enough dataset, differences in susceptibility between patient groups (like men vs women, molecular tumour type, or age group) can be explored, in order to provide patient-specific maps.

To take it a step further, machine learning can be implemented to create optimal dose plans. Currently, planning software is given certain parameters (like "Make sure that at least 95\% of the tumour receives the prescribed dose" and "limit total dose to brain stem"), and an algorithm can design and tests thousands of iterations, until the most optimal plan is created. With knowledge on dose-susceptibility on a voxel-level, a machine learning dose-response algorithm can be trained, which predicts the expected outcomes (both in survival and toxicity) of each dose plan. ${ }^{36}$ These outcomes can be used to select the optimal plan, and to inform the patient on the expected outcome. To achieve this, a lot of computational power 
is needed. Luckily, the development and availability of systems capable of such complicated calculation is steadily increasing.

\section{Potential of new treatment delivery techniques}

Optimal treatment planning is not the only aspect that can be improved. Delivery of radiation is also a field in which progress has been made. Two of those, proton therapy and online MR-guided radiotherapy will be discussed below.

IMPT, or intensity-modulated proton therapy, has been proposed for the use in glioma before. ${ }^{37}$ In contrast to photon therapy, in which the dose is absorbed by all tissues between the points where the beam enters and exits the body, protons slow down after penetrating the tissue, with increasing release of energy as they decelerate just before they reach a complete stop (known as the Bragg peak). This phenomenon, together with the ability to direct individual beams and control their depth, means that less dose is absorbed by healthy brain tissue, while maintaining adequate tumour coverage (Figure 2).

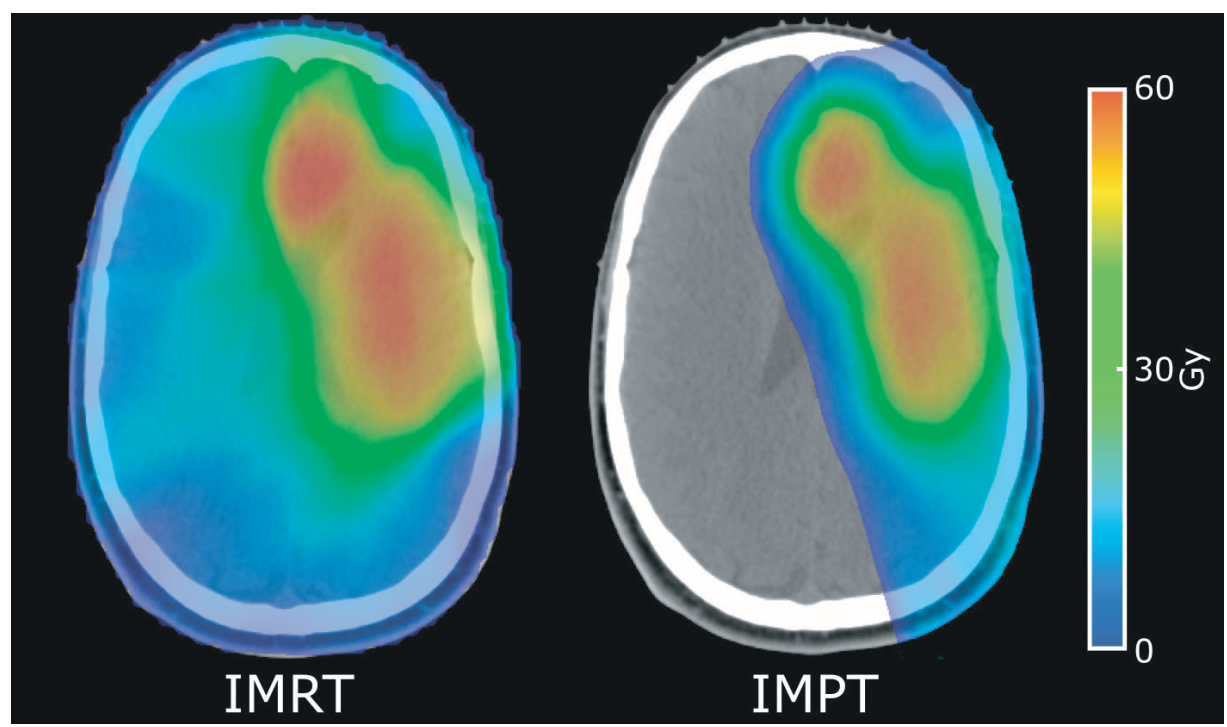

Figure 2 Example of dose-distribution with intensity modulated photon radiotherapy (IMRT), and proton therapy (IMPT). Adapted from Grosshans et al. ${ }^{37}$

MRI has been used for tumour delineation and OAR definition since its entry into clinical practice due to its superior soft-tissue contrast. A recently developed method, called online MR-guided radiotherapy, uses MR-images made before each delivered fraction to adapt to changes in daily anatomy. While this technique was largely devised to account for tumours in organs with considerable inter- and intrafraction motion (such as the abdominal 
organs), a potential benefit exists for intracranial lesions. Changes in tumour size between fractions, or between the imaging used for planning and delivery of the first fraction could be accounted for with online MR-guided adaptation. ${ }^{38,39}$ This might also allow for omission of the immobilization mask that is currently used to maintain the same position for each radiotherapy fraction. Making an MR-image to check the patient position prior to each fraction and adjusting the plans where necessary, or even real-time adjustments during the dose delivery, might mean that the mask is no longer needed. To achieve online MRguided radiotherapy, the MR-Linac was created, with combines a linear accelerator with a I.5T MRI. ${ }^{40}$

To take it a step further, imaging performed on the MR-Linac might be used in conjunction with a pre-plan made from clinical (pre-operative) scans. This means we can forgo additional planning imaging before the start of radiotherapy, and instead use the MRl's that have already been made for diagnosis or surgical navigation. To be able to do this, an additional innovation has recently been achieved, the pseudo-CT. ${ }^{4 \mid}$ While MRI is useful for tumour and OAR delineation, a CT is routinely performed to gather information on electron density needed for accurate dose calculation. As CTs are not always present for each patient, a method was created to automatically generate an image resembling a CT scan from MR images. Using a pseudo-CT instead of an actual CT, which means having an MR-only workflow, has the added benefit of reducing the error of MRI-CT registration. ${ }^{41}$

A potential scenario, which is especially desirable in case of single-fraction radiotherapy, is a "one-stop-shop" for RT. A pre-plan is made from existing MR imaging, and the necessary dose calculations are made from a generated pseudo-CT. Then, on the day of treatment, the MR-Linac is used to check the conformity of the pre-plan to the current size and location of the tumour and OARs. Adjustments to the plan are made where needed, and the dose is delivered. Next to the potential benefit of avoiding healthy brain tissue due to smaller margins, this also is a more patient-friendly and efficient way to treat brain tumours.

\section{PART II: SURVIVAL AND PATIENT-REPORTED OUTCOMES IN BRAIN METASTASES}

In part II of this thesis describes several studies into the effects of clinical factors on survival of patients with brain metastasis, and contains an overview of the aims and design of the Cohort for patient-reported Outcomes, Imaging and trial inclusion in Metastatic BRAin disease (COIMBRA), a prospective cohort from which trials within cohorts (TwiCs) can be performed. Here, I will discuss how prognostic information can used, and will discuss several potential new studies that can be performed using the data and infrastructure COIMBRA provides us. 


\section{Prognostic tools in oncology: does the patient benefit?}

Treatment of cancer is constantly improving. Countless new interventions are continuously being tested, approved, and implemented. With these, we hope to improve tumour control and survival preferably with good quality of life. With this multitude of treatment options, it is easy to forget that a crucial part of palliative care is to know when to stop treating the disease, and to focus on the patient's quality of life in the remaining months or years. This requires physicians to have an honest conversation with their patients, to discuss the expected effects (and side-effects) of treatment, and to give an estimation of the survival benefit it gives. In addition to being an ethical requirement, this is a legal imperative for many health professionals. For example, the Dutch Medical Treatment Contracts Act (Wet Geneeskundige Behandelovereenkomst, WGBO), states that physicians should give clear information on a patient's health status, and any proposed treatments or medical tests. ${ }^{42}$

In order to do this, however, physicians need accurate data, updated to the latest knowledge on treatments and prognostics. Therefore, we conducted several studies to improve the scientific basis for making shared decisions in the care of brain metastases.

We have studied the accuracy and applicability of the disease-specific graded prognostic assessment (DS-GPA) in a cohort of brain metastases patients treated with stereotactic radiosurgery (SRS). The DS-GPA is a widely used tool to estimate survival in patients with brain metastases from the five most common primary tumour types: lung carcinoma, breast cancer, melanoma, renal cell cancer, and gastro-intestinal cancer. ${ }^{43}$ Based on several clinical and molecular factors at baseline, it gives an estimation of the median survival after treatment. The fact that this estimation is indeed a median is an important finding in our study, as it is not a point prediction. Looking at Figure 3, we can see that the difference between the actual survival and the predicted median can be quite large, and exceed 40 months. This means that, while on a group level the survival predicted by the DS-GPA may be accurate, on a patient level this is not necessarily the case. This variety in survival of brain metastases patients has been acknowledged by the creators of the DS-GPA. ${ }^{43}$ 
3 or fewer brain metastases

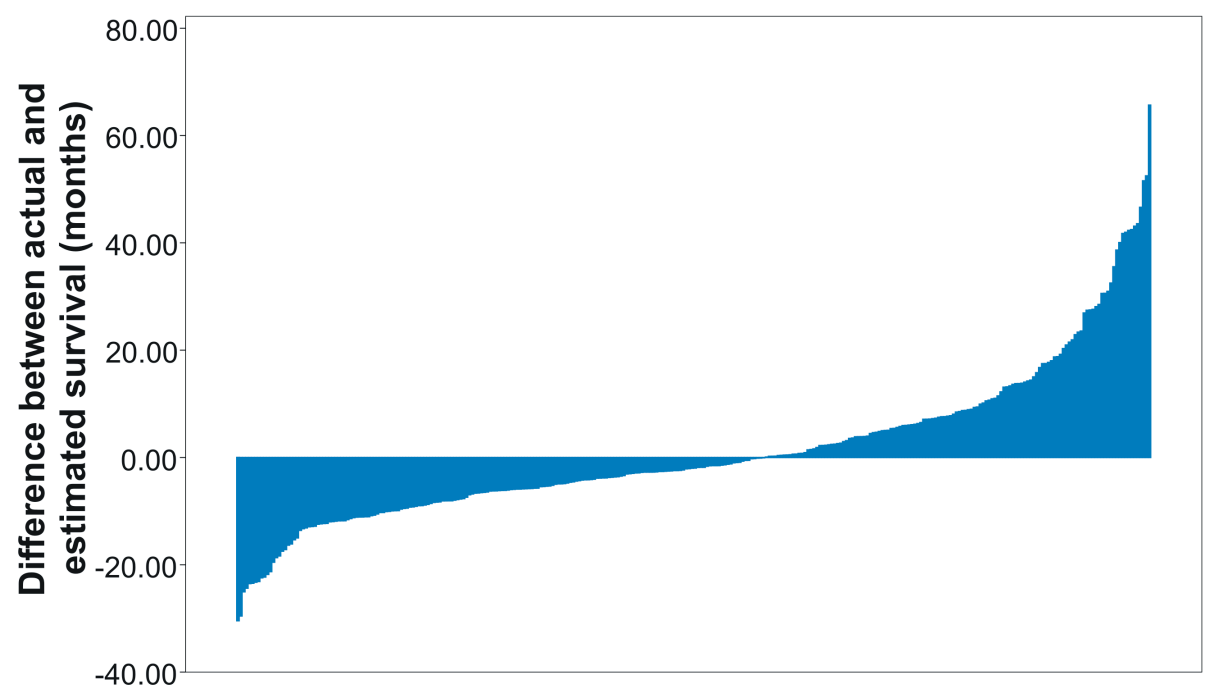

Figure 3 Waterfall plot showing the difference between the median survival estimated by the DS-GPA, and the actual survival in patients treated with SRS for I-3 brain metastases. Adapted from Nagtegaal et al. ${ }^{44}$

In structured interviews with patients diagnosed with high-grade glioma, the communication surrounding the diagnosis and its prognosis were explored. ${ }^{45}$ After the shock of hearing the diagnosis, the information on expected survival was hard to process for some patients. Some participants had a difficulty in understanding the prognostic implications, and were not always able to recollect every detail discussed. Assuming a similar feeling exists in patients with brain metastases, explaining the results of the DS-GPA model as a median survival may not always be appropriate. The concept of "estimated median survival" may be hard enough to understand outside this difficult context, let alone when it concerns your own survival. Furthermore, some patients did not want to receive detailed information at all, but would have rather heard more general information on life expectancy (i.e., limited to "months" or "years"). Therefore, as with treatment, a patient-tailored approach is called for when discussing prognosis. The first step should be gauging the extent to which a patient wants to receive information on the expected outcomes of the disease and its treatment. After that, detailed information (with the necessary caveats) can be given, but only if a patient and their caregivers wish to receive it. This latter point is also included in the abovementioned WGBO, stating that patients can choose not to receive information on their health. ${ }^{42} \mathrm{As}$ the results from these structured interviews may not be fully applicable to brain metastases patients, they could be repeated within the COIMBRA cohort. Additionally, a trial could be set up in which patients can choose to receive detailed prognostic information (such 
as the results of the DS-GPA), using the anxiety and depression scores from the HADS questionnaire to assess the impact on QoL.

\section{Patient-reported outcomes: opportunities and challenges}

In any medical setting, patient-reported outcomes are an important measure to assess the effect of treatment. When the estimated survival is limited, the health-related quality of life is a very relevant outcome to patients. Although the survival of patients with brain metastases has improved substantially due to new radiotherapy techniques and improved immunotherapeutic and targeted options, the majority of patients can still be considered to be in a palliative or end-of-life setting. ${ }^{47,48}$ Therefore, we have implemented the collection of extensive patient-reported outcomes in COIMBRA, a longitudinal cohort of patients with brain metastases. At fixed timepoints after inclusion, data are gathered on overall quality of life and its main components: cognition, fatigue, mental health and physical activity. Below, I will discuss challenges in analysing and interpreting these results, and give recommendations on how to account for them.

Firstly, the radiotherapy department is just one of the medical specialties involved in the treatment of patients. Brain metastases are mostly treated by a team of radiation oncologists, medical oncologists and lung oncologists, neurosurgeons and neurologists. For those with a known primary tumour, this list extends even further. Each of the treatments given by these specialists affects quality of life, next to the countless other factors that contribute to a patient's wellbeing. This means it will be challenging to assess the effect of a single factor (such as SRS vs WBRT) in an observational setting where patients undergo a variety of treatments and therapies at different time points. A way to overcome this would be to gather a large number of patients, so you could either correct for these factors in statistical modelling, or create subgroups of adequate size for meaningful comparisons. Another obvious solution is to compare treatments in a randomized trial, which the TwiCs designs allows us to do. When randomization is successful, any factors that could influence the results are independent of the likelihood of allocation to the treatment and control arms.

Secondly, we have seen that the return rates in our cohort (with preliminary rates of $70.9 \%$ at baseline and $39.0 \%$ at 6 months) are lower than those of similar cohorts in our department. ${ }^{49}$ An explanation might be that participation rates are lower when disease burden is higher, because patients might not feel well enough to complete the questionnaires. Additionally, cognitive impairments as a consequence of the brain lesion may also limit the ability to understand the questions. Treatment can be curative in other cancer cases, in contrast to the palliative setting that most brain metastasis patients are in. As more patients are enrolled in COIMBRA, the patient characteristics of those not 
returning questionnaires (in addition to those opting out of collection of PROs) should be analysed to assess the level of bias this missing data creates. If higher symptom burden, such as increased fatigue or cognitive impairment, is a reason that participants do not return the questionnaires, the measured QoL within COIMBRA is higher than the normal brain metastases population and are therefore not representative. Furthermore, when conducting TwiCs with QoL as an endpoint, failing to capture low QoL scores may lead to a bias in the effect of the studied intervention. Discovering which factors are associated with poorer return rates might help correct for this bias.

The above should be done in parallel with efforts to increase return rates. A digital platform in which questionnaires can be completed has already been implemented, the effect of which will reveal itself the coming year. Another method to increase participation could be reporting the results of the questionnaires back to the patients, showing to what extent QoL changes during the course of treatment. However, current return rates may already be maximal, as drop-out in PRO participation has been high in previous trials with BM patients. ${ }^{50}$

Finally, the phenomenon of response shift is a recurring challenge when comparing PROs of cancer patients - including those with brain metastases - to the general healthy population. ${ }^{50,51}$ Generally, response shift can occur in health-related quality outcomes due to a changing perception of the concept of health and its facets throughout the course of the disease. After being diagnosed with cancer, patients may recalibrate their standards of measuring QoL, reprioritize certain outcomes, or reconceptualise the construct of health. Simply put, one's perception of what constitutes good or poor health may change after receiving a cancer diagnosis. This complicates any comparison between patient groups and healthy populations. It may be expected that this affects mostly broad questions within the questionnaires (i.e. questions 29 and 30 from the QLQ-C30, which ask patients to rate overall health and QoL on a seven-point scale) $)^{52}$; objective questions on e.g. hair-loss or seizures are likely less susceptible to response shifts. ${ }^{53}$ Therefore, while it may be tempting to focus on the overall self-reported quality of life, composite scores based on more objective reporting of symptoms should definitely be taken into account. Specially created symptom questionnaires such as the MD Anderson Symptom Inventory or the Utrecht Symptom Diary may be additional resources to achieve this. ${ }^{54,55}$

Furthermore, comparisons between patient groups and the healthy population, as well as comparisons between time points within the patient group, should always be done with response shift in mind, so as not to make misguided conclusions on the quality of life of brain metastases patients. 


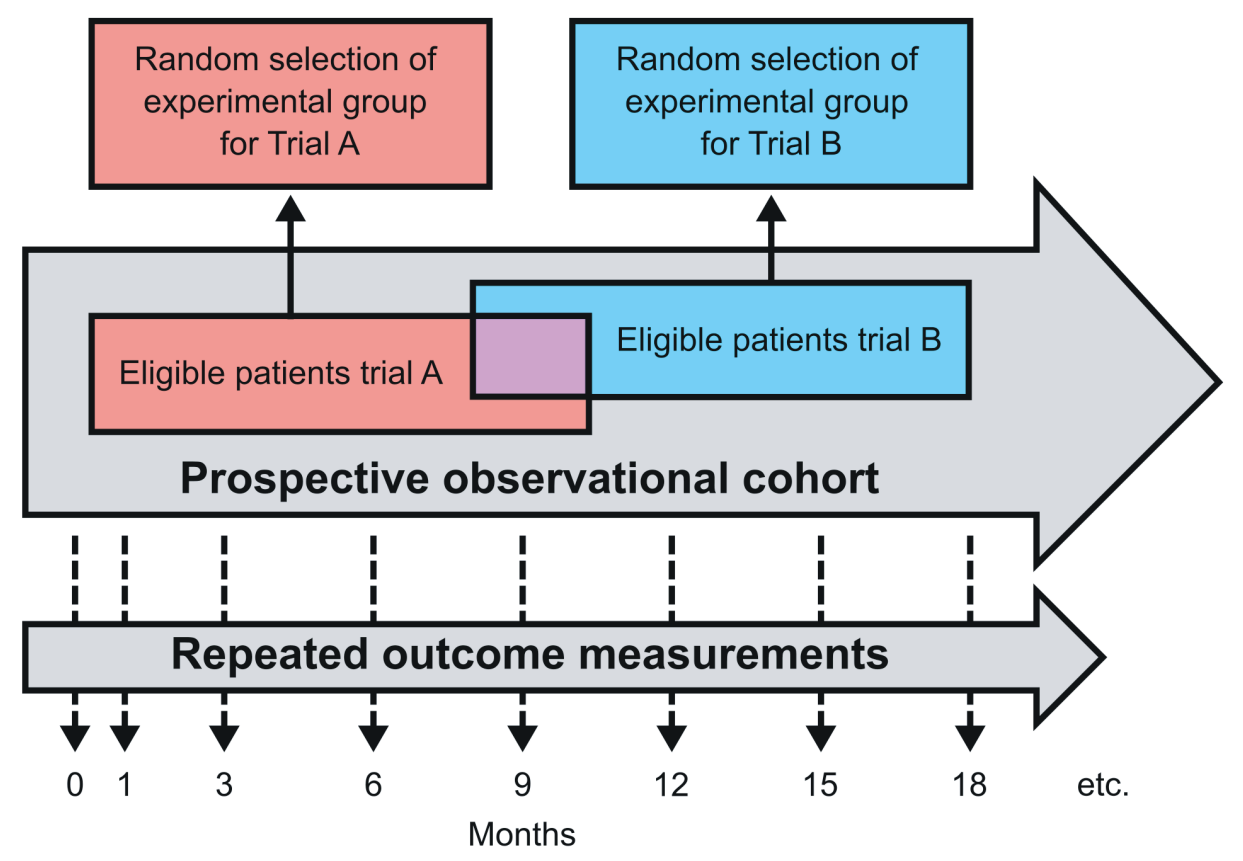

Figure 4 Graphic representation of the COIMBRA cohort. After inclusion, PROs are collected at fixed time points. Multiple randomized trials (red and blue) can be conducted simultaneously. For each randomized trial a sub-cohort of eligible patient is identified. Patients from the sub-cohort are randomly selected to undergo the intervention, and their outcomes are then compared to the other patients in the sub-cohort who undergo standard care. Adapted from Relton et al. ${ }^{56}$

\section{Using the Trials within Cohorts design for future research}

The TwiCs design provides an infrastructure to conduct several randomized trials at once, with the potential of increased rates of accrual and lower drop-out (Figure 4$)^{56}$. The opportunities for new trials are of course limitless, so below I will discuss several potential avenues of research based on the findings in the thesis and the discussion above.

Firstly, trials on avoidance of cerebral areas susceptible to radiation-induced might result in new recommendations for future radiotherapy guidelines. Like the trial of hippocampal avoidance described above, we might study the effect of avoiding more or all subcortical nuclei or cortical regions in patients with brain metastases. Before this can be done, one challenge needs to be overcome: there is currently no way to measure cognitive outcomes in the control group of a TwiC run in COIMBRA without informing them on trial participation. This might be solved by simply not strictly adhering to the TwiC process, or by temporarily adding routine neurocognitive testing to COIMBRA as an extra opt-in study procedure. 
Secondly, and perhaps in parallel with the point above, we could assess the utility of the new techniques IMPT and IGRT. As the UMC Utrecht currently does not apply proton therapy, but is at the forefront of MR-guided radiotherapy, the latter technique is an obvious starting point. As mentioned in part I, creating an MR image prior to each fraction might improve the accuracy of the delivered treatment. As current treatment margins are in place to correct for any technical uncertainty, using a more precise technique might mean these margins can be reduced. Furthermore, the one-stop-shop solution mentioned in part I may also be tested within COIMBRA. Data on PROs are especially relevant in this context, even more so when adding questionnaires specifically asking about treatment comfort.

This final point, using PROs to explore not only the efficacy of administered treatments but also the satisfaction with the whole clinical experience, would be my third suggestion. Since last year OSIRIS (One Stop IRradiation for Intracranial Sites) has been implemented in the UMC Utrecht for patients referred for SRS of brain metastases. Where in the past the time between intake and treatment could be several weeks, with OSIRIS patients are treated within seven days. This also allows faster administration of adjuvant therapy. Not only do we expect survival and local control to improve with faster treatment, we also think this will improve QoL. When enough data is gathered, the PROs of the patient treated under OSIRIS can be compared to historical controls from the prior workflow. This will allow us to ultimately improve the care for patients with brain metastases on all fronts, providing the most optimal care for future patients.

\section{CLOSING REMARKS}

The studies presented in this thesis can inspire future research into the positive and negative effects of cranial radiotherapy on the wellbeing of patients. For primary tumours, a complete approach is necessary, in which the entire brain is studied. Only then can we give recommendations for avoiding areas crucial for cognitive functioning. Secondly, the outcomes of trials should incorporate patient-reported quality of life, as this might well be as important as lesion control and survival. Just like in clinical practice, a comprehensive view is important in clinical research. Only when healthcare providers, consider every part of the patient, from the most specific molecular markers or cortical area to their overall self-reported health, can we achieve results and give recommendations that are meaningful to future patients with brain tumours. 


\section{REFERENCES}

I. Boele FW, Zant M, Heine ECE, et al. The association between cognitive functioning and health-related quality of life in low-grade glioma patients. Neuro-Oncology Practice. 20 I4;I(2):40-46. doi:I0. I093/nop/ npu007

2. Liu R, Page M, Solheim K, Fox S, Chang SM. Quality of life in adults with brain tumors: Current knowledge and future directions. Neuro-Oncology. 2009;II(3):330-339. doi:I0.1215/I52285I7-2008-093

3. van Kessel E, Huenges Wajer IMC, Ruis C, et al. Cognitive impairments are independently associated with shorter survival in diffuse glioma patients. Journal of Neurology. Published online November 19 , 2020. doi:10.1007/s00415-020-10303-w

4. Klein M, Postma TJ, Taphoorn MJB, et al. The prognostic value of cognitive functioning in the survival of patients with high-grade glioma. Neurology. 2003;6I(I2):1796-1798. doi:10.1212/01. WNL.0000098892.33018.4C

5. Makale MT, McDonald CR, Hattangadi-Gluth JA, Kesari S. Mechanisms of radiotherapy-associated cognitive disability in patients with brain tumours. Nature Reviews Neurology. 2017;13(I):52-64. doi:10.1038/nrneurol.2016.185

6. Greene-Schloesser D, Robbins ME. Radiation-induced cognitive impairment-from bench to bedside. Neuro-Oncology. 2012;14(SUPPL.4):iv37-iv44. doi:I0.1093/neuonc/nos196

7. Salat DH. Thinning of the Cerebral Cortex in Aging. Cerebral Cortex. 2004;14(7):72I-730. doi:I0.1093/ cercor/bhh032

8. Du A-T, Schuff N, Kramer JH, et al. Different regional patterns of cortical thinning in Alzheimer's disease and frontotemporal dementia. Brain. 2007;130(4):I 159-II66. doi:10.1093/brain/awm0I6

9. Yin C, Li S, Zhao W, Feng J. Brain imaging of mild cognitive impairment and Alzheimer's disease. Neural regeneration research. 2013;8(5):435-444. doi:I0.3969/j.issn. I673-5374.2013.05.007

10. Pereira JB, Ibarretxe-Bilbao N, Marti M-J, et al. Assessment of cortical degeneration in patients with Parkinson's disease by voxel-based morphometry, cortical folding, and cortical thickness. Human Brain Mapping. 2012;33(I I):2521-2534. doi:10.1002/hbm.21378

II. Lim HK, Jung WS, Ahn KJ, et al. Regional cortical thickness and subcortical volume changes are associated with cognitive impairments in the drug-naive patients with late-onset depression. Neuropsychopharmacology. 2012;37(3):838-849. doi:10.1038/npp.2011.264

12. Peterson BS, Warner $V$, Bansal R, et al. Cortical thinning in persons at increased familial risk for major depression. Proceedings of the National Academy of Sciences. 2009;106(I5):6273-6278. doi:10.1073/ pnas.0805311106

13. Seo SW, Im K, Lee J-M, et al. Cortical thickness in single- versus multiple-domain amnestic mild cognitive impairment. Neurolmage. 2007;36(2):289-297. doi:10.1016/j.neuroimage.2007.02.042

14. Garcia-Diaz Al, Segura B, Baggio HC, et al. Cortical thinning correlates of changes in visuospatial and visuoperceptual performance in Parkinson's disease: A 4-year follow-up. Parkinsonism \& Related Disorders. 2018;46:62-68. doi:10.1016/j.parkreldis.2017.11.003

15. de Jong LW, van der Hiele K, Veer IM, et al. Strongly reduced volumes of putamen and thalamus in Alzheimer's disease: an MRI study. Brain: a journal of neurology. 2008;131(Pt 12):3277-3285. doi:10.1093/brain/awn278

16. Nie X, Sun Y, Wan S, et al. Subregional Structural Alterations in Hippocampus and Nucleus Accumbens Correlate with the Clinical Impairment in Patients with Alzheimer's Disease Clinical Spectrum: Parallel Combining Volume and Vertex-Based Approach. Frontiers in neurology. 20 17;8:399. doi:10.3389/fneur.2017.00399

17. Yi H-A, Möller C, Dieleman N, et al. Relation between subcortical grey matter atrophy and conversion from mild cognitive impairment to Alzheimer's disease. Journal of Neurology, Neurosurgery \& Psychiatry. 2016;87(4):425-432. doi:I0.1136/jnnp-2014-309105

18. Hanganu A, Bedetti C, Degroot C, et al. Mild cognitive impairment is linked with faster rate of cortical thinning in patients with Parkinson's disease longitudinally. Brain : a journal of neurology. 2014;137(Pt 4): I |20- | |29. doi: I0.1093/brain/awu036 
19. Hünerli D, Emek-Savaş DD, Çavuşoğlu B, Dönmez Çolakoğlu B, Ada E, Yener GG. Mild cognitive impairment in Parkinson's disease is associated with decreased $\mathrm{P} 300$ amplitude and reduced putamen volume. Clinical Neurophysiology. 2019;130(8):I208-1217. doi:10.1016/j.clinph.2019.04.314

20. Rojas JI, Murphy G, Sanchez F, et al. Thalamus volume change and cognitive impairment in early relapsing-remitting multiple sclerosis patients. The neuroradiology journal. 2018;31(4):350-355. doi:10.1177/1971400918781977

21. Aylward EH, Harrington DL, Mills JA, et al. Regional atrophy associated with cognitive and motor function in prodromal Huntington disease. Journal of Huntington's disease. 2013;2(4):477-489. doi:10.3233/JHD-130076

22. Hughes EJ, Bond J, Svrckova P, et al. Regional changes in thalamic shape and volume with increasing age. Neurolmage. 2012;63(3):I I34-II42. doi: I0. I016/j.neuroimage.2012.07.043

23. Satoer D, Visch-Brink E, Smits M, et al. Long-term evaluation of cognition after glioma surgery in eloquent areas. Journal of Neuro-Oncology. 2014;1 I6(I):153-160. doi:10.1007/s I 060-013-1275-3

24. Van Kessel E, Emons MAC, Wajer IH, et al. Tumor-related neurocognitive dysfunction in patients with diffuse glioma: A retrospective cohort study prior to antitumor treatment. Neuro-Oncology Practice. 2019;6(6):463-472. doi:10.1093/nop/npz008

25. Karunamuni RA, Moore KL, Seibert TM, et al. Radiation sparing of cerebral cortex in brain tumor patients using quantitative neuroimaging. Radiotherapy and oncology : journal of the European Society for Therapeutic Radiology and Oncology. 2016;118(I):29-34. doi:10.1016/j.radonc.2016.01.003

26. Exeli A-K, Kellner D, Exeli L, et al. Cerebral cortex dose sparing for glioblastoma patients: IMRT versus robust treatment planning. Radiation oncology (London, England). 2018;13(I):20. doi:10.1186/ sl3014-018-0953-x

27. Gondi V, Tolakanahalli R, Mehta MP, et al. Hippocampal-sparing whole-brain radiotherapy: a "how-to" technique using helical tomotherapy and linear accelerator-based intensity-modulated radiotherapy. International journal of radiation oncology, biology, physics. 2010;78(4):1244-1252. doi:10.1016/j. ijrobp.2010.01.039

28. Brown PD, Gondi V, Pugh S, et al. Hippocampal Avoidance During Whole-Brain Radiotherapy Plus Memantine for Patients With Brain Metastases: Phase III Trial NRG Oncology CCOOI. Journal of clinical oncology : official journal of the American Society of Clinical Oncology. Published online February 14, 2020:JCO1902767. doi:10.1200/JCO.19.02767

29. Gondi V, Pugh SL, Tome WA, et al. Preservation of memory with conformal avoidance of the hippocampal neural stem-cell compartment during whole-brain radiotherapy for brain metastases (RTOG 0933): A phase II multi-institutional trial. Journal of Clinical Oncology. 2014;32(34):38I0-38I6. doi:I0.1200/JCO.20I4.57.2909

30. Belderbos J, de Ruysscher D, de Jaeger K, et al. Phase III Randomized Trial of Prophylactic Cranial Irradiation With or Without Hippocampus Avoidance in SCLC. SSRN Electronic Journal. Published online 2020. doi:10.2139/ssrn.3708322

31. Vees H, Caparrotti F, Eboulet El, et al. Impact of Early Prophylactic Cranial Irradiation With Hippocampal Avoidance on Neurocognitive Function in Patients With Limited Disease Small Cell Lung Cancer. A Multicenter Phase 2 Trial (SAKK 15/I2). International Journal of Radiation Oncology*Biology*Physics. 2020;107(2):279-287. doi:I0.1016/j.jirobp.2020.02.029

32. Bullmore E, Sporns O. Complex brain networks: Graph theoretical analysis of structural and functional systems. Nature Reviews Neuroscience. 2009;10(3):186-198. doi:10.1038/nrn2575

33. Mayinger M, Kraft J, Lohaus $\mathrm{N}$, et al. Leukoencephalopathy after prophylactic whole-brain irradiation with or without hippocampal sparing: a longitudinal magnetic resonance imaging analysis. European Journal of Cancer. 2020;124:194-203. doi:10.1016/j.ejca.2019.11.008

34. Emami B, Lyman J, Brown A, et al. Tolerance of normal tissue to therapeutic irradiation. International Journal of Radiation Oncology, Biology, Physics. 1991;21 (I):I09-122. doi:10.1016/0360-3016(91)9017I-Y

35. Walker MD, Alexander E, Hunt WE, et al. Evaluation of BCNU and/or radiotherapy in the treatment of anaplastic gliomas. Journal of Neurosurgery. 1978;49(3):333-343. doi:I0.317//jns. 1978.49.3.0333

36. Boldrini L, Bibault J-E, Masciocchi C, Shen Y, Bittner M-I. Deep Learning: A Review for the Radiation Oncologist. Frontiers in Oncology. 2019;9:977. doi:10.3389/fonc.2019.00977 
37. Grosshans DR, Mohan R, Gondi V, Shih HA, Mahajan A, Brown PD. The role of image-guided intensity modulated proton therapy in glioma. Neuro-Oncology. 2017;19(suppl_2):ii30-ii37. doi:10.1093/neuonc/ nox002

38. Cao Y, Tseng C-L, Balter JM, Teng F, Parmar HA, Sahgal A. MR-guided radiation therapy: transformative technology and its role in the central nervous system. Neuro-Oncology. 2017;19(suppl_2):iil6-ii29. doi:I0.1093/neuonc/nox006

39. Corradini S, Alongi F, Andratschke N, et al. MR-guidance in clinical reality: current treatment challenges and future perspectives. Radiation Oncology. 2019;14(I):92. doi:10.1186/s13014-019-1308-y

40. Lagendijk JJW, Raaymakers BW, Raaijmakers AJE, et al. MRI/linac integration. Radiotherapy and Oncology. 2008;86(1):25-29. doi:10.1016/j.radonc.2007.10.034

41. Dinkla AM, Wolterink JM, Maspero M, et al. MR-Only Brain Radiation Therapy: Dosimetric Evaluation of Synthetic CTs Generated by a Dilated Convolutional Neural Network. International Journal of Radiation Oncology*Biology*Physics. 2018;102(4):80I-8I2. doi:I0.1016/j.jijrobp.2018.05.058

42. Wet Geneeskundige Behandelovereenkomst. Published 2020. https://wetten.overheid.nl/ BWBR0005290/2021-01-01

43. Sperduto PW, Kased N, Roberge D, et al. Summary Report on the Graded Prognostic Assessment: An Accurate and Facile Diagnosis-Specific Tool to Estimate Survival for Patients With Brain Metastases. Journal of Clinical Oncology. 2012;30(4):419-425. doi:10.1200/JCO.2011.38.0527

44. Nagtegaal SHJ, Claes A, Suijkerbuijk KPM, Schramel FMNH, Snijders TJ, Verhoeff JJC. Comparing survival predicted by the diagnosis-specific Graded Prognostic Assessment (DS-GPA) to actual survival in patients with $1-10$ brain metastases treated with stereotactic radiosurgery. Radiotherapy and Oncology. 2019;138:173-179. doi:10.1016/j.radonc.2019.06.033

45. Lobb EA, Halkett GKB, Nowak AK. Patient and caregiver perceptions of communication of prognosis in high grade glioma. Journal of Neuro-Oncology. 20 II; I04(I):315-322. doi: I0. I007/s I 1060-0 I0-0495-z

46. Sperduto PW. GPA Index. Published 2018. www.brainmetgpa.com

47. Nayak L, Lee EQ, Wen PY. Epidemiology of Brain Metastases. Current Oncology Reports. 2012;14(I):4854. doi: I0.1007/s| |9|2-01I-0203-y

48. Schouten LJ, Rutten J, Huveneers HAM, Twijnstra A. Incidence of brain metastases in a cohort of patients with carcinoma of the breast, colon, kidney, and lung and melanoma. Cancer. 2002;94(I0):2698-2705.

49. Young-Afat DA, van Gils CH, van den Bongard HJGD, Verkooijen HM, UMBRELLA Study Group. The Utrecht cohort for Multiple BREast cancer intervention studies and Long-term evaLuAtion (UMBRELLA): objectives, design, and baseline results. Breast cancer research and treatment. 2017;164(2):445-450. doi:10.1007/s10549-017-4242-4

50. Lin NU, Wefel JS, Lee EQ, et al. Challenges relating to solid tumour brain metastases in clinical trials, part 2: neurocognitive, neurological, and quality-of-life outcomes. A report from the RANO group. The Lancet Oncology. 20I3;14(I0):e407-e416. doi:I0.1016/SI470-2045(I3)70308-5

51. Schwartz CE, Andresen EM, Nosek MA, Krahn GL. Response Shift Theory: Important Implications for Measuring Quality of Life in People With Disability. Archives of Physical Medicine and Rehabilitation. 2007;88(4):529-536. doi:10.1016/j.apmr.2006.12.032

52. Groenvold M, Klee MC, Sprangers MAG, Aaronson NK. Validation of the EORTC QLQ-C30 quality of life questionnaire through combined qualitative and quantitative assessment of patient-observer agreement. Journal of Clinical Epidemiology. 1997;50(4):44I-450. doi:I0. I 1 I6/S0895-4356(96)00428-3

53. Sprangers MAG, Schwartz CE. Integrating response shift into health-related quality of life research: a theoretical model. Social Science \& Medicine. 1999;48(II):1507-1515. doi:10.1016/S02779536(99)00045-3

54. Cleeland CS, Mendoza TR, Wang XS, et al. Assessing symptom distress in cancer patients: the M.D. Anderson Symptom Inventory. Cancer. 2000;89(7):1634-1646. doi:10.1002/10970I42(2000I00I)89:7<1634::aid-cncr29>3.0.co;2-v

55. IJzerman-Korevaar M, Snijders TJ, Teunissen SCCM, de Graeff A, De Vos FYF. Symptom Monitoring in Glioma Patients: Development of the Edmonton Symptom Assessment System Glioma Module. The Journal of neuroscience nursing: journal of the American Association of Neuroscience Nurses. 2018;50(6):38I-387. doi:I0.1097/JNN.0000000000000400 
Chapter 10

56. Relton C, Torgerson D, O'Cathain A, Nicholl J. Rethinking pragmatic randomised controlled trials: introducing the "cohort multiple randomised controlled trial" design. BMJ (Clinical research ed). 2010;340:cl066. doi:10.1136/bmj.cl066 


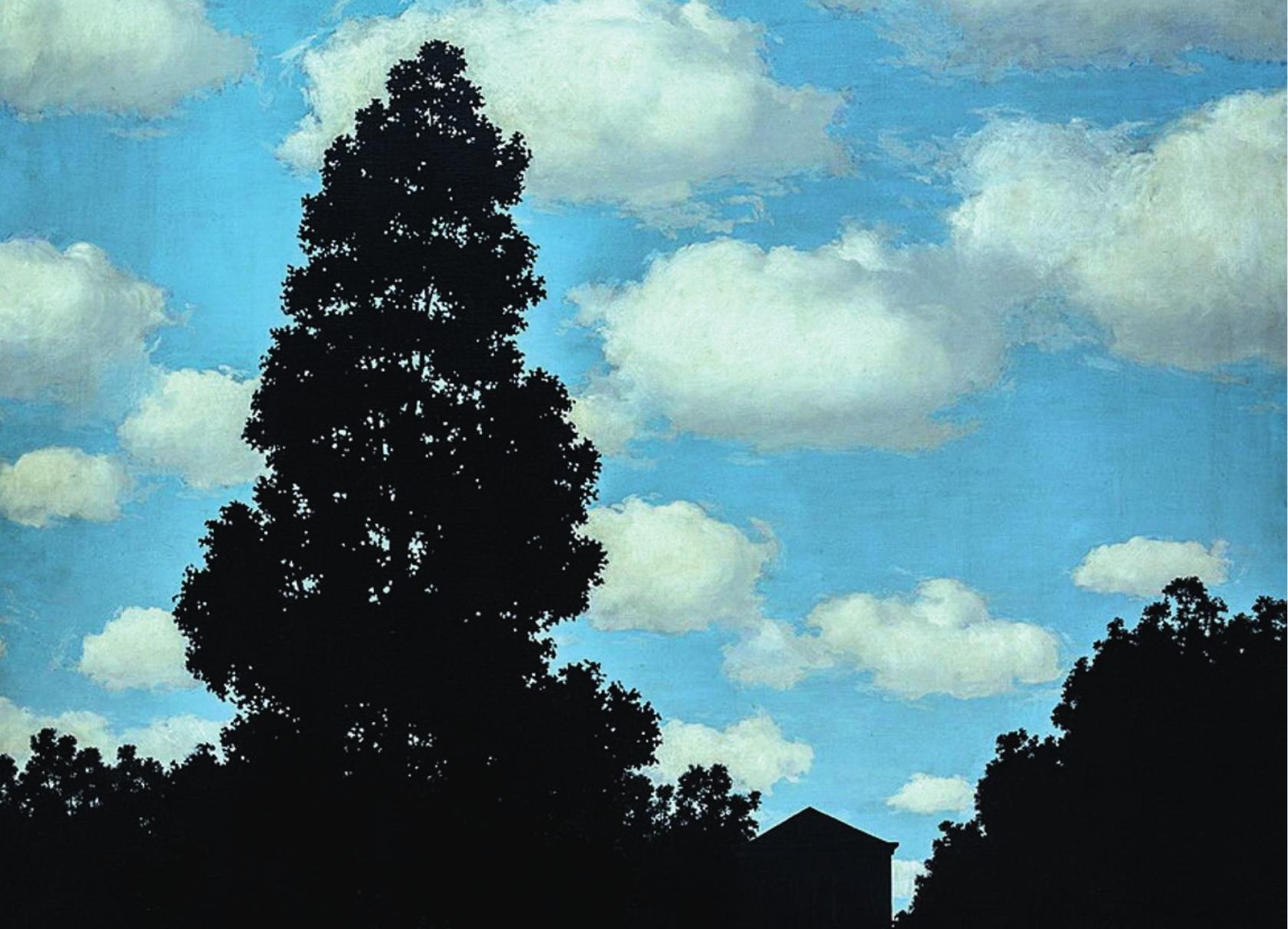

4t

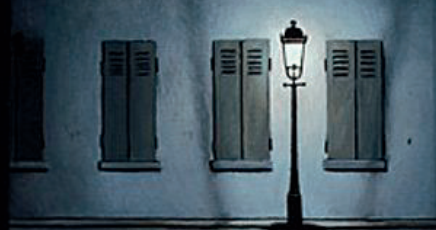


Appendices 


\section{NEDERLANDSE SAMENVATTING}

\section{Deel I}

Het eerste deel van dit proefschrift beschrijft de morfologische veranderingen die worden gezien in gezond hersenweefsel na bestraling voor gliomen. De reden dat we dit hebben onderzocht is het fenomeen van cognitieve achteruitgang bij patiënten die behandeld zijn voor hersentumoren. Naast het effect van de tumor zelf, en het effect van de operatie die veel patiënten ondergaan, wordt gedacht dat radiotherapie ook een rol speelt bij de achteruitgang van het cognitieve vermogen. Een van de mogelijke oorzaken is radiatiegeïnduceerde schade van gezond hersenweefsel, waardoor de normale functies van de hersenen verstoord worden. Omdat cognitie een complex proces is, waarbij verschillende hersengebieden samenwerken in een uitgebreid netwerk, hebben we gekeken wat het effect is van het geven van radiotherapie op elk deel van de hersenen. We hebben hiervoor MRI's verzameld van voor en na de bestraling, en de veranderingen in hersenmorfologie bepaald. Vervolgens hebben we deze veranderingen gerelateerd aan de dosis die plaatselijk op de hersenen is gegeven. Hiermee hebben we onderzocht of er een verband is tussen de gegeven dosis en de plaatselijke morfologische veranderingen.

In hoofdstuk 2 beschrijf ik het beschikbare bewijs dat radiotherapie geassocieerd is met morfologische veranderingen in de cerebrale cortex, voorafgaand aan ons eigen onderzoek. We hebben een systematische zoekopdracht gedaan om alle mogelijke artikelen te vinden, en hebben na een strenge selectieprocedure 21 artikelen gebruikt om tot een conclusie te komen. De kwaliteit van de artikelen was erg wisselend; er werd zowel longitudinaal gekeken naar veranderingen binnen dezelfde patiënten, als cross-sectioneel tussen bestraalde en niet-bestraalde groepen. Daarnaast waren er maar enkelen die ook het verband tussen de dosis en de mate van verandering hebben onderzocht. Uiteindelijk kwamen twee artikelen als beste naar voren, die beiden lieten zien dat er een verband is tussen hogere radiotherapiedosis en afname van de cortexdikte.

In hoofdstuk 3 onderzoeken we zelf het verschil in cortexdikte na bestraling, en of dit samenhangt met de gegeven dosis. We hebben hiervoor 28 patiënten geselecteerd die bestraald zijn voor een glioom, en waarvan MRI-scans beschikbaar waren van voor en I jaar na de behandeling. We hebben op geautomatiseerde wijze de lokale cortexdikte berekend met gespecialiseerde software. Door simpelweg de cortexdike voor en na de bestraling van elkaar af te trekken hebben we het verschil in cortexdikte berekend. Ten slotte hebben we dit verschil gerelateerd aan de lokale gegeven dosis, en zo berekend wat de associatie is tussen cortexdikte en bestralingsdosis. Er kwamen drie cortexgebieden naar voren, die elk een significant verband kenden tussen de dosis en het verschil in cortexdike. De afname van cortexdikte was in deze regio's 5 tot $26 \mu \mathrm{m} / \mathrm{Gy}$, wat overeenkomt met een 
verschil van 5.4 tot $21.6 \%$ per 30 Gy. Dit is vergelijkbaar met het verschil in cortexdikte als men tien jaar ouder wordt. Daarmee concluderen we dat het effect van radiotherapie op de cerebrale cortex aanzienlijk is, en dat dit een mogelijke verklaring kan zijn voor de cognitieve schade na bestraling.

In hoofdstuk 4 beschrijf ik een studie naar het effect van bestraling op de subcorticale kernen van het brein. Dit omdat niet alleen de hersenschors bijdraagt aan een normaal cognitief functioneren; de subcorticale kernen spelen hier ook een rol in. We hebben in 31 patiënten het effect van bestraling op de volgende structuren gemeten: amygdala, nucleus accumbens, nucleus caudatus, hippocampus, globus pallidus, putamen en thalamus. We vonden een verband tussen de gegeven dosis en volumeafname een jaar na bestraling voor al deze structuren, behalve de nucleus caudatus. Daarnaast hebben we de "hippocampusleeftijd" bepaald, afgeleid van de natuurlijke afname in hippocampusvolume met de leeftijd. We zagen dat, een jaar na bestraling, de mediane "hippocampusleeftijd" met II jaar toeneemt. Dit is dus een vergelijkbaar effect als gezien in de cortex, zoals beschreven in hoofdstuk 3. De subcorticale kernen zijn dus ook gevoelig voor dosisafhankelijke schade na bestraling.

In hoofdstuk $\mathbf{5}$ is het laatste dat gaat over het effect van bestraling op gezond hersenweefsel, en onderzoekt dit voor de gehele hersenen. In de voorgaande hoofdstukken werd slecht één specifiek deel van de hersenen bestudeerd, terwijl niet zeker is dat het verband tussen dosis en morfologie beperkt is tot een select aantal hersengebieden. Daarom is in dit hoofdstuk geen rekening gehouden met bepaalde functionele of anatomische structuren, maar is het brein in zijn geheel geanalyseerd. Dit is op drie manieren gedaan. Ten eerste werd gekeken naar het effect van bestraling op het totale breinvolume, en de volumina van de totale grijze en witte stof. Daarnaast werden twee recent ontwikkelde technieken gebruikt, voxel-based morphometry en deformation based morphometry. Dit zijn twee verschillende technieken om hetzelfde te meten, namelijk op millimeterniveau bepalen wat het effect van bestraling is op het volume van de hersenen, zonder dat daarbij onderscheid wordt gemaakt tussen witte stof, grijze stof, of liquor. Dit resulteerde in een significant verband tussen dosis en volume in verscheidene clusters in het brein, zowel in de witte als grijze stof. Daarnaast zagen we een afname van het totale breinvolume, met daarbij een compensatoire toename van het volume van de liquor. Ook dit is dus een duidelijk teken dat de gehele hersenen gevoelig zijn voor schade na bestraling.

\section{Deel II}

Het tweede deel van dit proefschrift beschrijft (lopend) onderzoek naar de overleving en de kwaliteit van leven van patiënten die bestraald zijn voor hersenmetastasen. Metastasen in de hersenen komen voor in 20 tot 40 procent van de patiënten met kanker; 
geschat wordt dat elk jaar bij zo'n 7,000 mensen in Nederland hersenmetastasen worden ontdekt. De meest voorkomende primaire tumorsoorten die ten grondslag liggen aan hersenmetastasen zijn longkanker, borstkanker en melanoom. De behandeling bestaat doorgaans uit (een combinatie van) systeemtherapie, neurochirurgie en bestraling. Deze laatste behandeling heeft de laatste tijd een ommezwaai gemaakt van het bestralen van de gehele herseninhoud (whole-brain radiotherapie) naar het specifiek bestralen van alleen de metastasen (stereotactische radiotherapie, ook wel radiochirurgie genoemd). Stereotactische radiotherapie zorgt voor een soortgelijke overleving, maar beperkt de dosis in gezond hersenweefsel, en gaat dus gepaard met minder bijwerkingen.

Hoofdstuk 6 beschrijft een onderzoek naar de uitkomsten van een veelgebruikt voorspelmodel voor de overleving van hersenmetastasepatiënten, en vergelijkt deze met de daadwerkelijke overleving. Dit model, de disease-specific Graded Prognostic Assessment (DS-GPA) schat overleving van patiënten met hersenmetastasen vanuit de vijf meest voorkomende primaire tumorlocaties: long, borst, melanoom, nier en darm. Op basis van enkele klinische factoren, zoals leeftijd, aantal hersenmetastasen, dagelijks functioneren en tumorspecifieke markers wordt met het model een score berekend, die past bij een geschatte mediane overleving. Deze wordt gebruikt om een beeld te schetsen van de uitkomsten, voor zowel de arts als de patiënt. We zagen dat op individuele basis de uitkomsten van de DS-GPA er flink naast kunnen zitten, tot wel 40 maanden. Op groepsniveau lag de schatting veel dichter bij de werkelijkheid; de werkelijke mediane overleving in een groep met een bepaalde DS-GPA score kwam overeen met de mediane overleving die bij die score past. Dit betekent dat men voorzichtig moet zijn met het interpreteren en uitleggen van de geschatte mediane overleving aan patiënten. Het is geen accurate voorspelling van de overleving na behandeling, het is een maat die zegt dat de helft van de patiënten met een soortgelijk tumorprofiel overlijdt binnen de aangegeven periode.

In hoofdstuk $\mathbf{7}$ laat ik zien welke factoren voorspellend zijn voor de overleving van patiënten met metastasen vanuit gynaecologische tumoren. Dit zijn is een zeer zeldzaam fenomeen, het komt voor in $0.3 \%$ tot $2.2 \%$ van de gevallen. De verwachting is wel dat, door betere behandelingen, patiënten met bijvoorbeeld een ovarium- of cervixcarcinoom een betere overleving hebben, en daarmee helaas meer tijd om hersenmetastasen te ontwikkelen. $\mathrm{Er}$ is nog niet veel bekend over welke factoren de overleving kunnen voorspellen, de hierboven genoemde DS-GPA bevat geen module voor gynaecologische tumoren. We hebben daarom gegevens verzameld van een groep van 73 patiënten met hersenmetastasen vanuit gynaecologische tumoren die behandeld zijn in twee centra in Utrecht en Boston, Verenigde Staten. We zagen dat leeftijd, primaire tumorsoort en de tumormarker CA-I25 voorspellend waren voor de overleving. Patiënten met een ovariumcarcinoom hadden een duidelijk gunstigere overleving, net als jongere vrouwen en diegene met een laag CA-125. 
Deze gegevens kunnen worden gebruikt om de overleving te schatten, en om grotere modellen zoals de DS-GPA te ontwikkelen.

Het effect van de sociaaleconomische status op overleving in hersenmetastasepatiënten wordt onderzocht in hoofdstuk 8. De sociaaleconomische status, of SES, is een manier om iemands inkomen, opleidingsniveau en arbeidspositie te meten. Idealiter is er natuurlijk geen verband tussen de SES en overleving, omdat in ons zorgsysteem iemands inkomen geen rol hoort te spelen in de toegang tot de zorg. In andere landen, ook in landen met verplichte zorgverzekeringen, wordt wel een verband gevonden tussen de SES en de overleving in patiënten met kanker. Daarom hebben we dit onderzocht in een groep hersenmetastasepatiënten, ook omdat gegevens over deze specifieke groep spaarzaam zijn. We vonden geen significant verband tussen de SES en overleving in onze groep patiënten, ook niet wanneer gecorrigeerd werd voor andere factoren. Het geschatte effect is daarnaast zodanig gering dat we kunnen concluderen dat er geen effect is op de overleving.

Het laatste hoofdstuk, hoofdstuk 9 , beschrijft de eerste resultaten van de COIMBRAstudie. In deze studie worden alle patiënten uitgenodigd die worden verwezen naar het UMCU voor bestraling van hersenmetastasen. De studie bestaat uit 4 onderdelen, waarvan er 3 optioneel zijn. Het enige vaste onderdeel is het verzamelen van gegevens, waarmee alle informatie vanuit het patiëntendossier wordt gebruikt om bijvoorbeeld de overleving te bestuderen. In het tweede onderdeel wordt deelnemers gevraagd om periodiek vragenlijsten in te vullen over de kwaliteit van leven. Hiervoor worden verschillende gevalideerde vragenlijsten gebruikt, die bijvoorbeeld de cognitie, vermoeidheid of depressie meten. Het derde onderdeel is het maken van extra MRI-scans aansluitend aan reeds geplande scans, om zo nieuwe soorten beeldvorming te testen. Het laatste onderdeel is de mogelijkheid om nieuw gerandomiseerd onderzoek te doen naar nieuwe behandelingen, volgende het zogenaamde trials within cohorts model. Hierin geven deelnemers van tevoren toestemming om gerandomiseerd te worden voor toekomstig onderzoek, waarbij nog niet bekend is welk onderzoek dit zal zijn. Zodra een onderzoek naar een nieuwe behandeling wordt gestart, en een patiënt wordt geloot om deze behandeling te ondergaan, wordt er opnieuw toestemming gevraagd voor deelname. Patiënten in de controlegroep horen in dit stadium niets, en weten dus niet dat zij als vergelijking dienen. Dit vergemakkelijkt het vinden van studiedeelnemers, en voorkomt teleurstelling in de groep patiënten die geen nieuwe veelbelovende behandeling ondergaan omdat zij in de controlegroep belanden.

Deze studie is gestart in 2019, en in dit hoofdstuk worden de resultaten na I jaar beschreven. Van de 153 benaderde patiënten wilde 73\% meedoen, waarbij de meerderheid koos voor de optionele onderdelen. De kwaliteit van leven op het moment van bestraling is vergelijkbaar met zowel kankerpatiënten in het algemeen als mensen met hersentumoren, wat aangeeft 
dat we een representatieve groep geïncludeerd hebben. Met het COIMBRA-onderzoek kunnen de komende jaren veel relevante klinische gegevens worden verzameld, en is er een manier om snel de nieuwste behandelingen te testen en valideren. Dit draagt bij aan de zorg voor patiënten met hersenmetastasen. 


\section{DANKWOORD}

Hoewel mijn naam de enige is die op de kaft van dit proefschrift staat, was deze er met zekerheid niet geweest zonder een flink aantal anderen. Hun toewijding, inzet, steun en/ of gezelligheid was nodig voor het tot stand komen van wat u nu in handen heeft. Daarom zou ik deze mensen graag hieronder willen bedanken.

Ten eerste zou ik mijn copromotoren willen bedanken: dr. J.J.C. Verhoeff en dr. T.J. Snijders. Beste Joost, erg bedankt voor de fijne begeleiding. Na iedere wekelijkse woensdagochtendsessie zat ik weer vol nieuwe ideeën, en jouw aanstekelijke enthousiasme zorgde ervoor dat ik die in de praktijk wilde brengen. Beste Tom, dankzij de wetenschapsstage die ik tijdens mijn geneeskundestudie onder jouw hoede heb gelopen ben ik de wetenschap leren waarderen, en je hebt me ook nog eens geholpen om een promotieplek te vinden. Bedankt voor de kritische noot op de wilde ideeën en teksten van Joost en mij, en nog de oprechte excuses dat ik uiteindelijk toch geen neuroloog ga worden.

Prof. dr. H.M. Verkooijen, beste Lenny, ik ben blij dat ik deel heb mogen uitmaken van je inmiddels kleine legertje van promovendi. Jouw inzet voor het testen en implementeren van nieuwe innovaties binnen de oncologische zorg is inspirerend, en jouw kennis van de epidemiologie is ongeëvenaard.

Leden van de beoordelingscommissie prof. dr. G.J. Biessels, prof. dr. T. Seute, prof. dr. P.C. de Witt Hamer, prof. dr. L.J.A. Stalpers, en prof. dr. M.J.E. van Zandvoort, hartelijk dank voor de tijd en aandacht die het beoordelen van dit proefschrift gekost heeft. Ik kijk er naar uit om met jullie van gedachten te wisselen over hetgeen jullie gelezen hebben.

Voor het schrijven van de hoofdstukken in dit proefschrift zijn natuurlijk mijn co-auteurs essentieel geweest, waarvan ik er een aantal in het bijzonder wil bedanken. Szabolcs, thank you for our extensive collaboration the last few years. Your tenacity in achieving reliable results is admirable, as well as your ability to be critical and realistic. Your radar for bullshit is unlike any other, and this thesis would be only half as long without your input. An, bedankt voor het mogen gebruiken van jouw hersenmetastasedatabase, en voor het meelezen en -schrijven aan de verschillende stukken die hieruit voortgekomen zijn. Alexander, bedankt voor de overzeese samenwerking en Marseillaanse gezelligheid.

Tijdens het opzetten en uitvoeren van het afstudeerproject van de postgraduate master Epidemiologie heb ik erg veel gehad de begeleiding van Sjoerd Elias. Bedankt voor de uitleg over de ingewikkelde analyses die nodig waren voor mijn onderzoek, en voor het kritisch bekijken van mijn ellelange R-code. 
Ik zou de leden van de APRICOT-studiegroep willen bedanken voor de samenwerking, en het meedenken en -schrijven aan de verschillende protocollen. Eva, we hebben het toch mooi voor elkaar gekregen om in redelijk korte tijd twee protocollen te schrijven en in te dienen, en ik ben blij om te horen dat de APRICOT nu een beetje loopt. Marielle, bedankt voor de hulp bij het indienen van de protocollen, en het meelezen met de verschillende artikelen. Fia, mijn COIMBRA-opvolger, bedankt dat je het stokje van me hebt overgenomen. Ik weet zeker dat ondanks onze wat warrige overdrachten na werktijd je er mooie en waardevolle dingen uit kunt halen.

Het doen van onderzoek is erg lastig zonder de medewerkers van het trialbureau van de divisie Beeld en Oncologie. In het bijzonder zou ik Saskia en Rosalie willen bedanken voor de hulp met het opzetten en draaiende houden van COIMBRA en APRICOT. Daarnaast zou ik ook iedereen van de cohortpoli, het secretariaat, de ICT, de stafleden, de AIOS, en alle andere Vrienden van de Radiotherapie willen bedankten voor alle ondersteuning.

Promoveren is natuurlijk hard werken, maar ook hard ontspannen is erg belangrijk. Daarom zou ik graag alle arts-onderzoekers van de radiotherapie willen bedanken voor jullie inzet om me vooral niet te veel te laten werken. Fieke, Lucas, Joris, Alicia, Jeanine, Sieske, Alice, Madelijn, Boris, Bart, Lois, Marieke, Marilot, Sophie, Anita, Ingmar, Veerle, Arthur, Thomas, Freek, Marcia, Hidde, Dieuwke, Floris, Maaike: wat hebben we geweldig geluncht, geborreld en gedanst. Bedankt voor alle leuke lunches, congressen, weekenden, en masterdagen. We komen elkaar vast nog een keer tegen, binnen of buiten het ziekenhuis.

Helaas heb ik mijn oude collega's vaarwel moeten zeggen, maar gelukkig heb ik er fijne nieuwe voor teruggekregen. Dorine, Nienke, Mandy, Guido, Jega, Claudia, Marloes, Marije, Rogier, en Janine: bedankt voor het fijne onthaal in Rotterdam, ik voel me al helemaal onderdeel van de gezellige en hechte AIOS-groep. Daarnaast zou ik ook de opleiders, Manouk en Marjan, en de overige stafleden van de radiotherapie in het Erasmus MC willen bedanken voor het warme welkom en de fijne begeleiding.

Als je drie jaar lang ergens werkt en (in het pre-covidium) elke dag met dezelfde mensen op een kamer moet zitten mag je hopen dat het een beetje leuke mensen zijn. Gelukkig was ik gezegend met ontzettend leuke kamergenoten van Q.0S.4.37. Hans, Sophie en Joris, onze kennismaking was aan de korte kant, maar desalniettemin was het toch een fijne samenwerking en hebben we fijne gesprekken gevoerd. Bedankt nog voor het wegwijs maken op mijn eerste werkdag, midden in de kerstvakantie. Soraya, bedankt voor de lessen Italiaans (ik weet voor de rest mijn leven hoe ik in Italië met mijn pinpas moet betalen), en een onvergetelijke Aperol Spritz. Van al onze promotieonderwerpen was die 
van jou het meest ondoorgrondelijk, erg knap hoe je hier chocola van hebt kunnen maken, en je tegelijkertijd allerlei rare Nederlandse spreekwoorden eigen hebt gemaakt. Dennis, jij hebt de indrukwekkende vaardigheid om hard te werken maar er eigenlijk nooit te zijn. Op de zeldzame momenten dat je ons verblijdde met je aanwezigheid hebben we veel van je kunnen leren, met name over de edele kunst van het afdingen. Daan, iemand die zowel het orgel als Matlab uitstekend kan bespelen. Het is fijn om iemand te kennen die op een geweldige manier zowel frustraties als fascinaties kan ventileren, en die zowel zijn promotie als de rest van zijn leven op een indrukwekkende manier op de rails heeft. Prescilla, het was erg leuk dat je (met enige dwang wellicht) op onze kamer bent komen zitten, en om je verhalen over Harm aan te horen. Mick, poulemaster en (oud) mede-Sypbewoner, bedankt voor de nodige afleiding. Ik had nooit gedacht dat ik zo geïnteresseerd kon raken in datende boeren of BN'ers die op exotische locaties stomme spelletjes spelen, en ik zal het bruggetje in Fiji ook nooit vergeten. Thomas, aanstaand collega en uitstekend borrelgezelschap, erg leuk dat je binnenkort ook aan de opleiding gaat beginnen, en dat we elkaar ook na mijn promotietijd nog regelmatig zien. Jorine, bedankt voor onze wilde avonturen naar dierentuinen, pretparken, dansavonden en huishoudbeurzen, en ontzettend leuk dat jij mijn paranimf wil zijn.

En dan mijn oudste vriendengroep, aka de Boys. We kennen elkaar inmiddels al meer dan vijftien jaar, en nog altijd wonen we vlak bij elkaar en zien we elkaar regelmatig. Het is erg spijtig dat ik nu van plan ben om zo ver weg te verhuizen, maar ik weet zeker dat we nog vaak zullen afspreken. Bedankt voor alle leuke avonden de afgelopen jaren, als ik bij jullie ben voel ik me weer helemaal jong. Jeroen, jou ken ik waarschijnlijk het langst van allemaal. We hebben samen ontzettend veel concerten bezocht, en ik ben blij dat we er alweer een paar in de planning hebben staan. Aga, mijn running mate. We zijn tegelijk aan onze promotie begonnen, en ondanks dat de onderwerpen ver uiteenliepen heb ik veel aan je wijsheden gehad. Ik weet zeker dat je een ontzettend goede cardioloog gaat worden, en vind het geweldig dat jij mijn paranimf bent. Imre, oud D2West-collega en mede-kringloopfanaat, nog altijd sturen we elkaar de raarste grappen en feitjes door, en geven we elkaar ongevraagd stijladvies. Erg leuk dat we een paar maanden samen op zaal hebben gewerkt, en jij me hebt kunnen overrompelen met je elektrolytenkennis. Als de cijfers rood zijn weet ik bij wie ik moet zijn. Jorrit, onze enige niet-medicus, ik ben zeer onder de indruk van jouw status als zakenman, innovator en vrije geest. Het is erg mooi om te zien hoe jij je bedrijf hebt opgebouwd, en hoeveel je weet over technologische vooruitgangen. Jouw doortastendheid is als geen ander, jij komt er wel.

Als laatste zou ik mijn familie willen bedanken. Mijn zus Sarah, Saar, we hebben altijd goed op kunnen schieten, zowel toen we nog jong waren als nu. Het is fijn om iemand in mijn leven te hebben die net zo enthousiast is over leuke weetjes, fijne hitjes en het 
Eurovisiesongfestival. Als ik iets nieuws te vertellen heb, iets raars heb meegemaakt, of gewoon een scherpe observatie, frustratie, of flauw grapje met iemand wil delen, ben jij de eerste aan wie ik denk. Mijn ouders, Nico en Hetty, het is niet mogelijk om op te schrijven wat ik allemaal aan jullie te danken heb. In ieder geval mijn interesse in muziek en kunst heb ik thuis aangeleerd gekregen, net als mijn manier van denken en altijd meer over dingen willen weten. Nog altijd kom ik regelmatig langs op zondag, om bij te praten, kunst te bekijken en vooral heel veel thee te drinken. Jullie hebben heel veel mogelijk gemaakt. Zonder jullie was ik nergens. 


\section{LIST OF PUBLICATIONS}

I. EE van Grinsven, SHJ Nagtegaal, JJC Verhoeff, MJE van Zandvoort. The Impact of Stereotactic or Whole Brain Radiotherapy on Neurocognitive Functioning in Adult Patients with Brain Metastases: A Systematic Review and Meta-Analysis. Oncol Res Treat. 2021;44(II):622-636

2. SHJ Nagtegaal, S David, EE van Grinsven, MJE van Zandvoort, E Seravalli, TJ Snijders, MEP Philippens, JJC Verhoeff. Morphological changes after cranial fractionated photon radiotherapy: localized loss of white matter and grey matter volume with increasing dose. Clin Transl Radiat Oncol. 202I Aug 29;31:14-20

3. SHJ Nagtegaal, S David, MEP Philippens, TJ Snijders, A Leemans, JJC Verhoeff. Dose-dependent volume loss in subcortical deep grey matter structures after cranial radiotherapy. Clin Transl Radiat Oncol. 2020 Nov 15;26:35-41

4. SHJ Nagtegaal, AFC Hulsbergen, EBL van Dorst, VK Kavouridis, CAC Jessurun, MLD Broekman, TR Smith, JJC Verhoeff. Age, pathology and CA-125 are prognostic factors for survival in patients with brain metastases from gynaecological tumours. Clin Transl Radiat Oncol. 2020 May 6;24:1 I- I5

5. SHJ Nagtegaal, S David, TJ Snijders, MEP Philippens, A Leemans, JJC Verhoeff. Effect of radiation therapy on cerebral cortical thickness in glioma patients: treatment-induced thinning of the healthy cortex. Neurooncol Adv. 2020 May 21;2(I):vdaa060

6. SHJ Nagtegaal, ALHMW van Lier, AA den Boer, MCA Kramer, G Fanetti, WSC Eppinga, MEP Philippens, JJC Verhoeff, E Seravalli. Does an immobilization mask have added value during planning magnetic resonance imaging for stereotactic radiotherapy of brain tumours? Phys Imaging Radiat Oncol. 2020 Mar 6;13:7-13

7. SHJ Nagtegaal, A Claes, KPM Suijkerbuijk, FMNH Schramel, TJ Snijders, JJC Verhoeff. Comparing survival predicted by the diagnosis-specific Graded Prognostic Assessment (DS-GPA) to actual survival in patients with $1-10$ brain metastases treated with stereotactic radiosurgery. Radiother Oncol. 2019 Sep;138:173-179

8. $\quad$ AFC Hulsbergen, M Mammi, SHJ Nagtegaal, AM Lak, V Kavouridis, TR Smith, JB lorgulescu, RA Mekary, JJC Verhoeff, MLD Broekman, JG Phillips. Significance of Programmed Death Receptor Ligand One Expression in Brain Metastases of Non-Small Cell Lung Carcinoma. Int J Radiat Oncol Biol Phys. 2020 Sep I;108(I):258-267

9. SHJ Nagtegaal, S David, ATJ van der Boog, A Leemans, JJC Verhoeff. Changes in cortical thickness and volume after cranial radiation treatment: A systematic review. Radiother Oncol. 2019 Jun; 135:3342 


\section{CURRICULUM VITAE AUCTORIS}

Steven Nagtegaal was born on the $5^{\text {th }}$ of February 1993 in Nieuwegein, and grew up there with his sister and parents. After graduating from Anna van Rijn College in Nieuwegein in 20II, he started Medical School in Utrecht.

During his last year of study, Steven started a research internship at the department of Neurology of the University Medical Center Utrecht under supervision of dr. T.J. Snijders. After graduating Medical School in 2017, he continued his research career at the department of Radiotherapy of the University Medical Center Utrecht with a three-year PhD project. He was supervised by prof. dr. H.M. Verkooijen, dr. J.J.C. Verhoeff and dr. T.J. Snijders. During this time, he initiated the COIMBRA-trial, a observational cohort into quality of life of patients with brain metastases, which can be used as a basis for conducting multiple randomized controlled trials. Additionally, he studied the effects of radiotherapy dose on healthy brain tissue in patients with glioma by analyzing MR imaging. In 2018, he started the postgraduate master Epidemiology at Utrecht University, completing both the Clinical Epidemiology and Medical Statistics specializations.

After finishing his PhD project, he started working as a resident not in training (ANIOS) at the department of Internal Medicine, where he worked for six months. In July 202I, he started as a resident in training (AIOS) at the department of Radiotherapy of the Erasmus Medical Center in Rotterdam, with the goal to continue working on research projects to further improve clinical care for patients with cancer. 